\title{
Die Matrikel der Wiener \\ Rechtswissenschaftlichen Fakultät
}

\section{Matricula Facultatis Juristarum Studii Wiennensis}

\author{
Im Auftrag der Universität Wien \\ herausgegeben von Kurt Mühlberger, \\ Archiv der Universität Wien \\ I. Band $1402-1442$ \\ Bearbeitet von Johannes Seidl \\ unter Mitarbeit von Andreas Bracher \\ und Thomas Maisel
}

Oldenbourg Böhlau 
Die Matrikel der Wiener

Rechtswissenschaftlichen Fakultät 


\author{
Publikationen des Instituts \\ für Österreichische Geschichtsforschung
}

\author{
VI. Reihe \\ Quellen zur Geschichte der Universität Wien
}

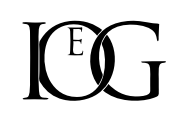

3. Abteilung

\author{
Die Matrikel der Wiener \\ Rechtswissenschaftlichen Fakultät
}

2011

Böhlau Verlag Wien

Oldenbourg Verlag München 


\title{
Die Matrikel der Wiener Rechtswissenschaftlichen Fakultät
}

\section{Matricula Facultatis Juristarum Studii Wiennensis}

\author{
Im Auftrag der Universität Wien
}

\author{
herausgegeben von \\ Kurt Mühlberger \\ Archiv der Universität Wien
}

I. Band: 1402-1442

Bearbeitet von

Johannes Seidl

unter Mitarbeit von

Andreas Bracher und Thomas Maisel

2011

Böhlau Verlag Wien

Oldenbourg Verlag München 


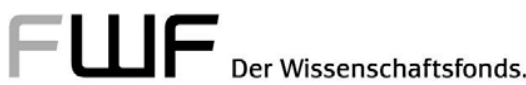

Gedruckt mit Unterstützung durch den Fonds zu Förderung der wissenschaftlichen Forschung

Bibliografische Information der Deutschen Nationalbibliothek:

Die Deutsche Nationalbibliothek verzeichnet diese Publikation in der

Deutschen Nationalbibliografie;

detaillierte bibliografische Daten sind im Internet über http://dnb.d-nb.de abrufbar.

\section{ISBN 978-3-205-78656-6 (Böhlau Verlag) \\ ISBN 978-3-486-70460-0 (Oldenbourg)}

Das Werk ist urheberrechtlich geschützt. Die dadurch begründeten Rechte, insbesondere die der Übersetzung, des Nachdruckes, der Entnahme von Abbildungen, der Funksendung, der Wiedergabe auf fotomechanischem oder ähnlichem Wege, der Wiedergabe im Internet und der Speicherung in Datenverarbeitungsanlagen, bleiben, auch bei nur auszugsweiser Verwertung, vorbehalten.

(C) 2011 by Böhlau Verlag Ges.m.b.H. und Co.KG, Wien, Köln, Weimar

http://www.boehlau.at

http ://www.boehlau.de

Gedruckt auf umweltfreundlichem, chlor- und säurefrei gebleichtem Papier.

Gesamtherstellung : Wissenschaftlicher Bücherdienst, Köln 


\section{INHALT}

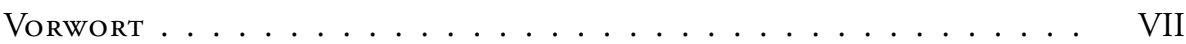

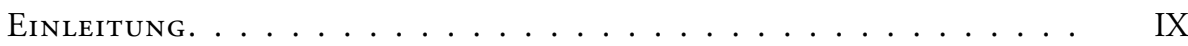

I. Einrichtung der Matrikel . . . . . . . . . . . . . . . . . . IX

II. Beschreibung der Vorlage . . . . . . . . . . . . . . . X X

1. Überlieferung und äußerer Zustand . . . . . . . . . . . . . . . . . X X

2. Beschreibstoff und Größe . . . . . . . . . . . . . . . . . . . XI X X

3. Blattzählung . . . . . . . . . . . . . . . . XI

4. Lagenordnung . . . . . . . . . . . . . . . . X XI

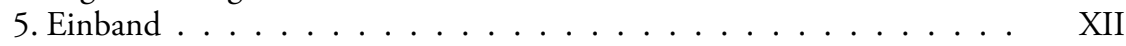

6. Schrift, Schreiber und Matrikelführung . . . . . . . . . . . . . . . XII

III. Statistik ..................... . XIV

1. Intitulationen und Promotionen . . . . . . . . . . . . XIV

2. Ständische Gliederung und Taxen . . . . . . . . . . . . . . XVI

3. Regionale Herkunft der Intitulierten . . . . . . . . . . . . . . XVII

IV. Grundsätze der Edition . . . . . . . . . . . . . . . XIX

V. Die Dekane .................... . . . XX

VI. Abkürzungen und Hilfsmittel . . . . . . . . . . . . . . . . . . . XXII

1. Kurzzitate (Quellen und Literatur) . . . . . . . . . . . . . . . . XXII

2. Verzeichnis der verwendeten Ortsnamenbücher und Karten . . . . . . . XXIV

3. Abkürzungen im Text und in den Registern . . . . . . . . . . . XXV

Text der Matrikel $1402-1442 \ldots \ldots \ldots \ldots$

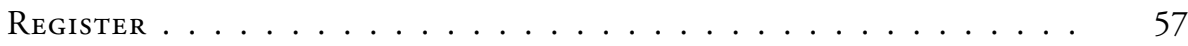

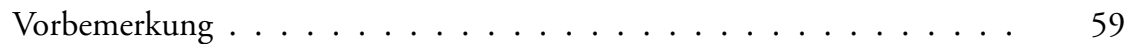

Register der Vornamen . . . . . . . . . . . . . . . . . . . . 61

Register der $\mathrm{Zu}$ - und Ortsnamen . . . . . . . . . . . . . . 103 



\section{VORWORT}

Das erste Matrikelbuch der Wiener Juristen wurde im Jahr 1402 aufgrund eines Beschlusses der Universitätsversammlung eingeführt und reicht bis zum Jahr 1442. Es handelt sich um die älteste Quelle zur Geschichte der gelehrten Universitätsjuristen im deutschen Sprachraum. Der Kodex stellt ein erstrangiges Denkmal für die Erforschung der Personen- und Institutionengeschichte der Juridischen Fakultät sowie zur Sozialgeschichte der spätmittelalterlichen Gelehrtenwelt dar.

Die Eintragungen der Dekane in das „Album“ der Juristen zeigen nicht nur den Eintritt in die Fakultät, oft wurde auch die erfolgte Graduierung festgehalten, sodass in diesen Fällen die Dauer des Studiums zu ermitteln ist. Darüber hinaus finden wir Angaben zur regionalen und sozialen Herkunft der Universitätsbesucher sowie die jeweilige Höhe der sozial gestaffelten Matrikeltaxen. Dem Editionstext ist eine Beschreibung der Originalhandschrift und eine Liste der amtierenden Dekane beigefügt. Die Matrikeledition wird durch ein Register der Vornamen sowie der $\mathrm{Zu}$ - und Ortsnamen erschlossen, das mit Hinweisen auf die zeitgenössischen, gedruckten Quellen der Universität erweitert wurde.

An der Entstehung dieser Edition waren mehrere Personen sehr hilfreich beteiligt, denen großer Dank gebührt. In erster Linie sind es die Bearbeiter, denen die Erstellung des Editionstextes samt Apparat, der Register und die einleitende Besprechung der Quelle oblag: Den Löwenanteil dieser Arbeit besorgte Doz. Mag. Dr. Johannes Seidl, aufbauend auf Vorarbeiten von Dr. Andreas Bracher, der den ersten Rohtext erstellt hatte, mit tatkräftiger redaktioneller Mitwirkung von Mag. Thomas Maisel. Für die Identifizierung zahlreicher Adelsnamen sei Herrn Univ.-Prof. Dr. Christian Lackner, Institut für Österreichische Geschichtsforschung, herzlich gedankt. In gleicher Weise gebührt Herrn Mag. Dr. Christian Domenig vom Historischen Institut der Universität Klagenfurt sowie Frau Mag. Martha Jauernig von der Österreichischen Historischen Bibliographie für wertvolle Hilfestellungen bei der korrekten Darstellung slowenischer Ortsnamen großer Dank. Ganz besonders sei Herrn em. Univ.-Prof. Dr. Winfried Stelzer, Institut für Österreichische Geschichtsforschung, für die fachkundige Begleitung dieses Projektes, zahlreiche wertvolle Ratschläge sowie für die Durchsicht des Editionstextes und der Einleitung gedankt.

Schließlich danke ich dem Böhlau Verlag, namentlich Frau Dr. Ursula Huber und Herrn Michael Rauscher, für die sachkundige Betreuung der Herstellung. Den Druck ermöglichte durch die finanzielle Unterstützung dankenswerter Weise der Fonds zur Förderung der wissenschaftlichen Forschung.

Kurt Mühlberger 



\section{EINLEITUNG}

\section{EINRIChtUng DeR Matrikel}

Der vorliegende Editionsband der Wiener Juristenmatrikel umfasst den ältesten Originalband der „Matricula Facultatis Juristarum Studii Wiennensis“ (MFJ I), der sich im Archiv der Universität Wien unter der Signatur J 1 befindet. Die Matrikelführung setzt gegen Ende des Wintersemesters 1401/02 ein. Die älteste Eintragung bezieht sich auf eine Universitätsversammlung vom 8. März 1402, in welcher aufgrund eines Antrages der Juristenfakultät aus dem Vorjahr ${ }^{1}$ der Beschluss gefasst wurde, dass neu eintretende Studenten der Rechte nunmehr dazu verpflichtet wären, sich nach der Einschreibung in die Hauptmatrikel der Universität (durch den Rektor) auch in die Matrikel der Fakultät gegen Aufnahmetaxen (vom Dekan) eintragen zu lassen. Der vorliegende Matrikelband verdankt wohl diesem Universitätsbeschluss seine Entstehung. ${ }^{2}$

Nach den ältesten Statuten der Juridischen Fakultät vom 1. April 1389 war eine gesonderte Matrikelführung durch den Dekan der Juristen nicht vorgesehen, sodass man davon ausgehen kann, dass es vor diesem Band keinen älteren Vorgänger gegeben hat. ${ }^{3}$ Für Studenten und Absolventen der Rechtsfakultät war ursprünglich bloß die Einschreibung in die Universitätsmatrikel verpflichtend gewesen. Vor der Promotion eines Kandidaten der Rechte war dieser Umstand vom Dekan zu überprüfen. Mit der Neueinführung der Juristenmatrikel im Jahre 1402 wurden gleichzeitig die entsprechenden Matrikeltaxen festgelegt. ${ }^{4}$

Für die Übernahme des Amtes des Dekans der Rechtswissenschaftlichen Fakultät war keine Wahl vorgesehen. Entscheidend war hingegen das Seniorat. Nach den Bestimmungen der Fakultätsstatuten sollte die Dekanswürde in der Reihenfolge des „Dienstalters“ vom ältesten der Fakultät angehörenden Doktor bis zum jüngsten, danach vom ältesten Lizentiaten bis zum jüngsten, schließlich wieder bei dem ältesten Doktor beginnend weitergegeben werden. Nach der Wahl des Rektors, welche den Prokuratoren der vier Aka-

1 Die Antragstellung der Fakultät an die Universitätsversammlung wurde auf dem Spiegelblatt des vorderen Buchdeckels des Matrikelbandes mit Verweis auf die Acta Universitatis festgehalten: Folio XX $X^{\circ}$ in rectoratu magistri Johannis Berwart de Villingen. Anno domini $M^{\circ} C C C C^{\circ}$ primo. Item decanus facultatis juristarum nomine facultatis eiusdem supplicat, quod universitas dignaretur approbare et eidem facultati concedere, [...] Et placuit universitati supplicatio et admittebatur illo tamen adiecto, quod illa facultas nullum matricule sue inscribet, nisi talis prius per rectorem universitatis sit intitulatus et registra eiusdem inscriptus. Vgl. Anm. 2.

2 Zur Beschlussfassung über die Einführung der Juristenmatrikel vgl. Acta Universitatis I/2, UAW, Cod. R 1.2, fol. 20v, Uiblein, AFA I, 207 Anm. 26 und im Editionstext der Matrikel fol. $2^{\mathrm{r}}$ : Item statuimus, quod quilibet volens intrare de novo scolas et studere in iure, postquam intitulatus fuerit in matricula universitatis, debet etiam intitulari in matricula facultatis iuris per ipsius decanum. [...].

3 Die Fakultätsstatuten s. bei KINK 2, 139 und 145.

4 Siehe dazu im Abschnitt „Ständische Gliederung und Taxen“, Seite XVI. 
demischen Nationen vorbehalten war, ${ }^{5}$ sollte das Amt des Dekans demnach automatisch auf den jüngeren Doktor oder Lizentiaten übergehen. ${ }^{6}$ Diese Reihenfolge wurde in der Realität nicht sehr genau eingehalten. Es wechselten Doktoren und Lizentiaten in bunter Folge.

Folgt man dem Wortlaut der Statuten, so kann man die Termine der Rektorswahlen für die zeitliche Einordnung der semesterweisen Eintragungen der Juristendekane heranziehen. Die Wahl des Rektors erfolgte üblicherweise am 14. April (festum S. Tiburtii et Valeriani) für das Sommersemester (in der Edition und im Register mit „I“ bezeichnet, z. B. „1402 I“) und am 13. Oktober (festum S. Colomanni) für das Wintersemester („II“). In manchen Jahren ist man von diesem Brauch jedoch um einige Tage abgewichen. ${ }^{7}$

\section{Beschreibung Der Vorlage}

\section{1. Überlieferung und äußerer Zustand}

Der Pergamentkodex (Cod. J 1) wurde von Dekan zu Dekan weitergereicht und ist offenbar dem Archiv der Juridischen Fakultät spätestens unter dem Fakultätsarchivar Anton Hye von Gluneck einverleibt worden. Dieser übernahm 1836 auch das Amt des Universitätsarchivars und führte die Registraturen und Archive der Teilkorporationen im Universitätsarchiv zusammen. In einem Handschriftenverzeichnis seines Nachfolgers Karl Schrauf scheint der Band unter einer Reihe von Manuskripten auf, die im Zeitraum 1873-1880 dem Archiv der Universität Wien übergeben wurden. ${ }^{8}$ Eine zeitweise Entfremdung des Bandes ist in diesem Falle nicht festzustellen. Bezüglich Anschaffung und Anlegung des Bandes sind keine direkten Hinweise bekannt. Auf Blatt 1 recto befindet sich ein Rundstempel der Universität Wien aus dem 19. Jahrhundert mit der Umschrift „C. R. UNIVERS: VIENNEN:“, der das Wappen der Universität Wien zeigt. Dieser

5 Die erste Amtshandlung der von den Akademischen Nationen gewählten Prokuratoren war die Wahl des Rektors. S. dazu Die Matrikel der Ungarischen Nation an der Wiener Universität 1453-1630, ed. Karl Schrauf (Wien 1902), s. Einleitung, S. XIX-XXII.

6 Wir finden daher in den einleitenden Texten zu den einzelnen Semestern fast durchwegs die Formulierung [...] in decanum facultatis iuris (canonici) assumptus est [...], die auf die automatische Amtsübernahme hindeutet. Vgl. z.B. 1408 I, 1408 II, 1409 I etc. Zur Amtsübernahme des Juristendekans siehe den Abschnitt De decano der Fakultätsstatuten vom 1. April 1389 bei KINK 2, 137-141, bes. S. $138 f$.

7 Während der Funktionsperioden von 1401 II bis 1442 I war das in folgenden Semestern der Fall: 1405 I (11. April, vigilia Palmarum), 1408 I (9. April, die nona Aprilis, que tunc erat feria secunda ante festum Tiburcii et Valeriani. Man wollte den Sonntag vermeiden, auf Montag den 15. April fiel in diesem Jahr das Osterfest, daher wurde die Rektorswahl eine Woche vorverlegt), 1435 I (c. 14. April, circa festum beatorum Tiburcii et Valeriani), 1436 II (25. November, in die beate Katherine virginis et martiris. Grund war eine Pestepidemie). In einigen Semestern ist der Termin der Rektorswahl in der Hauptmatrikel nicht ausdrücklich angegeben: 1402 II, 1412 II, 1420 II. Vgl. die einleitenden Texte zu den einzelnen Semestern in GaLl, MUW I, 1377-1450 (1956) und Cod. NR 1 fol. 57va 60v (zu den Semestern 1435 I und 1436 II). Das Wahldatum ist in diesen Fällen jedoch über die Acta Universitatis bzw. über die Matrikel der Rheinischen Nation zu erschließen: 1402 II (11. April 1405 nach AU I/2, Cod. R 1.2, fol. $24^{v}$ ), 1412 II (13. Oktober 1412 nach AU II, Cod. R 1.2, fol. 51 ) und 1420 II (13. Oktober 1420 nach AU II, Cod. R.1.2, fol. $110^{\mathrm{r}}$ ).

8 „Interimistisches Verzeichnis der seit 1873 (bis 1880) dem Universitäts-Archive übergebenen Manuscripte etc.“, UAW, Akademischer Senat, GZ 8 aus 1880/81; Juridische Fakultät, Fasz. I, GZ 7173, 7176, 7410 aus 1833/34. Vgl. Kurt MüHlberger, Marija Wakounig, Vom Konsistorialarchiv zum Zentralarchiv der Universität Wien. Die Neuorganisation und Erweiterung des Archivs der Universität Wien im 19. Jahrhundert unter der Einflußnahme Theodor von Sickels. In: Scrinium 35 (Wien 1986), S. 190-213. 
Stempel dürfte bei der Übertragung des Bandes vom Archiv der Juridischen Fakultät in das Universitätsarchiv als Eigentumsvermerk angebracht worden sein.

Der Kodex weist mehrfach jüngere Restaurierungsspuren (Erneuerung des Buchrückens, Ausbesserungen am Einband) auf, die letzte Restaurierung erfolgte im Jahre 2003, wobei die beiden in Verlust geratenen Buckeln ergänzt wurden. ${ }^{9}$

\section{Beschreibstoff und Größse}

Es handelt sich um einen gut erhaltenen Pergamentkodex, die Maße des Buchblockes betragen $23 \times 17,5 \mathrm{~cm}$ (fol. 1 ist um $1 \mathrm{~cm}$ schmäler). Der Schriftspiegel bewegt sich zwischen 18-20 cm (Höhe) und 12-13 cm (Breite). Die Namen wurden zunächst in Kolumnen eingetragen, ab Blatt 5 (1410 II) wurde der Schriftspiegel, dessen Ränder oft mit Bleistift oder Tinte vorgezeichnet sind, in durchgehenden Zeilen beschrieben.

\section{Blattzählung}

Der Band besitzt eine Blattzählung, die nach Abschluß der Eintragungen in Tintenschrift in einem Arbeitsgang jeweils am rechten oberen Rand eingefügt wurde. Die Foliierung wurde vom zweiten Blatt der ersten Lage bis zum letzten (am hinteren Buchdeckel aufgeklebten) Blatt der letzten Lage ohne Zählung des fehlenden Blattes vorgenommen. Es wurden insgesamt 36 Folien gezählt. Ein Hinweis auf diese Foliierung ist auf Blatt 1 recto von einer Hand des 19. Jahrhunderts mit Bleistift vermerkt („fol. 1-36“). Die Tintenfoliierung auf Blatt 2, auf dem der Haupttext der Matrikel mit der Passage In nomine Domini. Amen. Matricula facultatis iuristarum studii Wiennensis beginnt, wurde nachträglich auf „2“ (zuerst als „1“ bezeichnet) korrigiert, die übrige bestehende Foliierung jedoch durchgehend belassen. Offenbar wollte man zuerst die Zählung mit dem Einsetzen des Haupttextes beginnen, hat diese Idee aber dann wieder verworfen. Diese Korrektur wurde bei der Edition nicht berücksichtigt. Das fehlende - möglicherweise herausgeschnittene - Blatt nach folio 34 war offenbar bereits zum Zeitpunkt der Foliierung nicht vorhanden.

\section{Lagenordnung}

Die Pergamenthandschrift besteht aus vier Lagen im Umfang von 36 Folien. Es handelt sich um Quinionen (jeweils fünf Doppelblätter), deren erstes Blatt vorne und das letzte Blatt hinten jeweils als Spiegelblatt auf den inneren Bucheinband geklebt und mittels Pergamentohr um die erste bzw. letzte Heftlage gefaltet wurde. Die beiden Pergamentspiegel sind somit Teil der ersten bzw. vierten Lage. Bei der Foliierung wurde das erste Spiegelblatt nicht, das letzte hingegen schon berücksichtigt. Daraus ergibt sich die Differenz zwischen Blatt- und Lagenzählung. ${ }^{10}$

9 UAW, Restaurierungsprotokoll zu Cod. J 01, Jänner 2003 der Firma Somers-Punz, Buch- und Papierrestaurierung, St. Leonhard am Forst, mit Bilddokumentation.

10 Zur Beschreibung der Lagen vgl. Otto Mazal, Lehrbuch der Handschriftenkunde (= Elemente des Buchund Schriftwesens, Band 10, Wiesbaden 1986) S. 68f. - Nach der Chroust'schen Lagenformel ergibt sich: $(\mathrm{V}-1)^{8}+\mathrm{V}^{18}+\mathrm{V}^{28}+(\mathrm{V} 1)^{36}$. 
Die Anordnung der Lagen zeigt folgendes Bild:

\begin{tabular}{|c|c|c|c|}
\hline Lage I & Lage II & Lage III & Lage IV \\
\hline $04+05$ & $13+14$ & $23+24$ & $32+33$ \\
\hline $03+06$ & $12+15$ & $22+25$ & $31+34$ \\
\hline $02+07$ & $11+16$ & $21+26$ & $30+8$ \\
\hline $01+08$ & $10+17$ & $20+27$ & $29+35$ \\
\hline $00+, \mathrm{Ohr}^{\text {“ }}$ & $09+18$ & $19+28$ & „Ohr“ +36 \\
\hline
\end{tabular}

\title{
5. Einband
}

Der Einband ( $24 \times 18 \times 2,4 \mathrm{~cm}$ ) besteht aus mit hellbraunem Ziegenleder überzogenen Buchenholzdeckeln. Vorderer und hinterer Buchdeckel sind mit je fünf Messingbuckeln versehen, von denen jeweils einer in Verlust geraten ist. Buchdeckel und Buchrücken weisen starke Gebrauchsspuren auf und wurden in jüngerer Zeit teilweise ausgebessert. Anläßlich einer Restaurierung wurde der Buchrücken teilweise mit braunem Leder ergänzt, darauf in Goldprägung die Beschriftung „MATR. FAC. JUR. I 1402-1442“ angebracht. Die Buchdeckeln wurden mit zwei intakten Metallschließen und einem Lederband fixiert. Auf der Außenseite ist die weitgehend verblasste Aufschrift in gotischer Textualis erkennbar:

\section{[...] Matricula $1^{a}$ ab [anno] 1402 usq[ue] 1442}

Auf die Innenseite des vorderen Buchdeckels wurde das erste Blatt der ersten Lage als Spiegelblatt aufgeklebt und mittels Pergamentohr in die Heftung einbezogen. Ebenso wurde das letzte Blatt der letzten Lage auf die Innenseite des letzten Buchdeckels ganzfl̈chig aufkaschiert und mittels Pergamentohr mit der Heftung verbunden.

Der nachstehende Text, der auf die Einführung der Matrikel und auf die einzuhebenden Taxen Bezug nimmt, befindet sich auf dem vorderen Spiegelblatt der Handschrift: ${ }^{11}$

\begin{abstract}
Folio $X X^{\circ}$ in rectoratu magistri Johannis Berwart de Villingen. Anno domini $M^{\circ} C C C C^{\circ}$ primo. Item decanus facultatis juristarum nomine facultatis eiusdem supplicat, quod universitas dignaretur approbare et eidem facultati concedere, primo quod quilibet scolaris de novo intrans scolas juristarum teneatur dare decano duos grossos et baccalarius de novo intrans tres et nobilis vel statum nobilium tenere volens medium florenum. Et qui in baccalarium promovetur 1 florenum, licenciatus vero tres florenos Ungaricales. Et placuit universitati supplicatio et admittebatur illo tamen adiecto, quod illa facultas nullum matricule sue inscriberet, nisi talis prius per rectorem universitatis sit intitulatus et registra eiusdem inscriptus.
\end{abstract}

\section{Schrift, Schreiber und Matrikelführung}

Der Band ist durchwegs in gotischer Geschäftsschrift (sorgfältige Bastarda) im Stile der zeitgenössischen Amtsbücher geschrieben, wobei die Schrift allerdings unterschiedliche Grade der Stilisierung bzw. Individualisierung aufweist. Die Schreiberhände wechseln auf

11 Siehe oben Anm. 2. 
den ersten Blättern der Handschrift zunächst sehr häufig. Die Eintragungen ab dem Sommersemester 1402, dem ersten Semester, in dem Supposita in die Matrikel eingeschrieben wurden (fol. $2^{\text {r }}$ ), bis zum Wintersemester 1415/16 (fol. 10 ${ }^{\mathrm{r}}$ ) sind, jeweils semesterweise, von unterschiedlichen Schreibern eingetragen worden. Hierbei existieren jedoch zwei Ausnahmen: Die Eintragungen für die Semester 1405 II (Dekanat des Henricus Berwart, fol. $2^{\mathrm{va}}$ ) und $1406 \mathrm{I}$ (fol. $2^{\mathrm{vb}}$ ) sind von einer einzigen Hand erfolgt. Gleiches gilt auch für die Einträge im Wintersemester 1411/12 (fol. 6r) und für das Wintersemester 1415/16 (fol. 10 ${ }^{r}$, in denen Johannes Sind(e)rami Dekan der Juristenfakultät war. Ob es sich dabei um solche der jeweiligen Dekane oder um die Handschriften von Kanzleinotaren, die von diesen bestellt worden waren, handelt, muss dahingestellt bleiben.

Eine Abkehr von diesem semesterweisen Eintragungsmodus tritt mit dem Dekanat von Caspar Maiselstein im Sommersemester 1416 (fol. 10v) ein. Von diesem Semester an bis zum Dekanat des Johannes Seld im Sommersemester 1425 (fol. $20^{v}$ ) dürfte eine einzige Hand die Einträge durchgehend vorgenommen haben. Legt man diesen Schriftbefund zugrunde, so kann wohl festgestellt werden, dass die von Paul Uiblein bei den Acta Facultatis Artium beobachtete Praxis der von den Dekanen eigenhändig vorgenommenen Eintragungen für die Juristenmatrikel nicht gilt. ${ }^{12}$

Vielmehr dürften die Dekane der Juridischen Fakultät Kanzleischreiber („Notare“) mit der Matrikelführung betraut haben, welche die zunächst auf losen Blättern aufgezeichneten Einträge in vermutlich vorerst ungebundene Pergament-Quinionen geschrieben haben, welche später zu einem Band zusammengebunden wurden. Im Folgenden sei diese Hand, von der die meisten Eintragungen in die Matrikel stammen, kurz beschrieben. Generell weisen die Eintragungen dieser kursiven Haupthand A einen ziemlich hohen Stilisierungsgrad auf. Der Duktus der Schrift ist aufrecht und weist kaum Brechungen auf. Im allgemeinen Schriftbild überwiegen die Schattenstriche, lediglich bei den An- und Abstrichen sowie bei Kürzungen finden sich vereinzelt Haarstriche, wodurch insgesamt der Eindruck einer eher kontrastarmen Schrift vermittelt wird. An Einzelformen stechen besonders die intensiv ausgeprägten Schwellschäfte bei $s$ und $f$ ins Auge, die bisweilen stark in die Unterlänge reichen können. Auffällig ist auch der Buchstabe $h$, der einen in die Unterlänge gehenden, krallenförmigen Abstrich aufweist. Die gleiche Eigenschaft hat auch das Schluss- $n$. Stark in die Unterlänge greift der spitz auslaufende Schaft von $p$, wodurch Überschreibungen mit der nachfolgenden Zeile vorkommen. Bei den Großbuchstaben ist zunächst das alphaförmige $A$ charakteristisch. Typisch für diesen Schreiber ist auch das $S$, das eine schlingenartige Form aufweist und nahezu das Aussehen der Ziffer 8 annimmt.

Mit dem Dekanat des Paulus de Wienna (1426 II, fol. 21 $1^{\mathrm{v}}-22^{\mathrm{r}}$ ) setzt eine neue Hand ein. Die Eintragungen für das Sommersemester 1427 (Dekan Conradus de Bladeck de Wienna, fol. 22r) stammen ebenso wie diejenigen für das Wintersemester 1427/28 (Dekanat von Conradus de Halstat, fol. $22^{v}$ ) von einem neuen Schreiber.

Vom Sommersemester 1428 (fol. 23r) bis zum Sommersemester 1439 (fol. 29r) ist ein semesterweiser Wechsel der Schreiberhände feststellbar.

Ab dem Wintersemester 1439/40 (Dekanat des Petrus Pachmülner, fol. 29v) bis zum Ende des Kodex im Sommersemester 1442 (Dekanat des Conradus de Halstat, fol. 36r) dürfte erneut eine einzelne Person die Einträge in die Matrikel vorgenommen haben. Auch in diesem Fall wird wohl ein Schreiber mit der Matrikelführung betraut worden sein. Die 
Schrift von Haupthand B ist leicht nach rechts geneigt, ihr Stilisierungsgrad ist geringer als jener der Haupthand A. Das Verhältnis von Haar- und Schattenstrichen ist bei dieser Schrift ausgewogener. An Einzelformen fallen vor allem die besonders stark ausgeprägten Schwellschäfte bei $s$ und $f$ auf, deren Schäfte nach unten hin spitz auslaufen. Die Kralle bei $h$ und Schluss- $n$ ist weniger intensiv ausgeprägt als bei der Handschrift von Schreiber A. Bei den Versalien erscheint $A$ in zweifacher Form: Zum einen alphaförmig, zum anderen doppelstöckig ausgestaltet. Besonders deutlich ausgeprägt ist die Keilform bei $R$.

Abschließend sei auf die mehrfach vorkommenden Nachträge hingewiesen, die wohl zumeist bald nach dem Einschreiben der Intitulationen angefügt wurden. Die meisten dieser Hinzufügungen stammen nicht von den Schreibern der semesterweisen Haupteinträge. Ähnlichkeiten dieser Schriften mit anderen Eintragungen konnten nicht festgestellt werden. Die wichtigsten dieser Nachträge finden sich an folgenden Stellen:
fol. $3^{\mathrm{v}}$, (1408 I):
Johannes Wogawer
fol. 12 $2^{\mathrm{r}},(1417 \mathrm{I}) \quad$ (von späterer Hand, wohl aus dem 16. Jahrhundert): sunt comites
fol. $13^{v}$, (1418 II): de Külsheim
fol. 18v, (1423 I): Mag. Cristoferus Guldein $2 \mathrm{gr}$.
fol. 21v , (1426 II): Matheus de Kulled
fol. 27v, (1432 II): p pauper], postea in anno $49 \mathrm{dt} .2 \mathrm{gr}$.
fol. $34^{\mathrm{v}}$, (1439 II): habet terminum unius anni (durchgestrichen), danach: in effectu postea persolvit.

\section{STATISTIK}

Die folgenden Ausführungen sollen keine sozialgeschichtliche Untersuchung der Besucherschaft an der Wiener Rechtsfakultät bieten ${ }^{13}$, sondern lediglich über die Auszählungen einiger Daten berichten, die aus Universitätsmatrikeln üblicherweise erhoben werden können. Die Matrikel der Wiener Rechtsfakultät verzeichnet sowohl die Namen der neu eintretenden Rechtsstudenten als auch die von Absolventen, die zu Bakkalaren, Lizentiaten oder Doktoren graduiert wurden. Daraus folgt, dass ein und dieselbe Person mehrfach eingetragen sein kann. Statistische Aussagen, bei denen die Person fallbildend ist, setzen die Durchführung eines record-linkage voraus. Dies ist nicht erfolgt, so dass bei den folgenden Häufigkeitsauszählungen nicht Personen, sondern Matrikeleinträge gezählt wurden.

\section{Intitulationen und Promotionen}

Die Eintragungen im ersten Band der Wiener Juristenmatrikel reichen vom Sommersemester 1402 bis zum Sommersemester 1442. In diesem Zeitraum wurden von den Dekanen 1.329 Matrikeleinträge vorgenommen. Davon können 322 Einträge Promotionen zugeordnet werden, 1.007 betreffen einfache Immatrikulationen. Dies ergibt eine mittlere Frequenz von 13,6 Immatrikulationen je Semester, bei einem Minimalwert von 1 und einem Maximum von 34.

13 Vgl. dazu Beat Immenhauser, Wiener Juristen. Studien zur Sozialgeschichte der Juristen an der Universität Wien von 1402 bis 1519 (Lizentiatsarbeit in mittelalterlicher Geschichte, Univ. Bern 1996). Eine überarbeitete und gekürzte Fassung ist erschienen in: Mitteilungen der österreichischen Gesellschaft für Wissenschaftsgeschichte 17 (1997), S. 61-102. 
Fig. 1: Immatrikulationen (nach Semestern) 1402-1442

\begin{tabular}{|c|c|c|c|c|}
\hline Summe & Minimum & Maximum & Mittelwert & Standardabweichung \\
\hline 1007 & 1 & 34 & 13,61 & 7,29 \\
\hline
\end{tabular}

Die Verteilung der Immatrikulationen innerhalb des Untersuchungszeitraumes ist aus Fig. 2 ersichtlich (wegen der Übersichtlichkeit nach Jahren zusammengefasst - die Werte für Sommer- und Wintersemester wurden addiert).

Fig. 2: Immatrikulationen (nach Jahren) 1402-1442

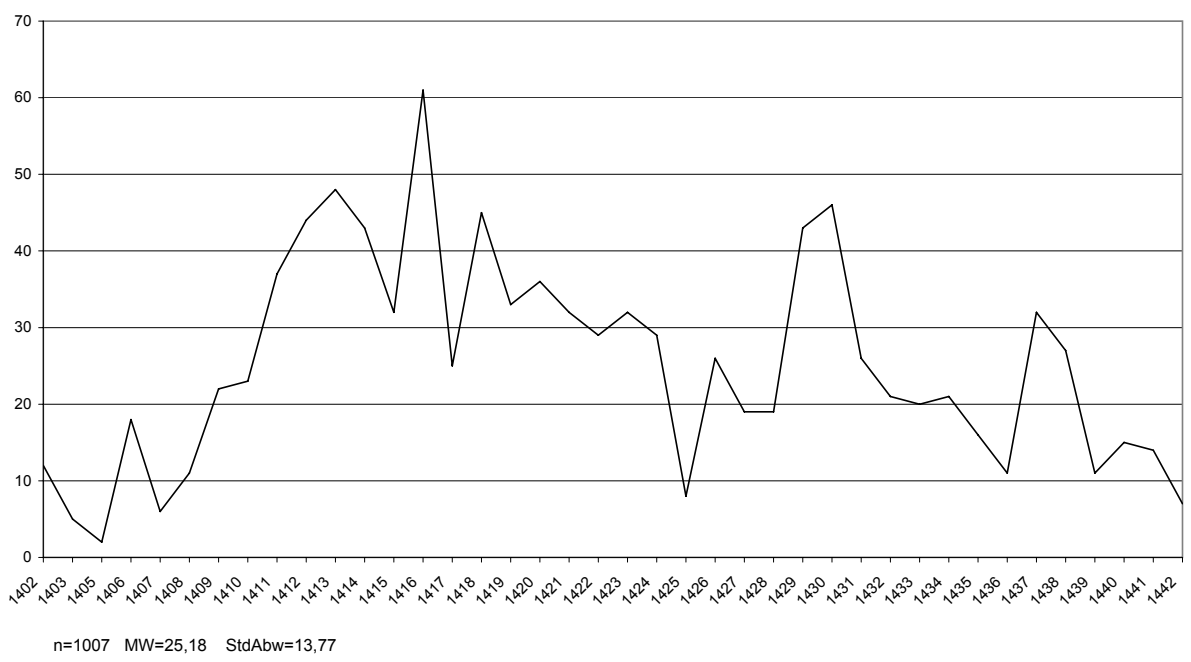

Die Wiener Rechtsfakultät erlebte zu Beginn des 15. Jahrhunderts ein kräftiges Wachstum, was mit dem gesamtuniversitären Besucherstrom in Wien korreliert. ${ }^{14}$ In den Anfangsjahren war die Matrikelführung jedoch nicht immer lückenlos: Von 1403 II bis 1405 I sowie 1406 I und 1407 I fehlen die Eintragungen. Hier handelt es sich nicht um mangelhafte Buchführung, denn auch in der Hauptmatrikel der Universität Wien kann um 1405 ein drastischer Rückgang an Immatrikulationen festgestellt werden. ${ }^{15}$ Die Frequenzeinbrüche um die Jahre 1425 und 1431 sind wohl auf die Wirren der Hussitenkriege zurückzuführen, die in dieser Zeit von Mähren auf österreichische Gebiete nördlich der Donau übergriffen. Auch 1442/43 waren es wahrscheinlich Söldnerscharen aus Mähren und Ungarn, deren Raubzüge die Besucherfrequenz negativ beeinflussten.

Unter den 322 Eintragungen, welche die Promotion zu einem akademischen Grad dokumentieren, entfällt der größte Teil auf die Verleihung des Bakkalaureats: 186 Nennungen (57,8 \% der Promotionen). Es folgen die Erteilung des Lizenziats (82 / 25,5 \%) und des Doktorats (52/16,1\%). Insgesamt beträgt das Verhältnis Immatrikulationen zu

14 Immenhauser, S. 69 ff. Zur Wiener Frequenz vgl. auch Schwinges, S. 61-73.

15 Vgl. dazu Schwinges, S. 67 und 72. Verantwortlich für den Frequenzeinbruch sind wohl Überschwemmungen und Missernten zwischen 1402 und 1406; ebd., S. 72. 
Promotionen ca. 3 zu 1, dies ist eine für das 15. Jahrhundert eher überdurchschnittlich hohe Graduierungsquote. ${ }^{16}$

Bei 323 Eintragungen wurden akademische Grade vermerkt, die bereits an anderen Fakultäten oder Universitäten erworben wurden. Statistisch ins Gewicht fallen dabei nur die Magister der Artistenfakultät: Bei 16,8 \% aller Immatrikulationseintragungen wurde dieser Grad vermerkt. Unter den Promotionsnennungen steigt der Anteil der Artistenmagister auf 30,7\%, bei den Doktoranden allein sogar auf 38,5\%. Die Graduierungsquote der „Magisterstudenten“ liegt demnach deutlich über dem Durchschnitt.

\section{Ständische Gliederung und Taxen}

Einer eingehenden Untersuchung der sozialen Gliederung der Fakultätsbesucher stellen sich mannigfache Hindernisse entgegen: ${ }^{17}$ Standesbezeichnungen in der Matrikel sind häufig unspezifisch, Angaben unvollständig, und die Höhe der entrichteten Matrikeltaxe nicht immer der Standesqualität oder der Taxordnung entsprechend. Wenigstens scheint das Kriterium der Matrikeltaxe als Indikator für sozialen Status noch am ehesten für einen groben Überblick geeignet. Noch vor dem Beginn der eigentlichen Matrikelführung wurde in der vorliegenden Handschrift eine Beschlussfassung zur Taxordnung von 1402 vermerkt. ${ }^{18}$ Demnach hatten die „Normalzahler" bei Beginn des Rechtsstudiums 2 böhmische Groschen zu entrichten, Studenten, die bereits anderswo den Grad eines Juristenbakkalars erworben hatten, 3 Groschen, Adelige oder solche, die dem Adel gleichgehalten werden wollten, einen halben Gulden. Dieses war die Norm; die Praxis zeigt, dass eine Vielzahl von Beträgen vermerkt wurde, die diesem Schema nicht entsprechen. Unterschiedliche Münzsorten und Münzqualität sowie schwankender Geldwert mögen hier unter anderem eine Rolle gespielt haben.

Bei den Immatrikulationseinträgen stellen die „Sollzahler“ mit 2 Groschen die größte Gruppe (563 Fälle, 55,9\%). Unter ihnen bilden die Magister der Artistenfakultät mit 107 Fällen das Schwergewicht. Pauperes waren unter den Juristen eine Seltenheit, nur 33 Immatrikulationen (3,3\%) entfallen auf diese Gruppe. ${ }^{19}$ Es erscheint nicht weiter überraschend, dass die Einträge der Pauperes so gut wie keine weiteren Hinweise auf akademische Grade oder Standesqualität aufweisen. Immerhin 31 \% (313 Fälle) aller Einträge können einer Gruppe zugeordnet werden, die mehr als den Sollbetrag von 2 Groschen, aber weniger als den halben Gulden für die „Adelsbank“ entrichtet hat. Auch hier sind Magister der Artistenfakultät mit 53 Fällen stark vertreten; noch zahlreicher sind jedoch die Angehörigen des Klerus: 163 Eintragungen (52\% der Mehrzahler). Stiftsgeistlichkeit (Kanoniker) und der Pfarrklerus (plebani, presbyteri) sind hier insbesondere vertreten. Unter den 2-Groschen-Zahlern bilden Kleriker eine deutlich kleinere Gruppe (87 Eintragungen, $15,5 \%)$.

Matrikeleintragungen mit einer Taxenzahlung von einem halben Gulden oder mehr sind weniger zahlreich: 81 Fälle, ca. $8 \%$ aller Einträge (ohne Promotionen), weisen einen

16 Zur Graduierungsquote an der Wiener Artistenfakultät vgl. Ingrid Matschinegg und Thomas Maisel, Sozialgeschichtliche Analysen zur Wiener Artistenfakultät im 15. und 16. Jahrhundert. In: Mensch Wissenschaft - Magie. Mitteilungen der österreichischen Gesellschaft für Wissenschaftsgeschichte 20 (2000), S. 135-137.

17 Vgl. dazu Immenhauser, S. 84 ff.

18 MFJ 1401 II (1402 März 08). Vgl. unten S. 3 und Abb. S. XXVII.

19 Unter Pauperes wurden hier jene Einträge subsumiert, bei denen entweder „p. “ vermerkt oder eine Taxe von weniger als 2 Groschen bezahlt wurde. 
gemäß Taxordnung von 1402 dem Adelsstand gebührenden Betrag aus. Inwieweit damit auch eine Aussage über den tatsächlichen Adelsanteil vorliegt, muss an dieser Stelle offen bleiben. ${ }^{20}$ Eine eindeutige Adelstitulatur (comes, nobilis etc.) ist jedenfalls sehr selten. Die am häufigsten in dieser Gruppe vorgefundenen Titulaturen beziehen sich vielmehr auf die Stiftsgeistlichkeit (34 Einträge), bei der adeliger Stand ja oft genug ein Zugangskriterium bildete. Dass Adelstitel bei der universitären „Buchführung“ häufig unterschlagen wurden, kann in spätmittelalterlichen Universitätsquellen (Matrikeln, Acta Facultatis) immer wieder beobachtet werden und stellt daher keine Besonderheit der Juristenmatrikel dar. ${ }^{21}$

\section{Regionale Herkunft der Intitulierten}

Bei 803 der 1.007 Immatrikulationseintragungen findet man Herkunftsangaben, für die eine eindeutige Zuordnung möglich ist. Der überwiegende Teil der Besucherschaft stammte aus Regionen, die der Rheinischen Nation zugerechnet werden können, nämlich aus Süd- und Südwestdeutschland (29,9\%), vor allem aus Orten, die im bayerischfränkischen Raum lokalisiert werden konnten (23,2\%). Das oberdeutsche Einzugsgebiet der Donau war für das Wiener Studium insgesamt ein Kernraum des Universitätsbesuchs, sodass die Juristenscholaren in dieser Hinsicht vom Gesamtbild kaum abweichen. ${ }^{22} \mathrm{Als}$ weiterer Rekrutierungsraum von Bedeutung werden die österreichischen Länder erkennbar, insbesondere das heutige Ober- und Niederösterreich (inklusive Wien 12,4\%) sowie die Steiermark (ohne die heute zu Slowenien gehörenden Gebiete 3,1\%). Dem heutigen Österreich können alles in allem 20,5\% der Einträge zugerechnet werden. Wien selbst ist als Herkunftsort quantitativ ohne Bedeutung, was durchaus dem Befund aus der Hauptmatrikel der Universität entspricht. ${ }^{23}$ Als dritter großer Einzugsraum werden jene Länder erkennbar, welche im 15. Jahrhundert der ungarischen Krone zugerechnet werden können (inklusive heutige Slowakei und Siebenbürgen, 16,4\%). Fast die Hälfte dieser Eintragungen lassen sich Siebenbürgen zuordnen, das als Rekrutierungsraum für Juristen an der Wiener Universität herausragende Bedeutung hatte. Dies wird noch deutlicher, wenn die Graduierungsquoten nach Herkunftsregionen verglichen werden: Bei Siebenbürgern

20 Immenhauser, S. 86, berechnet für den Adel einen deutlich niedrigeren Anteil, weil er als Untergrenze für die Adelstaxe $1 \mathrm{fl}$. einsetzt. Wie dies jedoch mit der Taxordnung von 1402 in Einklang zu bringen ist, bleibt ungeklärt. Auch in einer Taxordnung von 1502 (Statutenbuch der Jur. Fakultät, UAW Cod. J 9, fol. $1^{\text {r) }}$ wird 1 fl. zwar für Herzöge und Grafen vorgeschrieben, von einem „baro“ wurden aber nur 4 sol. verlangt, was wohl in etwa mit einem halben Gulden gleichgesetzt werden kann. Der „simplex nobilis“ musste gar nur 60 d. ( $\approx 2$ sol.) bei der Immatrikulation entrichten. Geht man davon aus, dass hier eine auch schon früher geübte Praxis festgeschrieben wurde, so wird man auch in der Gruppe der Mehrzahler (über 2 gr. und unter einem halben Gulden) eine unbestimmte Zahl von „einfachen“ Adeligen annehmen müssen.

21 Vgl. Christian Lackner, Adel und Studium. Adelige Studenten aus den habsburgischen Ländern an der Universität Wien im 15. Jahrhundert. In: Festschrift Heide Dienst zum 65. Geburtstag, hg. v. Anton Eggendorfer, Christian Lackner und Willibald Rosner (= Forschungen zur Landeskunde von Niederösterreich 30, St. Pölten 2004), S. 71-92, insbes. S. 75. Lackners Untersuchung hat ergeben, dass die Aussage „(...) die österreichischen Eliten mieden das landeseigene Studium ganz offensichtlich“ (IMmenHausER, S. 100) in dieser zugespitzten Form nicht aufrecht zu erhalten ist.

22 Vgl. dazu Christan Hebeisen u. Thomas Schmid, De Zusato, Coloniensis diocesis. Über Herkunftsräume armer Universitätsbesucher im Alten Reich (1375 bis 1550). In: Jahrbuch für Universitätsgeschichte 6 (2003), S. 36.

23 Albert Müller, Universitätsbesuch und städtische Herkunft. In: Aspekte der Bildungs- und Universitätsgeschichte, 16.-19. Jahrhundert, hg. v. Kurt MüHLberger und Thomas MAisel (= Schriftenreihe des Universitätsarchivs, Universität Wien, Bd.7, Wien 1993), S. 345. 
verhält sich die Zahl der Graduierungen zu den Eintragungen ca. 2 zu 3, aber auch bei Promotionseinträgen mit dem „restlichen“ Ungarn (inkl. Slowakei) als Herkunftsangabe liegt dieser Wert über dem Durchschnitt (vgl. Kap. 3.1). Gegenteilig verhält sich dieser Trend bei Eintragungen zum süd- und südwestdeutschen Raum: Hier beträgt das Verhältnis Promotionen zu Immatrikulationen lediglich $1 \mathrm{zu} 4$.

Fig. 3: Herkunft bei Immatrikulationen

Fig. 2: Immatrikulationen (nach Jahren) 1402-1442

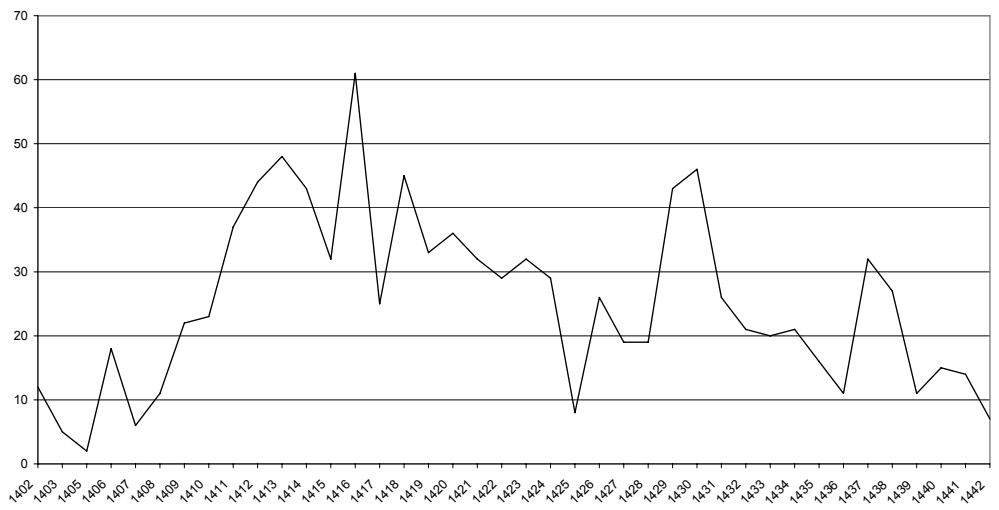

$\mathrm{n}=1007 \quad \mathrm{MW}=25,18 \quad \mathrm{StdAbW}=13,77$

6,5\% aller Immatrikulationen waren Ortschaften in Böhmen und Mähren zuordenbar. Besonderes Gewicht besaß jedoch Schlesien, das mit dem Bistumssitz Breslau die häufigsten Nennungen eines Ortsnamens unter allen Matrikeleinträgen aufweist $(n=16)$. $11,2 \%$ aller Einträge können im heutigen Polen lokalisiert werden, wobei die Schlesier das Hauptkontingent stellen. ${ }^{24}$ Ihre Graduierungsquote reicht jedoch an die der Siebenbürger nicht heran (ca. $1 \mathrm{zu}$ 4).

Eine mögliche Erklärung für die niedrige Graduierungsquote von Studenten aus oberdeutschen Territorien mag sein, dass unter den Zahlern der Adelstaxe diese Herkunftsregionen überproportional hoch vertreten sind (fast $30 \%$ aus Bayern). Studenten, die bereits über Status verfügten, waren nicht unbedingt auf einen akademischen Grad angewiesen. Eine Zusammenschau der Faktoren Standesqualität (anhand der Taxenhöhe), Graduierung und Herkunftsregion ergibt, knapp gefasst, den Befund, dass aus deutschen Territorien mehr Studenten von Stand die Wiener Rechtsfakultät frequentierten, ohne

24 Immenhauser, S. 82 erkennt wohl zu Recht, dass hier die Stiftung der sogenannten Schlesier- oder Silesenburse durch den Breslauer Domherrn Nikolaus von Gleiwitz eine zentrale Rolle spielt. Zur Silesenburse siehe Kurt MüHlberger, Wiener Studentenbursen und Kodreien im Wandel vom 15. zum 16. Jahrhundert. In: Aspekte der Bildungs- und Universitätsgeschichte, (wie Anm. 23), S. 176 f. 


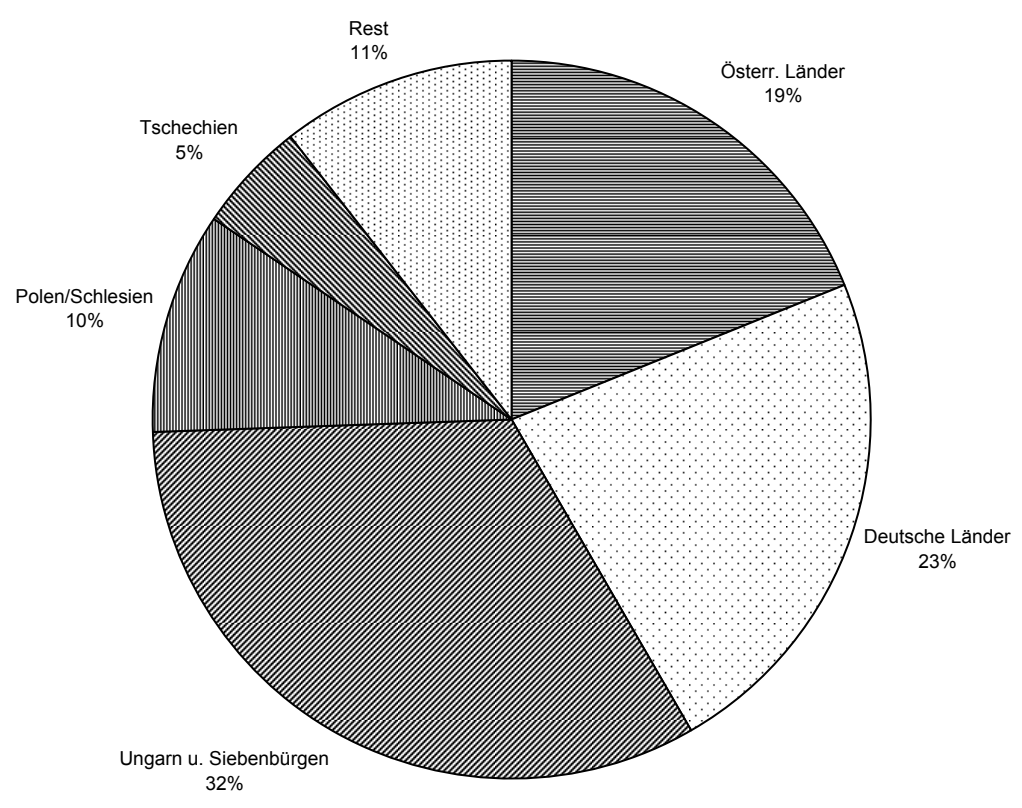

jedoch einen akademischen Grad zu erwerben (was sie, wenn überhaupt, vielleicht an den renommierten italienischen Universitäten taten), während aus dem östlichen Rekrutierungsraum, insbesondere Siebenbürgen, Studenten angezogen wurden, für die ein in Wien erworbener juristischer Grad attraktiv war.

Andere als die bisher erwähnten europäischen Länder haben als Herkunftsgebiete für Juristenscholaren zwischen 1402 und 1442 quantitativ kaum Bedeutung. Einzelne Studenten kamen jedoch auch aus verhältnismäßig weit entfernten Gebieten, wie etwa Spanien, den Niederlanden oder Ostpreußen. Frankreich und Italien spielten als Herkunftsräume für Studenten in Wien ebenfalls kaum eine Rolle, was aufgrund der dort vorhandenen „gewachsenen“, altehrwürdigen Rechtsfakultäten nicht verwundern kann.

\section{GRUndsäTZE DER Edition}

Für die Textgestaltung wurden im Wesentlichen die von Walter Heinemeyer herausgegebenen Richtlinien für die Edition mittelalterlicher Amtsbücher herangezogen. ${ }^{25}$ Die Wiedergabe der Eigennamen erfolgt buchstabengetreu, aber mit großem Anfangsbuchstaben. Weiters wurde die durchgehende Normalisierung von „“" und „j" bzw. „u“ und „ „ae“, etc.).

25 Richtlinien für die Edition mittelalterlicher Amtsbücher. In: Richtlinien für die Edition landesgeschichtlicher Quellen, hrsg. von Walter Heinemeyer (Marburg - Köln 1978), S. 17-23 
Standes- und Berufsbezeichnungen sowie akademische Grade werden klein geschrieben, außer sie sind dem Eigennamen vorangestellt. Zusätze und Ergänzungen der Editoren werden durch eckige Klammern [] oder Kursivdruck gekennzeichnet.

Unsichere Lesarten, Emendationen oder Nachträge werden in Fußnoten angegeben, während spitze Klammern $<>$ auf Rasuren verweisen.

Die Foliierung wird im Text am linken Rand in Kursivdruck angegeben. Ebenso erscheint die für jedes Studienjahr durchlaufende Namenszählung (von 5 zu 5 Namen) am linken Rand. Die in der Edition aufscheinende Zählung ist kein Bestandteil des Quellentextes $^{26}$. Der Originaltext ist nur auf den Blättern $2^{r}-5^{r}$ in Spalten geschrieben, was in der Edition nicht übernommen wurde.

Zahlenangaben in römischen Zahlzeichen bzw. arabischen Ziffern erfolgen vorlagengetreu. Abkürzungen wurden stillschweigend aufgelöst, wenn dies einwandfrei möglich war, ansonsten wurden sie durch eckige Klammern [ ] oder Fußnoten als unsichere Auflösung bzw. als nicht aufgelöst gekennzeichnet. Einige immer wiederkehrende Kürzungen wurden beibehalten. ${ }^{27}$

\section{Die Dekane ${ }^{28}$}

\begin{tabular}{|c|c|c|}
\hline 1401 & II & N. N. \\
\hline 1402 & I & Johannes (de Garsonibus) de Venetiis, decr. dr. ${ }^{29}$ \\
\hline 1402 & II & N. N. \\
\hline \multirow[t]{2}{*}{1403} & April 14 & Gislerus Dobberkow, decr. dr. \\
\hline & II & N. N. \\
\hline \multirow[t]{2}{*}{1404} & I & N. N. \\
\hline & II & N. N. \\
\hline \multirow[t]{2}{*}{1405} & I & N. N. \\
\hline & Oktober 13 & Henricus Bernstein, mag. \\
\hline \multirow[t]{2}{*}{1406} & I & N. N. \\
\hline & Oktober c. 13 & Johannes Sindrami, decr. dr. \\
\hline \multirow[t]{2}{*}{1407} & I & Lienhardus Schawr (?) \\
\hline & Oktober c. 13 & Henricus Kitzbühel, decr. dr. \\
\hline \multirow[t]{2}{*}{1408} & April c. 23 & Casparus Maiselstein, decr. dr. \\
\hline & Oktober c. 13 & Michael Azmannsbach, lic. in decretis \\
\hline \multirow[t]{2}{*}{1409} & April c. 23 & Johannes Sindrami, decr. dr. \\
\hline & Oktober c. 13 & Casparus Maiselstein, decr. dr. \\
\hline \multirow[t]{2}{*}{1410} & April c. 23 & Henricus Kitzbühel, decr. dr. \\
\hline & Oktober c. 13 & Michael Azmannsbach, lic. in decretis \\
\hline 1411 & $\begin{array}{l}\text { April } 14 \\
\text { Oktober n. } 14\end{array}$ & $\begin{array}{l}\text { Gerardus Vischbeck, mag., decr. dr., canonicus Vienn. } \\
\text { Johannes Sindrami, mag., decr. dr. }\end{array}$ \\
\hline
\end{tabular}

26 S. oben Kapitel II., 3. Foliierung.

27 S. Abkürzungsverzeichnis, S. XXV.

28 Zur Frage der Amtsübernahme sie oben Anm. 7 und 8.

29 Der Beginn des Dekanats des Johannes de Venetiis ist quellenmäßig nicht belegt. Die fragliche Zuordnung zum Sommersemester 1402 findet sich bei Asснвасн I, 304, 586, jedoch ohne Quellenbeleg. Die betreffenden Stellen vgl. bei Uiblein, AFA I, S. 529; Goldmann, S. 118 m. Anm. 5. Vgl. auch Kink 1, 171. 
1412 April 14

Oktober 13

1413 April 14

Oktober 13

1414 April 14

Oktober 13

1415 April 14

Oktober 13

1416 April 14

Oktober 13

1417 April 14

Oktober 13

1418 April 14

Oktober 13

1419 April 14

Oktober 13

1420 April 14

Oktober 13

$1421 \quad$ April 14

Oktober 13

1422 April 14

Oktober 13

1423 April 14

Oktober 13

1424 April 14

Oktober 13

1425 April n. 14

Oktober n. 13

1426 April n. 14

Oktober 13

1427 April n. 14

Oktober n. 13

1428 April n. 14

Oktober n. 15

1429 April c. 14

Oktober n. 13

$1430 \quad$ April n. 14

Oktober n. 13

$1431 \quad$ April n. 14

Oktober n. 13

1432 April 28

Oktober n. 13

1433 April n. 14

Oktober n. 13

1434 April 25
Johannes Schallermann de Westphalia, mag., decr. dr.

Petrus Deckinger de Vienna, mag., lic. in decr.

Wilhelmus Kirchner de Constancia, mag., decr. dr.

Conradus Seglauer

Casparus Maiselstein, decr. dr.

Michael Azmannsbach, lic. in decr.

Gerardus Vischbeck, mag., decr. dr.

Johannes Sindrami, mag., decr. dr.

Casparus Maiselstein, mag., decr. dr.

Johannes Schallermann de Westphalia, mag., decr. dr.

Conradus Rawching, lic. in decr.

Gerardus Vischbeck, mag., decr. dr.

Fridericus Krafft, decr. dr.

Petrus Deckinger de Vienna, mag., lic. in decr.

Mathias Regelshouer de Herrieden, lic. in decr.

Casparus Maiselstein, mag., decr. dr.

Johannes Sindrami, decr. dr.

Paulus Paewrl de Vienna, mag., decr. dr.

Johannes Gwerleich, decr. dr.

Johannes Schallermann de Westphalia, decr. dr.

Johannes de Perchtoldsdorf, decr. dr., can. Neuburgensis

Nikolaus Stock de Maiori Glogouia, mag., decr. dr.,

Conradus de Hallstatt, mag., decr. dr.

Johannes Hess de Ysenach, lic. in decr.

Casparus Maiselstein, mag., decr. dr.

Petrus Pachmulner de Eberspewnt, mag., lic. in decr.

Johannes Seld(e), decr. dr.

Paulus de Vienna, mag., decr. dr.

Johannes Gwerleich, decr. dr.

Thomas Oeder de Aspach, mag., lic. in decr.

Conradus Bladek de Vienna, mag., lic. in decr.

Conradus de Hallstatt, mag., decr. dr.

Ulricus de Weyssenburga, mag. lic. in decr.

Paulus de Vienna, mag., decr. dr.

Johannes Seld(e), decr. dr.

Conradus de Hallstatt, mag., decr. dr.

Johannes Gwerleich, decr. dr.

(G)erhardus Herrant, mag., lic. in decr.

Petrus Pachmulner de Eberspewnt, mag., decr. dr.

Paulus de Vienna, mag., decr. dr.

Johannes Gwerleich, mag., decr. dr.

Petrus Nowag,. decr. dr., can. eccl. Transyluane

Hartungus des Cappel, utriusque iuris dr., can. eccl. Patav.

(G)erhardus Herrant, mag., decr. dr., officialis curie Patav.

Conradus de Hallstatt, mag., decr. dr.

Oktober n. 13 Petrus Pachmulner de Eberspewnt, mag., decr. dr. 
Johannes de Eych, utriusque iuris dr.

Oktober c. 13 Johannes Gwerleich, mag., decr. dr.

1436 April c. 14 Johannes Polczmacher, decr. dr.

Nov. c.- 25 Conradus de Hallstatt, mag., decr. dr.

1437 April n. $14 \quad$ Johannes Seld(e), decr. dr.

Oktober n. 13 Johannes de Eych, utriusque iuris dr.

1438 Mai $10 \quad$ Petrus Pachmulner de Eberspewnt, mag., decr. dr. Oktober 23 Martinus de Walthausen, ven. et egr. vir

1439 April $14 \quad$ Johannes Polczmacher, decr. dr. Oktober n. 13 Johannes Seld(e), decr. dr.

1440 April c. 14 Martinus de Walthausen, decr. dr., ven. et egr. vir Oktober c. 13 Conradus de Hallstatt, mag., decr. dr.

1441 April c. $14 \quad$ Johannes Polczmacher, decr. dr. Oktober c. 13 Petrus Pachmulner de Eberspewnt, mag., decr. dr.

1442 April c. 14 Martinus de Walthausen, decr. dr., ven. et egr. vir

\section{AbKürzungen und Hilfsmittel}

\section{Kurzzitate (Quellen und Literatur)}

Aschbach Josef Aschbach, Geschichte der Wiener Universität im ersten Jahrhunderte ihres Bestehens. Festschrift ihrer fünfhundertjährigen Gründungsfeier (Wien 1865).

$\mathrm{AU}$

CoIng

ERDő

GaLl, MUW I Die Matrikel der Universität Wien, 1. Band: 1377-1450, bearb. v. Franz GaLL, etc. (Graz/Köln 1956).

Gaudemet Jean Gaudemet, La formation du droit canonique médiéval (= Collected studies series 111, London 1980).

Goldmann Artur Goldmann, Die Universität 1529-1740. In: Geschichte der Stadt Wien, hrsg. vom Alterthumsverein zu Wien, 6. Band, red. Anton Mayer (Wien 1918) 1-205, 6 Tafeln, 18 Fig.; (auch als Voraus-Separatabdruck 1917 erschienen). 
Immenhauser Beat Immenhauser, Wiener Juristen. Studien zur Sozialgeschichte der Juristen an der Universität Wien von 1402 bis 1519. In: Mitteilungen der österreichischen Gesellschaft für Wissenschaftsgeschichte 17 (1997) S. $61-102$

KINK Rudolf KInK, Geschichte der kaiserlichen Universität zu Wien. Im Auftrage des k.k. Ministers für Cultus und Unterricht, Leo Grafen von Thun. 1. Band, 1. Teil: Geschichtliche Darstellung der Entstehung und Entwicklung der Universität bis zur Neuzeit. Sammt urkundlichen Beilagen. (Wien 1854); - 1. Band, 2. Teil: Urkundliche Beilagen (Wien 1854) 327 S.; - 2. Band: Statutenbuch der Universität (Wien 1854).

MFJ Matricula Facultatis Juristarum

NH Natio Hungarica

NR Natio Rhenana (Rhenensium)

Plöchl Willibald M. Plöchl, Das Kirchenrecht in der ältesten Studien- und Prüfungsordnung der Wiener Rechtsfakultät. Ein Beitrag zur Geschichte der Kirchenrechtswissenschaft. In: Studia Gratiana 2 (1954) 567-581.

Santifaller Leo Santifaller, Das Brixner Domkapitel in seiner persönlichen Zusammensetzung im Mittelalter (= Schlern Schriften 7, Innsbruck 1924)

Schrauf, AFM Acta facultatis medicae universitatis Vindobonensis. Auf Veranlassung des Medicinischen Doctorenkollegiums aus der Originalhandschrift herausgegeben Band 1-2, hrsg. von Karl Schrauf (Wien 1894-1899)

Schrauf, NH Die Matrikel der Ungarischen Nation an der Wiener Universität 14531630, ed. Karl Schrauf (Wien 1902).

Schwinges Rainer Ch. Schwinges, Deutsche Universitätsbesucher im 14. und 15. Jahrhundert. Studien zur Sozialgeschichte des alten Reiches (= Veröffentlichungen des Instituts für europäische Geschichte Mainz 123, Stuttgart 1986).

Uiblein, AFA I Acta Facultatis Artium Universitatis Vindobonensis 1385-1416, hg. von Paul Uiblein (= Publikationen des IfÖG VI. Reihe: Quellen zur Geschichte der Universität Wien, 2. Abteilung, Graz/Wien/Köln 1968).

Uiblein, AFTh Die Akten der Theologischen Fakultät der Universität Wien, 13961508, 2 Bände, hg. von Paul Uiblein (Wien 1978).

Uiblein, Mittelalterliches Studium

Paul Uiblein, Mittelalterliches Studium an der Wiener Artistenfakultät. 
Kommentar zu den Acta Facultatis Artium Universitatis Vindobonensis 1385-1416 (= Schriftenreihe des Universitätsarchivs, Universität Wien 4, Wien $\left.{ }^{2} 1995\right)$.

\section{Verzeichnis der verwendeten Ortsnamenbücher und Karten}

Milan Orožen Adamič, Drago Perko, Drago Kladnik, Priročni krajevni leksikon Slovenije (Ljubljana 1996).

Allgemeines Postlexikon der im Reichsrate vertretenen Königreiche und Länder und des Fürstentums Liechtenstein (Wien 1906).

Karl BosL (Hrsg.), Handbuch der historischen Stätten Deutschlands 7, Bayern. Stuttgart, 2. Aufl. 1965).

Duden - Wörterbuch geographischer Namen. Europa ohne Sowjetunion (Mannheim 1966).

Johann Georg Theodor Graesse, Friedrich Benedict, Helmut Plechl, Orbis Latinus. Lexikon lateinischer geographischer Namen des Mittelalters und der Neuzeit, 3 Bde. (Braunschweig 1972).

Handbuch der historischen Stätten Österreich 1: Donauländer und Burgenland, hrsg. v. Karl Lechner (Stuttgart 1970); 2: Alpenländer mit Südtirol, hrsg. v. Franz Huter, 2. Aufl. (Stuttgart 1978).

Josef von Kendler und Karl von Kendler, Orts- und Verkehrs-Lexikon von ÖsterreichUngarn (Wien ${ }^{3} 1905$ ).

Egon Kühebacher, Die Ortsnamen Südtirols und ihre Geschichte. 3 Bde. (Bozen $\left.{ }^{2} 1995-2000\right)$.

György LeLKes (Hrsg.), Magyar helységnév-azonosító szótár (Baja 21998).

Helmut Opıtz (Red.), Müllers großes deutsches Ortsbuch. Bundesrepublik Deutschland. Vollständiges Gemeindelexikon (München ${ }^{28} 2003$ ).

PolEn. Poland, Pologne ; Umgebungskarte, Stadtpläne Warszawa, Gdansk, Szczecin, Verzeichnis der Orte und Sehenswürdigkeiten, Entfernungstabelle, Reiseinformationen ; Ortsnamen polnisch - deutsch. 1:600 000 (= Hildebrand's Urlaubskarte, Frankfurt/ Main 1999).

Claudia Schnieper, Robert Schnieper, Neues schweizerisches Ortslexikon. Nouveau dictionnaire géographique de la Suisse (München - Luzern ${ }^{23} 1983$ ).

Slowenien, Kroatien, Bosnien-Herzegowina, Serbien und Montenegro, Makedonien, Albanien. Slovenija, Hrvatska, Bosna i Hercegovina, Srbija i Crna Gora, Makedonija, Shqipëria mit Ortsverzeichnis und Cityplänen Bled, Belgrad, Dubrovnik, Pula, Rijeka, Split, Trogir, Zadar mit Hervorhebung der Sehenswürdigkeiten. 1:750.000 (Ostfildern 2001) [Landkarte].

Heribert STURm (Hrsg.), Ortslexikon der böhmischen Länder 1910-1965 (München Wien 1983)

Hugo Weczerka (Hrsg.), Schlesien. (= Handbuch der historischen Stätten Deutschlands 15, Stuttgart 1977).

Hans Zikmund (Bearb.), Duden - Wörterbuch geographischer Namen des Baltikums und der Gemeinschaft Unabhängiger Staaten (GUS) (Mannheim - Leipzig - Wien Zürich 2000). 


\section{Abkürzungen im Text und in den Registern}

A

Natio Austriaca, Österreichische Nation

a. H. andere(r) Hand

archidiac.

archidiaconus

art.

artes (in Verbindungen)

b. a.

baccalarius artium

bacc. in decr.

baccalarius in decretis

Bez.

Bezirk

$c(a)$.

circa

can.

canonicus

can. reg.

canonicus regularis

cap.

capitulum

card.

cardinalis

d.

denarius, Pfennig

decan.

decanus

decr. dr.

decretorum doctor

Dep.

Departement

dom.

dominus

dr. utr. iur.

doctor utriusque iuris

dt.

dedit

Dtld.

Deutschland

eccl.

ecclesia

ehem.

ehemalig(-er, -e, -es)

episc.

episcopus

fac.

facultas

fac. iur. can(on). facultas iuris canonici

fl.

florenus, Gulden

franz.

französisch

GB

Gerichtsbezirk

Gem.

Gemeinde

gr.

grossus, Groschen

$\mathrm{H}$

Natio Hungarica, Ungarische Nation

imm.

immatrikuliert

Kant.

Kanton

KG

Katastralgemeinde

Kom.

Komitat

Kr.

Kreis

kreisfr. Stadt

kreisfreie Stadt

kroat.

kroatisch

Ktn.

Kärnten

lb.

librum, Pfund

lic. a.

licentiatus artium

lic. in a.

licentiatus in artibus

lic. in decr.

licentiatus in decretis

m. a.

magister artium

mag.

magister 
med.

n.

NÖ

Ö

OÖ

OPraem

OSB

p.

pleb.

poln.

prep.

proc.

prof.

Prov.

$\mathrm{R}$

RB (Rgb.)

Rh.

rumän.

S

s. H.

s.

Sbg.

serb.

slowak.

slowen.

sol.

Stmk.

substit.

$\mathrm{T}$

theol.

tschech.

ung.

ungar.

Univ.

Verw.

viell. medicina

nach

Niederösterreich

Österreich

Oberösterreich

ordinis Praemonstratensis

ordinis sancti Benedicti

pauper

plebanus

polnisch

prepositus

procurator

professus

Provinz

Natio Rhenensium, Rheinische Nation

Regierungsbezirk

Rhenensium, Rhenensis

rumänisch

Natio Saxonica, Sächsische Nation

selbe(r) Hand

siehe; sanctus

Salzburg

serbisch

slowakisch

slowenisch

solidus, Schilling

Steiermark

substitutus

Tirol

theologia

tschechisch

ungaricus, magyarisch

ungarisch

universitas, Universität

Verwaltung

vielleicht 


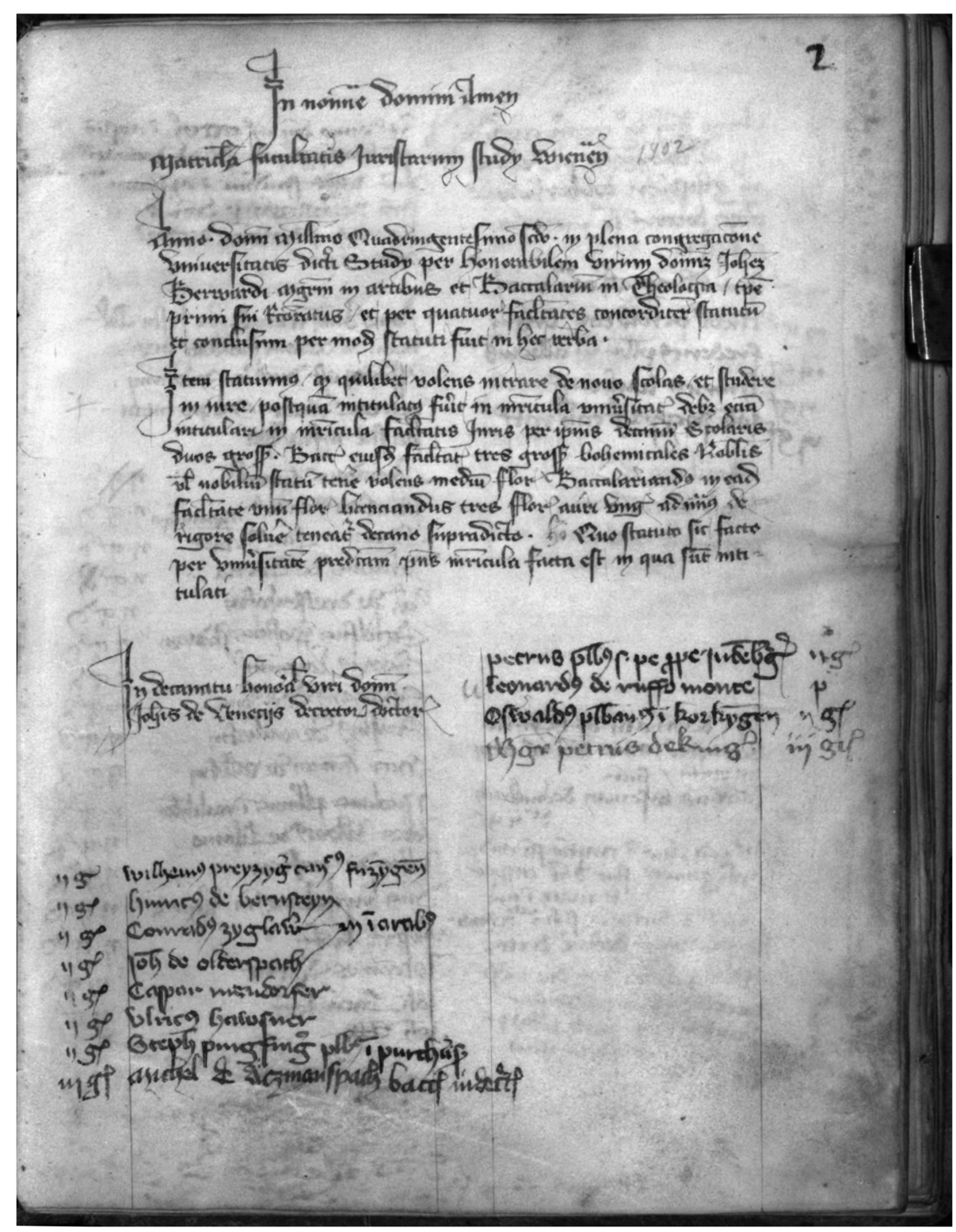

Incipit der Juristenmatrikel, 1402. In nomine Domini Amen.

Matricula facultatis iuristarum studii Wiennensis: Beschluss der Universitätsversammlung betreffend Immatrikulationspflicht und Matrikeltaxen. (UAW, Cod. J 01, fol. 2r) 


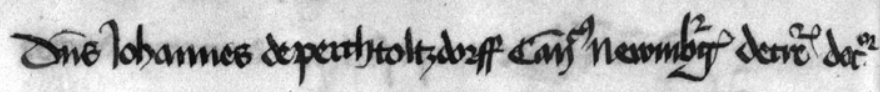

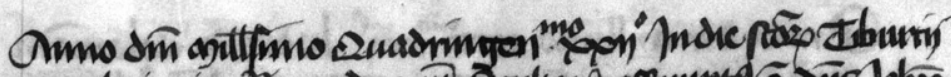

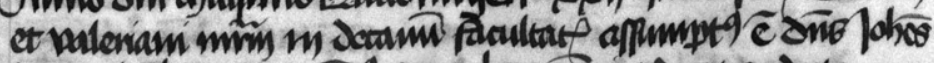

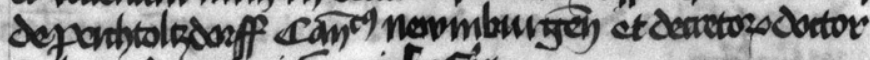

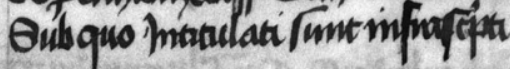

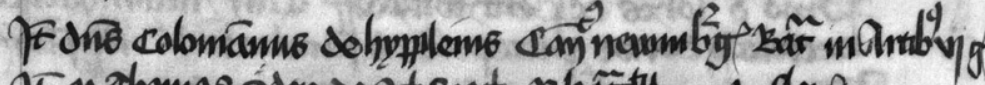

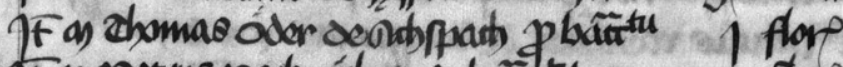

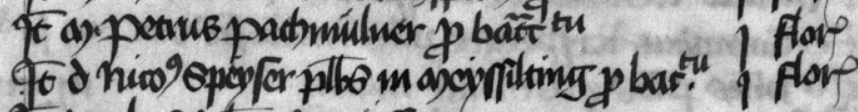
c) Jarobus johre de anifión

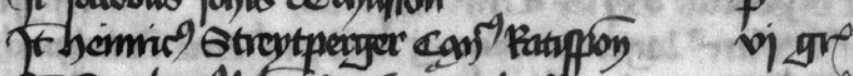

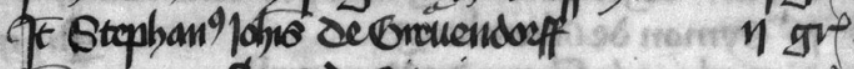
IE a) mensqtans de situmia

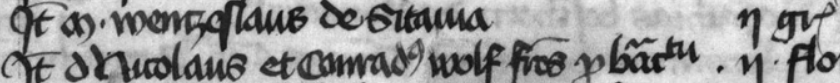

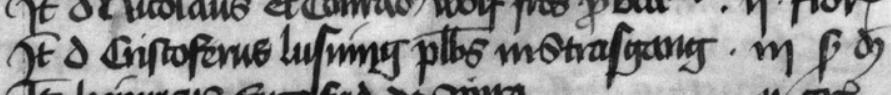
Thémmans Gujelind oespina

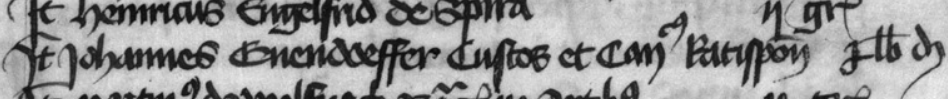

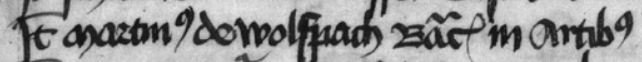

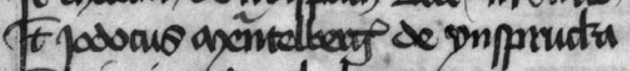
(t) héminar de obobuigr to polos seknaming t 0 .

Ed ar anom dexypary

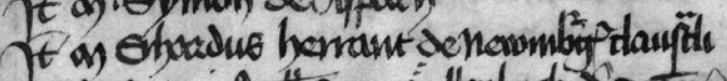

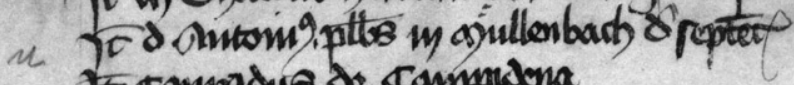
y gor प 910 ing $\eta \pi r^{2}$ fflove $1.5 v^{\circ}$

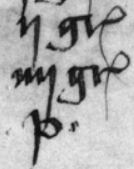

Matrikelführung unter dem Dekan Johannes de Perchtoldsdorf anno 1422 (UAW, Cod. J 01, fol. 17v) 
Text der Matrikel 1402-1442 

fol. $2^{r}$

[1401 II]

In nomine Domini Amen.

Matricula facultatis iuristarum studii Wiennensis

(1402 März 08) ${ }^{1} \mathrm{Anno}^{2}$ Domini millesimo quadringentesimo secundo in plena congregacione universitatis dicti studii per honorabilem virum dominum Johannem Berwardi magistrum in artibus et baccalarium in theologia tempore primi sui rectoratus et per quatuor facultates concorditer statutum et conclusum per modum statuti fuit in hec verba:

Item statuimus, quod quilibet volens intrare de novo scolas et studere in iure, postquam intitulatus fuerit in matricula universitatis, debet eciam intitulari in matricula facultatis iuris per ipsius decanum: Scolaris duos grossos, baccalarius eiusdem facultatis tres grossos Bohemicales, nobilis vel nobilium statum tenere volens medium florenum, baccalariandus in eadem facultate unum florenum, licenciandus tres florenos auri Ungaricales ad minus de rigore solvere teneatur decano supradicto. ${ }^{3}$ Quo $^{4}$ statuto sic facto per universitatem predictam presens matricula facta est, in qua sunt intitulati:

fol. $2^{\text {ra }}$

$[1402 \mathrm{I}]$

\section{In decanatu honorabilis viri domini Johannis de Veneciis ${ }^{5}$ decretorum doctoris:}

Wilhelmus Preyzyger canonicus Frizyngensis 2 gr. $^{6}$

$\left.\begin{array}{l}\text { Hinricus de Bernsteyn } \\ \text { Conradus Zyglawer } \\ \text { Johannes de Olderspach }\end{array}\right\}$ mag. in artibus $\left\{\begin{array}{l}2 \text { gr. } \\ 2 \text { gr. } \\ 2 \text { gr. }\end{array}\right.$

5 Caspar Mendorfer 2 gr.

Vlricus Hawsner 2 gr.

Stephanus Purgfinger plebanus in Purchusen 2 gr.

Michel de Åczmanspach baccalarius in decretis 3 gr. $^{7}$

fol. $2^{\text {rb }}$

Petrus plebanus Sancti Petri prope Judenburgam 2 gr.

1 Vgl. zur Datierung anhand der im Folgenden zitierten Universitätsversammlung Uiblein, AFA I, 206 f., und AU I/2, UAW, Cod. R 1.2, fol. $20^{\text {rv }}$.

2 Von „In nomine“ bis „Anno“ mit roter Tinte geschrieben.

3 Von „Item statuimus ...“ an ist diese Passage inhaltlich übereinstimmend mit Acta univ. AU II, UAW Cod. R 1.2, fol. 20v , und Statutenbuch der Jur. Fakultät, UAW Cod. J 9, fol. 17v․ Vgl. auch KInk 2, S. 233 f., Nr. 17.

4 Davor getilgt „Ho“.

5 Zur Datierung des Dekanats von Johannes de Veneciis vgl. Asснвасн, S. 304 f., 568.

6 Auf fol. $2^{\text {ra }}$ und fol. $2^{\text {va }}$ wurden die Taxen am linken Rand der Spalten eingetragen.

7 Gesamter Eintrag von a. H. 
10 Leonardus de Ruffo Monte p.

Oswaldus plebanus in Korkyngen 2 gr.

Mag. Petrus Dekinger 3 gr. $^{8}$

fol. $2^{v a}$

[1403 I]

(1403 April 14 - Oktober 13) Anno Domini Mo CCCCIII' a [festo] Tyburcii usque ad [festum] Colomanni in decanatu Ghiseleri Dobberkow decretorum doctoris sunt intitulati subscripti:

Hinricus Kranpergher canonicus Ratisponensis 3 gr.

Nicolaus Perold canonicus Brixinensis 3 gr.

Fredericus plebanus in Albeins 3 gr.

Hinricus Wichtorff 2 gr.

5 Johannes Streyperger canonicus Ratisponensis 6 gr.

[1405 II]

Item in decanatu magistri Heynrici Bernsteyn anno Domini $\mathrm{M}^{\circ} \mathrm{CCCCV}^{\circ}$ intytulatus fuit:

Jacobus Hofeman de Onalbach dt. 2 gr.

(1405 Dezember 01) Item eodem anno in crastino sancti Andree Apostoli promotus fuit dominus Caspar licenciatus in iure canonico in doctorem sub domino Leonardo Schawer decretorum doctore.

(1405 Dezember 17) Item eodem anno XVII die mensis Decembris incorporatus fuit facultati mag. Gerhardus etc. ut supra in primo folio huius libri. ${ }^{9}$

fol. $1^{v}$

In decanatu magistri Heynrici Pernstaýn.

(1405 Dezember 17) Anno Domini millesimo quadringentesimo quinto XVII ${ }^{10}$ die mensis Decembris hora vespertina facta plena congregacione facultatis in stuba communi scole iuristarum unanimiter nulli contradicente ex evidenti necessitate scilicet carencia doctorum regencium dispensatum fuit cum magistro Gerhardo Vischpekh ${ }^{11}$ canonico Sancti Stefani ${ }^{12}$, decretorum doctore, qui pro tunc regenciam assumere intendebat, super incorporacione dicti magistri Gerhardi facultati supradicte sub tali forma, quod non obstante quodam statuto in rubrica De noviciis - quo cavetur, quod doctor aliene universitatis de novo superveniens ${ }^{13}$

8 Gesamter Eintrag von a. H. Der Rest der Spalte wurde frei gelassen.

9 Der nachfolgende Einschub befindet sich in der Vorlage auf fol. $1^{\mathrm{v}}$. Er bezieht sich auf die hier vermerkte Inkorporation des Mag. Gerhardus Vischpekh.

10 Von anderer Hand über teilweise radiertem „X et VII ${ }^{a}$ “ eingetragen.

11 Familienname mittels Verweiszeichen am linken Seitenrand von a. H. nachgetragen.

12 „canonico Sancti Stefani“ von a. H. auf freigelassenem Spatium nachgetragen.

13 Siehe KINK 2, 131 
antequam incorporetur, faciet unum actum repetetorium ipse - pro unito et incorporato dicte facultati haberetur ac si repeticionem dictam complevisset.

(1405 Dezember 19) Item anno nativitatis eiusdem loco ubi supra die XIX mensis Decembris facta plena congregacione facultatis ex unanimi consensu omnium ibi presencium dispensatum fuit cum predicto magistro Gerhardo super regenciam videlicet, quod non ${ }^{14} \mathrm{ob}$ [stante] quodam statuto situato sub rubrica De doctoribus ${ }^{15}$, quod incipit: Item quod quilibet doctor legere vel regere volens etc. - quo cavetur quemlibet regentem semel in anno teneri repetere et semel disputare seu loco disputacionis secundario repetere - ut ipse nichilominus, licet nullum predictorum isto anno faceret, censeri deberet regens in facultate iuris propter lectionem ordinariam, quam pro tunc fecit. Conclusum ergo fuit unanimiter dictum magistrum Gerhardum incorporatum ${ }^{16}$ esse facultati actu regentem et pro tunc legentem.

fol. $2^{v b}$

(1406 Februar 04) Item anno Domini $\mathrm{M}^{\circ} \mathrm{CCCCVI}^{\circ}$ in crastino sancti Blasii promoti sunt:

5 ad doctoratum $\} \begin{aligned} & \text { dom. Johannes Syndrami et } \\ & \text { mag. Heinricus Pernstayn }\end{aligned}$

per dominum dictum Leonardum Schawer decretorum doctorem.

[1406 II]

(Ca. 1406 Oktober 13) Anno Domini MCCCCVIo circa festum Colmanni in decanum facultatis iuris assumptus est dominus Johannes Syndrami decretorum doctor, sub quo intitulati sunt infrascripti:

Conradus Trawner 2 gr. dt.

$\left.\begin{array}{l}\text { Johannes canonicus regularis } \\ \text { Georius canonicus regularis }\end{array}\right\}$ Neunburgensis $\left\{\begin{array}{l}2 \text { gr. } \mathrm{dt} . \\ 2 \text { gr. } \mathrm{dt} .\end{array}\right.$

Achacius de Tyrna 2 gr. dt.

5 Johannes de Dyessenhofen 2 gr. dt.

Eglolfus prepositus Ardacensis 2 gr. dt.

Georius Layminger 2 gr. dt.

Stephanus Wyrsing 2 gr. dt.

Erasmus de Valle Eni 2 gr. dt.

10 Mag. Heinricus de Valle Eni 2 gr. dt.

Nicolaus plebanus in Walthuden 2 gr. dt.

Johannes archidyaconus de Cibinio 2 gr. dt.

Johannes de Segswar 2 gr. dt.

Dom. Bartholomeus in Altzuna 2 gr. dt.

15 Fridericus Kraft 2 gr. dt.

Johannes Wirnt 2 gr. dt.

14 Von s. H. über der Zeile eingetragen.

15 Siehe KINK 2, 136.

16 Davor getilgt „di“. 
Johannes Gwărleich 2 gr.

Johannes Seld 2 gr.

fol. $3^{\text {ra }}$

$[1407 \mathrm{I}]$

(1407) Anno Domini Mo CCCC ${ }^{\circ}$ VII $^{\circ}$ Michael de Aczmanspach baccalarius in decretis promotus est in licenciatum sub venerabili viro domino Lienhardo Schawr decretorum doctore. ${ }^{17}$ Item solvit ad facultatem 3 fl. ${ }^{18}$

fol. $3^{r b}$

$[1407$ II]

\section{Heinricus Kitzpứhel}

(Ca. 1407 Oktober 13) Anno Domini MCCCCVII' circa festum Colmanni in decanum facultatis iuris assumptus est Heinricus Kitzpúhel decretorum doctor, sub quo intitulati sunt infrascripti:

Primo Leonardus Prost canonicus ecclesie Ratisponensis

Item Conradus Henrici de Hildensem

Item Fridericus Stawthaimer de O̊tinga

Item Heinricus de Müldorf

5 Item Johannes Turndl licenciatus in artibus

Item Leonardus Layminger canonicus ecclesie Pataviensis

fol. $3^{v}$

[1408 I]

\section{Caspar Mayselstain}

(Ca. 1408 April 23) Anno Domini $\mathbf{M}^{\circ} \mathbf{C C C C V I I I}^{19}$ circa festum Georgii in decanum facultatis iuris assumptus est dominus Caspar Mayselstain decretorum doctor, sub quo intitulati sunt infrascripti:

Mag. Johannes Rơchel 2 gr. dt.

Johannes Glökelherr 2 gr. dt.

Mathias Regelshofer 2 gr. dt.

Philippus Speyser 2 gr. dt.

5 Judocus Rot 2 gr. dt.

Mag. Albertus de Byla 2 gr. dt.

Erasmus Reyntaler 2 gr. dt.

17 Der Absatz ab „Anno“ wurde von a. H. schon am Ende von fol. $2^{\text {vb }}$ eingetragen, dann jedoch durch Rasur getilgt.

$18 \mathrm{Ab}$,Item“ mit anderer Tinte.

19 Das vierte „C“ von s. H. über der Zeile eingefügt. 
Johannes Wogawer 2 gr. ${ }^{20} 21$

fol. $4^{r}$

[1408 II]

(Ca. 1408 Oktober 13) Anno Domini millesimo CCCC ${ }^{\circ}$ VIII $^{\circ}$ circa festum Cholomani in decanum facultatis iuris assumptus est dominus Michael Aczmanspach licenciatus in decretis, sub quo intytulati sunt infrascripti:

Georius Melchaymer de Prawnaw 2 gr.

Michahel de Sancto Ypolito canonicus regularis 2 gr. dt.

Hainricus de Lawffen 2 gr. dt.

$[1409$ I]

\section{Johannes Sinderami ${ }^{22}$}

(Ca. 1409 April 23) Anno quadringentesimo nono circa festum Sancti Georii assumptus est in decanum facultatis iuris Johannes Sindrami decretorum doctor, sub quo intitulati sunt infrascripti:

Chunradus de Helmstat 2 gr.

Richardus Schlupff 2 gr.

Hermannus Bürser de Veltkilch

Cristannus de Brunna 2 gr.

5 Vlricus Hawsecker 2 gr.

Heinricus Stawthaymer 2 gr.

Jacobus presbyter de Lanczans dt. ${ }^{23} 2$ gr.

Mag. Petrus de Augusta 2 gr.

Nicolaus Speyser 2 gr.

10 Wencesslaus de Brunna 2 gr. dt.

fol $4^{v}$

[1409 II]

\section{Caspar Mayslstain}

(Ca. 1409 Oktober 13) Anno Domini millesimo quadringentesimo nono circa festum Kolmanni in decanum facultatis iuris assumptus est dominus Caspar Mayslstain decretorum doctor, sub quo intitulati sunt infrascripti:

20 Gesamter Eintrag von a. H.

21 Danach wurden an dieser Stelle versehentlich die Einträge zum Semester $1410 \mathrm{I}$ begonnen, und zwar von „Anno“ bis „Thomas [de Septemcastris]“, jedoch später wieder getilgt. Vgl. S. 8.

22 In der Vorlage befindet sich diese Zeile am Kopf von fol. $4^{\mathrm{r}}$.

23 Davor überstrichene Rasur. 
Nicolaus de Prussia 2 gr. dt.

Petrus Pherlini 2 gr. dt.

Mag. [Johannes] ${ }^{24}$ Măgerl 2 gr. dt.

Mag. Christanus de Septemcastris 2 gr. dt.

5 Nicolaus de Rogettel 2 gr. dt.

Johannes Rex 2 gr. dt.

Mag. Petrus de Arnsdorf 2 gr. dt.

Vincencius Weinstok de Holenburg 2 gr. dt.

Nicolaus plebanus in Rüsdarf 2 gr. dt.

10 Sigismundus plebanus in Wirthelm 2 gr. dt.

Mag. Paulus de Wyenna 2 gr. dt.

Johannes Schilher 2 gr. dt.

$[1410 \mathrm{I}]$

\section{Heinricus Kitzpuhel}

(Ca. 1410 April 23) Anno Domini $\mathbf{M}^{\mathrm{CCCCX}}$ circa festum Georgii in decanum facultatis iuris assumptus est Heinricus Kitzpuhel decretorum doctor, sub quo intitulati sunt infrascripti:

Thomas Liechtenuelder 2 gr.

Thomas de Septemcastris 2 gr.

Johannes de Marichpurck 2 gr.

Johannes Velbèr 2 gr.

5 Bartholomeus Kren de Erdpurg 2 gr.

Mag. Nicolaus Krotendarffer 2 gr.

Narcissus ${ }^{25}$ Hercz 2 gr.

Mag. Theodricus Lobing de Erdfordia 2 gr.

fol. $5^{r}$

Johannes Wolflein plebanus in Lind 2 gr.

10 Conradus Eberhardi de Hallis 2 gr.

Nicolaus Krêt 2 gr.

Johannes Amselår 2 gr.

Johannes Sweydnitz canonicus ecclesie Sancte Crucis in Bradislauia 2 gr.

Nicolaus Mathie Goldperg de Wratislauia 2 gr.

15 Mag. Johannes de Voburg 2 gr.

Herdwicus Hawnsperger 2 gr.

Mag. Johannes Butczpach 2 gr.

Dom. Mathias Regelshoffer lic. $1 \mathrm{fl}$.

24 Der fehlende Vorname ist durch zwei Punkte markiert. Zur Emendation Vgl. Uiblein, AFA I, 533: „Johannes Magerl de Septemcastris“.

25 Vorlage: „Narciscus“. 
Stephanus Grêtzer bacc. 1 fl.

[1410 II]

Michael de Aczmanspach licenciatus

(Ca. 1410 Oktober 13) Anno Domini millesimo quadringentesimo $\mathbf{X}^{\mathrm{mo}}$ circa festum Sancti Colomanni in decanum facultatis iuris assumptus est Michael de Aczmanspach licenciatus in decretis, sub quo intitulati sunt infrascripti:

Franciscus Granatoris de O̊ttingen 2 gr.

Chonradus Zeydlarêr de Monaco 2 gr.

Item Gundakerus Aspekch bacc. in decretis $1 \mathrm{fl}$.

Item Martinus Back de Gamundia 2 gr.

5 Item Johannes de Nydden 2 gr.

fol. $5^{v}$

$[1411 \mathrm{I}]$

Magister Gerhardus Vischpekch doctor decretorum canonicus ecclesie Sancti Stephani Wiennensis

(1411 April 14) Anno Domini millesimo quadringentesimo XIo in die Sanctorum Tyburcii et Valeriani in decanum facultatis assumptus est magister Gerhardus Vischpekch decretorum doctor, sub quo intitulati sunt infrascripti:

Item Michel Jeskonis de Resinkirche 2 gr.

Johannes Gnechowicz de Wratislauia 2 gr.

Thomas Matzseêr decanus Maticensis $80 \mathrm{~d}$.

Mathias Cordobok de Gora 2 gr.

5 Nicolaus Wolff de Wartemberg 2 gr.

Johannes Wolf de Wartemberg 2 gr.

Nicolaus Sartoris de Gora p.

Mag. Johannes Hymel de Weytz 2 gr.

Mag. Theodricus de Brunswyg 2 gr.

10 Chunradus Wirsung de Lantzhuta 2 gr.

Johannes Tagersheym 2 gr.

Martinus Ro̊chel 2 gr.

Dom. Petrus Teschner de Wratislauia 2 gr.

Nycolaus Graudencz 2 gr.

15 Nicolaus Sartoris p.

Johannes Westualus de Suzato dr. decretorum assumptus est ad facultatem et promisit solvere etc.

Johannes de Altemburg p. 
Magister Johannes Sinderami decretorum doctor etc.

(Nach 1411 Oktober 13) Anno Domini millesimo quadringentesimo undecimo post festum Sancti Cholomanni in decanum facultatis assumptus est magister Johannes Sinderami decretorum doctor, sub quo intitulati sunt infrascripti:

Vlricus Horlactawer de Monaco plebanus in Holczhausen 2 gr.

Conradus de Salczburga monachus monasterii in Altach 2 gr.

Johannes Traboldi de Bruxella 2 gr.

Johannes Husel p.

5 Nicolaus de Seydlicz plebanus in Nouoforo etc. 2 gr.

Johannes Múyther p.

Rudolfus Bellazon scolasticus et canonicus Curcensis 2 gr.

Fridericus Schenkch de Lymburg Herbipolensis, Bambergensis etc. ecclesiarum canonicus dedit medium flor. videlicet octuaginta $\mathrm{d}$.

Johannes Fưssil de Bratislauia 2 gr.

10 Johannes Tornaw de Lutko 2 gr.

Kaspar Herr de Vlma 2 gr.

Siluester Flieger de Augusta 2 gr.

Johannes Grünbald canonicus ecclesie Frisingensis $80 \mathrm{~d}$.

Otto Seinreich p.

15 Clemens de Agria 2 gr.

Thomas de Septemcastris plebanus in Muschna 2 gr.

Nicolaus Kyczka plebanus in Pleusnicz 2 gr.

Nicolaus prepositus in Brixna $80 \mathrm{~d}$.

Dom. Gotfridus Harscher decanus ecclesie Augustensis $80 \mathrm{~d}$.

20 Conradus Ruyhing de Freyburg 2 gr.

fol. $\sigma^{v}$

[1412 I]

Magister Johannes Schallerman de Westualia decretorum doctor

(1412 April 14) Anno Domini millesimo quadringentesimo XII' in die Sanctorum Tyburcii et Valeriani in decanum facultatis assumptus est magister Johannes de Westualia decretorum doctor, sub quo intytulati sunt infrascripti:

Item Jacobus de Sancto Zenone dt. 2 gr.

Item Philippus de Smolna dt. 2 gr.

Item Nicolaus Vogel de Wratislauia dt. 2 gr.

Item Johannes Go̊dke de Danczk dt. 2 gr.

5 Item Cristannus Murher de Grassertal dt. 2 gr. 
Item Conradus Phòrtner de Jukarim dt. 2 gr.

$\left.\begin{array}{l}\text { Item Reynhardus de Helmstat canonicus ecclesie Spirensis } \\ \text { Item Mag. Petrus Crombach de Pruszella isti duo dederunt }\end{array}\right\} 80 \mathrm{~d} \cdot{ }^{26}$

Item Jacobus de Gilgenburg dt. 2 gr.

10 Item Martinus Petirbicz mansionarius ecclesie Wratislauiensis dt. 2 gr.

Item Petrus de Hëdersdorff dt. 2 gr.

Item Erasmus Kemerèr dt. 2 gr.

Item Johannes Albrand de Bruchsella dt. 2 gr.

Item Petrus de Valle Sancte Marie dt. 2 gr.

15 Item Johannes Schenkchenberger canonicus ecclesie Brixinensis dt. $79 \mathrm{~d}$.

Item Johannes Gawen de Lenicz dt. 2 gr.

Item Michel de Herbelstat dt. 50 d.

Item Mag. Waltherus Scho̊rling canonicus Sancti Germani dt. 2 gr.

Item Rudolfus Westersteter canonicus Augustensis dt. 4 gr.

20 Item Cristannus Wurm de Salina dt. 2 gr.

Item Georius prepositus ecclesie Sepusiensis dt. 12 gr.

Item Bernhardus de Raczenhausen canonicus Basiliensis dt. 2 gr.

Item Johannes Hess de Ysennach ${ }^{27}$ dt. 2 gr.

Item Georius Liechtenberg canonicus ecclesie Beate Marie Glogouie Maioris 2 gr.

25 Item Johannes Jonsdorf clericus Bratislauiensis diocesis dt. 2 gr.

fol. $7^{r}$

[1412 II]

Mag. Petrus Dekinger de Wynna licenciatus in decretis.

(1412 Oktober 13) Anno Domini millesimo quadringentesimo duodecimo in die Sancti Colomanni martyris in decanum facultatis iuristarum assumptus est magister Petrus Dekinger licenciatus in decretis, sub quo intitulati sunt subscripti:

Gotfridus de Oyta 2 gr.

Martinus de Oyta 2 gr.

Mag. Krafto de Swarczach 3 gr.

Johannes Sigel ordinis canonicorum regularium de Walsee 2 gr.

5 Gregorius Ledenter canonicus ecclesie Novi Monasterii Herbipolensis 2 gr.

Andreas archidiaconus et canonicus Olomucensis 2 gr.

Johannes Nicolai de Brunna p.

Frater Heinricus de Inferiori Altach ordinis Sancti Benedicti 2 gr.

Nicolaus Reiberstorffer de Montibus Chutnis 2 gr.

10 Eglolfus Etterli de Bringk in Ergaw 2 gr.

Johannes Spèt de Papenheim 2 gr.

Johannes Woluisheim de Waldbrunn 2 gr.

26 „80 d.“von s. H. am rechten Rand geschrieben und mittels Zuweisungsstrichen diesem und dem vorhergehenden Eintrag zugeordnet.

27 Radiertes „E“ vor „Y“. 
Andreas Nas canonicus Wissegradensis 6 gr.

Dom. Sigismundus de Septemcastris plebanus in Wirthelm pro baccalariatu dt. $1 \mathrm{fl}$.

15 Wilhelmus Kircher bacc. decretorum dt. 1 fl. et $3 \mathrm{gr}$.

Mag. Wolfgangus de Weissenkirchen 3 gr.

Demetrius de Czerod 2 gr. $^{28}$

Johannes Stark plebanus in Aldenpolan 2 gr.

Mag. Martinus de Chranach 2 gr.

20 Conradus $^{29}$ Scharb de Lawben 2 gr.

Zegotha archidiaconus de Borswa et canonicus Agriensis $80 \mathrm{~d}$.

fol. $7 v$

$[1413 \mathrm{I}]$

Magister Wilhelmus Kircher de Constancia doctor decretorum.

(1413 April 14) Anno Domini $\mathbf{M}^{\circ}$ CCCC $^{\circ}$ XIII $^{\mathrm{mo}}$ in die Sanctorum Tiburcii et Valeriani in decanum facultatis assumptus est magister Wilhelmus Kircher de Constancia decretorum doctor, sub quo sunt intytulati infrascripti:

Item Petrus Hecht de Kůnigsperg dt. 2 gr.

Item Mag. Nicolaus canonicus ecclesie Transsiluane et plebanus in Tartala 8 gr.

Item Symon Alberti altarista ecclesie Olmoncensis 3 gr.

Item dom. Symon prepositus Cibiniensis de Rozgon dt. $80 \mathrm{~d}$.

5 Item Andreas de Falkenberg plebanus in Wiselburg 2 gr.

Item dom. Wilhelmus de Hurnhain canonicus ecclesie Augustensis ${ }^{30}$

Item dom. Andreas de Astershofen plebanus in Smaichingen

Item Fridericus Warnhofer de Astauia $80 \mathrm{~d}$.

Item Johannes Felber de Hallis 2 gr.

10 Item Heinricus Deringaw ${ }^{31} 2$ gr.

Item dom. Stephanus plebanus in Falkenstain $80 \mathrm{~d}$.

Item Mag. Johannes Tagerhaim 2 gr.

Item Johannes Zepek de Laybaico 2 gr.

Item Petrus Etter lic. in artibus 2 gr.

15 Item dom. Heinricus de Helmstat prepositus Spirensis $80 \mathrm{~d}$.

Item Mag. Hermannus Haidelshaim 2 gr.

Item Nicolaus Stok de Glogouia Maiori 2 gr.

Item Wilhelmus Pressater de Amberg 2 gr.

Item dom. Michael Rosenkirch bacc. in decretis $1 \mathrm{fl}$.

20 Item Jacobus de Weytra p.

28 Tintenwechsel.

29 Tintenwechsel, davor eine Zeile freigelassen.

30 „80 d.“ mittels Zuweisungsstrichen den Einträgen 6-8 zugeordnet.

31 Korrigiert aus „Doringaw“. 
$\left.\begin{array}{l}\text { Item frater Georius } \\ \text { Frater Conradus }\end{array}\right\}$ de Varembach 6 gr.

Item Conradus Schürg de Tranbach p.

Item dom. Johannes Turinge de Prawnsperg plebanus in Osterror 2 gr.

25 Item dom. Johannes Zimischem archidiaconus de Pankata canonicus Agriensis 6 gr. Item Mag. Pilgrimus de Zegenberg 2 gr.

Item Mag. Petrus Tegerpek de Scheyrn 2 gr.

Item Johannes Swerger de Sulcz 2 gr.

Item dom. Thomas de Muschen bacc. in decretis $1 \mathrm{fl}$.

30 Item Nicolaus Kitschka plebanus in Pleusnicz bacc. decretorum $1 \mathrm{fl}$.

$\left.\begin{array}{l}\text { Item dom. Georius }{ }^{33} \\ \text { Item dom. Conradus }\end{array}\right\}$ de Poppenhaim marscalci $1 \mathrm{fl}$. Rh.

Item Nicolaus amicus decani Patauiensis, canonicus ecclesie Wratislauiensis 2 gr.

fol. $8^{r}$

[1413 II]

\section{Co̊nradus Seglau[er]}

(1413 Oktober 13) Anno Domini M० CCCC XIII' in die Sancti Colmanni in decanum facultatis assumptus est magister Cůnradus Seglauer. In huius decanatu infrascripti sunt intytulati:

Johannes Scriptoris de Frankfordia 2 gr.

Item Mag. Thomas Lurczer $2 \mathrm{gr}$.

Frater Gregorius de Monte Sancti Georii Vallis Eni 2 gr.

Dom. Johannes Winterburg 2 gr.

5 Dom. Antonius Füchtensteiner 3 gr.

Michael Regelzhofer 2 gr.

Nicolaus de Průmea 2 gr.

Tilmannus Joel de Lyris 2 gr.

Mag. Hainricus Tandorfer $4 \mathrm{gr}$.

10 Gothardus de Altach Inferiori 2 gr.

Nicolaus Ysenhütel de Wienna 2 gr.

Mag. Vrbanus de Melk 2 gr.

Dom. Johannes Zink ${ }^{34}$

Johannes Redler famulus eius $\} 84$ [d.]

15 Johannes Cristoferi de Winczig 2 gr.

Vlricus Werder de Papenheim 2 gr.

Thomas Dórndel de Inferiori Altach 2 gr.

Mag. Nicolaus Salczmesser de Frankfordia circa Oderam 2 gr.

32 „de Varembach 6 gr. “ mittels Zuweisungsstrich den Einträgen 21-22 zugeordnet.

33 „de Poppenheim marscalci 1 fl. Rhen." mittels Zuweisungsstrich den Einrägen 31-32 zugeordnet.

34 „84 [d.]“ mittels Zuweisungsstrich den Einträgen 13-14 zugeordnet. 
fol. $8^{v}$

$[1414 \mathrm{I}]$

Dominus Caspar Mayselstain decretorum doctor.

(1414 April 14) Anno Domini millesimo quadringentesimo quartodecimo in die Sanctorum Tyburcii et Valeriani in decanum facultatis assumptus est venerabilis vir dominus Caspar Mayselstain decretorum doctor, sub quo intytulati sunt infrascripti:

$\left.\begin{array}{l}\text { Dom. Sigismundus Flemming de Lantzhuta } 2 \text { gr. } \\ \text { Dom. Phylippus de Cronemberg canonicus Maguntinensis et Treverensis }{ }^{35}\end{array}\right\} 14$ gr.

Franco de Cronemberg

Michael de Lechling 2 gr.

5 Heinricus de Oyta 2 gr.

Wolfgangus Wurm de Freynstat 2 gr.

Dom. Nicolaus de Waradino 2 gr.

Mag. Johannes de Corona 2 gr.

Georius de Zůmelsperg plebanus in Grifen 2 gr.

10 Dom. Albertus de Borzislauicze Cracouiensis et Bosnonensis canonicus 1/2 lb. d.

Thomas Braxatoris de Amsteten 2 gr.

Dom. Johannes Vetter de Memmingen 3 gr.

Item Henningus Lủbersdorp 3 gr.

Heinricus Wlpis de Syndring p.

15 Mag. Heinricus Stoll de Hamelburg 2 gr.

Dom. Paulus de Sana abbas de Bata $\left.^{36}\right\}_{1} \mathrm{fl}$

Frater Georius cappelanus suus

Dom. Leonhardus de Voytzperg 2 gr.

Mag. Petrus Barna 2 gr.

20 Franciscus Rossel 2 gr.

Wilhelmus Köllner 2 gr.

Henningus de Halberstat 2 gr.

Dom. Jacobus de Sancto Vito plebanus in Gnềs 2 gr.

Dom. Dyonisius Jax custos et canonicus ecclesie Transsiluane $12 \mathrm{gr}$.

25 Item Mag. Bernhardus Nebraw 2 gr.

Item Dauid Jung 2 gr.

Item dom. Fredericus Prenner 3 gr.

Idem dom. Fredericus pro baccalariatu $1 \mathrm{fl}$.

Dom. Rudolfus Bellazon canonicus Curiensis pro baccalariatu $1 \mathrm{fl}$.

30 Mag. Johannes de Tagershaim lic. in decretis $4 \mathrm{fl}$.

Mag. Conradus de Hildesheim lic. in decretis $4 \mathrm{fl}$.

35 „14 gr.“ mittels Zuweisungsstrich den Einträgen 2-3 zugeordnet.

36 „1 fl.“ mittels Zuweisungsstrich den Einträgen 16-17 zugeordnet. 
fol. $9^{r}$

[1414 II]

Dominus Michel de Aczmanspach licenciatus in decretis

(1414 Oktober 13) Anno Domini millesimo quadringentesimo quartodecimo in die Sancti Colomanni martyris in decanum facultatis assumptus est venerabilis vir dominus Michael de Aczmanspach licenciatus in decretis, sub quo intitulati sunt infrascripti:

Johannes Folczian de Wienna 2 gr.

Dom. Demetrius de Gich canonicus ecclesie Herbipolensis 12 gr.

Mag. Nicolaus Kơschel de Clawsemburg 2 gr.

Leonhardus Selgerêt de Mồtnicz 2 gr.

5 Dom. Erhardus Satelboger canonicus ecclesie Ratisponensis ${ }^{37}$

Johannes de Herremberg

Mag. Johannes Kirchen iunior

Petrus Schenkch de Weybstat

Item Wenczeslaus Syndel canonicus ecclesie Sancti Petri in Brunna 2 gr.

10 Mag. Conradus de Halstat 3 gr.

Fredericus de Salczpurga 2 gr.

Dom. Heinricus Roll plebanus in Carlowicz necnon canonicus Chremsirensis 12 gr. Johannes Augustini de Ratispona 2 gr.

Mag. Nicolaus Andree de Strellen 2 gr.

15 Gregorius de Landestrost 2 gr.

Item dom. Fredericus Prenner de licencia 2 lb. d..$^{38}$

Item ${ }^{39}$ Mag. Jacobus de Baden tenetur dom. Johannes doctor pro eo 3 gulden

fol. $9^{v}$

Magister Gerhardus Vischpekch doctor decretorum canonicus ecclesie Sancti Stephani Wiennensis.

(1415 April 14) Anno Domini $\mathbf{M}^{\circ} \mathrm{CCCC}^{\mathrm{mo}}$ quintodecimo in die Sanctorum Tiburcii et Valeriani in decanum facultatis assumptus est magister Gerhardus Vischpekch decretorum doctor, sub quo sunt intitulati infrascripti:

Dom. Paulus archidiaconus Symigiensis et canonicus Wesprimiensis 3 sol.

Heinricus Gastmeyster de Gobyn 2 gr.

Johannes de Seccouia 2 gr.

37 „10 gr.“ mittels Zuweisungsstrich den Einträgen 5-8 zugeordnet.

38 „De licencia 2 lb. d.“ von a. H. statt der durchgestrichenen Angabe „3 gulden“.

39 Am linken Seitenrand ,tenetur“. 
Mag. Thomas de Zakan 3 gr.

5 Dom. Theodorus de Gich canonicus et archidyaconus Herbipolensis 12 gr.

Laurencius Kasla de Wratislauia 2 gr.

Dom. Petrus de Heltaw 2 gr.

Clemens Almår de Salzpurga 2 gr.

Johannes Gerhardi de Eysenhusen 2 gr.

10 Erasmus Drüsselchind plebanus in Moltzpüchel 2 gr.

Dom. Johannes Scholym canonicus ecclesie kathedralis Wratislauiensis 8 gr.

Eberhardus Molitoris de Nuremberga p.

Gotfridus Fryling de Oyta bacc. $1 \mathrm{fl}$.

Mag. Krafto de Swarczach dr. decretorum 3 fl. pro licencia ${ }^{40}$

15 Arnoldus de Lawtershaym plebanus ibidem 2 gr.

Johannes de Lapide plebanus in Folxhaim 2 gr.

Dom. Georius Mưrlein plebanus in O̊czing $20 \mathrm{~d}$.

Wilhelmus Reantel de Awsse 2 gr.

Johannes Brisach de Constancia 3 gr.

20 Georius de Rudlinga 2 gr.

$\left.\begin{array}{l}\text { Petrus Sesselman } \\ \text { Bertoldus Sesselman }\end{array}\right\}$ fratres $\left\{\begin{array}{l}4 \text { gr. } \\ 4 \text { gr. }\end{array}\right.$

fol. $10^{r}$

[1415 II]

Magister Johannes Sinderami decretorum doctor.

(1415 Oktober 13) Anno Domini millesimo quadringentesimo quintodecimo in die Sancti Kolomanni martyris in decanum facultatis assumptus est venerabilis vir magister Johannes Sinderami decretorum doctor, sub quo intitulati sunt infrascripti:

Andreas Chlosner de Geren 5 gr.

Johannes Gebhardi plebanus in Enczestorf Maiori 2 gr.

Nicolaus Atyser de Villa Las 2 gr.

Petrus Amsteg de Selgenstad 2 gr.

5 Dom. Johannes Brucenus baccalariandus unum fl. dt.

Frater Vlricus Nernshaymer de Haydenhaym 2 gr.

Mag. Vlricus Czand de Weyssemburga 2 gr.

Johannes Ludberti canonicus Boldmensis 2 gr.

Dom. Georius de Schawmberg canonicus Bambergensis et $14 \mathrm{gr}$.

10 Henricus de Bamberga familiaris eiusdem $\} 14$ gr.

Bertoldus de Helta lic. in artibus 2 gr.

Siluester bacc. dt. unum fl.

Georius de Battaw canonicus Patauiensis dt. 84 d.

cum famulo suo Martino Hublinger de Wels

40 „Pro licencia“ von a. H.

41 „Fratres“ diesem und dem folgenden Eintrag mittels Zuweisungsstrich zugeordnet. 
fol. $10^{v}$

[1416 I]

\section{Magister Caspar Mayselstain decretorum doctor.}

(1416 April 14) Anno Domini millesimo CCCC $^{\text {mo }} \mathbf{X V I}^{\circ}$ in die Sanctorum Tyburcii et Valeriani in decanum facultatis assumptus est magister Caspar Mayselstain decretorum doctor, sub quo sunt intitulati infrascripti:

Dom. Hermannus episcopus Frisingensis ${ }^{42}$

Item dom. de Waldegk canonicus et custos ecclesie Frisingensis $\} 1 \mathrm{fl}$.

Johannes Vbelman de Lantzhuta

Item dom. Petrus Stang de Ersperg 2 gr.

5 Jacobus Pistoris de Nissa 2 gr.

Johannes Preylant de Nissa 2 gr.

Johannes Preym de Cliuis 2 gr.

Georius Vischlein 2 gr.

Vincencius Waller 2 gr.

10 Johannes Poueda 2 gr.

Petrus de Caldario de Lynss 2 gr.

Gregorius de Cosula vicarius ecclesie Bratislauiensis 2 gr.

Andreas Burmeyster de Gribiswaldis 2 gr.

Siluester Wager de Patauia 2 gr.

15 Mag. Jacobus de Inferiori Stokchstal 2 gr.

Johannes Ruschlini alias Judicis de Gewicz 2 gr.

$\left.\begin{array}{l}\text { Marcus Enenkel de Albrechtzperg canonicus Patauiensis } \\ \text { Johannes Harster de Lantzhuta familiaris eius }\end{array}\right\} 12$ gr.

Jodocus Quedenow de Künigsperg 2 gr.

20 Mag. Petrus Pauli de Alba Regali 6 gr.

Mag. Nicolaus clericus de Adorian 2 gr.

Gregorius Grunt de Strigonia 2 gr.

Caspar Fridwalt de Nissa 2 gr.

Johannes Awer canonicus Ratisponenesis 6 gr.

25 Mathias Pernicz de Maydburg 2 gr.

Johannes Tabernatoris 2 gr.

Perchtramus de Veltheim 2 gr.

fol. $11^{r}$

Item dom. Johannes Füszel de Wratislauia pro baccalariatu $1 \mathrm{fl}$.

Item Petrus Hecht de Königsperg pro baccalariatu $1 \mathrm{fl}$.

30 Item dom. Conradus Re̊whing pro baccalariatu $1 \mathrm{fl}$.

Item mag. Petrus Warnaw de Prusia ${ }^{43}$ pro baccalariatu $1 \mathrm{fl}$.

42 „1 fl. “ diesem und den nächsten beiden Einträgen mittels Zuweisungsstrich zugeordnet.

43 „P“ irrig mit pro-Schlinge. 
Item dom. Franciscus Resel de Prusia pro baccalariatu $1 \mathrm{fl}$.

$[1416$ II $]$

Dominus Johannes de Westfalia doctor decretorum.

(1416 Oktober 13) Anno Domini millesimo quadringentesimo XVIo in die Sancti Colomanni martyris in decanum facultatis assumptus est magister Johannes de Westfalia decretorum doctor, sub quo sunt intitulati subscripti:

Item Johannes Sompol de Thodia 2 gr.

Item Wolfgangus plebanus in Hartperg 4 gr.

Hermannus de Cassela 2 gr.

Andreas canonicus de Sancto Yppolito 2 gr.

5 Item Johannes de Olmuncz 2 gr.

Item Johannes Finsterwald(er $)^{44}$ de Zitauia 2 gr.

Nicolaus Ecker 2 gr. $^{45}$

Math[ia]s Stephani de Soldino 2 gr.

Fridericus Grenn de Rotemberga 2 gr.

10 Mag. Andreas Mawser 2 gr. $^{46}$

Marquardus de Messenpach canonicus Patauiensis 6 gr.

Petrus Chotter de Patauia 2 gr.

Wenczeslaus Sewberleich de Deschna 2 gr.

Dom. Theodricus de Staffel canonicus Maguntinus 12 gr.

15 Item dom. Petrus Henckel 2 gr.

Bartholomeus Glawbis 2 gr.

Martinus Wanislant de Rosenheim 2 gr.

Jeronimus de Påmburg 2 gr.

fol. $11^{v}$

Item dom. Nicolaus de Chasma 3 gr.

20 Conradus Schedlein 2 gr.

Thomas de Coritnicza 2 gr.

Andreas de Wittemberg 2 gr.

Mag. Johannes Holczman de Aw 2 gr.

Item Mag. Vrbanus de Emerstorf 2 gr.

25 Conradus Seczel de Rütlinga 3 gr.

Dom. Jacobus Bawdewek plebanus in Nissa 4 gr.

Johannes Newsteter canonicus in Anspach $18 \mathrm{~d}$.

Mag. Heinricus Rosemberg canonicus ecclesie Waciensis 3 gr.

Dom. Martinus Helman plebanus in Monte Petri 2 gr.

44 Die Endung „-er“ unsichere Lesung.

45 „2 gr." sowohl unmittelbar nach dem Namen als auch rechts außen eingetragen.

46 Nach dem Namen durchgestrichen „2“. 
30 Mag. Nicolaus de Helta 2 gr.

Johannes Erbeiter canonicus ecclesie Sancti Johannis in Hawg 2 gr.

Liebhardus de Ratispona plebanus in Frêtinga 2 gr.

Item Martinus de Ratispona 2 gr.

Chonradus Schober de Hasfordia 2 gr.

35 Item dom. Conradus Rühing lic. $3 \mathrm{fl}$.

Item Mag. Petrus Warnaw lic. 3 fl.

Item dom. Franciscus Resil lic. $3 \mathrm{fl}$.

Item Nicolaus Goltperg de Wratislauia bacc. $1 \mathrm{fl}$.

Item Wilhelmus Kolner de Danczk bacc. $1 \mathrm{fl}$.

40 Item Mag. Petrus Warnaw dr. $1 \mathrm{fl}$.

Item dom. Franciscus Resil dr. $1 \mathrm{fl}$.

fol. $12^{r}$

$[1417$ I]

Conradus Råwhing licenciatus in decretis.

(1417 April 14) Anno Domini millesimo etc. XVII in die Sanctorum Tiburcii et Valeriani martyrum in decanum facultatis assumptus est dominus Conradus $R^{a}$ ewching licenciatus in decretis, sub quo intitulati sunt infrascripti:

Item Johannes de Manderscheyt ${ }^{47}$ canonicus ecclesie Treuerensis dt. 1/2 fl. ${ }^{48}$

Petrus Hoszil canonicus ecclesie collegiate in Anolspach 3 gr.

Briccius de Buda 2 gr.

Erasmus de Lantzhuta 2 gr.

5 Andreas de Sittauia 2 gr.

Andreas Pfaffendorff canonicus ecclesie Sambiensis Ordinis Tewtonicorum 1/2 fl.

Bernhardus de Sindolfing 2 gr.

Dom. Heinricus Gastmeister de Gobin bacc. $1 \mathrm{fl}$.

Mathias Bernicz Saxo bacc. $1 \mathrm{fl}$.

10 Frater Wolfgangus de Admont 4 gr.

Johannes Pleyntinger 2 gr.

Frater Johannes professus monasterii Jerucensis Ordinis Premonstratensis 4 gr.

Johannes de Znoyma altarista 2 gr.

Heinricus Eglinger de Waibling 2 gr.

15 Bruno Trudelonis de Ytstein 2 gr.

Ambrosius Neczowencz de Golaw 2 gr.

Heinricus Rawch de Tagersheim 2 gr.

Wilhelmus de Hürnhayn canonicus Augustensis 1/2 fl.

Petrus Polcz plebanus in Marăusch 2 gr.

20 Mag. Heinricus Copericz de Budesheim 2 gr.

47 Korrigiert aus "Mandelscheyt“.

48 Darüber von einer Hand des 16. Jh.: „sunt comites“. 
fol. $12^{v}$

[1417 II]

Magister Gerhardus Vischpekch doctor decretorum, canonicus ecclesie Sancti Stephani.

(1417 Oktober 13) Anno Domini millesimo quadringentesimo XVII in die Sancti Cholomanni martyris in decanum facultatis assumptus est magister Gerhardus Vischpekk decretorum doctor, sub quo sunt intytulati infrascripti:

Baccalarii:

Item Mag. Heinricus Tandorffer de Nurmberga $1 \mathrm{fl}$.

Item dom. Nicolaus de Waradino de Ungaria $1 \mathrm{fl}$.

Item dom. Paulus abbas de Batha $2 \mathrm{fl}$.

Item mag. Johannes Merswein de Argentina $1 \mathrm{fl}$.

5 Item Philippus Dino de Smolna $1 \mathrm{fl}$.

Item dom. Fridricus Kraft pro baccalariatu $1 \mathrm{fl}$.

Item idem dom. Fridricus Kraft pro licencia $3 \mathrm{fl}$.

Supposita intitulata:

Item mag. Johannes Prunger de Frankfordia bacc. 3 gr.

Item mag. Hermannus Wulco de Frankenfordis 2 gr.

10 Item Johannes Amelprecht de Nicolspurga 2 gr.

Item Hodiko plebanus in Radhost 2 gr.

Item mag. Jacobus Angeli de Vlma 3 gr.

Item dom. Michael Falkonis arcium mag., decretorum bacc. et dr. in medicina 6 gr.

Item Hermannus Deys beneficiatus in ecclesia Paderburnensis 2 gr.

fol. $13^{r}$

$[1418 \mathrm{I}]$

Dominus Fridricus Krafft decretorum doctor, canonicus ecclesie Patauiensis necnon plebanus in Falkenstayn.

(1418 April 14) Anno Domini millesimo CCCC' XVIII in die Sanctorum Tyburcii et Valeriani martyrum in decanum facultatis assumptus est dominus Fridricus Krafft decretorum doctor, sub quo sunt intytulati infrascripti:

Item Nicolaus Wyel de Wlessna 2 gr.

Item mag. Johannes de Frankfordia bacc. in decretis pro admissione ad repetitionem facultatis $1 \mathrm{fl}$.

Item dom. Fridricus Krafft pro doctoratu $1 \mathrm{fl}$.

Item dom. Vincencius de Jordansmúl canonicus Wratislauiensis 12 gr. 
5 Item Johannes de Måyrzz 2 gr.

Item dom. Nicolaus Johannis de Frigido Fonte 2 gr.

Item mag. Benedictus de Worach 3 gr.

Item mag. Nicolaus de Themezwar 3 gr.

Item mag. Barnabas Tyzzawazzan 3 gr.

10 Item Erkengerus de Sawnsheim canonicus Herbipolensis $83 \mathrm{~d}$.

Item Andreas Putnyt de Aw 3 gr.

Item Wolfgangus Kerspek canonicus ad Sanctum Florianum ${ }^{49}$

Item Johannes Loman de Riga famulus

Item Johannes Godke de Wratislauia

15 Item Johannes Fyol de Toran

Item dom. Rudolfus decanus ecclesie Sancti Petri Basiliensis 12 gr.

Item dom. Nicolaus Zedlicz canonicus ecclesie Bregensis 12 gr.

Item Laurencius de Rota plebanus in Kirchdorff 2 gr.

Item Oswaldus de Salina 2 gr.

20 Item Johannes Deys 2 gr.

Item Clemens Drzewiczky 2 gr.

Item Mathias de Gnyznă 2 gr.

Item dom. Conradus Seybolt de Memmingen 2 gr.

fol. $13^{v}$

$[1418 \mathrm{II}]$

Magister Petrus Dekchinger licenciatus in decretis canonicus ecclesie Sancti Stephani necnon vicecancellarius studii Wiennensis.

(1418 Oktober 13) Anno Domini millesimo CCCC $^{\text {mo }}$ XVIII $^{\circ}$ in die Sancti Colomani martyris in decanum facultatis assumptus est magister Petrus Dekchinger licenciatus in decretis, sub quo sunt intitulati infrascripti:

Item Leonhardus Meylestorffer 10 gr.

Item Mag. Nicolaus Weinschenk de Rudlinga 2 gr.

Item Johannes de Bybraco 2 gr.

5

Licenciati et baccalarii
Item mag. Johannes Merswein pro licencia dt. 3 fl. ${ }^{50}$ Item mag. Heinricus Tandorffer de Nuremberga pro licencia $3 \mathrm{fl}$.

Item mag. Pilgrimus de Zegenberg pro licencia et baccalariatu $4 \mathrm{fl}$.

Item mag. Vlricus de Weissemburga pro baccalariatu dt. $1 \mathrm{fl}$.

Item Heinricus Scriptoris de Frankfordia pro baccalariatu $1 \mathrm{fl}$.

49 „4 gr.“ mittels Zuweisungsstrich auch den Einträgen 12-15 zugeordnet.

50 „Licenciati et baccalarii“ am linken Seitenrand mittels Zuweisungslinien den Einträgen 4-8 zugeordnet. 
Item $^{51}$ dom. Vlricus comes de Artemberg canonicus ecclesie Patauiensis et Ratisponensis

10 Item mag. Georius Wispeck

Item dom. Wenceslaus de Osterhofen

Item Johannes Tullinger

1 fl. Ung.

Item dom. Johannes de Stukardia prepositus ecclesie collegiate in Boll 10 gr.

Item dom. Ladislaus de Macedonia dt. $88 \mathrm{~d}$.

15 Item Benedictus de Bathor baro 3 sol. $\mathrm{d}$.

Item Michael de Stolczenberg 2 gr.

Item mag. Conradus Schenkch de Külsheim ${ }^{52} 2$ gr.

Item Heinricus Wollemberg de Wismaria 2 gr.

Item Wilhelmus de Weynsberg bacc. arcium 3 gr.

20 Item Erasmus Rubennaw de Gribswaldis 4 gr.

Item Thomas Furenrider de Amberga $18 \mathrm{~d}$.

Item Johannes Wücherl de Lewben p.

fol $14^{r}$

Item dom. Petrus plebanus de Pamenczina 3 gr.

Item Michel Phêfffel de Ingolstat 2 gr.

25 Item Wilhelmus Erhardi de Leydis 2 gr.

Item dom. Michael de Insula Christiana plebanus 6 gr.

Item Stephanus de Zelesna 2 gr.

Item Nicolaus Clementis 2 gr.

Item Wernhardus Kugelrêawter 2 gr.

[1419 I]

Dominus Mathias Regelshouer licenciatus in decretis.

(1419 April 14) Anno Domini millesimo CCCC XIX' in die Sanctorum Tiburcii et Valeriani martyrum in decanum facultatis assumptus [est] dominus Mathias Regelshouer de Herriden licenciatus in decretis, sub quo sunt intitulati infrascripti:

Baccalarii $\left\{\begin{array}{l}\text { Item dom. Wenceslaus Sündel de Brunna pro baccalariatu } 1 \mathrm{fl} .^{53} \\ \text { Item Georius Vischel pro baccalariatu } 1 \mathrm{fl} . \\ \text { Item mag. Conradus Halstat pro baccalariatu } 1 \mathrm{fl} .\end{array}\right.$

Item Johannes Yencz de Wratislauia 2 gr.

5 Oswaldus Strawss de Ratispona 2 gr.

Mag. Thomas de Achspach $15 \mathrm{~d}$.

Item dom. Johannes Chlosner canonicus Patauiensis $88 \mathrm{~d}$.

51 Dieser und die folgenden drei Einträge sind von s. H. durch eine Linie am linken Seitenrand als zusammengehörig gekennzeichnet worden.

52 „de Külsheim“v. a. H. hinzugefügt.

53 Am linken Rand durch Klammer diesem und den beiden folgenden Einträgen zugewiesen. 
Item dom. Ladislaus de Marcellis prepositus Chanadiensis ${ }^{54}$

Item Blasius Chanadiensis cappellanus predicti prepositi $\} 1 \mathrm{fl}$.

10 Item Albertus de Kerot familiaris eiusdem prepositi $\}$

Item Bernhardus Lang canonicus Sancti Georii in castro Gnesensi 10 gr.

Item Martinus de Walthawsen canonicus regularis $4 \mathrm{gr}$.

fol. $14^{v}$

Item Vlricus Reysperger canonicus Patauiensis, plebanus ${ }^{55}$ ad Sanctum Nicolaum prope Villacum dt.

Item mag. Johannes Gartner de Perching 4 sol. d.

$\left.\begin{array}{l}15 \text { Item Jacobus Blassemberger de Peraw }{ }^{\mathrm{e}}{ }^{56} \\ \text { Item Heinricus Roter }\end{array}\right\} 16$ gr.

Item mag. Heinricus Tandorffer pro doctoratu $1 \mathrm{fl}$.

$\left.\begin{array}{l}\text { Item mag. Johannes de Sweinfordia } \\ \text { Item Georius frater eius }\end{array}\right\} 4$ gr.

20 Item mag. Fridericus Purger de Helpach 2 gr.

Item mag. Petrus Pachmůlner 2 gr.

Item Nicolaus Gloting 2 gr.

Item Michel Liebhardi plebanus in Waldarn 2 gr.

[1419 II]

\section{Magister Caspar Mayselstain decretorum doctor.}

(1419 Oktober 13) Anno Domini $\mathbf{M}^{\circ} \mathbf{C C C C}^{\circ} \mathbf{X I X}^{\circ}$ in die Sancti Colomanni martyris in decanum facultatis assumptus est magister Caspar Mayselstain decretorum doctor, sub quo sunt intitulati infrascripti:

Item dom. Nicolaus Heroldi de Frawnstat canonicus et scolasticus Glogouiensis Maioris dt. 8 gr.

Item Petrus Absolonis de Herdlof 2 gr.

Item Heinricus Fewchter 8 gr.

Item Nicolaus Wolf de Wartemberg 3 gr.

5 Item Conradus Wolff de Wartemberg frater 3 gr.

Item dom. Andreas canonicus Agriensis 5 gr.

fol. $15^{r}$

Item mag. Paulus Troppawer de Wienna 3 gr.

Item Thomas Mearher de Rastenueld 2 gr.

54 „1 fl. " mittels Zuweisungslinien diesem und den beiden folgenden Einträgen zugeordnet.

55 „4 sol. d.“ mittels Zuweisungslinien diesem und dem folgenden Eintrag zugeordnet.

56 „16 gr.“ mittels Zuweisungslinien diesem und dem folgenden Eintrag zugeordnet.

57 „4 gr.“ mittels Zuweisungslinien diesem und dem folgenden Eintrag zugeordnet. 
Item dom. Georius Institoris de Lobyn 2 gr.

10 Item dom. Nicolaus Micheler 2 gr.

Item dom. Johannes de Hermansdorf canonicus ecclesie Warmiensis 3 sol. d.

$\left.\begin{array}{l}\text { Item dom. Albertus de Potendorff } \\ \text { Item Johannes Hewndel famulus }\end{array}\right\} 1 \mathrm{fl}$.

Item Stephanus Phister de Steyer 2 gr.

Promoti:

15 Item dom. Nicolaus Kitzka pro recepcione ad facultatem $3 \mathrm{fl}$.

Item mag. Johannes de Franckfordia pro doctoratu $1 \mathrm{fl}$.

Item mag. Johannes de Franckfordia pro licencia $3 \mathrm{fl}$.

Item mag. Petrus Deckinger pro doctoratu $1 \mathrm{fl}$.

Item mag. Paulus de Wienna pro baccalariatu $1 \mathrm{fl}$.

20 Item idem mag. Paulus pro licencia $3 \mathrm{fl}$.

Item dom. Johannes Gwerleich pro licencia $3 \mathrm{fl}$.

Item dom. Johannes Seld pro licencia $3 \mathrm{fl}$.

Item dom. Nicolaus de Waradino pro licencia $3 \mathrm{fl}$.

$[1420 \mathrm{I}]$

\section{Dominus Johannes Sindrami decretorum doctor.}

(1420 April 14) Anno Domini millesimo CCCC $^{\mathbf{m o}} \mathbf{X X}^{\circ}$ in die Sanctorum martyrum Tyburcii et Valeriani in decanum facultatis assumptus est dominus Johannes Sinderami decretorum doctor, sub quo sunt intitulati infrascripti:

Item dom. Cristannus Håppel plebanus in Puech 3 gr.

Item Nicolaus Cristanni de Reddin dt. 2 gr.

fol. $15^{v}$

Item mag. Conradus de Wienna 4 gr.

Item dom. Leonardus Gruber plebanus in Sweygkers 3 gr.

5 Item mag. Jodocus Rot de O̊berlinga 3 gr.

Item Johannes Lewbner de Nova Civitate 2 gr.

Laurencius Stephani de Corpona 2 gr.

Item dom. Johannes Ebner de Chremsa 4 gr.

Item dom. Cristofferus Welczer 3 sol. d.

10 Item Nicolaus de Asparn 2 gr.

Item Fabianus de Tardascheydn 2 gr.

Item dom. Heinricus Clodebogk 4 gr.

Item dom. Petrus de Toran nihil propter paupertatem

Item dom. Petrus de Herczogburga 2 gr.

58 „1 fl.“ mittels Zuweisungslinien diesem und dem folgenden Eintrag zugeordnet. 
15 Item frater Conradus professus monasterii Wymbergensis $2 \mathrm{gr}$.

Promoti:

Item dom. Johannes Fưssel pro licencia $3 \mathrm{fl}$.

Item dom. Nicolaus Chasmensis pro baccalariatu $1 \mathrm{fl}$.

Item dom. Johannes Spêt pro baccalariatu $1 \mathrm{fl}$.

Item dom. Vincencius pro baccalariatu $1 \mathrm{fl}$.

20 Item dom. Wilhelmus pro baccalariatu $1 \mathrm{fl}$.

Item dom. decanus Brixinensis pro baccalariatu, licencia et doctoratu $5 \mathrm{fl}$.

[1420 II]

Magister Paulus Páwrl de Wienna decretorum doctor.

(1420 Oktober 13) Anno Domini $\mathbf{M}^{\circ} \mathbf{C C C C}^{\circ} \mathbf{X X}^{\circ}$ in die Sancti Colomanni in decanum facultatis assumptus est magister Paulus Pâwrl de Wienna decretorum doctor, sub quo intitulati sunt infrascripti:

Item mag. Johannes Rêwtemberger plebanus in Tytmaning 3 gr.

Item Fredericus Tifer de Constancia 2 gr. ${ }^{59}$

Item dom. Christoforus de Milaslauia $2 \mathrm{gr}$.

fol. $16^{r}$

$\left.\begin{array}{l}\text { Item Johannes Lamberg de Wratislauia }{ }^{60} \\ \text { Item Mathias de Hermansdorff famulus }\end{array}\right\} 1 / 2 \mathrm{lb} . \mathrm{d}$.

Item frater Vlricus de Mềnsee $3 \mathrm{gr}$.

Item Nicolaus Heappel de Grassaw 2 gr.

Item Cristamus de Reddino 2 gr.

Item mag. Michel Moschicz 2 gr.

10 Item dom. Nicolaus Johannis de Dacia 2 gr.

Item dom. Hugo de Leynsteten abbas Alperspacensis $1 / 2$ tl. d.

Item frater Vitus de monasterio Admontensi 6 gr.

Item dom. Martinus in Habelswerd plebanus et canonicus Sancte Crucis Wratislauiensis $60 \mathrm{~d}$.

Item dom. Petrus de Alba Transsiluana $42 \mathrm{~d}$.

15 Item Johannes de Mawttarn $28 \mathrm{~d}$.

Item Petrus Cerdonis presbyter vicarius in Melsak 2 gr.

Item Paulus Syndrami de Nissa 4 gr.

Item Petrus Nabok de Nissa 2 gr.

Item mag. Leo Geysenuelder de Ratispona 2 gr.

20 Item Virgilius Albner de Salzburga 2 gr.

59 „2 gr." und der folgende Eintrag mit anderer Tinte.

$60,{ }^{1 / 2} \mathrm{lb}$. d." mittels Zuweisungslienien diesem und dem folgenden Eintrag zugeordnet. 
Item dom. Petrus de Sancto Ypolito canonicus regularis ${ }^{61} 2$ gr.

Promoti:

Item idem mag. Pa[u]lus Pàwrl de Wienna decretorum dr. $1 \mathrm{fl}$. Item dom. Johannes Seld de Wienna decretorum dr. $1 \mathrm{fl}$.

Item dom. Johannes Fuessel de Wratislauia decretorum dr. $1 \mathrm{fl}$.

25 Item dom. Johannes de Sitauia de baccalariatu $1 \mathrm{fl}$.

Item dom. Johannes Tornaw de Lücko de baccalariatu $1 \mathrm{fl}$.

Item dom. Johannes de Meyrs bacc. in decretis $1 \mathrm{fl}$.

fol. $16^{v}$

[1421 I]

Dominus Johannes Gwerleich decretorum doctor.

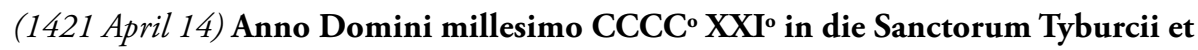
Valeriani martyrum in decanum facultatis assumptus est dominus Johannes Gwerleich decretorum doctor, sub quo sunt intitulati infrascripti:

Item primo dom. ${ }^{62}$ Oswaldus canonicus Salzburgensis, comes de Artenberg dt. $1 \mathrm{fl}$. Item Hertwicus de Zumelsperg ${ }^{63}$ plebanus in Griuen 2 gr.

Item Georius Ekkcherl plebanus in Gurkchueld 3 sol.

Item mag. Wilhelmus Span de Weyssenhorn 3 gr.

5 Item dom. Johannes Gwerleich racione doctoratus $1 \mathrm{fl}$.

Item dom. Vincencius Jordansmül pro licencia $3 \mathrm{fl}$.

Item dom. Wilhelmus de Leydis pro licencia $3 \mathrm{fl}$.

Item Georius Albrechtzperger 2 gr.

Item Wolfgangus Payer de Hederstorff 2 gr.

10 Item dom. Michel rector ecclesie in Napelstorff 4 gr.

Item dom. Johannes de Rosenaw plebanus ad Sanctum Johannem prope Grauenwerd 2 gr.

Item dom. Nicolaus Mayr rector capelle in Weyding 3 gr.

Item dom. Bohoslaus de Lascho 2 gr.

Item mag. Johannes Raczenhawsen plebanus in Wulfesheim 5 gr.

15 Item Leonardus Rastaker de Villaco canonicus Inticensis 2 gr.

Item Henricus Rawch pro baccalariatu $1 \mathrm{fl}$.

Item mag. Nicolaus de Temswar pro baccalariatu $1 \mathrm{fl}$.

Item Fabianus de Tardaschedy pro baccalariatu $1 \mathrm{fl}$.

Item dom. Nicolaus de Waradino pro doctoratu $1 \mathrm{fl}$.

20 Item mag. Nicolaus Stokch de Glogouia pro baccalariatu $1 \mathrm{fl}$.

Item mag. Barnabas de Tysswassan pro baccalariatu $1 \mathrm{fl}$.

61 "Canonicus regularis“ nachträglich mit anderer Feder ergänzt.

62 Vorlage: „d.“, auf Rasur.

63 Danach durch Rasur entstandene Lücke. 
Item dom. Andreas de Wittemberg pro baccalariatu $1 \mathrm{fl}$.

Item Andreas Michaelis de Zerblin 2 gr.

Item dom. Johannes Vey de Wratislauia, altarista ibidem 2 gr.

25 Item Heinricus Nosticz nichil p.

Item dom. Wilhelmus Gerardi de Leydis scolasticus et canonicus Traioctensis pro doctoratu $1 \mathrm{fl}$.

fol. $17^{r}$

[1421 II]

\section{Dominus Johannes de Westfalia decretorum doctor.}

(1421 Oktober 13) Anno Domini millesemo CCCC $^{\text {mo }}$ XXI' in die Sancti Colomanni martyris in decanum facultatis assumptus est dominus Johannes de Westfalia decretorum doctor, sub quo sunt intitulati infrascripti:

Primo dom. Johannes de Perchtoltzdorff canonicus Newmburgensis, decretorum dr. pro recepcione ad facultatem dt. $3 \mathrm{fl}$.

Item Vlricus Werthart de Ratispona $15 \mathrm{~d}$.

Item dom. Conradus Reysperger plebanus in Monte Sancti Viti 3 sol. d.

Item dom. Johannes plebanus in Schenck Maiori 3 gr.

5 Item dom. Jodocus Gesler canonicus Eystetensis 3 gr.

Item dom. Vlricus Lager de Menning rector parochialis ecclesie in Ebelspach 3 gr.

Item frater Symon de Altemburg 4 gr.

Item dom. Mathias Beschorn de Noua Ciuitate 2 gr.

Item dom. Heinricus de Heawen cantor et canonicus Argentinensis 13 gr.

10 Item Oswaldus Holer de Valle Eni 2 gr.

Item dom. Ernestus Mêssemperger plebanus in Grawscharn ${ }^{64}$

Item Vlricus Gewalt de Newmburga

Item Johannes de Gmvnden

Item Tylmannus Helwich ${ }^{65}$ plebanus in Weydembach 3 gr.

15 Item mag. Johannes Würcher de Vlma 3 gr.

Item dom. Wolfgangus de Sancto Floriano pro baccalariatu $1 \mathrm{fl}$.

Item dom. Georius Institoris de Lo̊ben pro baccalariatu $1 \mathrm{fl}$.

Item dom. Heinricus plebanus in Scheybs pro baccalariatu $1 \mathrm{fl}$.

Item Procopius Stampen de Wratislauia 2 gr.

20 Item Heinricus de Napurga p.

fol. $17^{\circ}$

64 "1 fl." mittels Zuweisungslinien diesem und den beiden folgenden Einträgen zugeordnet.

65 Über der Zeile nachgetragen. 
[1422 I]

Dominus Johannes de Perchtoltzdorff canonicus Newmburgensis, decretorum doctor. (1422 April 14) Anno Domini millesimo quadringentesimo XXII' in die Sanctorum Tiburcii et Valeriani martyrum in decanum facultatis assumptus est dominus Johannes de Perchtoltzdorff canonicus Newmburgensis et decretorum doctor, sub quo intitulati sunt infrascripti:

Item dom. Colomannus de Hyppleins canonicus Newmburgensis bacc. in artibus 6 gr. Item mag. Thomas O̊der de Achspach pro baccalariatu $1 \mathrm{fl}$.

Item mag. Petrus Pachmúlner pro baccalariatu $1 \mathrm{fl}$.

Item dom. Nicolaus Speyser plebanus in Meyssilting pro baccalariatu $1 \mathrm{fl}$.

5 Item Jacobus Johannis de Mưsson p.

Item Heinricus Streytperger canonicus Ratisponensis 6 gr.

Item Stephanus Johannis de Grểuendorff 2 gr.

Item mag. Wenczeslaus de Sitauia 2 gr.

Item domini Nicolaus et

10 Conradus Wolf fratres pro baccalariatu $2 \mathrm{fl}$.

Item dom. Cristoferus Lusning plebanus in Strasgang 3 sol. d.

Item Heinricus Engelfrid de Spira 2 gr.

Item Johannes Gnendorffer custos et canonicus Ratisponensis 1/2 lb. d.

Item Martinus de Wolfpach bacc. in artibus 2 gr.

15 Item Jodocus Mểntelberger de Ynsprucka 2 gr.

Item Heinricus de Choburg 2 gr.

Item Johannes de Kyczing 2 gr.

Item dom. Johannes canonicus Jerucensis pro baccalariatu $1 \mathrm{fl}$.

Item mag. Symon de Asparn 2 gr.

20 Item mag. Erhardus Herrant de Newmburga Claustrali 2 gr.

Item dom. Antonius plebanus in Mùllenbach de Septemcastris $4 \mathrm{gr}$.

Item Conradus de Campidena p.

fol. $18^{r}$

Item mag. Conradus de Halstat pro licencia et doctoratu $4 \mathrm{fl}$.

Item dom. Nicolaus canonicus Chasmensis pro licencia et doctoratu $4 \mathrm{fl}$.

25 Item mag. Nicolaus Stock de Maiori Glogouia precentor cripte ecclesie Sancte Crucis Wratislauiensis pro licencia et doctoratu $4 \mathrm{fl}$.

Item mag. Nicolaus de Temeswar pro licencia et doctoratu $4 \mathrm{fl}$.

Item dom. Johannes de Sitauia pro licencia $3 \mathrm{fl}$.

Item mag. Barnabas de Tyssawassan pro licencia et doctoratu $4 \mathrm{fl}$.

Item Michel Heylsmar de Stolczemburg pro baccalariatu $1 \mathrm{fl}$. 


\section{Magister Nicolaus Stokk de Maiori Glogouia decretorum doctor. ${ }^{66}$}

(1422 Oktober 13) Anno Domini millesimo quadringentesimo XXII in die Sancti Colomanni martyris in decanum facultatis assumptus est magister Nicolaus Stok de Maiori Glogouia decretorum doctor, precentor cripte ecclesie Sancte Crucis in Summo Wratislauiensis, sub quo sunt intytulati:

Dom. Nicolaus Gramus Wratislauiensis ac Olomucensis ecclesiarum canonicus 1/2 lb. Dom. Nicolaus Wenke canonicus ecclesie Vratislauiensis, prepositus Othmuchouiensis, bacc. in decretis dt. $1 / 2 \mathrm{lb}$. $\mathrm{d}$.

Item mag. Conradus Langstat 2 gr.

Item Petrus de Heympach bacc. arcium 2 gr.

5 Item Johannes Jewkewicz de Possilwicz 3 gr.

$\left.\begin{array}{l}\text { Item Erasmus Freyberger canonicus ecclesie Salczburgensis }{ }^{67} \\ \text { Item Jacobus Luf de Weyssemburga famulus }\end{array}\right\} 1 / 2 \mathrm{lb}$.

Item mag. Johannes Heresinger de Strawbinga 2 gr.

Item Conradus Reyndel de Fredeberg 2 gr.

10 Item dom. Johannes de Meyrs ad licenciam suam 3 fl. in auro

Item mag. Benedictus de Waradino 2 gr.

Item mag. Stephanus de Ehingen 2 gr.

Item mag. Jacobus Frieshaymer de Schirling 2 gr.

Item Johannes de Semelin de Vngaria 2 gr.

fol. $18^{v}$

$[1423 \mathrm{I}]$

Magister Conradus de Halstat decretorum doctor.

(1423 April 14) Anno Domini Mo quadringentesimo vicesimo tercio in die Sanctorum Tiburcii et Valeriani martyrum in decanum facultatis assumptus est magister Conradus de Halstat decretorum doctor, sub quo sunt intitulati infrascripti:

Item dom. Johannes de Eyb canonicus ecclesie Eystetensis 3 sol. d.

Item Conradus de Elbang 2 gr.

Item Wigandus ${ }^{68}$ Menkler de Hohemberg 2 gr.

Item Nicolaus Kalman de Hoczemplocz 2 gr.

5 Thomas $^{69}$ de Gelesdorff 2 gr.

Dom. Petrus Sculteti de Russia 2 gr.

Dom. Bartholomeus Menseer plebanus in Hymperg $60 \mathrm{~d}$.

66 Die Zeile wurde als Kopfzeile zu Beginn von fol. $18^{\mathrm{r}}$ eingetragen.

$67,1 / 2 \mathrm{lb}$. “ mittels Zuweisungslinie diesem und dem folgenden Eintrag zugeordnet.

68 Von s. H. auf Rasur.

69 Von s. H. auf Rasur. 
Dom. Lodowicus canonicus de Herczogemburg 3 gr.

Dom. Heinricus Burggrauius canonicus ecclesie Augustensis 3 sol. d.

10 Dom. Johannes de Rotemberg ordinis Premonstratensis 2 gr.

Mag. Johannes Lehener de Purchhawsen 2 gr.

Dom. Martinus Czeller de Grăwtzensteten 2 gr.

Dom. Manussius rector ecclesie in Vodwat, bacc. in decretis p.

Valentinus Miskowicz 2 gr.

15 Mathias de Quinqueecclesiis bacc. in artibus 2 gr.

Petrus Hekch de Reyspach bedellus theologie et medicine p.

Dom. Johannes de Leonrod canonicus ecclesie Eystetensis 3 sol. d.

Dom. Cyriacus Knot canonicus ecclesie Eystetensis 3 sol.

Mag. Johannes Ambrosii de Gvnczpurg 2 gr.

20 Johannes de Eych 2 gr.

Johannes Pflancz 2 gr.

Johannes Doleatoris p.

Dom. Bartholomeus Sauigerue prepositus ecclesie kathedralis Tarbatensis $1 \mathrm{fl}$. Vng. Mag. Cristoferus Guldein 2 gr. $^{70}$

fol. $19^{r}$

Promoti in eodem decanatu:

25 Item dom. Wolfgangus Kerspek canonicus regularis Sancti Floriani pro gradu licencie 3 fl. Vng.

Item dom. Johannes Hess de Ysennach pro baccalariatu $1 \mathrm{fl}$. Vng.

Item idem dom. Johannes pro gradu licencie $3 \mathrm{fl}$.

Item dom. Petrus archidiaconus Transsiluanensis pro baccalariatu $1 \mathrm{fl}$.

Item dom. Andreas canonicus Agriensis pro baccalariatu $1 \mathrm{fl}$.

30 Item dom. Petrus Nowag de Nissa pro baccalariatu $1 \mathrm{fl}$.

Item dom. Nicolaus Cluting de Traiecto pro baccalariatu $1 \mathrm{fl}$.

Item dom. Fredericus Reschel plebanus in Vahendorf pro baccalariatu $1 \mathrm{fl}$.

$[1423 \mathrm{II}]$

\section{Dominus Johannes Hess licenciatus. ${ }^{71}$}

(1423 Oktober 13) Anno Domini $\mathbf{M}^{\circ} \mathbf{C C C C}^{\circ} \mathbf{X X I I I}^{\circ}$ in die Sancti Colomanni martyris in decanum facultatis assumptus est dominus Johannes Hess de Ysenach licenciatus in decretis, sub quo sunt intytulati infrascripti:

Item dom. Bohoslaus plebanus de Bano $2 \mathrm{gr}$.

Item mag. Johannes Preller plebanus in Trafess $3 \mathrm{gr}$.

Item Johannes Elrichshusen 2 gr.

70 Gesamter Eintrag von a. H.

71 In der Vorlage als Kopfzeile zu Beginn von fol. 19r eingetragen. 
Item Leonardus de Stayn 2 gr.

5 Item dom. Symon plebanus in Hanebach 2 gr.

Item Petrus Zirnperger 3 gr.

Item Bartholomeus Czedlicz de Hiersberg 3 gr.

Item frater Franciscus Althaymer lector ordinis Minorum in Nórdlinga 3 gr.

Promoti:

Item dom. Nicolaus Wenke prepositus Othmuchouiensis pro baccalariatu et licencia $4 \mathrm{fl}$.

10 Item mag. Johannes de Palborn pro baccalariatu $1 \mathrm{fl}$.

Item Heinricus Clodebogk pro baccalariatu $1 \mathrm{fl}$.

fol. $19^{v}$

$[1424 \mathrm{I}]$

Magister Caspar de Mayselstain doctor decretorum.

(1424 April 14) Anno Domini $\mathbf{M}^{\circ}$ quadringentesimo XXIIII' in die Sanctorum Tiburcii et Valeriani martyrum in decanum facultatis assumptus est dominus Caspar de Mayselstain decretorum doctor, sub quo sunt intitulati subscripti:

Item dom. Wenczeslaus de Wessele plebanus in Wlocznow 4 gr.

Item dom. Andreas Schónaw plebanus in Stubelaw 3 sol. d.

Johannes Hågg de Kirchain 3 gr.

Item Heinricus Megenhart de Blabưren 3 gr.

5 Item dom. Johannes Årkenstainer prepositus Gurnocensis 6 gr.

Item Mag. Michel de Schrick 3 gr.

Item Jacobus O̊ch de Pappenhaim 4 gr.

Item Vlricus Eysemman de O̊ttinga 4 gr.

Item Nicolaus Peck de Nyssa 3 gr.

10 Item Georius de Egerzeg Vngarus 2 gr.

Item Dominicus de Mo̊ner 4 gr.

Item dom. Johannes Hofrichter de Hallis 2 gr.

Item dom. Johannes Czwinger de Basilea 3 gr.

Item dom. Vlricus Stamhawser de Noua Ciuitate $3 \mathrm{gr}$.

15 Item mag. Luwertus Lankow de Danczk 4 gr.

Item dom. Nicolaus Hermanni de Fridlang 3 gr.

Item mag. Wenczeslaus de Montibus Kutnis 2 gr.

Item mag. Cristannus de Wasserburg 2 gr.

Item dom. Adolffus de Eppenstain Colonensis et Treuerensis canonicus, ${ }^{72}$

archidiaconus Treuerensis dt.

20 Item Vdalricus de Frankfordia pedagogus ipsius

Item dom. Fabianus de Tardeschedy pro licencia $3 \mathrm{fl}$.

Item mag. Petrus Pachmulner de Eberspewnt pro licencia $3 \mathrm{fl}$.

72 „1 fl.“ mittels Zuweisungslinie diesem und dem folgenden Eintrag zugeordnet. 
fol. $20^{r}$

Magister Petrus Pachmúlner licenciatus in decretis.

(1424 Oktober 13) Anno Domini millesimo etc. XXIIII' in die Sancti Colomanni martyris assumptus est in decanum facultatis magister Petrus Pachmulner de Eberspewnt licenciatus in decretis, sub quo infrascripti sunt intitulati:

Item mag. Michel de Göpping dt. 2 gr.

Item mag. Johannes Wilhelmi de Corona 2 gr.

Caspar Sigersdorffer 2 gr.

Item Petrus Ettenhayn de Argentina 2 gr.

5 Item dom. Alfonsus Gamezii canonicus Toletanus 3 sol. d.

Item mag. Seruacius de Mergenburg 2 gr.

Item dom. Johannes de Corona 2 gr.

Item dom. Nicolaus Heinrici de Praga archidiaconus Pragensis 8 gr.

Item dom. Michel de Rẽdisch altarista in Praga 2 gr.

Promoti sub eodem:

10 Item mag. Conradus Bladeck de baccalariatu $1 \mathrm{fl}$.

Item dom. Paulus Syndrami de Nissa de baccalariatu $1 \mathrm{fl}$.

Item dom. Thomas de Gelestorff de baccalariatu $1 \mathrm{fl}$.

Item dom. Fabianus de Tardaschedy de doctoratu $1 \mathrm{fl}$.

Item mag. Conradus de Langstat de baccalariatu $1 \mathrm{fl}$.

15 Item dom. Johannes Gerhardi de Ysenhusen de baccalariatu $1 \mathrm{fl}$.

fol. $20^{v}$

[1425 I]

Dominus Johannes Selde decretorum doctor.

(Nach 1425 April 14) Anno Domini Mo quadringentesimo XXV' post festum Sanctorum Tyburcii et Valeriani in decanum facultatis assumptus est dominus Johannes Selde decretorum doctor, sub quo sunt intytulati infrascripti:

Item Caspar Kalbskoph plebanus in Kunigsteten 2 gr.

Item dom. Georius Petri de Briswicz tavernicorum regalium magistri regni Vngarie ${ }^{73}$

Item Franciscus Johannis de Luko

Item Johannes Fuchs de Danczk 2 gr.

5 Item Bertoldus Tebershaymer canonicus ecclesie Sancti Johannis in Håwgis 2 gr.

Item Michael de Holabrunna 2 gr.

73 „1 fl. Vng.“ mittels Zuweisungslinie diesem und dem folgenden Eintrag zugeordnet. 


\section{Magister Paulus de Wienna.}

(Nach 1425 Oktober 13) Anno Domini Mo quadringentesimo $25^{\circ}$ post festum Sancti Colomanni martyris in decanum facultatis assumptus est magister Paulus de Wienna decretorum doctor, sub quo intitulati sunt:

Item Sigismundus de Styra monasterii de Gersten 2 gr. Item Johannes Sponsberg de Wratislauia 3 gr. Item mag. Erhardus Herrant de Newnburga de baccalariatu $1 \mathrm{fl}$. Item dom. Anthonius plebanus in Mulenbach de baccalariatu $1 \mathrm{fl}$.

5 Item dom. Andreas Michaelis de Wratislauia de baccalariatu $1 \mathrm{fl}$. Item dom. Petrus archidiaconus Transsiluanensis de licencia $3 \mathrm{fl}$. Item dom. Cholomanus Knapp canonicus ${ }^{74}$ Newnburgensis de baccalariatu $1 \mathrm{fl}$. Item dom. Martinus canonicus ${ }^{75}$ de Walthawsen pro baccalariatu $1 \mathrm{fl}$.

fol. $21^{r}$

[1426 I]

Dominus Johannes Gwërleich decretorum doctor.

(Nach 1426 April 14) Anno Domini millesimo quadringentesimo $26^{\circ}$ post festum Sanctorum Tyburcii et Valeriani in decanum facultatis est assumptus dominus Johannes Gwerleich decretorum doctor, sub quo intytulati sunt infrascripti:

Item dom. Petrus de Sulczpach canonicus ecclesie Wissegradensis 3 gr.

Item Gebso Heàwler de Constancia 4 gr.

Item mag. Heinricus Håppel 3 gr.

Item Johannes Hoffkircher de Wienna 3 gr.

5 Item Conradus Herbeyn de Tywing 2 gr.

Item Cristannus Mulpacher de Kufstain 2 gr.

Item dom. Michael Stankonis de Wratislauia 2 gr.

Item dom. Ladislaus archidiaconus ecclesie Transsiluanie 4 sol. d.

Promoti:

Item mag. Thomas O̊der de Achspach de licencia $3 \mathrm{fl}$.

10 Item mag. Conradus Bladeck de Wienna $3 \mathrm{fl}$.

Item mag. Johannes Ambrosii pro baccalariatu $1 \mathrm{fl}$.

Item dom. Johannes canonicus regularis Ordinis Premonstratensis monasterii Roggenburgensis de baccalariatu $1 \mathrm{fl}$.

74 Von a. H. über der Zeile eingefügt.

75 Von a. H. über der Zeile eingefügt. 
fol. $21^{v}$

[1426 II]

(1426 Oktober 13) Anno Domini millesimo quadringentesimo vigesimo sexto in die Sancti Colomanni martyris in decanum facultatis assumptus est magister Thomas Ôder de Aspach licenciatus in decretis, sub quo infrascripti sunt intitulati:

Mag. Jeroslaus de Sluskow canonicus Cracouiensis dt. 3 sol. d.

Mag. Petrus Mürbacher de Lincz dt. 3 gr.

Mag. Petrus de Lantshuta dt. 2 gr.

Mag. Dyonisius de Newburga dt. 2 gr.

5 Nicolaus Paskowicz plebanus in Kant dt. 2 gr.

Petrus Newsidler de Laa dt. 2 gr.

Seruacius de Corona dt. 2 gr.

Bartolomeus de Martpurga p.

Jacobus de Chweffstain dt. 2 gr.

10 Conradus Reintaler de Oberberga dt. 2 gr.

Caspar de Brezewicz Sepusiensis dt. 2 gr.

Mag. Lucas Slitt de Prün dt. 2 gr.

Albertus de Keczkemeth dt. 2 gr.

Johannes de Zagrabia bacc. in artibus dt. 3 gr.

15 Petrus de Hamelburg bacc. in artibus dt. 2 gr.

$\left.\begin{array}{l}\text { Dom. }{ }^{76} \text { Johannes de Ebersdorff dt. }{ }^{77} \\ \text { Sigismundus Walich de Aybling dt. }\end{array}\right\} 1 \mathrm{fl}$.

Matheus de Kulled dt. 3 gr. ${ }^{78}$

fol. $22^{r}$

Promoti in eodem decanatu:

Mag. Cristoforus Guldein de Rain pro baccalariatu $1 \mathrm{fl}$.

20 Dom. Conradus de Elbang pro baccalariatu $1 \mathrm{fl}$.

Dom. Bohoslaus plebanus de Bano pro baccalariatu $1 \mathrm{fl}$.

Jacobus de Chuefstain pro baccalariatu $1 \mathrm{fl}$.

[1427 I]

(Nach 1427 April 14) Anno Domini M CCCC vigesimo septimo post festum Sanctorum Tiburcii et Valerii martirum in decanum facultatis iuris canonici assumptus est magister Conradus Bladek de Wienna licenciatus in decretis, sub quo intitulati sunt infrascripti:

76 Von s. H. am linken Außenrand.

77 „1 fl.“ mittels Zuweisungsstrich diesem und dem folgenden Eintrag zugeordnet.

78 Gesamter Eintrag von a. H. 
Dom. Johannes de Pudlino canonicus ecclesie collegiate Sancti Martini ${ }^{79}$

Gabriel de Augusta gr. 2

Mag. Johannes de Bruchenstain gr. 8

Bernhardus frater Ordinis Sancti Johannis gr. 2

5 Leutherus de Slesia ${ }^{80}$

Heinricus Prepticz de Slesia $\}$ obligatur

Heinricus Roraw gr. 6

Mag. Thomas de Vttendorff gr. 2

Stephanus de Walthausen gr. 2

10 cum domino Leonhardo prope scolas iuris[tarum] gr. 2

Promoti in eodem decanatu:

Dom. Johannes de Brixna pro baccalariatu $1 \mathrm{fl}$.

fol. $22^{v}$

[1427 II]

(Nach 1427 Oktober 13) Anno Domini M CCCC XXVII post festum Sancti Kolomanni martiris in decanum facultatis iuris canonici assumptus est magister Conradus de Hallstat decretorum [doctor], sub quo intitulati sunt infrascripti:

Johannes de Gemundia gr. 2

Dom. Johannes archidyaconus Newgradiensis in ecclesia Strigoniensi ac canonicus ecclesie Transiluanensis fl. 1/2

Henricus Klodechk gr. 2

Dom. Johannes [de] Grecz Regine decanus ecclesie collegiate Kremsirensis ac canonicus ecclesie Bratislauiensis fl. 1/2

5 Dom. Ladislaus de Schlak bacc. in artibus, canonicus ecclesie Baradiensis fl. 1

Dom. Antonius lic. in artibus de Muschna gr. 2

Martinus Phaffenhofer de Sancto Floriano procurator scole iuristarum

$\left.\begin{array}{l}\text { Conradus de Vlma } \\ \text { Johannes de Vlma }\end{array}\right\}$ bacc. in artibus $\left\{\begin{array}{l}2 \mathrm{gr}^{82} \\ 2 \mathrm{gr}^{83}\end{array}\right.$

Promoti in eodem decanatu:

10 Mag. Vdalricus de Weyssenburga pro licencia fl. 3

Dom. Andreas canonicus ecclesie Agriensis pro licencia fl. 3

Dom. Antonius plebanus in Mùlbach pro licencia fl. 3

Dom. Johannes Råbel de Corona pro baccalariatu fl. 1

79 Angabe der Taxen fehlt

80 „obligatur“ mittels Zuweisungslinien diesem und dem folgenden Eintrag zugeordnet.

81 „bacc. in artibus“ mittels Zuweisungslinien diesem und dem folgenden Eintrag zugeordnet.

82 Gesamter Eintrag mit hellerer Tinte nachgetragen.

83 Gesamter Eintrag mit hellerer Tinte nachgetragen. 
Dom. Georius ${ }^{84}$ de Vngaria pro baccalariatu fl. 1

15 Dom. Caspar de Wolffsperg pro baccalariatu fl. 1

fol. $23^{r}$

[1428 I]

(Nach 1428 April 14) Anno ${ }^{85}$ Domini millesimo quadringentesimo vigesimo octavo post festum Sanctorum martyrum Tiburcii et Valeriani in decanum facultatis iuris canonici assumptus est magister Vlricus de Weyssenburga licenciatus in decretis, sub quo intitulati sunt infrascripti:

Johannes Mưnheym ${ }^{86}$ de Praga dt. 4 gr.

Petrus Fabri de Ostrauia dt. 2 gr.

Albertus de Waradino lic. in artibus dt. 2 gr.

Johannes Polzmacher de Brünna dt. 2 gr.

5 Johannes Rötlini de Opauia dt. 2 gr.

Mag. Jeronimus Lantuoyt de Olmüncz dt. 2 gr.

Dom. Nicolaus Scho̊nperger de Olmüncz dt. 2 gr.

Dom. Jacobus de Clausenburk dt. 3 gr.

Johannes Screyber de Weyssenburga

Promoti in eodem decanatu:

10 Dom. Petrus Nobag dt. pro licencia $3 \mathrm{fl}$.

Dom. Anthonius plebanus in Můlnpach pro doctoratu $1 \mathrm{fl}$.

Item dom. Petrus Nobag pro doctoratu fl.

Dom. Dominicus de Mo̊lnar pro baccalariatu $1 \mathrm{fl}$.

Dom. Ladislaus archidiaconus ecclesie Transsiluanensis pro baccalariatu $1 \mathrm{fl}$.

15 Dom. Petrus de Sulczpach canonicus ecclesie Sagrabiensis $1 \mathrm{fl}$.

fol. $23^{v}$

$[1428 \mathrm{II}]$

(Nach 1428 Oktober 15) Anno Domini millesimo quadringentesimo vicesimo octavo feria sexta post festum Sancti Colomanni martiris in decanum facultatis iuris canonici assumptus est magister Paulus de Wienna decretorum doctor, sub quo intitulati sunt infrascripti:

Johannes Resch de Swabach bacc. in artibus 2 gr.

Andreas Langkhaim de Prussia 2 gr.

Matheus Slick prepositus Boleslauiensis ecclesie collegiate 1/2 lb.

84 Davor "Jo" getilgt.

85 Am rechten oberen Rand dieser Seite „1428“, wohl aus derselben Zeit stammend.

86 Davor „de“" getilgt. 
Stephanus de Abara canonicus Agriensis 3 sol. d.

5 Mag. Andreas Santperg 2 gr.

Mag. Wilhelmus Grayspach de Monaco 2 gr.

Georius Jågenrểwtter de Patauia 2 gr.

$\left.\begin{array}{l}\text { Dom. Martinus archidiaconus de Hunyad ecclesie Transsiluanensis }{ }^{87} \\ \text { David de Gyrold famulus domini archidiaconi }\end{array}\right\} 3$ sol. d.

10 Frater Caspar Ebenhawser canonicus ecclesie Pawburgensis ${ }^{88} 4 \mathrm{gr}$.

Promoti in eodem decanatu:

Dom. Johannes de Bozna canonicus Casmensis pro baccalariatu $1 \mathrm{fl}$.

Dom. Colmannus Knapp canonicus Newmburgensis pro licencia $3 \mathrm{fl}$.

Dom. Martinus canonicus in Walthawsen pro licencia $3 \mathrm{fl}$.

Dom. Alfonsus Gomecii canonicus ecclesie Toletanensis pro baccalariatu $1 \mathrm{fl}$.

fol. $24^{r}$

$[1429 \mathrm{I}]$

(Ca. 1429 April 14) Anno Domini millesimo quadringentesimo vicesimo nono circa festum Sanctorum Tyburcii et Valeriani in decanum facultatis iuris canonici assumptus est dominus Johannes Seld decretorum doctor, sub quo intitulati sunt infrascripti:

Johannes Hers canonicus ecclesiarum Brunnensis et Olomuncensis, bacc. in artibus 5 gr.

Mag. Franciscus Woiczdorff bacc. in theologia, canonicus ecclesie collegiate Libnicensis 4 gr.

Dom. Johannes Toliatoris de Crossna 2 gr.

Nicolaus Jenkebicz de Woslawicz 2 gr.

5 Dom. Johannes plebanus in Nouoforo prope Frisacum 3 gr.

Dom. Demetrius Czuporis archidiaconus de Kykuw, canonicus in ecclesia Transsiluana ${ }^{89}$

Mag. Ciriacus de Quinque [Ecclesiis] etc.

Dom. Stephanus Mardax 4 gr.

Allexius Strenberger 2 gr.

10 Mag. Laurencius de Corona 4 gr.

Albertus Slewser bacc. in artibus 3 gr.

Mag. Johannes Czap de Bechthem 2 gr.

Mag. Vlricus Czap de Bechthem 2 gr.

Dom. Petrus Cziller 2 gr.

15 Johannes Poter Sartoris de Fridwerkch

Johannes Jeger 2 gr.

87 „3 sol. d.“ mittels Zuweisungslinien diesem und dem folgenden Eintrag zugeordnet.

88 Am linken Seitenrand von a. H. „prepositus ibidem nunc“.

89 „1 f." mittels Zuweisungslinien diesem und dem folgenden Eintrag zugeordnet. 
Vlricus Ruch 2 gr.

fol. $24^{v}$

$[1429$ II]

(Nach 1429 Oktober 13) Anno Domini millesimo quadringentesimo vicesimo nono post festum Sancti Colomanni martiris in decanum facultatis iuris canonici assumptus est magister Conradus de Halstat decretorum doctor, sub quo intitulati sunt infrascripti:

Dom. Vlricus comes de O̊tingen cum domino Fridrico et Georio ${ }^{90}$ etc. 1 fl. Vng.

Mag. Jacobus Tols de Rudlinga 2 gr.

Mag. Martinus de Leibitz 2 gr.

Conradus Kuenlein de Dyeppurg

5 Johannes Aichelperger de Nouoforo 2 gr.

Dom. Fridricus Sartoris alias Maler rector ecclesie parrochialis in Pirchachhausen

1 fl. Vng. [Vgl. oben Eintrag 1.]

Georius Plóntinger [vgl. oben Eintrag 1.]

Mag. Jeronimus de Olmuncz 2 gr.

Deobaldus de Frisinga 2 gr.

10 Hilprandus Oder de Dorffen 2 gr.

Johannes Arnolt de Tullaco 4 gr.

Cristofferus Arnolt de Tullaco 4 gr.

Frater Vdalricus de Augusta Ordinis Sancti Benedicti 4 gr.

Dom. Tieboldus Aichperger canonicus ecclesie Frisingensis 3 sol.

15 Mag. Conradus Pachenstain 5 gr.

Gregorius Molitoris de Noua Ciuitate 2 gr.

Theodricus de Fridland 2 gr.

Mag. Nicolaus Reyman de Olsna *9 gr.*

Johannes Nicolai Fabri de Olsna ${ }^{*} 9$ gr.*

20 Cristannus de Hiltenstorff de Wurcia 2 gr.

Dom. Petrus Kieler plebanus in Campidona 4 gr.

Frater Leonhardus Tenniger de Monaco Ordinis Minorum $20 \mathrm{~d}$.

Dom. Johannes Hieler de Ala licenciandus in artibus 2 gr.

Johannes Gotz de Studkardia 2 gr.

25 Luthardus de Chonstatt 2 gr.

Wilhelmus Steger de Mittenbald 1 gr.

Promotus in eodem decanatu dom. Hainricus plebanus in Scheibs pro licencia dt. $3 \mathrm{fl}$.

90 Vgl. die Einträge 6 und 7 in diesem Semester, auf welche in der Vorlage mittels einer Linie am linken Seitenrand verwiesen wird. 
fol. $25^{r}$

$[1430 \mathrm{I}]$

(Nach 1430 April 14) Anno Domini millesimo quadringentesimo tricesimo post festum Sanctorum Tiburcii et Valeriani in decanum facultatis iuris canonici assumptus est dominus Johannes Gwerleich decretorum doctor, sub quo intitulati sunt infrascripti:

Johannes Koslick de Podatzschin 2 gr.

Conradus Seydenliep de Erfordia 2 gr.

Stephanus de Wusla 2 gr.

Dom. Johannes Schiltdorffer plebanus in Gastpoltshouen 4 gr.

5 Johannes Rademan de Lukau altarista ecclesie Wratislauiensis 3 gr.

Pangracius Conradi de Sweinbart 2 gr.

Mag. Anthonius de Sintauia 2 gr.

Frater Wernherus professus monasterii in Elbang 2 gr.

Dom. Jacobus de Rosenaw 3 gr.

10 Dom. Petrus Hebringer plebanus ad Sanctum Andream in Goss 4 gr.

Dom. Nicolaus Stubner canonicus Agriensis 3 sol.

Mag. Florianus de Leibnitz 3 gr.

Dom. Thomas Hym de Brente canonicus ecclesie Vespermiensis 6 gr.

Mag. Hainricus Plesner de Eschenbach 2 gr.

15 Johannes Pusmer de Nurmberga 2 gr.

Dom. Rabanus de Lybenstain ${ }^{91}$ )

$\left.\begin{array}{l}\text { Dom. Richardus de Superiori } \\ \text { Lapide }\end{array}\right\}$ canonici ecclesie Maguntinensis dederunt $1 \mathrm{fl}$.

Conradus de Pemeshaim 2 gr.

Petrus de Pemeshaim 2 gr.

20 Dom. Conradus Arnoldi canonicus ecclesie Sancti Stephani Maguntinensis 2 gr.

Dom. Mathias Schrat plebanus in Pechstal 3 gr.

Dom. Heinricus de Campa decanus ecclesie Opoliensis 6 gr.

Promoti in eodem decanatu:

Dom. Alfansus pro licencia $3 \mathrm{fl}$.

Idem Alfansus pro doctoratu $1 \mathrm{fl}$.

25 Mag. Petrus Pachmulner pro doctoratu $1 \mathrm{fl}$.

Dom. Wolfgangus Payr plebanus in Czisterstorf pro baccalariatu $1 \mathrm{fl}$.

Dom. Hainricus Klodebokch pro licencia $3 \mathrm{fl}$.

Mag. Johannes Pachenstain pro baccalariatu $1 \mathrm{fl}$.

Mag. Franciscus pro baccalariatu $1 \mathrm{fl}$.

30 Mag. Erhardus Herrant ${ }^{92}$ pro licencia $3 \mathrm{fl}$.

Dom. Georgius pro licencia $3 \mathrm{fl}$.

91 Vorlage: von „canonici“ bis „1 fl.“ mittels Zuweisungslinien diesem und dem folgenden Eintrag zugeordnet.

92 Von a. H. über der Zeile nachgetragen. 
Dom. ${ }^{93}$ Martinus archidiaconus Transsiluaniensis pro baccalariatu $1 \mathrm{fl}$.

Mag. Jeronimus de Olmuncz pro baccalariatu $1 \mathrm{fl}$.

Dom. Johannes Poltzmacher de Brunna pro baccalariatu $1 \mathrm{fl}$.

fol. $25^{v}$

$[1430 \mathrm{II}]$

(Nach 1430 Oktober 13) Anno Domini $\mathbf{M}^{\circ}$ CCCC $^{\circ}$ XXX $^{\circ}$ post festum Sancti Colomanni martiris in decanum facultatis iuris canonici assumptus est magister Erhardus Herrant licenciatus in decretis, sub quo sunt intitulati infrascripti:

Hermannus Huxer de Pruscia dt. 2 gr.

Paulus Mankelwicz ${ }^{94}$ de Legnicz dt. 2 gr.

Mag. Vlricus Sunperger de Orengew dt. 2 gr.

Johannes Maulperger de Altenmuldarff p.

5 Dom. Michael de Boden canonicus ecclesie Transsiluanensis 3 sol. d.

Dom. Simon de Bacia Agriensis et Quinqueecclesiensis ecclesiarum canonicus 3 sol.

d.

Dom. Sigismundus Cisterdarffer dt. 4 gr.

Dom. Johannes Stawdhaymer de O̊tting 2 gr.

Stephanus Neumann de Freinstat 2 gr.

10 Dom. Vicencius plebanus in Haydendarff 4 gr.

Mag. Michael de Priuidia 2 gr.

Mag. Johannes de Sindelfing 2 gr.

Mag. Emericus custos et canonicus ecclesie Baciensis 3 sol. d.

Nicolaus Kynsperger de Chrewsen 2 gr.

15 Dom. Cristannus plebanus in Villa Hainperti 2 gr.

Dom. Anthonius plebanus in Hamlach 4 gr.

Mag. Johannes Rătelchouer 2 gr.

Martinus Pratesser 2 gr.

Georius plebanus in Schatschelkch 2 gr.

20 Andreas plebanus in Redsch 4 gr.

Conradus Emmen de Hildeshaym 2 gr.

Dom. Conradus Reykershouer Ratispanensis et Eystetensis ecclesiarum canonicus dt. 3 sol. d.

Erhardus Fabri canonicus ecclesie Sancti Viti Heridensis 2 gr.

Wolfgannus Temler de Stira p.

fol. $26^{r}$

Promoti in eodem decanatu:

93 Die letzten drei Eintragungen dieses Semesters wurden wegen Platzmangels mittels Verweiszeichen an das Ende der vorhergehenden Seite (fol. $24^{\mathrm{v}}$ ) gesetzt.

94 „“"von s. H. eingefügt. 
25 Dom. Caspar canonicus ecclesie Strigoniensis pro baccalariatu $1 \mathrm{fl}$.

Dom. Johannes de Chrussen pro baccalariatu $1 \mathrm{fl}$.

Dom. Heinricus Feuchter pro baccalariatu $1 \mathrm{fl}$.

Dom. Petrus presbyter pro baccalariatu $1 \mathrm{fl}$.

Dom. Jacobus de Chlausenburga pro baccalariatu $1 \mathrm{fl}$.

30 Mag. Albertus de Baradino pro baccalariatu $2 \mathrm{fl}$.

Dom. Mathias Slikch prepositus Bolislauiensis pro baccalariatu $2 \mathrm{fl}$.

Mag. Laurencius de Septemcastris pro baccalariatu $1 \mathrm{fl}$.

Dom. Colomannus canonicus ecclesie Neuburgensis pro doctoratu $1 \mathrm{fl}$.

Dom. Martinus canonicus in Walthusen pro doctoratu $1 \mathrm{fl}$.

35 Dom. Petrus Agmanni pro doctoratu $1 \mathrm{fl}$.

Dom. Georius Egersek pro doctoratu $1 \mathrm{fl}$.

$[1431 \mathrm{I}]$

(1431 Mai 10) In die Ascensionis Domini.

(Nach 1431 April 14) Anno Domini millesimo XXXI post festum Sancti Tiburcii et Valeriani martyrum assumptus est in decanum facultatis magister Petrus Pachmúlner decretorum doctor, sub quo sunt intitulati infrascripta supposita:

Mag. Hertwicus Lampoltinger dt. 4 gr.

Petrus Chramer plebanus in Anaso dt. 40 d.

Vlricus Lachmair dt. 3 gr.

Petrus O̊denfurter de Liechtenburg dt. 2 gr.

5 Mag. Andreas de Laybaco dt. 2 gr.

fol. $2 \sigma^{v}$

Caspar Czener de Patauia dt. 16 d. ${ }^{95}$

Mag. Laurencius de Obernburg dt. 2 gr.

Paulus Glen de Noua Ciuitate p.

Johannes Stainger de Monaco p.

10 Dom. Barnabas de Mera dt. 3 sol. d.

Promoti sub eodem: ${ }^{96}$

Mag. Thomas de Vttendorf dt. $1 \mathrm{fl}$.

Jeorius Jăgenreiter dt. $1 \mathrm{fl}$.

Dom. Ladislaus de Czschach dt. 2 fl.

Martinus Czeller dt. 1 fl.

15 Conradus de Vlma dt. 1 fl.

95 Es folgt ein getilgter und weiter unten wiederholter Eintrag: „Conradus de Vlma dt. $1 \mathrm{fl}$. pro baccalariatu“.

96 Von s. H. am linken Seitenrand eingefügt. 
(Nach 1431 Oktober 13) Anno Domini $\mathbf{M}^{\circ} \mathbf{C C C C}^{\circ} \mathbf{X X X I}^{\circ}$ post festum Sancti Colomanni martyris assumptus est in decanum facultatis magister Paulus de Wienna decretorum doctor, sub quo intitulati et promoti sunt infrascripti:

Item dom. Vdalricus de Haydreichstain magister chori ad Sanctum Stephanum Wiennensem 4 gr.

$\left.\begin{array}{l}\text { Item dom. Petrus de Palocz mag. in artibus }{ }^{97} \\ \text { Item Antonius de Busa canonicus ecclesie Sancti Thome in Strigonio }\end{array}\right\} 1 \mathrm{fl}$.

Item dom. Stephanus Feyr de Clawsemburg 3 gr.

5 Item Nicolaus Erlacher de Purkhawsen 2 gr.

Item Laurencius Lewbner filius Nicolai de Nissa 2 gr.

Item dom. Georius de Wacia canonicus ecclesie Waciensis 3 sol. d.

Item dom. Georius Stẻninger canonicus ecclesie Beate Marie in Fewchtwang 4 gr.

Item dom. Nicolaus plebanus ad Sanctum Vdalricum in Noua Ciuitate extra muros $4 \mathrm{gr}$.

fol. $27^{r}$

10 Item dom. Petrus Fuchs professus monasterii in Schussenried Ordinis Premonstratensis 6 gr.

Item dom. Thomas de Norap canonicus ecclesie Jauriensis 3 sol.

Item Mag. Johannes de Wincz plebanus in Foro Ruthenorum 6 gr.

Item dom. Clemens plebanus ecclesie in Dobrica 6 gr.

Item Johannes de Redisch bacc. in artibus 2 gr.

15 Item dom. Conradus de Kreyg prepositus ecclesie in Volkenmarkt

Item dom. Heinricus Hülger de Eberspewnt 2 gr.

Nota promotos:

Item dom. Ladislaus de Strig archidiaconus ecclesie Transsiluanensis de licencia $3 \mathrm{fl}$. Item Mag. Johannes de Bachenstain archidiaconus ecclesie Zagrabiensis de licencia 3 fl.

Item dom. Johannes de Bossna canonicus ecclesie Zagrabiensis de licencia $3 \mathrm{fl}$.

20 Item Mag. Jodocus Rot de Vberling pro baccalariatu $1 \mathrm{fl}$.

Item dom. Nicolaus de Scho̊nberg pro baccalariatu $1 \mathrm{fl}$.

Item dom. Johannes Czopot plebanus in Nouoforo pro baccalariatu $1 \mathrm{fl}$.

Item dom. Caspar Ebenhawser canonicus regularis in Pawmburg pro baccalariatu $1 \mathrm{fl}$.

Item dom. Conradus Künlein de Dyppurg $1 \mathrm{fl}$.

25 Item dom. Johannes Czepeck plebanus ad Sanctum Vitum prope Laybacum pro baccalariatu $1 \mathrm{fl}$.

97 „1 fl.“ mittels Zuweisungslinien diesem und dem folgenden Eintrag zugeordnet. 
(1432 April 27) Anno Domini millesimo quadringentesimo XXXII ${ }^{\circ}$ die vigesima septima mensis Aprilis electus est in decanum facultatis iuris canonici magister Johannes Gwerleich decretorum doctor, sub quo intitulati et promoti sunt infrascripti:

Item dom. Conradus de Chreig prepositus in Volkenmarckt 3 sol.

Item dom. Caspar canonicus regularis ad Sanctum Ypolitum 3 sol.

Item dom. Michael Steger 3 gr.

Item Vlricus Hoffner bacc. arcium 2 gr.

5 Item dom. Andreas canonicus monasterii Neunburgensis 5 gr.

Item dom. Bernardus cappelanus in curia Newnburgensi $4 \mathrm{gr}$.

fol. $27^{v}$

Item venerabilis et egregius vir dom. Hartungus de Cappell utriusque iuris dr. ordinarius etc. pro incorporacione facultatis $1 \mathrm{fl}$.

Item Mag. Andreas Feynockh de Berchtoltstorf 2 gr.

Item dom. Nicolaus Rebiczer canonicus Ratisponensis 8 gr.

10 Item Johannes de Altamuta 2 gr.

Item Mag. Henricus Slick prepositus in Northusen 3 sol.

Item Mag. Petrus de Rauenspurg 3 gr.

Item Johannes Raptoris 2 gr.

Item dom. Thomas de Creyuelt plebanus in Pilichtorf 6 gr.

Secuntur promoti:

15 Item Mag. Erhardus Herrannt pro doctoratu $1 \mathrm{fl}$.

Item Mag. Johannes de Bachenstain canonicus Zagrabiensis pro doctoratu $1 \mathrm{fl}$.

Item dom. Johannes de Zagrabia pro doctoratu $1 \mathrm{fl}$.

Item dom. Johannes Aichelperger de Nouoforo pro baccalariatu $1 \mathrm{fl}$.

Item dom. Johannes Hofleich pro baccalariatu $1 \mathrm{fl}$.

20 Item dom. Johannes Has canonicus Olomuncensis pro baccalariatu $1 \mathrm{fl}$.

\section{[1432 II]}

(Nach 1432 Oktober 13) Anno Domini $\mathbf{M}^{\circ}$ quadringentesimo XXXII ${ }^{\circ}$ post festum Sancti Colomanni martiris assumptus est in decanum facultatis iuris canonici dominus Petrus Nowag decretorum doctor et canonicus ecclesie Transiluane, sub quo intitulati et promoti sunt infrascripti:

$\left.\begin{array}{l}\text { Dom. Albertus comes de O̊tingen } \\ \text { Item Mag. Johannes Graw }\end{array}\right\} 1$ fl.

Item Mag. Johannes Czeller 2 gr.

Item Cristannus de Molinpach p. ${ }^{99}$

98 „1 fl.“ mittels Zuweisungslinien diesem und dem folgenden Eintrag zugeordnet.

99 „p." von a. H. auf Rasur. Von dieser Hand auch der Nachtrag „postea in anno 49 dt. 2 gr.“ 
5 Item Johannes Telkener de Cremniczia bacc. in artibus 2 gr.

Item Johannes Wingler de Medling bacc. in artibus 2 gr.

Item Johannes Stange de Gorlicz 2 gr.

fol. $28^{r}$

Sequuntur promoti in eodem decanatu:

Primo dom. Johannes Polczmacher pro licencia $3 \mathrm{fl}$.

Item dom. Antonius plebanus in Omlaschz de Septemcastris pro baccalariatu $1 \mathrm{fl}$.

10 Item Mag. Jacobus de Strigonio pro baccalariatu $1 \mathrm{fl}$.

Item Mag. Albertus de Waradino archidiaconus et canonicus ecclesie Strigoniensis pro licencia $3 \mathrm{fl}$.

Item dom. Petrus de Laa pro licencia 3 fl. ${ }^{100}$

(Nach 1433 April 14) Anno Domini Mo CCCC XXXIII post festum Sanctorum Tiburcii et Valeriani assumptus est in decanum facultatis iuris canonici dominus Hartungus de Cappel utriusque iuris doctor, canonicus ecclesie Patauiensis, sub quo intitulati et promoti sunt infrascripti:

Dom. Nicolaus Czink quondam prepositus in Varaw sol. 3

Item dom. Johannes Briger canonicus ecclesie Wratislauiensis sol. 3

Item dom. Thomas Angelpekh procurator ecclesie ad Sanctum Leonardum in Foresta gr. 12

Item dom. Johannes Quentin de Ortenberg gr. 2

5 Item mag. Vdalricus Lang de Augusta gr. 2

Item mag. Johannes de Hallstat gr. 2

Item dom. Philippus Swarat de Brixina gr. 3

Item dom. Johannes Stadelmair p.

Item mag. Nicolaus de Zagrabia gr. 2

10 Item dom. Valentinus de Mendscenth gr. 2

Item dom. Hainricus Wismayr gr. 2

Sequuntur promoti in eodem decanatu:

Primo dom. Nicolaus Stubner canonicus Agriensis pro baccalariatu fl. 1

Item mag. Anthonius fl. 1

Item frater Vdalricus de Augusta Ordinis Sancti Benedicti pro baccalariatu fl. 1

15 Item mag. Gregorius Scharendorf de Stuckardia fl. 1

100 Danach ein Eintrag durch Rasur getilgt und unleserlich. 


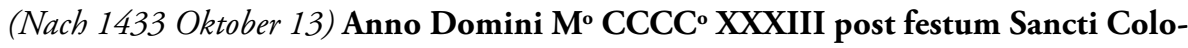
manni martiris assumptus est in decanum facultatis iuris canonici magister Erhardus Herrant decretorum doctor, officialis curie Patauiensis, sub quo sunt intitulati et promoti infrascripti:

Nicolaus Reychel de Wratislauia 2 gr.

Philippus Gladiatoris 2 gr.

Mag. Arbogastus 2 gr.

Johannes Parrewtter 2 gr.

5 Mag. Nicolaus Bethneri canonicus regularis monasterii Beate Virginis

Wratislauie in Arena

et Sigismundus Aczo familiaris eius

Secuntur promoti:

Dom. Andreas plebanus in Recz de Septemcastris pro baccalariatu $1 \mathrm{fl}$.

Dom. Cristannus plebanus in Villa Hainperti de Septemcastris pro baccalariatu $1 \mathrm{fl}$.

Frater Berngerus professus monasterii in Elbangen pro baccalariatu $1 \mathrm{fl}$.

10 Dom. Johannes Resch dt. ad baccalariatum $1 \mathrm{fl}$.

Frater Petrus Ordinis Premonstratensis monasterii ${ }^{101}$ pro baccalariatu $1 \mathrm{fl}$.

Dom. Georius plebanus de Schadschek Septemcastrensis pro baccalariatu $1 \mathrm{fl}$.

Mag. Laurencius de Obernburk pro baccalariatu $1 \mathrm{fl}$.

Mag. Jeronimus Landfoyd de Olmuncz pro licencia $3 \mathrm{fl}$.

15 Dom. Georius Jegenrewtter plebanus in Varichdarff pro licencia $3 \mathrm{fl}$.

Mag. Caspar de Piburga 2 gr.

Dom. Petrus de Weydenpach 2 gr.

Dom. Georius de Corona 2 gr.

fol. 29 r

$[1434$ I]

(1434 April 25) Anno Domini $\mathbf{M}^{\circ} \mathbf{C C C C}^{\circ} \mathbf{X X X I I I I}^{\circ}$ die $\mathbf{X X V}^{\mathbf{a}}$ mensis Aprilis assumptus est in decanum facultatis iuris canonici magister Conradus de Halstat decretorum doctor, sub quo intitulati et promoti sunt infrascripti:

Mag. Johannes de Tapolcza canonicus ecclesie Waradiensis 3 sol. d.

Dom. Jacobus Rayner plebanus in Cirkla 2 gr.

Johannes de Chochaim 2 gr.

Dom. Petrus plebanus de Můschna de Septemcastris 3 sol. d. 
5 Cristannus Ewart de Stolczemberg 2 gr.

Dom. Johannes Mulhaimer plebanus in Aussernczelln 8 gr.

Mag. Nicolaus Stürpecher de Glocz 2 gr.

Dom. Marcus Lessna de Maiori Glogouia 2 gr.

Dom. Johannes Stremphel de Weissemburga bacc. in artibus 3 gr.

10 Dom. Michael Ekhart de Ardisch de Septemcastris 4 gr.

Dom. Caspar plebanus in Veklaprugk 8 gr.

Dom. Petrus plebanus Sancti Georii super Ybsueld 4 gr.

Wolfgangus Sachs de Almekch prope Lambach 4 gr.

Johannes Pluemenekcher de Argentina bacc. in artibus 2 gr.

Promoti:

15 Mag. Petrus de Rauenspurg pro baccalariatu $1 \mathrm{fl}$.

Mag. Arbogastus Elhart pro baccalariatu $1 \mathrm{fl}$.

Mag. Michael de Priuidia pro baccalariatu $1 \mathrm{fl}$.

Dom. Johannes Rademan pro baccalariatu $1 \mathrm{fl}$.

Dom. Conradus Reickershofer canonicus ecclesie Ratisponensis pro baccalariatu $1 \mathrm{fl}$.

fol. $29^{v}$

$[1434 \mathrm{II}]$

(Nach 1434 Oktober 13) Anno Domini M $^{\circ}$ CCCC $^{\circ}$ XXXIIII' ${ }^{\text {to }}$ post festum Beati Colomanni martiris assumptus est in decanum facultatis iuris canonici magister Petrus Pachmúlnể decretorum doctor, sub quo sunt intitulati et promoti infrascripti:

Dom. Ladislaus de Hederfar cantor et canonicus ecclesie Vesprimiensis dt. 1 lb. d.

Mag. Nicolaus Lepes archidiaconus et canonicus ecclesie Transsiluanensis dt. $1 \mathrm{lb}$. d.

Cristoforus Dachsawer dt. 4 gr.

Johannes Reysegk de Reysspach dt. 3 gr.

5 Andreas de Myschkolcz dt. 3 gr.

Mag. Thomas de Tulna dt. 2 gr.

Gregorius Weytensdorffer dt. 2 gr.

Secuntur promoti:

Mag. Jacobus de Strigonio pro licencia dt. $3 \mathrm{fl}$.

Johannes Sews pro baccalariatu dt. $1 \mathrm{fl}$.

10 Mag. Vlricus de O̊ringew pro baccalariatu dt. $1 \mathrm{fl}$.

Caspar Czener pro baccalariatu dt. $1 \mathrm{fl}$.

Mag. Jacobus de Strigonio pro doctoratu dt. $1 \mathrm{fl}$.

Dom. Johannes Polczmacher pro doctoratu dt. $1 \mathrm{fl}$.

Mag. Franciscus de Legnitz pro licencia dt. $3 \mathrm{fl}$.

15 Idem [Franciscus de Legnitz] pro doctoratu dt. $1 \mathrm{fl}$.

Mag. Albertus de Waradino pro doctoratu dt. $1 \mathrm{fl}$. 
(1435 April 22) Anno Domini Mo CCCC XXXV die Veneris XXII. mensis Aprilis assumptus est in decanum facultatis iuris canonici venerabilis vir dominus Johannes de Eych utriusque iuris doctor, sub quo sunt intitulati et promoti infrascripti:

Intitulati:

Mag. Georgius Hohenloch de Bamberga 2 gr.

Mag. Gregorius de Valle Sancti Georgii canonicus ecclesie Strigoniensis 3 sol.

Dom. Johannes Rủel de Francfordia 5 gr.

Leonhardus Kellner de Wirgil 2 gr.

5 Vlricus Waltheri de Streifdorf 2 gr.

Dom. Gabriel Lang de Augusta 3 gr. ${ }^{102}$

Secuntur promoti:

Dom. Conradus Sybmer pro baccalariatu $1 \mathrm{fl}$.

Dom. Nicolaus Erlacher de Purkhusa pro baccalariatu $1 \mathrm{fl}$.

Dom. Caspar canonicus regularis de Sancto Yppolito pro baccalariatu $1 \mathrm{fl}$.

10 Dom. Johannes Aychelperger pro licencia $3 \mathrm{fl}$.

Dom. Conradus Reykershofer canonicus Ratisponensis pro licencia $3 \mathrm{fl}$.

Dom. Michael Hylsmar de Stolczemberg pro licencia $3 \mathrm{fl}$.

Dom. Nicolaus Stůbner canonicus Agriensis pro licencia $3 \mathrm{fl}$.

Mag. Anthonius de Schnitauia pro licencia $3 \mathrm{fl}$.

15 Dom. Fredericus Sartoris alias Maler plebanus in Birchachhusen pro licencia dt. 3 fl.

fol. $30^{v}$

[1435 II]

(Ca. 1435 Oktober 13) Anno Domini $\mathbf{M}^{\circ} \mathrm{CCCC}^{\circ}$ XXXV circa festum Beati Colomanni martiris assumptus est in decanum facultatis iuris canonici venerabilis et egregius decretorum doctor dominus Johannes Gwerleich, sub quo intitulati et promoti sunt infrascripti:

Mag. Georgius de Herczogburga 3 gr.

Dom. Bohussius de Swola bacc. in artibus 8 gr.

Dom. Martinus de Wolkersdorf 2 gr.

Dom. Nicolaus Bodo 3 sol. d.

5 Dom. Paulus de Apoldia Inferiori presbyter, bacc. in artibus 2 gr.

Johannes Kern de Külsheim bacc. in artibus 2 gr.

Georgius Swentenkrieg de Welss

102 Gesamter Eintrag erst nach Eintragung der „promoti“ an dieser Stelle eingefügt. 
Florianus Im Wald ${ }^{103}$ de Sancto Floriano 6 gr.

Dom. Johannes de Goltperg presbyter Wratislauiensis diocesis 2 gr.

10 Mag. Andreas Labatlan canonicus Strigoniensis 3 sol. d.

Promoti:

Dom. Conradus Reikershofer canonicus Ratisponensis pro doctoratu $1 \mathrm{fl}$.

Dom. Michael plebanus in Stolczemberg de Septemcastris pro doctoratu $1 \mathrm{fl}$.

Dom. Nicolaus Stůbner canonicus Agriensis pro doctoratu $1 \mathrm{fl}$.

Dom. Fredricus Maler alias Sartoris pro baccalariatu et doctoratu $2 \mathrm{fl}$.

15 Dom. Conradus Reikershofer ad structuram facultatis $6 \mathrm{fl}$.

Dom. Michael de Stolczemberg ad structuram facultatis $6 \mathrm{fl}$.

Mag. Michael de Priuidia pro licencia $3 \mathrm{fl}$.

Mag. Anthonius de Schnittauia pro doctoratu $1 \mathrm{fl}$.

Mag. Hartwicus Lampotinger pro baccalariatu $1 \mathrm{fl}$.

fol. $31^{r}$

[1436 I]

(Ca. 1436 April 14) Anno Domini $\mathbf{M}^{\circ} \mathbf{C C C C}^{\circ} \mathbf{X X X V I}^{\circ}$ circa festum Beatorum Tiburcii et Valeriani martirum assumptus est in decanum facultatis iuris canonici venerabilis et egregius decretorum doctor dominus Johannes Polczmacher, sub quo intitulati et promoti sunt infrascripti:

Paulus de Gleybicz bacc. in artibus Cracouiensis 2 gr.

Conradus Velberger de Hallis 2 gr.

Nicolaus Martini de Brůnsperg de Prussia 2 gr.

Johannes de Gürtesch 4 gr.

Promoti:

5 Mag. Johannes de Tapolcza canonicus Waradiensis pro baccalariatu $1 \mathrm{fl}$.

Mag. Caspar Westerndorffer de Lantzhůt pro baccalariatu $1 \mathrm{fl}$.

Dom. Cristannus de Septemcastris pro licencia $3 \mathrm{fl}$.

fol. $31^{v}$

$[1436 \mathrm{II}]$

(Ca. 1436 November 25) Anno Domini Mo CCCC ${ }^{\circ}$ XXXVIo circa festum Beate Katherine, quia precedenti estate propter sevam pestem tunc currentem studium dissolutum fuit, assumptus est in decanum facultatis iuris venerabilis vir magister Conradus de Halstat decretorum doctor, sub quo ${ }^{104}$ intitulati et promoti sunt infrascripti:

103 „Im Wald“ von s. H. mittels Verweiszeichen eingefügt.

104 Von s. H. über der Zeile nachgetragen. 
Dom. Johannes Fëlstein de Bladen prepositus ecclesie Sancte Crucis Wratislauiensis $1 \mathrm{fl}$.

Dom. Vdalricus Nůsdorffer Patauiensis et Ratisponensis ecclesiarum canonicus ${ }^{105}$

Mag. Jeronimus Posser de Salczburga 24 gr. Boh.

Frater Symon de Nidernaltach 8 gr.

5 Jacobus Rosenczweig de Newburga bacc. in artibus 2 gr.

Dom. Erasmus Teisser plebanus in Virgen de Carinthia 4 gr.

Mag. Heinricus de Wyla 6 gr.

Promoti:

Dom. Beringerus de Berlichingen pro licencia $3 \mathrm{fl}$.

Dom. Anthonius de Septemcastris pro licencia $3 \mathrm{fl}$.

10 Mag. Laurentius de Oberngurkch pro licencia $3 \mathrm{fl}$.

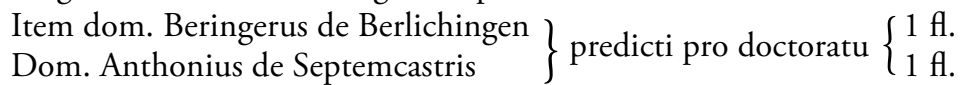

Dom. Cristannus de Septemcastris pro doctoratu $1 \mathrm{fl}$.

Dom. Ladislaus de Strigh archidiaconus ecclesie Transsiluanensis pro doctoratu $1 \mathrm{fl}$.

15 Dom. Johannes Pluemenekcher de Argentina pro baccalariatu $1 \mathrm{fl}$.

Dom. Georgius de Septemcastris pro baccalariatu $1 \mathrm{fl}$.

Dom. Cristannus de Septemcastris pro baccalariatu $1 \mathrm{fl}$.

Dom. Philippus Swarat de Brixina pro baccalariatu $1 \mathrm{fl}$.

fol. $32^{r}$

$[1437 \mathrm{I}]$

(Nach 1437 April 14) Anno Domini millesimo quadringentesimo tricesimo septimo post festum Beatorum Tiburtii et Valeriani martirum assumptus fuit in decanum facultatis iuris canonici venerabilis et egregius decretorum doctor dominus Johannes Seld, in cuius decanatu intitulati sunt infrascripti:

Dom. Erasmus de Reichersperg canonicus regularis $60 \mathrm{~d}$.

Cristoforus Piderman de Wratislauia 4 gr.

Dom. Oswaldus de Rozgon prepositus Agriensis $1 \mathrm{fl}$.

Dom. Johannes de Alba Jula 4 gr.

5 Dom. Thomas Strohofer 6 gr.

Dom. Johannes Schebler 4 gr.

Heinricus Reynwalt bacc. in artibus 1 gr.

Wilibaldus Mayenschein 1 gr.

Blasius de Dworcz 3 gr.

10 Petrus Bartholomei de Go̊rlitz 2 gr.

Petrus Sartoris de Kirchhain 2 gr.

Conradus Speng de Chiczing 4 gr.

105 „24 gr. Boh.“ mittels Zuweisungsstrich diesem und dem folgenden Eintrag zugeordnet. 
Mag. Erhardus de Wolfach 2 gr.

Mag. Jodocus Hawsner de Nouoforo 3 gr.

15 Mag. Johannes Heller de Monaco 4 gr.

Mag. Conradus Pómer de Eystauia 2 gr.

Dom. Bernhardus Lewprechtinger 12 cruciferos

Michael Klingenstainer 2 gr.

Mag. Jeronimus Vogelsang canonicus Olomucensis 2 gr. 30 d. ${ }^{106}$

fol. $32^{v}$

$[1437$ II]

(Nach 1437 Oktober 13) Anno Domini M $^{\circ}$ CCCC $^{\circ}$ XXXVII ${ }^{\circ}$ post festum Beati Colomanni martiris assumptus fuit in decanum facultatis iuris canonici venerabilis et egregius utriusque iuris doctor dominus Johannes de Eych, in cuius decanatu intitulati et promoti sunt infrascripti:

Johannes Hertfelt de Elwangen 2 gr.

Johannes Sutoris de Freynstat presbyter Wratislauiensis diocesis 2 gr.

Conradus Riedrer plebanus in Höflein 4 gr.

Dominicus Hermanni de Slesia 3 gr.

5 Mag. Johannes Lapicide de Kunigshofen 2 gr.

Dom. Michael plebanus in Villa Jacobi de Septemcastris 4 gr.

Dom. Georgius plebanus in Barenghůt in Septemcastris 4 gr.

Mag. Johannes Gůt de Corona plebanus ibidem 3 sol. $10 \mathrm{~d}$.

Dom. Thomas Sybart de Rückpass 2 gr.

10 Dom. Johannes de Schram plebanus in Münsterberg 2 gr.

Valentinus de Mulembach in Septemcastris bacc. in artibus $4 \mathrm{gr}$.

Frater Erhardus de Sancto Egido de Nưrmberga 4 gr.

Dom. Jodocus Seler lic. in artibus 2 gr.

Promoti:

Dom. Wolfgangus Sachs pro baccalariatu $1 \mathrm{fl}$.

15 Dom. Wolfgangus Würm pro baccalariatu $1 \mathrm{fl}$.

$\left.\begin{array}{l}\text { Mag. Gregorius de Valle canonicus Strigoniensis } 1 \mathrm{fl} \cdot{ }^{107} \\ \text { Mag. Nicolaus Lepes archidiaconus ecclesie Transsiluanensis }\end{array}\right\}$ pro baccalariatu1 $\mathrm{fl}$

Dom. Caspar de Sancto Ypolito pro repeticione ad licenciam $3 \mathrm{fl}$.

Dom. Michael plebanus in Villa Thobie pro baccalariatu $1 \mathrm{fl}$.

20 Dom. Johannes Streit de Goltperg pro baccalariatu $1 \mathrm{fl}$.

Dom. Caspar de Sancto Ypolito pro doctoratu $1 \mathrm{fl}$.

Dom. Thomas de Gelestorf pro licencia $3 \mathrm{fl}$.

106 „2 gr.“ und „30 d.“ untereinander auf Rasur nach Tintenwechsel geschrieben.

107 „pro baccalariatu“ mittels Zuweisungslinien diesem und dem folgenden Eintrag zugeordnet. 
fol. $33^{r}$

(1438 Mai 10) Anno Domini M $^{\circ}$ CCCC $^{\circ}$ XXXVIII ${ }^{\circ}$ die Sabbati decima mensis Maii assumptus fuit in decanum facultatis iuris canonici venerabilis et egregius decretorum doctor magister Petrus Pachmulnêr, in cuius decanatu intitulati et promoti sunt infrascripti:

Michael Freyleich 3 gr.

Cristoforus de Rotemburg 2 gr.

Vrbanus Sculteti de Jawr 2 gr.

Wolfgangus de Grèttz 2 gr.

5 Olyuerus de Ymmerzel 2 gr.

Johannes Stůxs de Weyden 2 gr.

Frater Vlricus de Kremsmünster 8 gr.

Dom. Vlricus Ërsinger $1 \mathrm{fl}$. Rh.

Mag. Andreas de Grècz 2 gr.

10 Wolfgangus Freytho̊fl de Gmunden 2 gr.

Promoti:

Andreas de Myskolcz pro baccalariatu $1 \mathrm{fl}$.

Mag. Johannes de Tapoltza pro licencia $3 \mathrm{fl}$.

Dom. Johannes Rül de Frankfordia pro baccalariatu $1 \mathrm{fl}$.

Dom. Johannes Hèz de Brunna pro licencia $3 \mathrm{fl}$.

15 Mag. Vlricus de O̊ringề pro licencia $3 \mathrm{fl}$.

Mag. Hartwicus Lampotinger pro licencia $3 \mathrm{fl}$.

Mag. Johannes de Tapolcza pro doctoratu $1 \mathrm{fl}$.

Mag. Hartwicus Lampotinger pro doctoratu $1 \mathrm{fl}$.

fol. $33^{v}$

[1438 II]

(1438 Oktober 23) Anno Domini $\mathbf{M}^{\circ}$ CCCC $^{\circ}$ XXXVIII feria quinta post ${ }^{108}$ festum Undecim Milium Virginum assumptus est in decanum facultatis iuris canonici venerabilis et egregius vir dominus Martinus de Walthausen, in cuius decanatu intitulati et promoti sunt infrascripti:

Johannes Gelprecht de Lintz 2 gr.

Leonardus Scho̊ntrit de Salczburga 1 gr.

Dom. Castulus Nüsser de Mospurg $60 \mathrm{~d}$.

Dom. Petrus Nafczer de Wysenstaig 3 gr.

5 Johannes Bërtel de Salczbach 2 gr.

108 „post“ über der Zeile eingefügt. 
Bertoldus Ekkinger de Walczhuett 2 gr.

Dom. Johannes Strơmberger plebanus ecclesie Sancte Marie Vallis Lauentine 2 gr.

Dom. Johannes Pencznalber Frisingensis et Augustensis ecclesiarum canonicus

Jodocus Maursteter de Campidona famulus \} 1 fl. Rh.

10 Theobaldus Tưrndl canonicus Patauiensis 3 sol. d.

Dom. Johannes custos Rathibornensis, bacc. in decretis 3 gr.

Mag. Wolfgangus de Knüttefeld 2 gr.

Mag. Johannes Sensensmid 3 gr.

Dom. Heinricus Fleckl prepositus ecclesie Ardacensis 1 fl. Vng.

15 Dom. Johannes Fleckel canonicus Wratislauiensis 3 sol. d.

Bernhardus Baustetter ${ }^{109}$ de Maisemburg 4 gr.

Johannes Stauffer de Plossenstauffen 2 gr.

Promoti:

Dom. Michael de Insula Christiana de ${ }^{110}$ Septemcastris pro baccalariatu $1 \mathrm{fl}$.

Dom. Michael Steger pro baccalariatu $1 \mathrm{fl}$.

20 Dom. Paulus de Gleybitz pro baccalariatu $1 \mathrm{fl}$.

Dom. Bertoldus Gugk pro baccalariatu $1 \mathrm{fl}$.

Dom. Johannes de Albagywla pro baccalariatu obligatur. ${ }^{11}$

fol. $34^{r}$

[1439 I]

(1439 April 14) Anno Domini $\mathbf{M}^{\circ} \mathbf{C C C C}^{\circ} \mathbf{X X X I X}^{\circ}$ in die Beati Tiburtii martiris assumptus fuit in decanum facultatis sacri iuris canonici venerabilis et egregius vir dominus Johannes Polczmacher decretorum doctor, in cuius decanatu intitulati et promoti sunt infrascripti:

Johannes Schaithaimer bacc. in artibus 2 gr.

Petrus Hümel de Petouia 3 gr.

Dom. Johannes canonicus ecclesie Beati Martini in Cepus 2 gr.

Petrus de Poseda 2 gr.

5 Jeronimus Beckensloer de Wratislauia 2 gr.

Mag. Vlricus Horant de Sweinfordia 2 gr.

Paulus Meck de Ornbawr 2 gr.

Dom. Andreas Kürsne̊r de Villseck $30 \mathrm{~d}$.

Mag. Emeramus Parkstainer de Weyden $30 \mathrm{~d}$.

10 Mag. Johannes Hindernpach 2 gr.

109 Mögliche Lesart: „Banstetter“.

110 „Insula Christiana de“ mit anderer Tinte über der Zeile nachgetragen.

111 Zeile mit anderer Tinte nachgetragen. 
Promoti:

Dom. Jacobus Ebser pro licencia $3 \mathrm{fl}$.

Mag. Vlricus Horant pro baccalariatu $1 \mathrm{fl}$.

Dom. Jacobus Ebser pro doctoratu $1 \mathrm{fl}$.

fol. $34^{v}$

[1439 II]

(Nach 1439 Oktober 13) Anno Domini M $^{\circ}$ CCCC $^{\circ}$ XXXVIIII ${ }^{\circ}$ post festum Beati Colomanni martiris assumptus est in decanum facultatis iuris canonici venerabilis et egregius vir dominus Johannes Seld decretorum doctor, in cuius decanatu intitulati et promoti sunt infrascripti:

Franciscus Rewtter de Norrlingen lic. in artibus 2 gr.

Promoti:

Mag. Andreas de Labathlan canonicus Strigoniensis pro baccalariatu $1 \mathrm{fl}$.

Mag. Jeronimus Vogelsangk canonicus Olomucensis pro baccalariatu $2 \mathrm{fl}$. Rh.

Mag. Jeronimus Posser plebanus in Hausdorf pro baccalariatu $1 \mathrm{fl}$.

5 Dom. Thomas canonicus Ardacensis pro baccalariatu $1 \mathrm{fl}$.

Mag. Nicolaus Lepes archidiaconus et canonicus Transsiluanensis pro licencia $3 \mathrm{fl}$.

Mag. Gregorius de Valle Sancti Georgii canonicus Strigoniensis pro licencia $3 \mathrm{fl}$.

Mag. Nicolaus Lepes prescriptus pro doctoratu

Mag. Gregorius prescriptus pro doctoratu

10 Dom. Johannes Plůmenegker ${ }^{112}$ de Argentina pro licencia obligatur. ${ }^{113}$

$[1440 \mathrm{I}]$

(Ca. 1440 April 14) Anno Domini $\mathrm{M}^{\circ} \mathrm{CCCC}^{\circ} \mathrm{XL}^{\circ}$ circa festum Beati Tiburtii martiris assumptus est in decanum facultatis sacri iuris canonici venerabilis et egregius vir dominus Martinus de Walthausen decretorum doctor, in cuius decanatu intitulati et promoti sunt infrascripti:

Dom. Vlricus Prannt canonicus in Sliersee $50 \mathrm{~d}$.

Dom. Wilhelmus Kraft de Vlma bacc. in artibus 3 gr.

Mag. Erhardus Kornmecz de Prugk 4 gr.

Dom. Vlricus Håntinger plebanus in Bischofst(orf) $32 \mathrm{~d}$.

5 Mag. Nicolaus de Glocz bacc. in decretis 3 gr.

Dom. Georgius canonicus ecclesie Baciensis $60 \mathrm{~d}$.

112 Verbessert aus „Plůmengker“, auf Rasur bis „Argentina“.

113 Daneben von a. H.: „habet terminum unius anni“; dies von a. H. gestrichen und ersetzt durch: „in effectu postea persolvit". 
Promoti:

Dom. Erasmus de Reichersperg pro baccalariatu $1 \mathrm{fl}$.

fol. $35^{r}$

Mag. Johannes Gůt de Corona pro baccalariatu $1 \mathrm{fl}$.

Mag. Symon de Asparn pro baccalariatu $1 \mathrm{fl}$.

$10 \mathrm{Mag}$. Jodocus Hawsner pro baccalariatu $1 \mathrm{fl}$.

Dom. Georgius de Septemcastris pro repeticione ad licenciatum $3 \mathrm{fl}$.

[1440 II]

(Ca. 1440 Oktober 13) Anno Domini $\mathbf{M}^{\circ}$ CCCC $^{\circ}$ XL circa festum Beati Colomanni martiris assumptus est in decanum facultatis sacri iuris canonici venerabilis et egregius vir magister Conradus de Halstat decretorum doctor, in cuius decanatu intitulati et promoti sunt infrascripti:

Mag. Johannes Ortlini de Norrlinga 2 gr.

Maternus Tewfel de Brunna 2 gr.

Dom. Johannes Kêser commendator domus Sancti Johannis apud Sanctum Petrum in $\operatorname{Kar}$ [inthia] 6 gr.

Dom. Cristannus de Friberg Brixenensis et Ratisponensis ecclesiarum canonicus fl. Rh.

5 Dom. Petrus Naffczer de Wisenstaig 4 gr.

Johannes Giesser de Monaco bacc. in artibus 4 gr.

Fridericus Flokch de Kornburg bacc. in artibus 3 gr.

Conradus Pruglegker de Welsa bacc. in artibus 3 gr.

Dom. Kaspar plebanus in Preinberg 4 gr.

Promoti:

10 Mag. Symon de Asparn ${ }^{114}$ pro licencia $3 \mathrm{fl}$.

Mag. Andreas de Labatlan ${ }^{115}$ pro licencia $3 \mathrm{fl}$.

Mag. Nicolaus de Glocz pro licencia $3 \mathrm{fl}$.

Dom. Philippus Swarat pro licencia $3 \mathrm{fl}$.

Dom. .. ${ }^{116}$ Lewprechtinger pro baccalariatu $1 \mathrm{fl}$.

15 Dom. Bohussius pro baccalariatu $1 \mathrm{fl}$.

Dom. Johannes Geltprecht de Lynntz pro baccalariatu $1 \mathrm{fl}$.

Mag. Jodocus de Esslinga pro baccalariatu $1 \mathrm{fl}$.

Mag. Georgius de Herczogburg pro baccalariatu $1 \mathrm{fl}$.

114 Ortsangabe mittels Verweiszeichen eingefügt.

115 Ortsangabe mittels Verweiszeichen eingefügt.

116 Zwei Punkte oder „n“. Der Vorname war dem Schreiber offenbar unbekannt. 
fol. $35^{v}$

$[1441 \mathrm{I}]$

(Ca. 1441 April 14) Anno Domini $\mathbf{M}^{\circ} \mathbf{C C C C}^{\circ} \mathbf{X L I}^{\circ}$ circa festum Beati Tyburtii martiris assumptus est in decanum facultatis iuris canonici venerabilis et egregius vir dominus Johannes Polczmacher decretorum doctor, in cuius decanatu intitulati et promoti sunt infrascripti:

Intitulati:

Erhardus de Asparn Mayori bacc. in artibus 4 gr.

Vlricus Kaltemprunner de Tegernsee 4 gr.

Heinricus Pawr de Nurmberga 2 gr.

Mag. Leonhardus de Stain Carniole 2 gr.

5 Mag. Georgius Schrekch de Schărding 2 gr.

Mag. Nicolaus Satler de Kunigsberg 2 gr.

Matheus Andree de Lyppa 2 gr.

Dom. Johannes Schachner professus monasterii Sancti Lamperti 4 gr.

Johannes Gruèn de Hallis 2 gr.

10 Mag. Johannes de Pankotha 2 gr.

Promoti:

Dom. Johannes Pêrtl de Sulczpach pro baccalariatu $1 \mathrm{fl}$.

Dom. Petrus Nafczer de Wysenstaig pro baccalariatu $1 \mathrm{fl}$.

Dom. Vdalricus de Kremsmünster pro baccalariatu $1 \mathrm{fl}$.

[1441 II]

(Ca. 1441 Oktober 13) Anno Domini M $^{\circ}$ CCCC $^{\circ}$ XLI $^{\circ}$ circa festum Beati Colomanni martiris assumptus est in decanum facultatis iuris canonici venerabilis et egregius vir magister Petrus Pachmúlner decretorum doctor, in cuius decanatu intitulati et promoti sunt infrascripti:

Dom. Lampertus de Rukhendorf dt. unum fl. Rh.

Dom. Wolfgangus de Tẻmsweg dt. 4 gr.

Dom. Johannes Pawswågl de Olomutz dt. 4 gr.

Dom. Johannes Ehinger de Landshůta dt. 2 gr.

Promoti:

5 Dom. Johannes de Franckfordia ad licentiam $3 \mathrm{fl}$.

Dom. prepositus Sancte Crucis Wratislauiensis Johannes de Bladen pro baccalariatu $1 \mathrm{fl}$.

Mag. Erhardus de Wolfach pro baccalariatu $1 \mathrm{fl}$. 
Dom. Andreas Kürsner de Villsegk pro baccalariatu $1 \mathrm{fl}$.

fol. $36^{r}$

[1442 I]

(Ca. 1442 April 14) Anno Domini Mo CCCC $^{\circ}$ XLII $^{\circ}$ circa festum Beati Tyburcii martiris assumptus est in decanum facultatis iuris canonici venerabilis et egregius decretorum doctor dominus Martinus de Walthausen, in cuius decanatu intitulati et promoti sunt infrascripti:

Intitulati:

Dom. Gabriel canonicus regularis de Wratislauia 4 gr.

Dom. Johannes de Gotwico quinque gr.

Dom. Martinus plebanus in Pòlan 4 gr.

Egidius Scherffemberg de Lůbschütz 2 gr.

5 Erasmus Kirchhof de Goth 1 gr.

Johannes de Ringenstain 2 gr.

Mag. Gregorius Andree de Molhausen 2 gr.

Promoti:

Dom. Paulus de Gleybitz ad licenciam $3 \mathrm{fl}$.

Dom. Symon de Nydernaltach ad licenciam $3 \mathrm{fl}$.

10 Mag. Jeronimus Posser de Salcz[burga] ad licenciam $3 \mathrm{fl}$.

Dom. Jacobus Rosenczwey de Weyssemburga ad licenciam $3 \mathrm{fl}$.

Dom. Bertoldus Gugk ad licenciam $3 \mathrm{fl}$.

Dom. Johannes canonicus Czepusiensis pro baccalariatu $1 \mathrm{fl}$.

Dom. Vrbanus de Jawr pro baccalariatu $1 \mathrm{fl}$.

15 Mag. Ludouicus Leupolt de Monaco pro baccalariatu $1 \mathrm{fl}$. 
Register 



\section{Vorbemerkung}

Die Personen sind im Personenregister nach den Vornamen, innerhalb desselben Vornamens nach dem Zunamen oder dem Namen des Herkunftsortes alphabetisch geordnet. Als Muster für die alphabetische Reihung dienten das Repertorium Germanicum sowie Paul Uibleins Editionen der Acta Facultatis Artium und der Acta Facultatis Theologiae. $\mathrm{C}, \mathrm{CH}$ und $\mathrm{K}, \mathrm{F}$ und $\mathrm{V}, \mathrm{I}$, J und $\mathrm{Y}$ sowie $\mathrm{Z}$ und $\mathrm{CZ}$ sind im Anlaut als je ein Buchstabe vereint, nicht jedoch im Wortinneren. In der alphabetischen Reihenfolge wurde å und è unterschiedlich behandelt.

Im Register der $\mathrm{Zu}$ - und Ortsnamen sind die Ortsnamen in moderner Form wiedergegeben. Von den in der Originalquelle vorkommenden Ortsnamen wird auf die moderne Namensform verwiesen. Nennungen der originalen Ortsnamen erfolgen nur dann nicht, wenn sie vom modernen Ortsnamen nicht weiter als zwei Eintragungen entfernt stehen. Dadurch sollte eine allzu intensive Überfrachtung des Registers der Zuund Ortsnamen verhindert werden. Unter den $\mathrm{Zu}$ - bzw. Ortsnamen erscheinen alle in der Juristenmatrikel vorkommenden Personen mit ihrem Vornamen.

Das Register verweist sowohl auf die vorangehende Textedition der Wiener Juristenmatrikel als auch auf die nachfolgenden gedruckten Quellen aus dem Archiv der Universität Wien:

Gall MUW I (Die Matrikel der Universität Wien, 1. Band: 1377-1450, bearb. von Franz GALL etc., Graz/Köln 1956);

Schrauf, AFM (Acta Facultatis Medicae Universitatis Vindobonensis, Band 1-2, hrsg. von Karl SCHRAUF, Wien 1894-1899);

Schrauf, NH (Die Matrikel der Ungarischen Nation an der Wiener Universität 14531630, hrsg. von Karl SCHRAUF, Wien 1902);

Uiblein, AFA I (Acta Facultatis Artium Universitatis Vindobonensis 1385-1416, hrsg. von Paul UIBLEIN, Publikationen des Instituts für Österreichische Geschichtsforschung, VI. Reihe: Quellen zur Geschichte der Universität Wien, 2. Abt., Graz/Köln/ Wien 1968);

Uiblein, AFTh (Die Akten der Theologischen Fakultät der Universität Wien 13961502, 2 Bände, hrsg. von Paul UIBLEIN, Wien, 2. Aufl. 1995);

Weiters wurden auch Hinweise auf Quellenbelege aus dem Band „Uiblein, Mittelalterliches Studium“ aufgenommen (Paul Uiblein, Mittelalterliches Studium an der Wiener Artistenfakultät. Kommentar zu den Acta Facultatis Artium Universitatis Vindobonensis 1385-1416, Schriftenreihe des Universitätsarchivs, Universität Wien, Band 4, Wien, 2. Aufl. 1995). 



\section{Register der Vornamen}

$-\mathrm{A}-$

Achacius

- de Tyrna 1406 II 4. MUW 1, 1400 I A 12 (Ach. de Tiernauia)

Adolfus (Adolffus)

- de Eppenstain Colonensis et Treuerensis canonicus, archidiaconus Treuerensis 1424 I 19. MUW 1, 1420 II R 2 (Alolfus de Eppenstayn baro, can. et archidiac. eccl. Treuerensis)

Albertus

- de Byla 1408 I 6. MUW 1, 1402 I R 7 (Alb. Merklinger de Weila) oder 1402 I R 71 (Alb. de Weyla); Uiblein, AFA I, 496 (Alb. de Wila)

- de Borzislauicze Cracouiensis et Bosnonensis canonicus 1414 I 10. MUW 1, 1414 I H 3 (Alb. de Worsislawicze)

- de Keczkemeth 1426 II 13. MUW 1, 1420 I H 29 (Alb. Jacobi de Kicschkamet)

- de Kerot familiaris eiusdem prepositi (sc. Ladislaus de Marcellis) 1419 I 10. MUW?

- de O̊tingen (comes) 1432 II 1. MUW 1, 1432 II R 1 (Alb. comes de Ötting, can. eccl. Argentinensis)

- de Potendorff 1419 II 12. MUW 1, 1413 I A 1 (Alb. Potendorffer); Uiblein, AFA I, 496; Uiblein, Mittelalterliches Studium, 119

- Slewser (als b. a.) 1429 I 11. MUW 1, 1425 II H 43 (Alb. Slewsser de Hainouia)

- de Waradino (de Baradino) (als lic. a.) $1428 \mathrm{I} 3$; bacc. in decr. (als m. a.) 1430 I 23; lic. in decr. (archidiac. et canon. eccl. Strigoniensis) 1432 II 11; dr. decr. 1434 II 16. MUW 1, 1422 I H 36 (Alb. de Baradino)

Alexius (Allexius)
- Strenberger 1429 I 9. MUW 1, 1426 II R 1 (Alex. Stremberger)

Alfonsus (Alfansus)

- Gamezii (Gomezii) canonicus Toletanus 1424 II 5; bacc. in decr. 1428 II 14; lic. in decr. 1430 I 23; dr. decr. 1430 I 24. MUW 1, 1424 II R 28 (Alf. Comecii)

Ambrosius

- Neczowencz de Golaw 1417 I 16. MUW 1, 1415 I S 10

Andreas

- canonicus Agriensis 1419 II 6; bacc. in decr. 1423 I 29; lic. in decr. 1427 II 11. MUW?

- de Astershofen plebanus in Smaichingen 1413 I 7. MUW?

- Burmeyster de Gribiswaldis 1416 I 13. MUW 1, 1416 I S 1 (Andr. Burmester)

- Chlosner de Geren 1415 II 1. MUW?

- Kürsnear de Villseck (Villsegk) 1439 I 8; bacc. in decr. 1441 II 8. MUW 1, 1439 I R 14 (Andr. Kürsner de Villsegk)

- de Falkenberg plebanus in Wiselburg 1413 I 5. MUW 1, 1411 I A 28 (Andr. de Valkenberg)

- Feynockh de Berchtoltstorf (als m. a.) 1432 I 8. MUW 1, 1425 I A 21 (Andr. Veifach de Percheltsdorff?)

- de Greàcz (als m. a.) 1438 I 9. MUW 1, 1432 II A 8 (Andr. Aspach) oder 1433 II A 2 (Andr. Fabri)

- Labatlan (Labathlan) canonicus Strigoniensis (als m. a.) 1435 II 10; bacc. in decr. 1439 II 2; lic. in decr. 1440 II 11. MUW 1, 1428 II H 17 (Andr. de Labatlan)

- de Laybaco (als m. a.) 1431 I 5. 
MUW 11418 II $^{1} \mathrm{H} 6$

- Langkhaim de Prussia 1428 II 2. MUW 1428 II S1 (Andr. Lankhaim plebanus in Bern)

- Mawser (als m. a.) 1416 II 10. MUW 1, 1398 II A 38 (Andr. Müser); Uiblein, AFA I, 498 (Andr. Mauser); Uiblein, Mittelalterliches Studium, 119; Uiblein AFTh 2, 623 (Andr. Mauser [de Wienna])

- Michaelis de Wratislauia bacc. in decr. 1425 II 5. MUW?

- Michaelis de Zerblin 1421 I 23. MUW 1, 1421 I H 42 (Andr. Michaelis de Serbin)

- de Myskolcz (Myschkolcz) 1434 II 5; bacc. in decr. 1438 I 11. MUW 1, 1424 II H 21 (Andr. Sartoris de Miskolcz)

- Nas canonicus Wissegradensis 1412 II 13. MUW 1, 1412 II H 19 (Andr. Nas de Montibus Chuttinis, can. Bischegredensis)

- canonicus monasterii Neunburgensis 1432 I 5. MUW?

- archidiaconus et canonicus Olomucensis 1412 II 6. MUW 1, 1412 II H 16

- Pfaffendorff canonicus ecclesie Sambiensis Ordinis Tewtonicorum 1417 I 6. MUW 1, 1417 I S 2

- Putnyt de Aw 1418 I 11. MUW 1, 1418 I R 28 (Andr. Puttnyt de Awe)

- plebanus in Redsch 1430 II 20; identisch mit Andreas plebanus in Recz de Septemcastris bacc. in decr. 1433 II 7? MUW?

- canonicus de Sancto Yppolito 1416 II 4. MUW 1, 1412 I A 2 (Andr. can. regularis apud Sanctum Ypolitum)

- Santperg (als m. a.) 1428 II 5. MUW 1, $1419 \mathrm{II}^{2}$, S 4 (Andr. de Hamerstain?)
- Schoonaw plebanus in Stubelaw 1424 I 2. MUW 1, 1424 I S 2 (Andr. Schonaw de Prussia)

- de Sittauia 1417 I 5. MUW 1, 1417 I H 5 (Andr. de Zwittauia)

- de Wittemberg 1416 II 22, bacc. in decr. 1421 I 22. MUW 1, 1416 II S 4 (Andr. de Wittenberg)

Antonius (Anthonius)

- de Busa canonicus ecclesie Sancti Thome in Strigonio 1431 II 3. MUW 1, 1431 II H 23 (Anth. Stephani de Wusa)

- Füchtensteiner 1413 II 5. MUW 1, 1412 II A 20 (Ant. Fewchtenstainer de Wyenna)

- plebanus in Mulenbach 1422 I 21; bacc. in decr. 1425 II 4; lic. in decr. 1427 II 12; dr. decr. 1428 I 11. MUW 1, 1399 II H 8 (Ant. de Mülnbach)

- de Muschna (als lic. a.) 1427 II 6. MUW 1, 1423 I H 24

- plebanus in Omlaschz (Hamlach) (de Septemcastris) 1430 II 16; bacc. in decr. 1432 II 9; lic. in decr. 1436 II 9; dr. decr. 1436 II 12. MUW?

- de Schnitauia (Schnittauia, Sintauia) (als m. a.) 1430 I 7; bacc. in decr. 1433 I 13; lic. in decr. 1435 I 14; dr. decr. 1435 II 18. MUW 1, 1425 II H 11 (Ant. Stephani de Sinthauia)

Arbogastus

- Elhart (als m. a.) 1433 II 3; bacc. in decr. 1434 I 16. MUW 1, 1422 I R 72 (Arbog. Elhart de Argentina)

Arnoldus

- de Lawtershaym plebanus ibidem 1415 I 15. MUW 1, 1413 II R 59 (Arn. de Lutersheim); Uiblein, AFA I, 499 (Arn. de Lutershein [Lautersheim]) 
Barnabas

- de Mera 1431 I 10. MUW 1, 1422 I H 32

- de Tyssawassan (Tysswassan, Tyzzawazzan) (als m. a.) 1418 I 9; bacc. in decr. $1421 \mathrm{I} 21$; lic. in decr. und dr. decr. 1422 I 28. MUW 1, 1412 II H 11 (Barbanas[sic!] de Tissafaschan?); Uiblein, AFA I, 499 (Barn. de Harssanbassan)

Bartholomeus (Bartolomeus)

- in Altzuna 1406 II 14. MUW 1, 1406 II H 12 (Barth. Alcznohet); Uiblein, AFA I, 499 (Barth. plebanus in Septemcastris)

- Kren de Erdpurg 1410 I 5. MUW 1, 1409 I A 13 (Barth. Egidii [de] Erdburg)

- Glawbis 1416 II 16. MUW 1, 1416 II H 29 (Barth. Glambis)

- de Martpurga 1426 II 8 . MUW 1, 1422 II A 12 (Barth. de Margburga)

- Menseer plebanus in Hymperg 1423 I 7. MUW 1, 1424 I A 22 (Barth. Mènseer de Frankenmarcht intytulatus sub Mag. Cholomanno Cholb anno etc. 97 et ibi sub nacione Renensium)

- Sauigerue prepositus ecclesie kathedralis Tarbatensis 1423 I 23. MUW 1, 1423 II S 1 (Barth. Zauigerne)

- Czedlicz de Hiersberg 1423 II 7. MUW 1, 1419 II $^{2}$ H 16 (Barth. Zedlicz de Hirsberg)

Benedictus

- de Bathor baro 1418 II 15. MUW 1, 1417 I H 46 (Bened. filius Johannis de Bator)

- de Waradino (als m. a.) 1422 II 11. MUW1, 1416 II H 15 (Bened. de Wardin?)

- de Worach (als m. a.) 1418 I 7. MUW 1, 1413 II H 53 (Bened. de Buroth); Uiblein, AFA I, 500 (Bened. de Poroch)
Berengarus (Beringerus, Berngerus)

- de Berlichingen lic. in decr. 1436 II 8; dr.decr. 1436 II 11. MUW?

Bernhardus (Wernhardus)

- Baustetter de Maisemburg 1438 II 16. MUW 1, 1438 II R 77 (Bernh. Wüstetter canonicus ecclesie Jerosolimitane)

- Kugelrềwter 1418 II 29. MUW?

- Lang canonicus Sancti Georii in castro Gnesensi 1419 I 11. MUW 1, 1414 I H 36 (Bernh. Lang de Cracouia)

- Lewprechtinger 1437 I 17; bacc. in decr. 1440 II 14?. MUW 1, 1437 II R 37 (Leonhardus Leoprechtinger can. regularis?)

- Nebraw (als m. a.) 1414 I 25. MUW 1,1414 I S 22

- cappelanus in curia Newnburgensi 1432 I 6. MUW 1, 1432 I A 24 (Bernh. Pawr de Ort)

- de Raczenhausen canonicus Basiliensis 1412 I 22. MUW 1, 1412 I R 5 (Bernh. de Roczenhausen dictus vom Stein, can. Bisliliensis et sancti Theodardi)

- frater Ordinis Sancti Johannis1427 I 4. MUW?

- de Sindolfing 1417 I 7. MUW 1, 1413 I R 3 (Bernh. de Sindolfingen); Uiblein, AFA I, 500 (Bernh. de Sindelfingen)

Bertholdus (Bertoldus)

- Ekkinger de Walczhuett 1438 II 6. MUW1, 1430 II A 15

- Gugk bacc. in decr. 1438 II 21; lic. in decr. 1442 I 12. MUW 1, 1432 II R 11 (Bert. Gukch de Mittenwald)

- de Helta (als lic. a.) 1415 II 11. MUW 1, 1408 I H 8 (Bartholomeus de Helta); Uiblein, AFA I, 499 (Bartholomeus de Helta)

- Sesselman 1415 I 22. MUW 1, 
1412 I R 83 (Bercht. Sesselman de Culmbach)

- Tebershaymer canonicus ecclesie Sancti Johannis in Håwgis 1425 I 5. MUW 1, 1425 I R 30 (Berchtoldus Theberschaymer studens in jure)

Blasius

- Chanadiensis cappellanus predicti prepositi (sc. Ladislaus de Marcellis) 1419 I 9. MUW 1, 1419 I H 22 (Blas. de Schanadino de Theotonica) - de Dworcz 1437 I 9. MUW?

Bohuslaus (Bohoslaus, Bohussius)

- plebanus de Bano 1423 II 1; bacc. in decr. 1426 II 21. MUW 1, 1423 I H 25 (Bohosl. plebanus in Wano)
- de Lascho 1421 I 13. MUW 1, 1415 II H 33 (Bohusl. de Lasskow)

- de Swola (als bacc. a.) 1435 II 2; bacc. in decr. 1440 II 15. MUW 1 , 1431 II H 26 (Bohoslaus Petri de Zwola: S. 177, Anm. 3: Von späterer Hand: episcopus Olomuczensis)

Briccius

- de Buda 1417 I 3. MUW 1, 1412 I $\mathrm{H}$ 12; Uiblein, AFA I, 501; Uiblein, Mittelalterliches Studium, 120

Bruno

- Trudelonis de Ytstein 1417 I 15. MUW 1, 1416 II R 21 (Wruno Trudelonis de Itstein)
Caspar (Kaspar)

- de Brezewicz Sepusiensis 1426 II 11. MUW?

- Kalbskoph plebanus in Kunigsteten 1425 I 1. MUW 1, 1411 II A 5

(Kasp. Chalbskoph de Wels)

- Ebenhawser canonicus ecclesie Pawburgensis 1428 II 10; bacc. in decr. 1431 II 23. MUW 1, 1428 II R 46 (Casp. de Pawburga)

- plebanus in Veklaprugk 1434 I 11. MUW 1, 1434 II R 3 (Casp. Hornperger)

- Fridwalt de Nissa 1416 I 23. MUW 1, 1415 II H 32

- Herr de Vlma 1411 II 11. MUW 1, 1411 I R 106

- (de) Mayselstain (Mayslstain) dr. decr. 1405 II 2 (1405 Dezember 1); jur. Dekan 1408 I, 1409 II, 1414 I, 1416 I, 1419 II, 1424 I. MUW 1, 1385 II A 23 (Casp. Maisselstain); Uiblein, AFA I, 502 (Casp. Maiselstein); Uiblein, Mittelalterliches Studium, 120

- Mendorfer 1402 I 5. MUW 1, 1401 II R 45 (Casp. Mendorffer)
- de Piburga (Westerndorffer de Lantzhout) (als m. a.) 1433 II 16; bacc. in decr. 1436 I 6. MUW 1 , 1429 I R 7 (Casp. Bestendorffer de Pyburg)

- plebanus in Preinberg 1440 II 9. MUW?

- canonicus regularis ad Sanctum Ypolitum (de Sancto Ypolito) 1432 I 2; bacc. in decr. 1435 I 9; lic. in decr. 1437 II 18; dr. decr. 1437 II 21. MUW 1, 1432 I A 18 (Casp. Veirtager)

- Sigersdorffer 1424 II 3. MUW 1, 1423 I A 28 (Casp. de Sygesdorff)

- canonicus ecclesie Strigoniensis bacc. in decr. 1430 II 25. MUW?

- de Wolffsperg bacc. in decr. 1427 II 15. MUW?

- Czener (de Patauia) 1431 I 6; bacc. in decr. 1434 II 11. MUW 1, 1431 I R 14

Castulus

- Nüsser de Mospurg 1438 II 3. MUW 1, 1437 II R 73

Cyriacus (Ciriacus)

- Knot canonicus ecclesie Eystetensis 
1423 I 18. MUW 1, 1423 I R 152 (Cyr. Knott)

- de Quinque [Ecclesiis] 1429 I 7 (als m. a.). MUW?

Clemens

- de Agria 1411 II 15. MUW 1, 1401 I H 3 (Clem. presbyter, rector altaris sancte Trinitatis in ecclesia Agriensi, protunc procurator eiusdem); Uiblein, AFA I, 503

- Almår de Salzpurga 1415 I 8. MUW 1, 1413 II R 49? (Clem. de Bongaw)

- plebanus ecclesie in Dobrica 1431 II 13. MUW?

- Drzewiczky 1418 I 21. MUW 1, 1418 I H 35 (Clem. de Dreswiczken)

Colomannus (Colmannus, Cholomannus)

- (Knapp de Hyppleins) canonicus Newmburgensis 1422 I 1 (als b. a.); bacc. in decr. 1425 II 7; lic. in decr. 1428 II 12; dr. decr. 1430 II 33. MUW1, 1419 II 2 A 2 (Colom. Cnapp de Hipples)

Conradus (Chunradus)

- Arnoldi canonicus ecclesie Sancti Stephani Maguntinensis 1430 I 20. MUW 1, 1430 I R 2

- (Bladeck, Bladek de Wienna) 1420 I 3 (als m. a.); bacc. in decr. 1424 II 10; lic. in decr. 1426 I 10; jur. Dekan 1427 I. MUW 1, 1413 I A 16 (Conr. Pladeck)

- de Campidena 1422 I 22. MUW 1, 1422 I R 69 (Conr. de Ca[m]pidona)

- de Chreig (Kreyg) prepositus in Volkenmarckt 1431 II 15 (in diesem Semester keine Taxe bezahlt); 1432 I 1. MUW?

- Kuenlein (Künlein) de Dyppurg (Dyeppurg) 1429 II 4; bacc. in decr. 1431 II 24. MUW 1, 1423 I R 72 (Conr. Kunlein de Diepurg)

- Eberhardi de Hallis 1410 I 10. MUW 1, 1410 I R 66

- de Elbang 1423 I 2; bacc. in decr. 1426 II 20. MUW 1, 1420 II R 19
(Conr. Pfalhaimer)

- Emmen de Hildeshaym 1430 II 21. MUW 1, 1424 I S 5 (Conr. Emenn de Hildishaim)

- de Varembach 1413 I 22. MUW 1, 1412 II R 17 (Conr. de Farenpach)

- Velberger de Hallis 1436 I 2. MUW 1, 1434 II R 27 (Conr. de Hallis)

- de Halstat (Hallstat) 1414 II 10 (als m. a.); bacc. in decr. 1419 I 3; lic. in decr. und dr. decr. 1422 I 23; jur. Dekan 1423 I, 1427 II, 1429 II ,1434 I, 1436 II, 1440 II. MUW 1, 1411 II A 34 (Conr. Pistoris de Hallstat); Uiblein, AFA I, 504f.; Uiblein, Mittelalterliches Studium, 120; Schrauf, AFM 1, 104 (Mag. Conr. de Hallstat, decretor. doctor; Schrauf, AFM 2, 251 (Mag. Conr. de Halstat, rector. univ.)

- de Helmstat 1409 I 1. MUW 1, 1409 I R 48

- (Henrici) de Hildesheim (de Hildensem) 1407 II 2; lic. in decr. 1414 I 31 (als m. a.). MUW 1, 1407 II S 2 (Chunr. Heinrici [de] Heidelshein); Uiblein, AFTh 2, 449, Anm. 381

- Herbeyn de Tywing 1426 I 5. MUW?

- de Langstat 1422 II 3 (als m. a.); bacc. in decr. 1424 II 14. MUW 1, 1415 I R 49

- Pachenstain 1429 II 15 (als m. a.). MUW 1, 1423 II R 34 (Conr. de Bakchenstain)

- de Pemeshaim 1430 I 18. MUW 1, 1412 II R 60 (Conr. Probst de Penshem)

- Phörtner de Jukarim 1412 I 6. MUW 1, 1410 I S 4 (Conr. Phertner de Jocarim); Uiblein, AFA I, 505 (Conr. de Jukerim)

- Pòmer de Eystauia 1437 I 16 (als m. a.). MUW?

- de Poppenhaim marscalcus 1413 I 32. MUW 1, 1412 II R 36 (Conr. Marschalich, can. Astauiensis)

- Pruglegker de Welsa 1440 II 8 
(als b. a.). MUW 1, 1438 II R 120? (Conr. Prugklekker de Patauia)

- Râwching (Reweining, Ruyhing) (de Freyburg) 1411 II 20; bacc. in decr. 1416 I 30; lic. in decr. 1416 II 35; jur. Dekan 1417 I. MUW 1, 1404 I A 21; Uiblein, AFA I, 504 (Conr. de Friburgo); Schrauf, AFM 1, 106 (Conr. Ròchling, lic. iur.)

- Reykershouer (Reykershofer, Reikershofer, Reickershofer) Ratispanensis et Eystetensis ecclesiarum canonicus 1430 II 22; bacc. in decr. 1434 I 19; lic. in decr. 1435 I 11; dr. decr. 1435 II 11; bezahlt 6 fl. ad structuram facultatis $1435 \mathrm{II}$ 15. MUW 1, 1431 I R 51 (Conr. Beykershofer[sic!], can. eccl. Ratisponensis)

- Reintaler de Oberberga 1426 II 10. MUW?

- Reyndel de Fredeberg 1422 II 9. MUW 1, 1420 II R 4 (Conr. de Fridberg)

- Reysperger plebanus in Monte Sancti Viti 1421 II 3. MUW?

- Riedrer plebanus in Höflein 1437 II 3. MUW?

- de Salczburga monachus monasterii in Altach 1411 II 2. MUW 1, 1411 II R 3 (Conr. de Salczburga, professus OSB in Altach inferiori)

- Scharb de Lawben 1412 II 20. MUW 1, 1412 II R 75 (Conr. Schaib de Lauben)

- Schedlein 1416 II 20. MUW?

- Schenkch de Külsheim 1418 II 17 (als m. a.). MUW 1, 1412 I R 14. Uiblein, AFA I, 506 (Conr. Schenck de Kulshein)

- Schober de Hasfordia 1416 II 34. MUW 1, 1411 I R 46 (Conr. de Hasfordia)

- Schürg de Tranbach 1413 I 23. MUW 1, 1413 I R 35 (Conr. Schurich de Kranach?)

- Seczel de Rütlinga 1416 II 25. MUW
1, 1410 II R 3 (Conr. Scheczel de Rutlinga); Uiblein, AFA I, 506 (Conr. de Rütling[a]); Uiblein, Mittelalterliches Studium, 120

- Seglauer (Zyglawer) 1402 I 3 (als m. a.); jur. Dekan 1413 II. MUW 1, 1390 I R 25 (Conr. Segglauer); Uiblein, AFA I, 506; Uiblein, Mittelalterliches Studium, 120

- Seybolt de Memmingen 1418 I 23. MUW 1, 1409 I R 21 (Chünr. Sybolt); Uiblein, AFA I, 506 (Conr. Sybolt [Sywolt])

- Seydenliep de Erfordia 1430 I 2. MUW 1, 1427 I R 5 (Conr. Seydenlieb)

- Sybmer bacc. in decr. 1435 I 7. MUW 1, 1423 I R 13? (Conr. Sibenhờ de Eschenbach)

- Speng de Chiczing 1437 I 12. MUW 1, 1435 II R 76 (Conr. Speng de Kiczing)

- Trawner 1406 II 1. MUW 1, 1403 I A 26 (Conr. Travner); Uiblein, AFA I, 507 (Conr. Träner)

- de Vlma 1427 II 8 (als b. a.); bacc. in decr. 1431 I 15. MUW 1, 1423 II R 8? (Conr. Mutscheller de Vlma) oder 1427 I R 54? (Conr. Gessler de Vlma)

- professus monasterii Wymbergensis 1420 I 15. MUW?

- Wirsung de Lantzhuta 1411 I 10. MUW 1, 1411 I R 54 (Conr. Wirsungen de Lantzhuta); Uiblein, AFA I, 505 (Conr. de Lanczhůt)

- Wolff de Wartemberg 1419 II 5; bacc. in decr. 1422 I 10. MUW 1 , 1419 II $^{2} \mathrm{H} 6$ (Conr. Wolf de Vartenberg)

- Zeydlareảr de Monaco 1410 II 2. MUW 1, 1407 I R 25; Uiblein, AFA I, 505 (Conr. de Monaco); Uiblein, Mittelalterliches Studium, 120

Krafto

- de Swarczach 1412 II 3 (als m. a.); dr. decr. 1415 I 14. MUW 1, 1403 I 
R 7 (Crafto de Swarczach); Uiblein, AFA I, 507 (Crafto de Nüerenberga [de Swarzach]); Uiblein, Mittelalterliches Studium, 121

Christannus (Cristannus, Cristamus)

- de Brunna 1409 I 4. MUW 1, 1409 I H 9 (Crist. Körbler de Brunna)

- Ewart de Stolczemberg 1434 I 5. MUW 1, 1434 I H 20

- plebanus in Villa Hainperti 1430 II 15; bacc. in decr. 1433 II 8; lic. in decr. 1436 I 7; dr. decr. 1436 II 13. MUW 1, 1430 II H 31 (Crist. plebanus in Villa Humperti)

- de Friberg Brixenensis et Ratisponensis ecclesiarum canonicus 1440 II 4. MUW 1, 1440 II R 75 (Crist. de Freyberg)

- Håppel plebanus in Puech 1420 I 1. MUW 1, 1420 I R 5 (Crist. Heppel, plebanus in Puch Frisingensis diocesis)

- de Hiltenstorff de Wurcia 1429 II 20. MUW 1, 1429 II H 39 (Crist. plebanus in Hildelstorff)

- de Molinpach 1432 II 4. MUW 1, 1428 II H 10 (Crist. Currificis de Múlenbach)

- Mulpacher de Kufstain 1426 I 6. MUW 1, 1423 I R 23 (Crist. Mulbacher de Kufsteyn)

- Murher de Grassertal 1412 I 5. MUW?
- de Reddino 1420 II 8. MUW 1, 1419 I S 7 (Crist. Johannis de Reddin)

- de Septemcastris 1409 II 4 (als m. a.); bacc. in decr. 1436 II 17. MUW 1, 1392 II H 7; Uiblein, AFA I, 508

- de Wasserburg 1424 I 18 (als m. a.). MUW 1, 1409 I R 31? (Crist. Oder de Wasserburg) oder 1409 I R 78? (Crist. de Wasserburga); Uiblein, AFA I, 508 (Crist. de Wasserburga)

- Wurm de Salina 1412 I 20. MUW 1, 1412 I R 105

Christoferus (Cristofferus, Cristoforus)

- Arnolt de Tullaco 1429 II 12. MUW 1, 1425 I A 31 (Cristof. Arnold de Toblaco)

- Dachsawer 1434 II 3. MUW 1, 1429 II R 58 (Cristof. Dachsawer de Eberspewnt)

- Guldein (de Rain) 1423 I 24 (als m. a.); bacc. in decr. 1426 II 19. MUW?

- Lusning plebanus in Strasgang 1422 I 11. MUW?

- de Milaslauia 1420 II 3. MUW?

- Piderman de Wratislauia 1437 I 2. MUW 1, 1433 II H 12 (Cristof. de Wratislauia)

- de Rotemburg 1438 I 2. MUW 1, 1437 I H3

- Welczer 1420 I 9. MUW 1, 1416 I A 5? (Cristophor Welser, plebanus Sancte Marie)
David

- de Gyrold famulus domini archidiaconi (sc. Martinus archidiaconus Transsiluaniensis) 1428 II 9. MUW 1, 1428 II H 31? (Dauid Martini de Syrolth)

- Jung 1414 I 26. MUW 1, 1414 I S 23 (Dauid Jung de Gdanczk)

Demetrius

- de Gich canonicus ecclesie Herbipo- lensis 1414 II 2. MUW 1, 1414 II R 12 (Demetr. de Gich, can. et archydiac. eccl. Herbipolensis)

- de Czerod 1412 II 17. MUW 1, 1412 II H 23 (Demetr. de Czeiod)

- Czuporis archidiaconus de Kykuw, canonicus in ecclesia Transsiluana 1429 I 6. MUW 1, 1425 I H 30 (Demetr. Czupur, nobilis de Monoslow) 
Dionisius (Dyonisius)

- Jax custos et canonicus ecclesie Transsiluane 1414 I 24. MUW?

- de Newburga 1426 II 4 (als m. a.). MUW?
Dominicus

- Hermanni de Slesia 1437 II 4. MUW 1, 1437 II H 3

- de Mo̊lnar (Möner) 1424 I 11; bacc. in decr. 1428 I 13. MUW 1, 1421 I H 19 (Domin. de Molnar)
Eberhardus

- Molitoris de Nuremberga 1415 I 12. MUW 1, 1412 II R 6

Egidius

- Scherffemberg de Lůbschùtz 1442 I 4. MUW 1, 1434 II H 7

Eglolfus

- prepositus Ardacensis 1406 II 6. MUW 1, 1399 II A 14? (Egloffus Futbolt de Rüth). Uiblein, AFA I, 509

- Etterli de Bringk in Ergaw 1412 II 10. MUW 1, 1410 I A 11 (Eglinus Ortlini de Brugk); Uiblein, AFA I, 509 (Eglolf. de Pruka)

Emeramus

- Parkstainer de Weyden 1439 I 9 (als m. a.). MUW 1, 1434 II R 7 (Emerammus Pachstainer de Weyden)

\section{Emericus}

- custos et canonicus ecclesie Baciensis 1430 II 13 (als m. a.). MUW 1, 1425 I H 19? (Emericus de Kekek)

Erasmus

- Kemereàr 1412 I 12. MUW?

- Kirchhof de Goth 1442 I 5. MUW?

- Drüsselchind plebanus in Moltzpüchel 1415 I 10. MUW 1, 1406 I R 2? (Erasm. Drusselkind de Salina)

- de Valle Eni 1406 II 9. MUW 1, 1403 I A 2

- Freyberger canonicus ecclesie Salczburgensis 1422 II 6. MUW 1, 1422 II R 13 (Erasm. Freyberger, can. Salczpurgensis)

- de Lantzhuta 1417 I 4. MUW 1, 1407 II R 20? (Erasm. Snablinger de Lanshůt); Uiblein, AFA I, 510

(Erasm. de Lanczhuta)

- de Reichersperg canonicus regularis $1437 \mathrm{I} 1$; bacc. in decr. $1440 \mathrm{I}$ 7. MUW 1, 1437 I R 74 (Erasm. Czachreis de Reichensperg)

- Reyntaler 1408 I 7. MUW 1, 1408 I R 30 (Erasm. Reyntaler, plebanus in Haynburga)

- Rubennaw de Gribswaldis 1418 II 20. MUW 1, 1416 I S 2 (Erasm. Rubenow)

- Teisser plebanus in Virgen de Carinthia 1436 II 6. MUW 1, 1427 I A 34? (Erasm. Teyser de Prawenecka)

Erhardus

- de Asparn Mayori 1441 I 1 (als b. a.). MUW 1, 1430 I A 44? (Erhard. Haydel de Asparn)

- Kornmecz de Prugk 1440 I 3. MUW 1, 1434 I A 20 (Erhard. Kornmesser de Prucka)

- Fabri canonicus ecclesie Sancti Viti Heridensis 1430 II 23. MUW 1, 1431 I R 52? (Erhard. Fabri de Eystauia)

- Herrant (de Newmburga Claustrali) 1422 I 20 (als m. a.); bacc. in decr. 1425 II 3; lic. in decr. 1430 I 30; dr. decr. 1432 I 15; jur. Dekan 1430 II, 1433 II (als Passauer Offizial). MUW 1, 1416 II A 4 (Erh. Herrant de Nuwenburga)

- de Sancto Egido de Nürmberga 1437 II 12. MUW 1, 1437 II R 2 (Erh. de Nürmberga)

- Satelboger canonicus ecclesie Rati- 
sponensis 1414 II 5. MUW 1, 1412 I Erkengerus

R 37

- de Sawnsheim canonicus Herbipolen-

- de Wolfach 1437 I 13 (als m. a.);

bacc. in decr. 1441 II 7. MUW 1, sis 1418 I 10. MUW 1, 1418 I R 27

1423 I R 6 (Erasm. Heging de Wolfach)

Ernestus

- Mèssemperger plebanus in Grawscharn 1421 II 11. MUW 1, 1420 I

A 37 (Arnestus Mèssemberger)

Fabianus

- de Tardaschedy (Tardascheydn) 1420 I 11; bacc. in decr. 1421 I 18; lic. in decr. 1424 I 21; dr. decr. 1424 II 13. MUW?

Valentinus

- de Mendscenth 1433 I 10. MUW 1, 1433 II H 3 (Val. de Meudscenth)

- Miskowicz 1423 I 14. MUW?

- de Mulembach in Septemcastris 1437 II 11 (als b. a.). MUW 1, 1435 I H 1 (Val. de Mulembach)

Vincencius

- plebanus in Haydendarff 1430 II 10. MUW?

- de Jordansmúl canonicus Wratislauiensis 1418 I 4; bacc. in decr. 1420 I 19; lic. in decr. 1421 I 6. MUW 1, 1418 I H 10

- Waller 1416 I 9. MUW 1, 1408 II A [sic!] 19 (Vinc. de Odemburg); Uiblein, AFA I, 510 (Vinc. de Sopronio)

- Weinstok de Holenburg 1409 II 8. MUW?

Virgilius

- Albner de Salzburga 1420 II 20. MUW 1, 1418 II $^{1}$ R 9 (Virg. Almer Vitus de Salzburga)

- de monasterio Admontensi 1420 II 12. MUW 1, 1415 II A 26 (Vitus Prawn de Agmund OSB)

Florianus

- de Leibnitz 1430 I 12 (als m. a.). MUW?

- Im Wald de Sancto Floriano
1435 II 8. MUW 1, 1430 I A 15

(Florian., filius Thome camerarii domini N. prepositi de Sancto Floriano)

Franciscus

- Althaymer lector ordinis Minorum in Nordlinga 1423 II 8. MUW 1, 1423 II R 76 (Franc. Althaymer de Nordlinga ordinis fratrum minorum)

- Granatoris de O̊ttingen 1410 II 1. MUW 1, 1410 II R 22 (Franc. Granatoris)

- Johannis de Luko tavernicorum regalium magister regni Vngarie 1425 I 3. MUW 1, 1425 I S 2? (Franc. Johannis de Luko)

- Resil (Resel, Rossel) (de Prusia) 1414 I 20; bacc. in decr. 1416 I 32; lic. in decr. 1416 II 37; dr. decr. 1416 II 41. MUW 1, 1414 I S 17 (Franc. Resper de Prussia)

- Rewtter de Nórlingen 1439 II 1 (als lic. a.). MUW 1, 1435 I R 38 (Franc. Rewtter de Narlinga)

- (Woiczdorff de Legnitz) canonicus ecclesie collegiate Libnicensis 1429 I 2 (als m. a. und bacc. in theol.); bacc. in decr. 1430 I 29; lic. in decr. 1434 II 14; dr. decr. 1434 II 15. MUW 1, 1429 I H 16 (Franc. Waiczdorf de Legnitz, mag. in art., bacc. in theol., can. eccl. collegiate Legnicensis); Uiblein, AFTh 2, 640 (Franc. Voitsdorfer)

Franco

- de Cronemberg 1414 I 3. MUW 1, 1414 I R 22 
Fridericus (Fridricus, Fredericus, Ffredericus)

- plebanus in Albeins 1403 I 3. MUW 1, 1403 I A 7 (Frider. plebanus in Alwins)

- Kraft (Krafft) 1406 II 15; bacc. in decr.1417 II 6; lic. in decr. 1417 II 7; dr. decr. 1418 I 3; jur. Dekan, can. Patauiensis und plebanus in Falkenstein 1418 I. MUW 1, 1401 II A1 bzw. 1401 II R 42 (dort getilgt); Uiblein, AFA I, 511

- Flokch de Kornburg 1440 II 7 (als b. a.). MUW 1, 1437 II R 35 (Frider. Floch de Chorenburg)

- Grenn de Rotemberga 1416 II 9. MUW 1, 1416 II R 10 (Frider. Green de Rotenberga)

- Prenner 1414 I 27; bacc. in decr. 1414 I 28; lic. in decr. 1414 II 16. MUW 1, 1391 I R 12 (Frider. Prenner de Ratispona); Uiblein, AFA I, 512; Schrauf, AFM 1, 103 (Fridr. Brenner, licentiandus in fac. iur. canon.)

- Purger de Helpach 1419 I 20 (als m. a.). MUW?

- Reschel plebanus in Vahendorf bacc. in decr. 1423 I 32. MUW?
- de Salczpurga 1414 II 11. MUW 1, 1409 I R 61? (Frider. Hartwerger de Salczburga); Uiblein, AFA I, 512? (Frider. de Salczburga)

- Sartoris alias Maler rector ecclesie parrochialis in Pirchachhausen (Birchachhusen) 1429 II 6; lic. in decr. 1435 I 15; bacc. in decr. und dr. decr. 1435 II 14. MUW?

- Schenkch de Lymburg Herbipolensis, Bambergensis etc. ecclesiarum canonicus 1411 II 8. MUW 1, 1411 I R 95 (Fridr. Schenk, can. Herbipolensis, Babenwurgensis, Patauiensis ecclesiarum)

- Stawthaimer de O̊tinga 1407 II 3. MUW 1, 1407 II R 31 (Fridr. Stauthaymer de Otinga)

- Tifer de Constancia 1420 II 2. MUW 1, 1420 I R 74 (Fridr. Tyuer de Constancia)

- de Waldegk, canonicus et custos ecclesie Frisingensis 1416 I 2. MUW 1, 1415 I R 17? (Frider. de Waldekk, can. Maguntinensis)

- Warnhofer de Astauia 1413 I 8. MUW 1, 1410 II R 45? (Frider. Warhofer de Mo̊sprun)
Gabriel

- de Augusta 1427 I 2. MUW?

- Lang de Augusta 1435 I 6. MUW 1, 1429 I R 35

- canonicus regularis de Wratislauia 1442 I 1. MUW 1, 1442 I H 16

Gebso

- Heawler de Constancia 1426 I 2. MUW 1, 1420 I R 85 (Geàpso Herdler de Constancia)

Georgius (Georius)

- Ekcherl plebanus in Gurkchueld 1421 I 3. MUW 1, 1419 I A 8? (Georg. de Gurkueld)
- Albrechtzperger 1421 I 8. MUW 1, 1414 II R 45 (Georg. Albrechtsperger de Salczburg)

- canonicus ecclesie Baciensis 1440 I 6. MUW 1, 1439 II H 21 (Georg. de Czenthe, can. Baciensis)

- plebanus in Barenghůt in Septemcastris 1437 II 7. MUW 1, 1438 I H 6 (Gregorius [sic!], plebanus in Barakwt)

- de Battaw canonicus Patauiensis 1415 II 13. MUW 1, 1415 II R 66 (Georg. Rotawer, can. eccl. cathedralis) 
- cappelanus 1414 I 17 (vgl. Paulus de Sana). MUW 1, 1414 I H 2 (Geor. professus eiusdem monasterii et capellanus dom. abbatis)

- de Corona (de Septemcastris) 1433 II 18; bacc. in decr. 1436 II 16; lic. in decr. 1440 I 11 (pro repetitione ad licenciam). MUW 1, 1429 I H 31

(Gregor. [sic!] de Corona) oder 1430 I H 22 (Georg. Pistoris de Corona)

- de Egerzeg (Egersek, Vngarus), 1424 I 10; bacc. in decr.1427 II 14; lic. in decr. 1430 I 31?; dr. decr. 1430 II 36. MUW?

- de Varembach 1413 I 21. MUW 1, 1412 II R 16 (Geor. de Farenpach)

- Vischlein (Vischel) 1416 I 8; bacc. in decr. 1419 I 2. MUW 1, 1416 I R 6 (Vislyn)

- de Herczogburg (Herczogburga) 1435 II 1 (als m. a.); bacc. in decr. 1440 II 18. MUW 1, 1423 I A 33

- [Heimburg de Sweinfordia] frater eius (fälschlich für Gregorius; immatr. gemeinsam mit Bruder Johannes) 1419 I 19. MUW 1, 1413 II R 79 (Gregor. Heymburg de Sweynfurt); vgl. Uiblein, AFA I, 531 (siehe den Eintrag unter Johannes Hainburkch de Sweinfordia)

- Hohenloch de Bamberga 1435 I 1 (als m. a.). MUW 1, 1430 II R 4 (Geor. Hohenloch de Papenberg)

- Institoris de Lobyn (Lóben) 1419 II 9; bacc. in decr. 1421 II 17. MUW 1, 1419 II $^{2} 10$

- Jågenreàwtter (Jåggenreiter, Jegenrewtter) de Patauia 1428 II 7; bacc. in decr. 1431 I 12; lic. in decr. 1433 II 15 (als plebanus in Varichdarff). MUW 1, 1420 I R 83 (Georg. Jengrewtter de Patauia)

- Layminger 1406 II 7. MUW 1, 1406 I R1 (Geor. Laymingêr, can. Frisingensis)

- Liechtenberg canonicus ecclesie Beate
Marie Glogouie Maioris 1412 I 24. MUW 1, 1412 I H 42 (Georg. Lietenberg)

- Melchaymer de Prawnaw 1408 II 1. MUW 1, 1408 II R 1 (Geor. Melkchaymer)

- Murrlein plebanus in O̊́czing 1415 I 17. MUW?

- canonicus regularis Neunburgensis 1406 II 3. MUW 1, 1405 I A 4

(Geor. de Petronella)

- Petri de Briswicz tavernicorum regalium magistri regni Vngarie 1425 I 2. MUW 1, 1425 I H 2

- Plo̊ntinger 1429 II 7. MUW 1, 1429 II R 15? (Geor. Plinthaymer)

- de Poppenhaim marscalc[us] 1413 I 31. MUW 1, 1412 II R 35

(Geor. Marschalich, can. Astauiensis)

- de Rudlinga 1415 I 20. MUW?

- plebanus in Schatschelkch (Schadschek) Septemcastrensis 1430 II 19; bacc. in decr. 1433 II 12. MUW 1, 1431 I H 15 (Geor. de Schatschelk Septemcastrensis)

- de Schawmberg canonicus Bambergensis 1415 II 9. MUW?

- Schrekch de Schårding 1441 I 5 (als m. a.). MUW 1, 1434 II R 18 (Geor. Schrik de Scherdinga)

- prepositus ecclesie Sepusiensis 1412 I 21. MUW 1, 1409 II H 15 (Jeorius Petri de Palucz); Uiblein, AFA I, 514 (Georg. prepositus Czipsensis [Zipsensis]); Uiblein, Mittelalterliches Studium, 121

- Stẻninger canonicus ecclesie Beate Marie in Fewchtwang 1431 II 8. MUW 1, 1431 II R 26 (Geor. Steyniger, can. Faichtbacensis, studens juris)

- Swentenkrieg de Welss 1435 II 7. MUW?

- de Wacia canonicus ecclesie Waciensis 1431 II 7. MUW 1, 1431 II H 22 (Geor., can. eccl. Baciensis)

- Wispeck 1418 II 10 (als m. a.). MUW 1, 1388 I R 18 (Geor. Wis- 
pech); Uiblein, AFA I, 514 (Georg. Wispekch); Uiblein, Mittelalterliches Studium, 121

- de Zůmelsperg plebanus in Grifen 1414 I 9. MUW 1, 1408 II A 9 (Geor. de Czumelsberg); Uiblein, AFA I, 515 (Georg. de Zömelsberg); Uiblein, Mittelalterliches Studium, 122

Gerhardus

- Vischpekch 1405 Dezember 17; 1405 II 3 (inkorporiert als dr. decr. und m. a.); jur. Dekan $1411 \mathrm{I}$, 1415 I, 1417 II (als Kanoniker von St. Stephan in Wien). MUW 1, vor 1377 Juni 24, 8 (Mag. Gerhard. lector ordinarius Wiennensis); MUW 1, 1381, circa Juni 13 (Vizerektor und Rektor der Wr. St. Stephansschule); Rektor 1388 II; Uiblein, AFA I, 515; Uiblein, Mittelalterliches Studium, 122

Giselherus

- Dobberkow jur. Dekan 1401 II und 1403 I (als dr. decr.). MUW 1, 1384 II 59 (als bacc. in decr.); Rektor 1390 I (als lic. in decr.) und 1402 I (als dr. decr.); Uiblein, AFA I, 515

Gottfridus (Gotfridus)

- (Fryling) de Oyta 1412 II 1; bacc. in decr. 1415 I 13. MUW 1, 1406 I S 2; Uiblein, AFA I, 515 (Gotfr. Vriling de Oyta)

- Harscher decanus ecclesie Augustensis 1411 II 19. MUW 1, 1411 II R 9 (Götfr. Harscher, decan. eccl. Augustensis); Rektor 1412 I; Uiblein, AFA I, 515

Gotthardus (Gothardus)

- de Altach Inferiori 1413 II 10.
MUW 1, 1394 II R 23? (Gothard. professus OSB de Inferiori Altach, iurista)

Gregorius

- Andree de Molhausen 1442 I 7 (als m. a.). MUW 1, 1437 I S 2 (Gregor. Andree)

- de Cosula vicarius ecclesie Bratislauiensis 1416 I 12. MUW 1, 1416 I H 1 (Gregor. [de] Cosla)

- de Valle Sancti Georgii canonicus ecclesie Strigoniensis 1435 I 2 (als m. a.); bacc. in decr. 1437 II 16; lic. in decr. 1439 II 7; dr. decr. 1439 II 9. MUW 1, 1423 II H 31 (Gregor. de Ualle sancti Georii)

- Grunt de Strigonia 1416 I 22. MUW 1,1416 I H 21 (Gregor. Grůnt de Strigouia)

- de Landestrost 1414 II 15. MUW?

- Ledenter canonicus ecclesie Novi Monasterii Herbipolensis 1412 II 5. MUW 1, 1412 II R 5 (Gregor. Ledencher de Herbipoli)

- Molitoris de Noua Ciuitate 1429 II 16. MUW 1, 1426 I H 22 (Gregor. Molitoris de Noua Ciuitate Voitlant)

- de Monte Sancti Georii Vallis Eni 1413 II 3. MUW?

- Scharendorf de Stuckardia bacc. in decr. 1433 I 15 (als m. a.). MUW 1, 1418 I R 7 (Gregor. Kankchel de Schorndorff)

- Weytensdorffer 1434 II 7. MUW? Gundakerus

- Aspekch bacc. in decr. 1410 II 3. MUW 1, 1384 II 153 (Gregor. de Obernperg Patauiensis diocesis); Uiblein, AFA I, 516 (Gundakerus de Oberbach)
Hartungus

- de Cappell rezipiert als utriusque iuris dr. ordinarius 1432 I 7; jur.
Dekan 1433 I (als canonicus ecclesie Patauiensis). MUW 1, 1432 I R 1 (Hartungus de Cappel, utriusque 
juris dr., can. Patauiensis); Uiblein, AFTh 2, 647

Hartwicus (Hertwicus, Herdwicus)

- Hawnsperger 1410 I 16. MUW 1, 1409 II R 9 (Hertw. Hawnsperger de Salczburga)

- Lampotinger (Lampolting) 1431 I 1 (als m. a.); bacc. in decr. 1435 II 19; lic. in decr. 1438 I 16; dr. decr. 1438 I 18. MUW 1, 1417 II R 24 (Hartw. Lampottinger)

- de Zumelsperg plebanus in Griuen 1421 I 2. MUW 1, 1415 II A 15 (Hertbicus de Czumelsperga)

Henningus

- de Halberstat 1414 I 22. MUW 1, 1401 II S 11? (Henningus Derwedewen de Halberstatt)

- Lủbersdorp 1414 I 13. MUW 1, 1414 I S 14 (Henningus Lubberstorp)

Henricus (Heinricus, Hainricus, Heynricus, Hinricus)

- de Bamberga familiaris eiusdem (sc. Georius de Schawmberg) 1415 II 10. MUW 1, 1411 I R 59? (Heinr. Ydelman de Babenberga); Uiblein, AFA I, 518? (Henr. Edelman de Babenberga)

- (de) Bernsteyn (Bernstayn, Pernstayn) (als m. a.) 1402 I 2; dr. decr. 1406 Februar 4; jur. Dekan 1405 II. MUW 1, 1401 II S 10 (Mag. Heinr. Bernstein); Uiblein, AFA I, 517 (Henr. [de] Bernstein)

- Burggrauius canonicus ecclesie Augustensis 1423 I 9. MUW 1, 1422 I R 34 (Heinr. Burgrafii)

- de Campa decanus ecclesie Opoliensis 1430 I 22. MUW?

- Kitzpühel jur. Dekan 1407 II und 1410 I (als dr. decr.). MUW 1, 1408 I Rektor; Uiblein, AFA I, 517; Uiblein, Mittelalterliches Studium, 122; Uiblein, AFTh 2, 648

- Clodebogk (Klodebokch) 1420 I 12; bacc. in decr. 1423 II 11; lic. in decr.
1430 I 27. MUW 1, 1417 I H 1

(Heinr. Clodbog)

- Klodechk 1427 II 3. MUW 1, 1427 II H 2? (Heinr. Klodbog)

- de Choburg 1422 I 16. MUW 1, 1422 I R 56 (Heinr. de Choburkch)

- Copericz de Budesheim 1417 I 20 (als m. a.). MUW 1, 1413 II H 47 (Heinr. de Budessin); Uiblein, AFA I, 517f. (Henr. Coppericz)

- Kranpergher canonicus Ratisponensis 1403 I 1. MUW 1, 1403 I R 1 (Hainr. Chronnperger); Uiblein, AFA I, 518 (Henr. Cranperger)

- Deringaw 1413 I 10. MUW?

- Eglinger de Waibling 1417 I 14. MUW 1, 1417 I R 61 (Heinr. de Wablingen) oder MUW 1, 1417 I R 91 (Heinr. de Wảblingen)

- Engelfrid de Spira 1422 I 12. MUW 1, 1412 I R 110? (Henr. Enegelfridi de Spira)

- de Valle Eni 1406 II 10 (als m. a.). MUW 1, 1403 II A 12? (Heynr. de Valle Eni)

- Fewchter (Feuchter) 1419 II 3; bacc. in decr. 1430 II 27. MUW 1, 1419 I A 3 (Heinr. Faüchter de Perg)

- Fleckl prepositus ecclesie Ardacensis 1438 II 14. MUW?

- Gastmeister (Gastmeyster) de Gobin (Gobyn) 1415 I 2; bacc. in decr. 1417 I 8. MUW 1, 1415 I H 5

- Haappel 1426 I 3 (als m. a.). MUW 1, 1420 I R 6? (Heinr. Heppel de Grossertor Chiemensis diocesis)

- de Helmstat prepositus Spirensis 1413 I 15. MUW 1, 1399 II R 7 (Henr. de Helmstad can. Spyrensis); Uiblein, AFA I, 518 (Henr. [de Helmstat] prep. Spirensis) ; Uiblein, Mittelalterliches Studium, 122

- de Heawen cantor et canonicus Argentinensis 1421 II 9. MUW?

- Hülger de Eberspewnt 1431 II 16. MUW?

- de Inferiori Altach ordinis Sancti Be- 
nedicti 1412 II 8. MUW 1, 1412 II R 15

- de Lawffen 1408 II 3. MUW 1, 1398 II A 26? (Henr. de Lauffen)

- Megenhart de Blabüren 1424 I 4. MUW 1, 1421 II R 20? (Hainr. Medardi de Blaburen)

- de Múldorf 1407 II 4. MUW?

- de Napurga 1421 II 20. MUW 1, 1415 I R 56 (Hainr. Prapacher de Napurga)

- Nosticz 1421 I 25. MUW 1, 1420 II H 34

- de Oyta 1414 I 5. MUW 1, 1412 II S 2

- Pawr de Nürmberga 1441 I 3. MUW 1, 1441 I R 33

- Plesner de Eschenbach 1430 I 14 (als m. a.). MUW 1, 1425 II R 21 (Henr. Plessner de Eschenbach)

- Prepticz de Slesia 1427 I 6. MUW 1, 1424 II H 8? (Henr. Preticz de $\mathrm{K}^{\mathrm{e}}$ oben)

- Rawch (de Tagersheim) 1417 I 17; bacc. in decr. 1421 I 16. MUW 1, 1411 II R 55 (Heinr. Rauch de Tagershaym)

- Reynwalt 1437 I 7 (als b. a.). MUW?

- Roll plebanus in Carlowicz necnon canonicus Chremsirensis 1414 II 12. MUW 1, 1414 II R 35 (Henr. Roll plebanus in Carlowicz)

- Roraw 1427 I 7. MUW 1, 1427 II H 1

- Rosemberg canonicus ecclesie Waciensis 1416 II 28 (als m. a.). MUW 1, 1416 II H 38 (Henr. de Rosenberg mag. in art. et bacc. medicine, can. eccl. Waciensis kathedralis)

- Roter 1419 I 16. MUW 1, 1418 II $^{1}$ R 45 (Heinr. Schemel de Rot)

- plebanus in Scheybs (Scheibs); bacc. in decr. 1421 II 18; lic. in decr. 1429 II 27. MUW?

- Scriptoris de Frankfordia bacc. in decr. 1418 II 8. MUW 1, 1413 II R1

- Slick prepositus in Northusen 1432 I
11 (als m. a.). MUW 1, 1426 I H 18 (Hainr. Slichk, [can. in Prunn])

- Stawthaymer 1409 I 6. MUW?

- Stoll de Hamelburg 1414 I 15 (als m. a.). MUW 1, 1409 I R 6; Uiblein, AFA I, 520; Schrauf, AFM 1, 107 (Mag. Henr. Stoll de Hammelburg, ciuis Wiennensis, doctor med.); AFM 2, 258 (Mag. Heinr. Stoll, art. et med. doctor)

- Streytperger canonicus Ratisponensis 1422 I 6. MUW 1, 1422 I A 7? (Heinr. Streitperger can. eccl. Frisingensis)

- Tandorf(f)er (de Nuremberga) 1413 II 9 (als m. a.); bacc. in decr. 1417 II 1; lic. in decr. 1418 II 5; dr. decr. 1419 I 17. MUW 1, 1401 II R 24( Hainr. Dandorffer de Nưremberga); Uiblein, AFA I, 520 (Henr. Tandorffer); Uiblein, Mittelalterliches Studium, 122; Uiblein, AFTh 2, 650 (Henr. de Tandorff)

- Wichtorff 1403 I 4. MUW 1, 1403 I R 22 (Hainr. Wychdorff)

- Wismayr 1433 I 11. MUW 1, 1433 I R 26 (Heinr. Wismaier de Chnütel)

- Wlpis de Syndring 1414 I 14. MUW 1, 1414 I R 80 (Henr. Fuchs de Sindringen)

- Wollemberg de Wismaria 1418 II 18. MUW 1, 1417 I S 8 (Heinr. Woldewerch)

- de Wyla 1436 II 7 (als m. a.). MUW 1, 1429 I R 68 (Henr. Stainhåwl de Wyla)

Hermannus

- Burrser de Veltkilch 1409 I 3. MUW 1, 1403 I A 25; Uiblein, AFA I, 521; Uiblein, Mittelalterliches Studium, 122

- de Cassela 1416 II 3. MUW 1, 1416 I S 9 (Herm. Stocz de Cassil)

- Deys beneficiatus in ecclesia Paderburnensis 1417 II 14. MUW 1, 1417 II S 6 (Herm. Deys de Paderburnensi ciuitate) 
- episcopus Frisingensis 1416 I 1. MUW 1, 1416 I A 1

- Haidelshaim 1413 I 16 (als m. a.). MUW 1, 1413 I R 59 (Mag. Herm. Heylman de Heydelsheim); Uiblein, AFA I, 521 (Herm. Heylman de Heydelßhein); Uiblein, Mittelalterliches Studium, 123; Uiblein, AFTh 2, 650

- Huxer de Pruscia 1430 II 1. MUW 1, 1430 II S 2 (Herm. Hůxer de Gdansk)

- Wulco de Frankenfordis 1417 II
9 (als m. a.). MUW 1, 1417 I S 6 (Mag. Herm. Wolko de Franckenfordis)

Hildebrandus (Hilprandus)

- Oder de Dorffen 1429 II 10. MUW?

Hodiko

- plebanus in Radhost 1417 II 11. MUW 1, 1417 II H 26 (Hodyco pleHugo banus in Radhost)

- de Leynsteten abbas Alperspacensis 1420 II 11. MUW 1, 1420 II R 1
Jacobus

- Angeli de Vlma 1417 II 12 (als m.a.). MUW 1, 1412 I R 19; Uiblein, AFA I, 522; Uiblein, Mittelalterliches Studium, 123

- de Baden 1414 II 17 (als m. a.). MUW 1, 1395 I A 32 (Jacob. Vngeriht de Baden); Uiblein, AFA I, 522 (Iacob. de Baden); Schrauf, AFM 1, 103 (Mag. Iacob. de Baden, licenciandus in fac. iur. canon.)

- Bawdewek plebanus in Nissa 1416 II 26. MUW 1, 1416 II H 35 (Jacob. Pandeweck plebanus in Nyssa)

- Blassemberger de Perawit 1419 I 15. MUW 1, 1414 II R 31 (Jacob. Plassenberger)

- de Chweffstain 1426 II 9; bacc. in decr. 1426 II 22. MUW?

- de Chlausenburga (Clausenburk) 1428 I 8; bacc. in decr. 1430 II 29. MUW 1, 1413 II H 8 (Jacob. de Clausemburg)

- Ebser lic. in decr. 1439 I 11; dr. decr. 1439 I 13. MUW 1, 1422 I R 11? (Jacob. Ebser de Salczpurga)

- Frieshaymer de Schirling 1422 II 13 (als m. a.). MUW 1, 1414 II R 50? (Jacob. Frieshaimer de Ratispona)

- de Gilgenburg 1412 I 9. MUW?;
Uiblein, AFA I, 523; Uiblein, Mittelalterliches Studium, 123

- Hofeman de Onalbach 1405 II 1. MUW 1, 1405 II R 1 (Jacob. Hofman de Anolspach)

- de Inferiori Stokchstal 1416 I 15 (als m. a.). MUW 1, 1407 I A 34 (Jacob. Vectoris de Niderstokchstal); Uiblein, AFA I, 523 (Iacob. de Stockstal); Uiblein, Mittelalterliches Studium, 123; Schrauf, AFM 1, 107 (Mag. Iacob. de Stokchstall [Stokstal, Stochkstall], doctor med.); Schrauf, AFM 2, 258 (Mag. Jacob. [de] Stokkstal, [Stokchstal, Stokstal], doctor med.)

- Johannis de Mưsson 1422 I 5. MUW 1, 1421 I R 55

- presbyter de Lanczans 1409 I 7. MUW 1, 1409 I H 10 (Jacob. de Lanczaus)

- Luf de Weyssemburga famulus 1422 II 7. MUW 1, 1422 II R 7

- O̊ch de Pappenhaim 1424 I 7. MUW 1, 1421 I R 26 (Jacob. Ach de Pappenhaim)

- Pistoris de Nissa 1416 I 5. MUW 1, 1415 II H 35

- Rayner plebanus in Cirkla 1434 I 2. MUW 1, 1433 II A 11

- de Rosenaw 1430 I 9. MUW 1, 1429 II H 30 (Jacob. de Rosenauia) 
- Rosenczwey de Weyssemburga lic. in decr. 1442 I 11. MUW?

- Rosenczweig de Newburga 1436 II 5 (als b. a.). MUW?

- de Sancto Vito plebanus in Gneàs 1414 I 23. MUW?

- de Sancto Zenone 1412 I 1. MUW 1, 1411 I R 74 (Jacob. de Richenhall can. eccl. Senonis)

- de Strigonio bacc. in decr. 1432 II 10 (als m. a.); lic. in decr. 1434 II 8; dr. decr. 1434 II 12. MUW 1, 1422 II H 21 (Jacob. Nycolai de Strigonio)

- Tols de Rudlinga 1429 II 2 (als m. a.). MUW 1, 1422 II R 70 (Jacob. Tulcz de Rotlinga); Schrauf, AFM 1, 107 (Mag. Iacob. Tolcz de Ro̊dlinga, doctor med.)

- de Weytra 1413 I 20. MUW?

Jaroslaus (Jeroslaus)

- de Sluskow canonicus Cracouiensis (als m. a.) 1426 II 1. MUW 1, 1426 I H 68 (Jaruslaus de Slußew mag. in art. et can. eccl. Cracouiensis necnon rector parrochialis in Gnoyno)

Jeronimus

- Beckensloer de Wratislauia 1439 I 5. MUW 1, 1438 II H 42 (Jeron. Pekchenslaher de Wratislauia)

- Vogelsang (Vogelsangk) canonicus Olomucensis 1437 I 19 (als m. a.); bacc. in decr. 1439 II 3. MUW 1, 1429 I H 26 (Jeron. Vogelsankch de Olomuncz); Uiblein, AFTh 2, 652 (Ieron. [Vogelsang] de Olmucz)

- (Lantuoyt) de Olmüncz 1428 I 6 (als m. a.); bacc. in decr. 1430 I 33; lic. in decr. 1433 II 14. MUW 1, 1416 II H4 (Jeron. Venczlai de Olmucz)

- de Olmuncz 1429 II 8 (als m. a.). MUW?

- de Påmburg 1416 II 18. MUW 1, 1413 I R 77 (Jeron. de Pawmburg)

- Posser (de Salczburga) 1436 II 3 (als m. a.); bacc. in decr. 1439 II 4 (als plebanus in Hausdorf); lic. in decr. 1442 I 10. MUW?
Jodocus (Judocus)

- Gesler canonicus Eystetensis 1421 II 5. MUW 1, 1421 I R 3 (Jodoc. Gessler de Vlma)

- Hawsner (de Nouoforo) 1437 I 14 (als m. a.); bacc. in decr. 1440 I 10. MUW 1, 1425 II R 44; Uiblein, AFTh 2, 653f. (Iodoc. Hausner de Novo Foro cancellarius); Schrauf, AFM 2, 251 (Mag. Jodocus Hausner, doctor decretorum, officialis Patav[iensis])

- Me̊ntelberger de Ynsprucka 1422 I 15. MUW 1, 1419 I A 40 (Jodoc. Mentelberger de Inspruk)

- Maursteter de Campidona famulus 1438 II 9. MUW 1, 1438 II R 49 (Jodoc. Mawrstetter de Campidona)

- Quedenow de Künigsperg 1416 I 19. MUW 1, 1416 I S 4 (Jodoc. Quedenawer)

- Rot 1408 I 5. MUW?

- Rot de O̊berlinga (Vberling) 1420 I 5 (als m. a.); bacc. in decr. 1431 II 20. MUW 1, 1414 I R 101 (Jodoc. Rot de Vberlinga)

- Seler (de Esslinga) 1437 II 13 (als lic.in a.); bacc. in decr. 1440 II 17. MUW 1, 1432 I R 64 (Jodoc. Sayler de Eßlinga)

Johannes

- Årkenstainer prepositus Gurnocensis 1424 I 5. MUW 1, 1424 I A 10 (Joh. Ergkenstainer)

- Aichelperger (Aychelperger) de Nouoforo 1429 II 5; bacc. in decr. 1432 I 18; lic. in decr. 1435 I 10. MUW 1, 1425 II R 9; Schrauf, AFM 2, 247 (Mag. Ioh. Aichlperger de Novoforo, officialis curiae Patav[iensis])

- de Alba Jula (Albagywla) 1437 I 4; bacc. in decr. 1438 II 22. MUW 1, 1431 II H 8 (Joh. Anthonii de Albaghüla)

- Albrand de Bruchsella 1412 I 13. MUW 1, 1412 I R 86 (Joh. Albrant de Brichsella) 
- de Altamuta 1432 I 10. MUW 1, 1426 I H 39

- de Altemburg 1411 I 17. MUW 1, 1409 II S 2 (Joh. Altenwrg de Insula?)

- Ambrosii (de Gvnczpurg) 1423 I 19 (als m. a.); bacc. in decr. 1426 I 11. MUW 1, 1423 I A 43 (Mag. Joh. Ambrosii de Gưnczpurg)

- Amelprecht de Nicolspurga 1417 II 10. MUW 1, 1416 II H 46

- Amselèr 1410 I 12. MUW 1, 1410 I A 31 (Joh. Ampseelr de Curia)

- Arnolt de Tullaco 1429 II 11. MUW 1, 1425 I A 30 (Joh. Arnold de Toblaco)

- Augustini de Ratispona 1414 II 13. MUW?

- Awer canonicus Ratisponenesis 1416 I 24. MUW 1, 1416 I R 34

- Bachenstain (Pachenstain) bacc. in decr. 1430 I 28 (als m. a.); lic. in decr. 1431 II 18 (als archidiac. eccl. Zagrabiensis); dr. decr. 1432 I 16 (als can. Zagrabiensis). MUW 1, $1418 \mathrm{II}^{1}$ R 51 (Joh. Pachenstain de Hallis)

- Berwardi (als Rektor, m. a. und bacc. theol.) 1401 II. MUW 1, 1385 II A 27 und 1385 II R 27; zweites Rektorat 1403 I; Uiblein, AFA I, 525 (Ioh. Berwart de Vilingen); Uiblein, AFTh 2, 656 (Ioh. Berwart)

- de Bybraco 1418 II 3. MUW?

- de Bozna (Bossna) canonicus Casmensis bacc. in decr. 1428 II 11; lic. in decr. 1431 II 19 (als can. eccl. Zagrabiensis); vielleicht identisch mit Johannes de Zagrabia? MUW?

- Briger canonicus ecclesie Wratislauiensis 1433 I 2. MUW 1, 1433 II H 6

- Brisach de Constancia 1415 I 19. MUW 1, 1415 I R 31

- de Brixna bacc. in decr. 1427 I 11. MUW?

- Brucenus bacc. in decr. 1415 II 5. MUW 1, 1409 I H 4 (Joh. Pruteni de Barka?)
- de Bruchenstain 1427 I 3 (als m. a.). MUW?

- Butczpach 1410 I 17 (als m. a.). MUW 1, 1401 I R 20 (Joh. de Buczbach) oder 1402 II R 16? (Joh. Swab de Buczpach); Uiblein, AFA I? (Joh. Swab [de] Butzbach); Uiblein, Mittelalterliches Studium, 125?

- Kèser commendator domus Sancti Johannis apud Sanctum Petrum in Kar[inthia] 1440 II 3. MUW 1, 1435 II H 9? (Joh. Keser de Wratislauia)

- Kern de Kulsheim 1435 II 6 (als b. a.). MUW 1, 1430 I R 27 (Joh. Chern de Chůlshaim)

- de Kyczing 1422 I 17. MUW 1, 1422 I R 42 (Joh. Florem de Kiczingen)

- Kirchen iunior 1414 II 7 (als m. a.). MUW 1, 1414 II R 7 (Joh. Chirichhaym de Heidelberg)

- Chlosner canonicus Patauiensis 1419 I 7. MUW 1, 1414 I R 96 (Joh. Chlawsner de Gern, can. Patauiensis)

- de Chochaim 1434 I 3. MUW 1, 1423 I R 55 (Joh. Cocheim)

- de Corona 1414 I 8 (als m. a.). MUW 1, 1409 I H 8? (Joh. de Corona) oder $1410 \mathrm{I} \mathrm{H}$ 15? (Joh. de Corona); Uiblein, AFA I, 530? (Joh. Goldner [Göldner] [de] Corona)

- de Corona (Rảbel) 1424 II 7; bacc. in decr. 1427 II 13. MUW 1, 1420 II H 20? (Joh. de Corona) oder 1422 II H 7? (Joh. Sutoris de Corona) oder 14124 I H 10? (Joh. Karig de Corona)

- Koslick de Podatzschin 1430 I 1. MUW 1, 1425 II H 46 (Joh. Chosslig de Podaczin)

- Cristoferi de Winczig 1413 II 15. MUW 1, 1413 II H 49

- de Chrussen (Crossna) (Toliatoris) 1429 I 3; bacc. in decr. 1430 II 26. MUW 1, 1425 II H 47? (Joh. Chrussen de Wratislauia) oder 1423 I H 21? 
(Joh. Plaw de Crusna) oder 1415 I H 56? (Joh. Lebgern de Crossen)

- Deys 1418 I 20. MUW 1, 1417 II S 7? (Joh. Deys de Paderburnensi ciuitate) oder 1418 I R 38? (Joh. Deys de Ratstat)

- de Dyessenhofen 1406 II 5. MUW? Uiblein, AFA I, 527 (Ioh. de Dýssenhoven); Uiblein, Mittelalterliches Studium, 123

- Doleatoris 1423 I 22. MUW 1, 1423 I R 141 (Joh. Doleatoris de Eystauia)

- de Ebersdorff 1426 II 16. MUW?

- Ebner de Chremsa 1420 I 8. MUW 1, 1414 II A 6 (Joh. Ebner de Chremsa)

- Ehinger de Landshůta 1441 II 4. MUW 1, 1438 I R 79

- de Eyb canonicus ecclesie Eystetensis 1423 I 1. MUW?

- de Eych 1423 I 20; jur. Dekan 1435 I (als dr. utr. jur.), 1437 II. MUW 1, 1423 I R 153 (Joh. Eych); Uiblein, AFTh 2, 659 (Ioh. de Eych); Schrauf, AFM 2, 247 (Ioh. Aich, jur. can. doctor, postea episc. Eystetensis)

- Elrichshusen 1423 II 3. MUW 1, 1408 II R 11? (Joh. Ellrichshawser)

- Erbeiter canonicus ecclesie Sancti Johannis in Hawg 1416 II 31. MUW 1, 1408 II R 22? (Joh. Arbaitter de Aw)

- Vey de Wratislauia, altarista ibidem 1421 I 24. MUW 1, 1420 II H 43? (Joh. Nicolai Fen de Wratislauia)

- Felber de Hallis 1413 I 9. MUW?

- Velbêr 1410 I 4. MUW 1, 1408 II A 15? (Joh. Velber de Wyenna)

- de Veneciis 1402 I jur. Dekan (als dr. decr.). MUW 1, 1400 II A 1 (Joh. de Garzonibus de Veneciis, m. a. et dr. iur. canonici, lector ordinarius in facultate iuris); Uiblein, AFA I, 529 (Ioh. de Garsonibus [Garzionibus]); Uiblein, Mittelalterliches Studium, 124
- Vetter de Memmingen 1414 I 12. MUW?

- Fyol de Toran 1418 I 15. MUW 1, 1418 I S 1 (Joh. Viol de Toren)

- Fleckel canonicus Wratislauiensis 1438 II 15. MUW?

- de Voburg 1410 I 15 (als m. a.). MUW 1, 1409 I R 31 (Joh. Ludwici de Voburg); Uiblein, AFA I, 529; Uiblein, Mittelalterliches Studium, 124

- Folczian de Wienna 1414 II 1. MUW 1, 1408 I A 12; Uiblein, AFA I, 529 (Joh. Volczian de Wyenna)

- de Frankfordia (Franckfordia) (Prunger) 1417 II 8 (als m. a. und bacc. in decr.); 1418 I 2 pro admissione ad repetitionem facultatis; lic. in decr. 1419 II 17; dr. decr. 1419 II 16. MUW 1, 1399 II R 1 (Joh. Brunger de Frankchfordia); Uiblein, AFA I, 529 (Joh. de Frankenfurt [Frankfordia]); Schrauf, AFM 2, 249 (Ioh. de Frankfordia, jur. canon. doctor, [Ioh. Renensis, Rinensis])

- Fuchs de Danczk 1425 I 4. MUW 1, 1418 II $^{1}$ S 2? (Joh. de Danczk)

- Fülstein de Bladen prepositus ecclesie Sancte Crucis Wratislauiensis 1436 II 1; bacc. in decr. 1441 II 6. MUW 1, 1436 II H 9 (Joh. Füllenstein)

- Füssel (Feuszel, Füssil) de Wratislauia (Bratislauia) 1411 II 9; bacc. in decr. 1416 I 28; lic. in decr. 1420 I 16; dr. decr. 1420 II 24. MUW 1, 1411 II H 22

- Gartner de Perching 1419 I 14 (als m. a.). MUW 1, 1407 I R 1? (Joh. Indemchelr de Perching); Uiblein, AFA I, 529 (Joh. Gartnar de Perching)

- Gawen de Lenicz 1412 I 16. MUW 1, 1412 I H 32 (Joh. Gaben de Jegenicz)

- Gebhardi plebanus in Enczestorf Maiori 1415 II 2. MUW 1, 1413 I A 45 (Joh. Gebhardi de Enczestorf) 
- Geltprecht (Gelprecht) de Lintz (Lynntz) 1438 II 1; bacc. in decr. 1440 II 16. MUW 1, 1435 I A 23

- de Gemundia 1427 II 1. MUW 1, 1417 I R 39? (Joh. Månndel de Gmünd) oder 1418 II $^{1}$ A 4? (Joh. Frizzichnicht de Gmunden)

- Gerhardi de Eysenhusen (Ysenhusen) 1415 I 9; bacc. in decr. 1424 II 15. MUW 1, 1411 R 105

- Giesser de Monaco 1440 II 6 (als b. a.). MUW 1, 1437 I R 28

- Glookelherr 1408 I 2. MUW 1, 1404 I R 17? (Joh. Glokkelherr de Kawffpuhel); Uiblein, AFA I, 530? (Ioh. Glógelher de Augusta)

- de Gmvnden 1421 II 13. MUW? Schrauf, AFM 2, 250? (Mag. Joh. de Gmunden, vicecancellarius)

- Gnechowicz de Wratislauia 1411 I 2. MUW 1, 1411 I H 2

- Gnendorffer custos et canonicus Ratisponensis 1422 I 13. MUW 1, 1422 I R 46 (Joh. Gnendorffer, can. Ratisbonensis)

- Godke de Wratislauia 1418 I 14. MUW 1, 1418 I H 3 (Joh. Gatko)

- Gödke de Danczk 1412 I 4. MUW?

- (Streit) de Goltperg presbyter Wratislauiensis diocesis 1435 II 9; bacc. in decr. 1437 II 20. MUW? Schrauf, NH, 433 (Mag. Joh. de Goldberg, Prok. der ung. Nation 1458 II, 1459 II, 1460 I, 1467 II, 1468 I; Proc. substit. 1461 I)

- de Gotwico 1442 I 2. MUW?

- Gotz de Studkardia 1429 II 24. MUW 1, 1427 II R 9 (Joh. Götz de Stůgardia)

- Graw 1432 II 2 (als m. a.). MUW 1, 1422 II R 76? (Joh. Graw de Gundelfing)

- [de] Grecz Regine decanus ecclesie collegiate Kremsirensis ac canonicus ecclesie Bratislauiensis 1427 II 4. MUW 1, 1427 II H 32

- Grüen de Hallis 1441 I 9. MUW 1,
1437 I R 78 (Joh. Gruelin de Hallis Sweuie)

- Grůenbald canonicus ecclesie Frisingensis 1411 II 13. MUW 1, 1411 II R 7 (Joh. Gruenwalder, can. maioris eccl. Frisingensis); Schrauf, AFM 2, 249 ([Ioh. Grüenwalder] card. et episc. Frising.)

- de Gürtesch 1436 I 4. MUW 1, 1423 I H 22? (Joh. de Kürtes)

- Gůt de Corona 1437 II 8 (als m. a.); bacc. in decr. 1440 I 8. MUW 1, 1410 I H 15? (Joh. de Corona) oder 1411 I H 12? (Joh. Lapicide de Corona); Uiblein, AFA I, 531 (Ioh. Güt de Corona)

- Gwerleich (Gwårleich) 1406 II 17; lic. in decr. 1419 II 21; dr. decr. 1421 I 5; jur. Dekan 1421 I (als dr. decr.), 1426 I, 1430 I, 1432 I (als m. a.), 1435 II. MUW?

- Haegg de Kirchain 1424 I 3. MUW 1, 1421 II R 19 (Joh. Hak de Kirchaym)

- Hewndel famulus (sc. Albertus de Potendorff) 1419 II 13. MUW?

- de Hallstat 1433 I 6 (als m. a.). MUW 1, 1425 I A 5? (Joh. Wolfingi de Halstadt)

- Harster de Lantzhuta familiaris eius (sc. Marcus Enenkel de Albrechtzperg) 1416 I 18. MUW 1, 1412 II R 24 (Joh. de Lannczhueta)

- Heller de Monaco 1437 I 15 (als m. a.). MUW 1, 1428 II R 28

- Heresinger de Strawbinga 1422 II 8 (als m. a.). MUW 1, 1411 I R 17? (Joh. Crismer de Strawbinga) oder 1411 II R 42? (Joh. Neunhauser de Straubinga); Uiblein, AFA I, 539? (Joh. de Straubinga)

- de Hermansdorf canonicus ecclesie Warmiensis 1419 II 11. MUW 1, 1419 II $^{2}$ S 10 (Joh. de Hermannstorff)

- de Herremberg 1414 II 6. MUW 1, 1409 II R 30 (Joh. Ehynger de Her- 
renberga); Uiblein, AFA I, 531? (Ioh. de Herrenberg)

- Hers (Hễz, Has) (de Brunna) canonicus ecclesiarum Brunnensis et Olomuncensis 1429 I 1 (als b. a.); bacc. in decr. 1432 I 20; lic. in decr. 1438 I 14. MUW?

- Hertfelt de Elwangen 1437 II 1. MUW 1, 1432 II R 19 (Joh. Hertfeld)

- Hess de Ysenach 1412 I 23; bacc. in decr. 1423 I 26; lic. in decr. 1423 I 27; jur. Dekan 1423 II. MUW 1, 1412 I S 11 (Joh. Hess de Eysnach); Uiblein, AFA I, 531 (Ioh. Hesse [Hess] de Ysenach]); Uiblein, Mittelalterliches Studium, 124

- Hieler de Ala 1429 II 23 (als lic. a.). MUW 1, 1426 I R 24 (Joh. Hiller de Aula)

- Hymel de Weytz 1411 I 8 (als m. a.). MUW 1, 1406 I A 3; Uiblein, AFA I, 532 (Joh. Himel de Weycz); Uiblein, Mittelalterliches Studium, 124; Uiblein, AFTh 2, 662

- Hindernpach 1439 I 10 (als m. a.). MUW 1, 1434 II R 4

- Hoffkircher de Wienna 1426 I 4. MUW 1, 1418 I A 10 (Joh. Hofchiricher)

- Hofleich (de Vlma) 1427 II 9 (als b. a.); bacc. in decr. 1432 I 19. MUW 1, 1424 I R 75

- Hofrichter de Hallis 1424 I 12. MUW 1, 1424 I R 86

- Holczman de Aw 1416 II 23 (als m. a.). MUW?

- Husel 1411 II 4. MUW 1, 1403 I A 36? (Joh. Husli de Altkilch)

- Yencz de Wratislauia 1419 I 4. MUW?

- Jeger 1429 I 16. MUW 1, 1429 I R 70 (Joh. Jèger de Vlma)

- professus monasterii Jerucensis Ordinis Premonstratensis 1417 I 12; bacc. in decr. 1422 I 18? (als can. Jerucensis). MUW?
- Jewkewicz de Possilwicz 1422 II 5. MUW 1, 1422 I H 39 (Joh. Jenekewicz de Posselbicz)

- Jonsdorf clericus Bratislauiensis diocesis 1412 I 25. MUW 1, 1412 I H 43

- Lamberg de Wratislauia 1420 II 4. MUW 1, 1419 I H 8 (Joh. Lemberg)

- Lapicide de Kunigshofen 1437 II 5 (als m. a.). MUW 1, 1428 I R 8 (Joh. Lapicida de Kungßhofen im Gew)

- de Lapide plebanus in Folxhaim 1415 I 16. MUW 1, 1415 I R 126 (Joh. de Lapide, pastor in Fohohaim)

- Lehener de Purchhawsen 1423 I 11 (als m. a.). MUW?

- de Leonrod canonicus ecclesie Eystetensis 1423 I 17. MUW 1, 1423 I R 138 (Joh. de Leonrad)

- Lewbner de Nova Civitate 1420 I 6. MUW 1, 1420 I H 16

- Loman de Riga famulus 1418 I 13. MUW 1, 1418 I S 4

- Ludberti canonicus Boldmensis 1415 II 8. MUW 1, 1415 II S 6 (Joh. Lodwici)

- Màgerl 1409 II 3 (als m. a.). MUW 1, 1402 II H 9? (Joh. Megerlein); Uiblein, AFA I, 533 (Joh. Magerl); Uiblein, Mittelalterliches Studium, 125; Schrauf, AFM 1, 105 (Mag. Ioh. Mẻgerl, doctor med.)

- de Manderscheyt canonicus ecclesie Treuerensis 1417 I 1. MUW 1, 1417 I R 22

- de Marichpurck 1410 I 3. MUW 1, 1403 I R 15? (Joh. Martorff de Marpurga) oder 1404 I R 8? (Joh. Bruning de Marpurg) oder 1409 I R 5? (Joh. Trappenstain de Marpurg); Uiblein, AFA I, 533? (Ioh. [de] Marpurk de Hassia); Uiblein, Mittelalterliches Studium, 125

- Maulperger de Altenmuldarff 1430 II 4. MUW 1, 1430 I R 103

- de Mawttarn 1420 II 15. MUW 1, 1415 I A 15 (Joh. de Mawtarn) 
- de Meyrs (Måyrzz) 1418 I 5; bacc. in decr. 1420 II 27; lic. in decr. 1422 II 10. MUW? Uiblein, AFA I, 533 (Ioh. de Mawrs [Meyrs]) ; Uiblein, Mittelalterliches Studium, 125

- Merswein de Argentina bacc. in decr. 1417 II 4 (als m. a.); lic. in decr. 1418 II 4. MUW 1, 1403 I R 60 (Joh. Merschwin); Uiblein, AFA I, 534

- Mùyther 1411 II 6. MUW?

- Münheym de Praga 1428 I 1. MUW 1, 1423 II H 10 (Joh. Munhein)

- Mulhaimer plebanus in Aussernczelln 1434 I 6. MUW 1, 1426 II R 9? (Joh. Streycher de Mùlheim)

- archidyaconus Newgradiensis in ecclesia Strigoniensi ac canonicus ecclesie Transiluanensis 1427 II 2. MUW?

- Newsteter canonicus in Anspach 1416 II 27. MUW 1, 1415 II R 4

- Nicolai de Brunna 1412 II 7. MUW 1, 1412 II H 17

- Nicolai Fabri de Olsna 1429 II 19. MUW 1, 1429 II H 35

- de Nydden 1410 II 5. MUW 1, 1410 I R 45 (Joh. de Niden)

- de Olderspach 1402 I 4 (als m. a.). MUW?

- de Olmuncz 1416 II 5. MUW 1, 1416 I H 31 (Joh. Cliuis de Olmůncz)

- Ortlini de Noorlinga 1440 II 1 (als m. a.). MUW 1, 1435 I R 57 (Joh. Ortel de Nårdlinga)

- Pêrtl (Bêrtel) de Salczbach (Sulczpach) 1438 II 5; bacc. in decr. 1441 I 11. MUW 1, 1433 II R 19 (Joh. Pèrtl de Sulczpach)

- de Palborn bacc. in decr. 1423 II 10 (als m. a.). MUW?

- de Pankotha 1441 I 10 (als m. a.). MUW 1, 1425 II H 40 (Joh. Augustini de Pankota)

- Parrewtter 1433 II 4. MUW 1, 1434 I R 23 (Joh. Berăutter de Rauchenkolben)
- Pawswågl de Olomutz 1441 II 3. MUW 1, 1437 I A 34 (Joh. Pawzwang de Olomuncz)

- Pencznalber Frisingensis et Augustensis ecclesiarum canonicus 1438 II 8. MUW 1, 1438 II R 46 (Joh. Pintznawer, can. Frisingensis)

- de Perchtoltzdorff canonicus regularis Neunburgensis (Newmburgensis) 1406 II 2; rezipiert als dr. decr. 1421 II 1; jur. Dekan 1422 I. MUW 1, 1405 I A 3 (Joh. de Berchtoldstorf); Uiblein, AFTh 2, 666 (Joh. de Perichtoltzdorff)

- Pflancz 1423 I 21. MUW 1, 1423 I R 140

- Pleyntinger 1417 I 11. MUW 1, 1413 II R 58? (Joh. Plătinger de Patauia)

- Pluemenekcher (Ploumenegker) 1434 I 14 (als b. a.); bacc. in decr. 1436 II 15; lic. in decr. 1439 II 10. MUW?

- Polzmacher (Poltzmacher, Polczmacher) (de Brunna, Brünna) 1428 I 4; bacc. in decr. $1430 \mathrm{I} 34$; lic. in decr. 1432 II 8; dr. decr. 1434 II 13; jur. Dekan 1436 I, 1439 I, 1441 I. MUW 1, 1422 I H 19 (Joh. Polczmacher de Brunna); Rektor 1438 I; Schrauf, NH, 405 (Dr. [Joh.] Polczmacher, prep. Brünnensis)

- Poter Sartoris de Fridwerkch 1429 I 15. MUW 1, 1422 II R 25 (Joh. Sartoris de Freytberg)

- Poueda 1416 I 10. MUW 1, 1416 I H 4 (Joh. de Pobedna)

- Preylant de Nissa 1416 I 6. MUW 1, 1415 II H 36 (Joh. Preytlant de Nysa)

- Preym de Cliuis 1416 I 7. MUW?

- Preller plebanus in Trafess 1423 II 2 (als m. a.). MUW 1, 1417 I R 36? (Joh. Prellăr de Ottinga)

- de Pudlino canonicus ecclesie collegiate Sancti Martini 1427 I 1; bacc. in decr. 1442 I 13. MUW 1, 1427 I 
H 27 (Joh. de Podolino, can. eccl. s. Martini in Scepücz)

- Pusmer de Nurmberga 1430 I 15. MUW 1, 1424 II R 25 (Joh. Pưßner)

- Quentin de Ortenberg 1433 I 4. MUW?

- Raczenhawsen plebanus in Wülfesheim 1421 I 14 (als m. a.). MUW?

- Rademan (de Lukau) altarista ecclesie Wratislauiensis 1430 I 5; bacc. in decr. 1434 I 18. MUW 1, 1430 I H 14 (Joh. Radman)

- Rătelchouer 1430 II 17 (als m. a.). MUW 1, 1423 I R 143 (Joh. Retelkofer de Purghausen)

- Rẽwtemberger plebanus in Tytmaning 1420 II 1 (als m. a.). MUW 1 , 1409 I A 50 (Joh. Rütenberger de Obernnassenfüs de Carinthia); Uiblein, AFA I, 536 (Joh. Reutenberger [Reutenperger])

- Raptoris 1432 I 13. MUW?

- custos Rathibornensis, bacc. in decr. 1438 II 11. MUW 1, 1438 I H 70

- de Redisch 1431 II 14 (als b. a.). MUW 1, 1427 II H 25

- Redler famulus eius (sc. Johannes Zink) 1413 II 14. MUW 1, 1412 I A 13 (Joh. Raedler)

- Reysegk de Reysspach 1434 II 4. MUW?

- Resch (de Swabach) 1428 II 1 (als b. a.); bacc. in decr. 1433 II 10. MUW 1, 1424 I R 100 (Joh. Rekch de Swabach)

- Rex 1409 II 6. MUW 1, 1409 II S 4 (Joh. Rex de Prussia)

- de Ringenstain 1442 I 6. MUW 1, 1438 I R 129 (Joh. Ringlstain de Esslinga)

- Ro̊chel 1408 I 1 (als m. a.). MUW 1, 1403 I R 61 (Joh. Ròchli de Argentina); Uiblein, AFA I, 536 (Joh. Rochel); Uiblein, AFTh 2, 668 (Ioh. Röchel)

- Ro̊tlini de Opauia 1428 I 5; vielleicht identisch mit Joh. Sews (siehe dort).
MUW 1, 1427 I H 24? (Joh. Sews de Oppauia)

- de Rosenaw plebanus ad Sanctum Johannem prope Grauenwerd 1421 I 11. MUW 1, 1411 I A 11 (Joh. de Rosnaw); Uiblein, AFA I, 537; Uiblein, Mittelalterliches Studium, 125

- (de Rotemberg) ordinis Premonstratensis 1423 I 10; bacc. in decr. 1426 I 12 (als can. reg. Ordinis Premonstratensis monasterii Roggenburgensis). MUW 1, 1423 I R 107 (Joh. Molitoris de Rokenburg, prof. O Prem.)

- (Rúel) de Francfordia (Franckfordia, Frankfordia) 1435 I 3; bacc. in decr. 1438 I 13; lic. in decr. 1441 II 5. MUW 1, 1427 II R 1

- Ruschlini alias Judicis de Gewicz 1416 I 16. MUW 1, 1409 II H 14 (Joh. Nicolai de Gewicz)

- Schachner professus monasterii Sancti Lamperti 1441 I 8. MUW 1, 1437 I A 30 (Joh. Schachen de Sancto Lamperto)

- Schaithaimer 1439 I 1 (als b. a.). MUW?

- Schebler 1437 I 6. MUW 1, 1428 I A 19 (Joh. Schebler de Rotenman)

- plebanus in Schenck Maiori 1421 II 4. MUW 1, 1420 II H 39

- Schenkchenberger canonicus ecclesie Brixinensis 1412 I 15. MUW 1, 1412 I A 40 (Joh. Schemkenberge, can. Brixinensis)

- Schilher 1409 II 12. MUW 1, 1409 II R 25 (Joh. Schilcher de Lawffen)

- Schiltdorffer plebanus in Gastpoltshouen 1430 I 4. MUW 1, 1430 I A 29 (Joh. Schildorffer, plebanus in Chaspaltshofen)

- Scholym canonicus ecclesie kathedralis Wratislauiensis 1415 I 11. MUW 1, 1415 I S 12 (Joh. Scolini, can. Bratislauiensis) 
- de Schram plebanus in Münsterberg 1437 II 10. MUW 1, 1438 I H 11 (Joh. de Schrom)

- Screyber de Weyssenburga 1428 I 9. MUW 1, 1426 I R 67 (Joh. Schreyber)

- Scriptoris de Frankfordia 1413 II 1. MUW?

- de Seccouia 1415 I 3. MUW 1, 1413 I A 26 (Joh. Chamer)

- de Segswar 1406 II 13. MUW?

- Seld (Saeld, Selde) (de Wienna) 1406 II 18; lic. in decr. 1419 II 22; dr. decr. 1420 II 23; jur. Dekan 1425 I, 1429 I, 1437 I, 1439 II. MUW 1, 1400 II A 25 (Joh. Seld de Wyenna); Rektor 1422 I, 1428 I

- de Semelin de Vngaria 1422 II 14. MUW?

- Sensensmid 1438 II 13 (als m. a.). MUW 1, 1434 I R 18

- Sews bacc. in decr. 1434 II 9 (vielleicht identisch mit Joh. Ro̊tlini de Opauia, siehe dort). MUW 1, 1427 I H 24 (Joh. Sews de Oppauia)

- Sigel ordinis canonicorum regularium de Walsee 1412 II 4. MUW 1, 1412 I R 130 (Joh. Sigel de Walsee, can. regularis ordinis sancti Augustini)

- de Sindelfing 1430 II 12 (als m. a.). MUW 1, 1424 II R 16 (Joh. Heym de Gundelfingen)

- Sindrami (Sinderami, Syndrami) 1405 II 4; jur. Dekan 1406 II, 1409 I, 1411 II (als m. a.); 1415 II, 1420 I. MUW 1, 1387 I S 6? (Joh. Syndrami de Heylgenstad) oder 1388 I S 11? (Joh. Syndrami studens in iure canonico); Uiblein, AFA I, 538f.; Uiblein, AFTh 2, 670 (Ioh. Sindrami officialis curie Pataviensis)

- de Sitauia (Zitauia) (Finsterwald[er]) 1416 II 6; bacc. in decr. 1420 II 25; lic. in decr. 1422 I 27. MUW 1, 1416 I H 37 (Joh. Finsterwaldis)

- Sompol de Thodia 1416 II 1. MUW?
- Spêt de Papenheim 1412 II 11; bacc. in decr. 1420 I 18. MUW 1, 1412 II R 37

- Sponsberg de Wratislauia 1425 II 2. MUW 1, 1425 II H 48 (Joh. Sponsperck)

- Stadelmair 1433 I 8. MUW 1, 1432 I R 97 (Joh. Stadelmayr de Frisinga)

- Stainger de Monaco 1431 I 9. MUW 11438 I R 144? (Joh. Stóringer de Monaco)

- Stange de Gorlicz 1432 II 7. MUW 1, 1425 II S 5 (Joh. Stang de Gollricz)

- Stark plebanus in Aldenpolan 1412 II 18. MUW?

- Stauffer de Plossenstauffen 1438 II 17. MUW 1, 1438 II R 78 (Joh. Staffer de Plossenstaffen)

- Stawdhaymer de O̊tting 1430 II 8. MUW 1, 1423 II R 36 (Joh. Stawthaymer)

- Streyperger canonicus Ratisponensis 1403 I 5. MUW 1, 1403 I R 37

- Stremphel de Weissemburga 1434 I 9 (als b. a.). MUW 1, 1430 I R 34

- Stro̊mberger plebanus ecclesie Sancte Marie Vallis Lauentine 1438 II 7. MUW 1, 1415 I R 6? (Joh. Stroberger de Dorffen)

- Stüxs de Weyden 1438 I 6. MUW 1, 1438 I R 2

- de Stukardia prepositus ecclesie collegiate in Boll 1418 II 13. MUW 1, 1418 II $^{1}$ R 10

- Sutoris de Freynstat presbyter Wratislauiensis diocesis 1437 II 2. MUW 1, 1437 II H 17 (Joh. Heinrici Sutoris, studens iuris)

- Sweydnitz canonicus ecclesie Sancte Crucis in Bradislauia 1410 I 13. MUW 1, 1410 I H 20

- de Sweinfordia 1419 I 18 (als m. a.). MUW 1, 1413 I R 1 (Joh. Himburg, baccalarius); Uiblein, AFA I, 531 (Joh. Hainburkch de Sweinfordia; 
Bruder von Gregorius H.; siehe diesen)

- Swerger de Sulcz 1413 I 28. MUW 1, 1410 II R 33 (Joh. Sweiker); Uiblein, AFA I, 538 (Joh. Swigher[r] [Schwiker])

- Tabernatoris 1416 I 26. MUW?

- Tagerhaim (Tagersheim, Tagersheym) 1411 I 11; 1413 I 12 (als m. a.) [beide Personen identisch?]; lic. in decr. 1414 I 30. MUW 1, 1411 I R 55 (Joh. Tagershaym de Renig); Uiblein, AFA I, 540

- de Tapolcza (Tapoltza) canonicus ecclesie Waradiensis 1434 I 1 (als m. a.); bacc. in decr. 1436 I 5; lic. in decr. 1438 I 12; dr. decr. 1438 I 17. MUW 1, 1423 II H 30 (Joh. Literati de Tapocza)

- Telkener de Cremniczia 1432 II 5 (als b. a.). MUW 1, 1426 I H 29 (Joh. de Chremnicz)

- Tornaw de Lutko (Lücko) 1411 II 10; bacc. in decr. 1420 II 26. MUW 1, 1418 II $^{1}$ S 4? (Joh. Dornaw de Lusacia)

- Traboldi de Bruxella 1411 II 3. MUW 1, 1411 I R 96 (Joh. Trabolt de Prußella)

- Turndl 1407 II 5 (als lic. a.). MUW 1, 1402 I R 18? (Joh. Türndel de Schalchdorff); Uiblein, AFA I, 541? (Ioh. Türndl); Uiblein, Mittelalterliches Studium, 126

- Tullinger 1418 II 12. MUW 1, 1410 I R 11? (Joh. Tullinger de Patauia)

- Turinge de Prawnsperg plebanus in Osterror 1413 I 24. MUW 1, 1413 I S 8 (Joh. Thuringi de Prawnsperg, plebanus in Ostỹrod)

- Vbelman de Lantzhuta 1416 I 3. MUW 1, 1416 I R 70 (Joh. Vbelman de Lanczhauta)

- de Westfalia (Westualia, Westualus) (Schallermann) (de Suzato) 1411 I 16 (als dr. decr.); jur. Dekan 1412 I (als m. a.), 1416 II, 1421 II. MUW 1, 1411 I S 1 (Joh. Schallermman dr. decr.); Uiblein, AFA I, 541 (Ioh. de Westfalia [de Saxonia]); Uiblein, AFTh 2, 670 (Ioh. Schalerman de Suzato); Schrauf, AFM 1, 108 (Ioh. de Westualia, rector)

- Wilhelmi de Corona 1424 II 2 (als m. a.). MUW 1, 1418 I H 40

- Wingler de Medling 1432 II 6 (als b. a.). MUW 1, 1429 I A 8 (Joh. de Medling)

- Winterburg 1413 II 4. MUW 1, 1413 I R 103; Uiblein, AFA I, 542 (Joh. de Winterberg)

- de Wincz plebanus in Foro Ruthenorum 1431 II 12 (als m. a.). MUW 1, 1417 II H 14? (Joh. de Wincz) oder 1420 I H 30? (Joh. Tanhawser de Wints)

- Wirnt 1406 II 16. MUW?

- Wolflein plebanus in Lind 1410 I 9. MUW 1, 1410 I R 29 (Joh. Wolfflein de Hilpurgehausen, plebanus in Lyndt)

- Wogawer 1408 I 8. MUW 1, 1389 I S 12 (als b. a.); Uiblein, AFA I, 542 (Joh. [Wogauer])

- Wolf de Wartemberg 1411 I 6. MUW 1, 1411 I H 42 (Joh. Wolf de Wartenwerg)

- Woluisheim de Waldbrunn 1412 II 12. MUW 1, 1411 I R 44 (Joh. Wolfweshaym de Waldeprunn); Uiblein, AFA I, 525 (Joh. de Baldeprünn [Waldbrunn]); Uiblein, Mittelalterliches Studium, 123

- Wucherl de Lewben 1418 II 22. MUW 1, 1417 II A 6 (Joh. Wucherl de Lewben)

- Wurcher de Vlma 1421 II 15 (als m. a.). MUW 1, 1417 I R 79 (Joh. Wurcher)

- de Zagrabia 1426 II 14 (als b. a.); dr. decr. 1432 I 17. MUW 1, 1424 II H 12? (Joh. Martini de Zagrabia)

- Czap de Bechthem 1429 I 12 (als m. 
a.). MUW 1, 1425 I R 62 (Joh. Czap — archidyaconus de Cibinio 1406 II 12. de Bechein)

- Czeller 1432 II 3 (als m. a.). MUW 1, 1423 I R 122 (Joh. Czeller de Augusta); Schrauf, AFM 1, 108 (Mag. Ioh. Zeller de Augusta, doctor med.); Schrauf, AFM 2, 261 (Mag. Ioh. Zeller de Augusta, art. et med. doctor, physicus Ladislai Regis)

- Czepeck (Zepek) (de Laybaico) 1413 I 13; bacc. in decr. 1431 II 25 (als plebanus ad Sanctum Vitum prope Laybacum). MUW 1, 1406 I A 8 (Joh. Czepek de Lebaco); Uiblein, AFA I, 532; Uiblein, AFTh 2, 673 (Ioh. Czepeck procurator prepositi [ad s. Stephanum Wienne])

- canonicus ecclesie Beati Martini in Cepus 1439 I 3. MUW? MUW?

- Zimischem archidiaconus de Pankata canonicus Agriensis 1413 I 25. MUW 1, 1413 I H 56 (Joh. de Zemsche)

- Zink 1413 II 13; (imm. gemeinsam mit seinem famulus Johannes Redler, siehe diesen). MUW 1, 1412 I A 12 (Joh. Czynk de Wienna); Schrauf, AFM 1, 108 (Mag. Ioh. Zink)

- de Znoyma altarista 1417 I 13. MUW 1, 1412 I H 45 (Joh. de Czama); Uiblein, AFA I, 542 (Joh. de Zama [Znoyma])

- (Czopot) plebanus in Nouoforo (prope Frisacum) 1429 I 5; bacc. in decr. 1431 II 22. MUW?

- Czwinger de Basilea 1424 I 13. MUW 1, 1423 I R 28 (Joh. Zwinger)

Ladislaus

- de Hederfar cantor et canonicus ecclesie Vesprimiensis 1434 II 1. MUW 1, 1430 I H 19? (Ladisl. Gregorii de Hechegdra)

- de Macedonia 1418 II 14. MUW 1, 1416 I H 30 (Ladisl. de Massidonio)

- de Marcellis prepositus Chanadiensis 1419 I 8. MUW 1, 1417 I H 40 (Ladisl. filius Petri de Marcelli)

- de Schlak canonicus ecclesie Baradiensis 1427 II 5 (als b. a.). MUW?

- de Strig (Strigh) (archidiaconus ecclesie Transsiluanensis) 1426 I 8; bacc. in decr. 1428 I 14; lic. in decr. 1431 II 17; dr. decr. 1436 II 14. MUW 1, 1413 I H 69? (Ladisl. Petri de Strig)

- de Czschach 1431 I 13. MUW 1, 1430 I H 55 ? (Ladisl. de Czaser)

Lambertus (Lampertus)

- de Rukhendorf 1441 II 1. MUW 1, 1436 I A 40 (Lamb. Rukkendorffer)
Laurencius

- Kasla de Wratislauia 1415 I 6. MUW 1, 1415 I H 44 (Laur. Gosola de Wratislauia)

- de Corona (de Septemcastris) 1429 I 10 (als m. a.); bacc. in decr. 1430 II 32. MUW 1, 1411 I H 11 (Laur. Jacob de Corona); Uiblein, AFA I, 544 (Laur. de Corona [de Septemcastris]); Schrauf, NH, 455 (Laur. de Corona, mag. et proc. $1430 \mathrm{I}$ )

- Lewbner filius Nicolai de Nissa 1431 II 6. MUW 1, 1431 II H 18 (Laur. Leybner de Nissa)

- de Obernburg (Obernburk, Oberngurkch) 1431 I 7 (als m. a.); bacc. in decr. 1433 II 13; lic. in decr. 1436 II 10. MUW 1, 1421 II A 15 (Laur. de Obergurg)

- de Rota plebanus in Kirchdorff 1418 I 18. MUW 1, 1417 II R 43 (Laur. de Rot) 
- Stephani de Corpona 1420 I 7. MUW 1, 1420 I H 17

Leo

- Geysenuelder de Ratispona 1420 II 19 (als m. a.). MUW?

Leonhardus (Leonardus, Lienhardus)

- Kellner de Wirgil 1435 I 4. MUW 1 , 1427 I R 9(Leonh. Kelner de Werigel)

- de Voytzperg 1414 I 18. MUW 1, 1414 I A 41 (Leonh. Voyczsperger)

- Gruber plebanus in Sweygkers 1420 I 4. MUW?

- Layminger canonicus ecclesie Patauiensis 1407 II 6. MUW 1, 1407 II R 36 (Leonh. Lamynger, can. Patauiensis)

- Meylestorffer 1418 II 1. MUW 1, 1414 II A 3 (Leonh. Meylestorffer de Seyttensteten)

- Prost canonicus ecclesie Ratisponensis 1407 II 1. MUW 1, 1407 II R 19 (Leonh. Probst)

- Rastaker de Villaco canonicus Inticensis 1421 I 15. MUW 1, 1411 I A 27? (Leonh. de Villaco)

- de Ruffo Monte 1402 I 10. MUW 1, 1402 I H 4

- Schawer (Schawr) 1405 II, 1406 II (als dr. decr.). MUW 1, vor $24 \mathrm{VI}$ 1377, 15 (Leonh. Schaur); 1394 I (Rektor Univ. Wien); Uiblein, AFA I, 544 (Leonh. Schawer)

- Scho̊ntrit de Salczburga 1438 II 2. MUW 1, 1438 I R 19

- prope scolas iuris[tarum] 1427 I 10. MUW?

- Selgerềt de Mò̀tnicz 1414 II 4. MUW 1, 1414 II A 5 (Leonh. Selge- ret de Montnich)

- de Stain Carniole 1441 I 4 (als m. a.). MUW 1, 1429 I A 7 (Leonh. de Stain)

- de Stayn 1423 II 4. MUW 1, 1421 I A 19 (Leonh. de Stain)

- Tenniger de Monaco Ordinis Minorum 1429 II 22. MUW 1, 1429 II R 52 (Leonh. Teninger de Monaco OFM)

Leutherus

- de Slesia 1427 I 5. MUW?

Liebhardus

- de Ratispona plebanus in Frễtinga 1416 II 32. MUW 1, 1394 II R 4? (Lieph. de Ratispona); Uiblein, AFA I, 545? (Liebh. de Ratisbona)

Lucas

- Slitt de Prün 1426 II 12 (als m. a.). MUW 1, 1421 I A 3 (Lucas Slit de Prün)

Ludowicus (Lodowicus, Ludovicus)

- de Herczogemburg 1423 I 8. MUW 1, 1410 II A 2 (Lodovicus Gôssl can. Herzogwrgensis); Uiblein, AFA I, 545 (Ludw. can. reg. de Hertzogenburga); Uiblein, Mittelalterliches Studium, 126

- Leupolt de Monaco bacc. in decr. 1442 I 15 (als m. a.). MUW 1, 1432 I R 91 (Ludow. Leopoldi de Monaco)

Luthardus (Lluthardus)

- de Chonstatt 1429 II 25. MUW 1, 1429 II R 59 (Luth. de Constat)

Luwertus

- Lankow de Danczk 1424 I 15 (als m. a.). MUW 1, 1424 I S 16 (Mag. Lowart. Lanko)
Manussius

- rector ecclesie in Vodwat bacc. in decr. 1423 I 13. MUW 1, 1422 I H 6 (Maynussius, plebanus in Wodyerad)
Marcus

Enenkel de Albrechtzperg canonicus Patauiensis 1416 I 17. MUW 1, 1412 I A 1 (Marc. Enenkchel) 
Lessna de Maiori Glogouia 1434 I 8. MUW 1, 1434 I H 28 (Marc. Lesna de Glogouia maiori)

Marquardus

- de Messenpach canonicus Patauiensis 1416 II 11. MUW 1, 1410 I A 27 (Marc. Messenpekch)

Martinus

- Back de Gamundia 1410 II 4. MUW?

- de Chranach 1412 II 19 (als m. a.). MUW 1, 1412 I R 116 (Mag. Mart. Ulrici Ekhardi de Statkchranach)

- in Habelswerd plebanus et canonicus Sancte Crucis Wratislauiensis 1420 II 13. MUW 1, 1420 II H 37

- Helman plebanus in Monte Petri 1416 II 29. MUW 1, 1416 II H 27

- Hublinger de Wels 1415 II 14 (siehe Georius de Rattaw). MUW 1, 1415 II R 67 (Mart. de Wels)

- archidiaconus de Hunyad ecclesie Transsiluanensis 1428 II 8; bacc. in decr. 1430 I 32. MUW 1, 1428 II H 30 (Mart. de Huniad)

- de Leibitz 1429 II 3 (als m. a.). MUW 1, 1420 II H 24 (Mart. de Lewbicz)

- de Oyta 1412 II 2. MUW 1, 1412 II S 1

- Petirbicz mansionarius ecclesie Wratislauiensis 1412 I 10. MUW 1, 1411 II H 30 (Mart. Peterwicz de Wratislauia)

- Phaffenhofer de Sancto Floriano procurator scole iuristarum 1427 II 7. MUW 1, 1422 II A 18? (Mart. Molitoris de Sancto Floriano)

- plebanus in Pơlan 1442 I 3. MUW?

- Pratesser 1430 II 18. MUW 1, 1417 II A 14 (Mart. Pratesszer de Wyenna)

- de Ratispona 1416 II 33. MUW 1, 1416 II R 33 (Mart. de Ratispana)

- Ro̊chel 1411 I 12. MUW 1, 1404 I R 12? (Mart. Reichlein de Argentina); Uiblein, AFA I, 546f.? (Mart. Rochel
[Rochl, Ro̊chli]); Uiblein, Mittelalterliches Studium, 126

- de Walthawsen (Walthausen, Walthusen) canonicus regularis 1419 I 12; bacc. in decr. 1425 II 8; lic. in decr. 1428 II 13; dr. decr. 1430 II 34; jur. Dekan 1438 II, 1440 I, 1442 I. MUW 1, 1417 I A 20 (Mart. ordinis canonicorum regularium s. Augustini de Walthusen)

- Wanislant de Rosenheim 1416 II 17. MUW 1, 1417 II R 39? (Johannes ! Waninßlandt de Rosenheym)

- de Wolfpach 1422 I 14 (als b. a.). MUW 1, 1412 I A 3 (Mart. Sebek de Volfpach); Uiblein, AFA I, 547 (Mart. Sebek)

- de Wolkersdorf 1435 II 3. MUW?

- Czeller de (Grewätzensteten) 1423 I 12; bacc. in decr. 1431 I 14. MUW 1,1417 I A 18

Maternus

- Tewfel de Brunna 1440 II 2. MUW?

Mathias (Matheus)

- Andree de Lyppa 1441 I 7. MUW 1, 1436 I H 17? (Math. Andree de Maczalaka)

- Beschorn de Noua Ciuitate 1421 II 8. MUW 1, 1415 I H 21? (Math. de Noua Ciuitate alias de Vinczho)

- Cordobok de Gora 1411 I 4. MUW 1, 1411 I H 7 (Math. Cordebok de Gora)

- de Kulled 1426 II 18. MUW?

- de Gnyznå 1418 I 22. MUW 1, 1418 I H 27? (Math. Virswent de Polonia)

- de Hermansdorff famulus 1420 II 5. MUW 1, 1419 I H 9 (Math. Los de Glogouia maiori, famulus suus (siehe Johannes Lemberg de Vratislauia)

- Pernicz (Bernicz) (de Maydburg) (Saxo) 1416 I 25; bacc. in decr. 1417 I 9. MUW 1, 1416 I S 6 (Math. Pernicz)

- de Quinqueecclesiis 1423 I 15 (als b. a.). MUW 1, 1420 II H 18 
- Regelshofer (Regelshoffer, Regelshouer) (de Herriden) 1408 I 3; lic. in decr. 1410 I 18; jur. Dekan 1419 I. MUW 1, 1395 II R 6 (Math. Chunradi de Herriden); Uiblein, AFA I, 547 (Math. Reglishower); Uiblein, AFTh 2, 681 (Math. Regelshofer)

- Schrat plebanus in Pechstal 1430 I 21. MUW?

- Slick prepositus Boleslauiensis ecclesie collegiate 1428 II 3; bacc. in decr. 1430 II 31. MUW 1, 1426 I H 17 (Matheus Slichk de Egra, can. in Prunn)

- Stephani de Soldino 1416 II 8. MUW?

Michael (Michahel, Michel)

- de Åczmanspach bacc. in decr. 1402 I 8; lic. in decr. 1407; jur. Dekan 1408 II, 1410 II, 1414 II. MUW? Uiblein, AFA I, 547

- de Boden canonicus ecclesie Transsiluanensis 1430 II 5. MUW 1, 1426 I H 4 (Mich. de Bodann)

- Klingenstainer 1437 I 18. MUW 1, 1434 I R 4 (Mich. Chlingenstain de Salczburg); Schrauf, AFM 2, 252 (Mag. Mich. de Klingenstein, rector univ.)

- Ekhart de Ardisch de Septemcastris 1434 I 10. MUW 1, 1434 I H 34

- Falkonis arcium mag., decretorum bacc. et dr. in medicina 1417 II 13. MUW 1, 1418 II $^{2}$ Rektor Univ. Wien (Mich. Falkonis arcium et medicine dr., decr. bacc. et plebanus in Tyfer); Schrauf, AFM 1, 104 (Mag. Mich. Falkonis, decretor. bacc. et doctor med. Montis Pessulani, plebanus de Tifer necnon magnif. dom. Hermanni comitis Cilie etc. consiliar. et phyzicus)

- plebanus in Villa Jacobi de Septemcastris 1437 II 6. MUW 1, 1438 I H 5

- plebanus in Villa Thobie bacc. decr. 1437 II 19. MUW?
- Freyleich 1438 I 1. MUW?

- de Go̊pping 1424 II 1 (als m. a.). MUW 1, 1412 R 4 (Mich. Harsch de Göppinngen)

- de Herbelstat 1412 I 17. MUW?

- de Holabrunna 1425 I 6. MUW?

- de Insula Christiana (de Septemcastris) plebanus 1418 II 26; bacc. in decr. 1438 II 18. MUW 1, 1418 II $^{2}$ H 8 (Mich. de Magna Insula)

- de Lechling 1414 I 4. MUW 1, 1414 I R 23? (Michel Bechlinger de Bechling)

- Liebhardi plebanus in Waldarn 1419 I 23. MUW?

- Moschicz 1420 II 9 (als m. a.). MUW 1, 1420 II H 32? (Mag. Michel Nosticz)

- rector ecclesie in Napelstorff 1421 I 10. MUW 1, 1421 I A 21 (Mich. plebanus in Napelstorff)

- Phêffel de Ingolstat 1418 II 24. MUW 1, 1418 II $^{1}$ R 52

- de Priuidia 1430 II 11; bacc. in decr. 1434 I 17; lic. in decr. 1435 II 17. MUW 1, 1422 I H 21

- de Rẻdisch altarista in Praga 1424 II 9. MUW 1, 1424 II A 12 (Mich. quondam Petri de Redisch)

- Regelzhofer 1413 II 6. MUW 1, 1413 I R 127 (Mich. Regelshofer plebanus in Ghay)

- (Jeskonis) de Resinkirche (Rosenkirch) $1411 \mathrm{I} 1$; bacc. in decr. $1413 \mathrm{I}$ 19. MUW 1, 1411 I H5 (Mich. Jeschkonis de Risenkirch)

- de Sancto Ypolito canonicus regularis 1408 II 2. MUW?

- de Schrick 1424 I 6 (als m. a.). MUW 1, 1417 I A 22? (Mich. Puff de Slichk) oder 1417 I A 25? (Mich. Dietmari de Schrick); Schrauf, AFM 1, 106 (Mag. Mich. Puff de Schrick, doctor med.); Schrauf, AFM 2, 256 (Mag. Mich. Puff de Schrichk, art. et med. doctor) 
- Stankonis de Wratislauia 1426 I 7. MUW?

- Steger 1432 I 3; bacc. in decr. 1438 II 19. MUW 1, 1426 I A 10

(Mich. Steger de Stira)
- (Heylsmar, Hylsmar) de Stolczenberg 1418 II 16; bacc. in decr. 1422 I 29; lic. in decr.1435 I 12; dr. decr. 1435 II 12; 1435 II 16 (ad structuram facultatis 6 fl.). MUW?
Narcissus

- Hercz 1410 I 7. MUW 1, 1406 II R 7 (Narc. Hercz de Berching); Rektor Univ. Wien 1423 I. Uiblein, AFA I, 549; Uiblein, Mittelalterliches Studium, 127; Uiblein, AFTh 2, 684; Schrauf, AFM 1, 105 (Narc. [Herz], notarius universitatis); Schrauf, AFM 2, 254 (Mag. Narc. [Herz], vicarius eccl. s. Stephani)

Nicolaus (Nycolaus)

- Andree de Strêlen 1414 II 14 (als m. a.). MUW 1, 1414 II H 16

- de Asparn 1420 I 10. MUW 1, 1417 II A 28

- Atyser de Villa Las 1415 II 3. MUW 1, 1400 I H 9? (Nic. de Villa sancti Ladislai)

- Bethneri canonicus regularis monasterii Beate Virginis Wratislauie in Arena 1433 II 5 (siehe auch Sigismundus Aczo). MUW 1, 1434 I H 4 (Mag. Nic. ord. s. Augustini canonicorum regularium)

- Bodo 1435 II 4. MUW?

- Kalman de Hoczemplocz 1423 I 4. MUW 1, 1423 I H 12 (Nic. Colomanni de Hoczenplocz)

- de Chasma (Chasmensis) 1416 II 19; bacc. in decr.1420 I 17; lic. in decr. und dr. decr. 1422 I 24 (als can. Chasmensis). MUW?

- Kynsperger de Chrewsen 1430 II 14. MUW 1, 1430 I R 55 (Nic. Kunisperger de Chreusen)

- Kitzka (Kyczka, Kitschka) plebanus in Pleusnicz 1411 II 17; bacc. in decr. 1413 I 30; lic. in decr. 1419 II 15.
MUW 1, 1411 II H 31 (Nic. Kiczka, plebanus in Plausenicz)

- Clementis 1418 II 28. MUW 1, 1418 I H 19 (Nic. Clementis de Megesfalva)

- Cluting (Gloting) (de Traiecto) 1419 I 22; bacc. in decr. 1423 I 31. MUW 1, 1419 I R 62 (Nic. Clòting de Traiecto inferiori, iurista)

- Clericus de Adorian 1416 I 21 (als m. a.). MUW 1, 1410 II H 3 (Nic. de Adrian); Uiblein, AFA I, 549 (Nic. Clericus de Adrian)

- Ko̊schel de Clawsemburg 1414 II 3 (als m. a.). MUW 1, 1401 I H 16 (Nic. de Septemcastris de Clausenburch); Uiblein, AFA I, 549 (Nic. de Clausenburg [de Septemcastris])

- Krềt 1410 I 11. MUW?

- Cristanni de Reddin 1420 I 2. MUW 1, 1419 I S 1 (Nic. Cristanni de Radden)

- Krotendarffer 1410 I 6 (als m. a.). MUW 1, 1398 II A 37 (Nic. Crotendorffer de Noua Ciuitate); Uiblein, AFA I, 549; Uiblein, Mittelalterliches Studium, 127

- Ecker 1416 II 7. MUW 1, 1411 I A 18 (Nyc. Ekker de Brixna); Uiblein, AFA I, 550 (Nic. Ekkär)

- Erlacher de Purkhawsen (Purkhusa) 1431 II 5; bacc. in decr. 1435 I 8. MUW 1, 1431 I R 17 (Nic. Erlacher, pedagogus)

- Vogel de Wratislauia 1412 I 3. MUW 1, 1410 I H 12 (Nic. de Wratislauia); Uiblein, AFA I, 550 (Nic. de Voratislavia) 
- Glocz (Stürpecher) 1434 I 7 (als m. a.); bacc. in decr. $1440 \mathrm{I} 5$; lic. in decr. 1440 II 12. MUW 1, 1424 II H 16 (Nic. Stürczenpecher de Glacz)

- Gramus Wratislauiensis ac Olomucensis ecclesiarum canonicus 1422 II 1. MUW 1, 1422 II H 1

- Graudencz 1411 I 14. MUW 1, 1411 I S 4 (Nic. de Grudens)

- Hêppel de Grassaw 1420 II 7. MUW 1, 1415 I R 68

- Heinrici de Praga archidiaconus Pragensis 1424 II 8. MUW 1, 1424 II H 29 (Nic. Hinrici, archidiac. Pragensis)

- de Helta 1416 II 30 (als m. a.). MUW 1, 1407 I H 3 (Nic. de Heltaw); Uiblein, AFA I, 551 (Nic. de Helta)

- Hermanni de Fridlang 1424 I 16. MUW 1, 1424 I S 17 (Nic. Hermanni de Fredlannd)

- Heroldi de Frawnstat canonicus et scolasticus Glogouiensis Maioris 1419 II 1. MUW 1, 1419 II $^{2} \mathrm{H} 3$ (Nic. Heroldi de Frawenstat)

- Ysenhütel de Wienna 1413 II 11. MUW 1, 1413 II A 17 (Nic. Eysenhutel de Wienna)

- Jenkebicz de Woslawicz 1429 I 4. MUW 1, 1428 II H 29 (Nic. Jencowicz de Poslowicz)

- Johannis de Dacia 1420 II 10. MUW 1,1420 II S 7

- Johannis de Frigido Fonte 1418 I 6. MUW 1, 1418 I H 9 (Nic. de Fridefuncte[sic!])

- Lepes archidiaconus et canonicus ecclesie Transsiluanensis 1434 II 2 (als m. a.); bacc. in decr. 1437 II 17; lic. in decr. 1439 II 6; dr. decr. 1439 II 8. MUW 1, 1426 II H 10 (Nic. Leppes de Waruskezi)

- Martini de Brůnsperg de Prussia 1436 I 3. MUW 1, 1434 I S 3 (Nic. Martini de Brawnsperg)

- Mathie Goldperg (Goltperg) de
Wratislauia 1410 I 14; bacc. in decr. 1416 II 38. MUW 1, 1410 I H 21

- Mayr rector capelle in Weyding 1421 I 12. MUW?

- Micheler 1419 II 10. MUW 1, 1419 II $^{2}$ H 11 (Nic. Micheler de Lebin)

- Paskowicz plebanus in Kant 1426 II 5. MUW 1, 1424 II H 9? (Nic. Paskowicz de Steynauia)

- amicus decani Patauiensis canonicus ecclesie Wratislauiensis 1413 I 33. MUW?

- Peck de Nyssa 1424 I 9. MUW 1, 1423 II H 34 (Nic. Pekch de Nissa)

- Perold canonicus Brixinensis 1403 I 2;1411 II 18 (als Dompropst von Brixen; nochmals imm.?). MUW 1, 1403 I A 6 (Nyc. Perolt, can. Prixinensis, plebanus in Matrey); Uiblein, AFA I, 549 (Nic. [Berolt] prepositus Prixnensis)

- de Průmea 1413 II 7. MUW 1, 1413 I R 113 (Nic. de Prumea, b. a.); Uiblein, AFA I, 552 (Nic. de Prumea)

- de Prussia 1409 II 1. MUW 1, 1409 I S 6? (Nic. Karin de Gdancz)

- Rebiczer canonicus Ratisponensis 1432 I 9. MUW 1, 1432 I R 81 (Nic. Redewicz, can. Ratisponensis)

- Reiberstorffer de Montibus Chutnis 1412 II 9. MUW 1, 1412 II H 7

- Reychel de Wratislauia 1433 II 1. MUW 1, 1428 II H 6 (Nic. Reychl de Bratislauia)

- Reyman de Olsna 1429 II 18 (als m. a.). MUW 1, 1429 II H 34 (Mag. Nic. Reyman de Olsen, in artibus)

- de Rogettel 1409 II 5. MUW 1, 1409 I S 10 (Nic. Gunterin de Rogetel)

- plebanus in Růsdarf 1409 II 9. MUW?

- Salczmesser de Frankfordia circa Oderam 1413 II 18 (als m. a.). MUW 1, 1414 I S 1 
- plebanus ad Sanctum Vdalricum in Noua Ciuitate extra muros 1431 II 9. MUW?

- Sartoris 1411 I 15. MUW?

- Sartoris de Gora 1411 I 7. MUW 1, 1411 I H 16

- Satler de Kunigsberg 1441 I 6 (als m. a.). MUW 1, 1436 I S 1 (Nic. Sellatoris de Kunigsperg)

- Schönberg (Schho̊nperger) de Olmüncz 1428 I 7; 1431 II 21 bacc. in decr. MUW 1, 1428 I H 1? (Nic. de Schenberck, can. Brumensis)

- de Seydlicz plebanus in Nouoforo etc. 1411 II 5. MUW 1, 1411 II H 1 (Nic. de Seydlicz, plebanus in Nouoforo, can. eccl. sancte Crucis Wratislauie)

- Speyser 1409 I 9; 1422 I 4 bacc. in decr. (als plebanus in Meyssilting). MUW 1, 1394 II R 2? (Nic. Spyser de Constancia)

- Stock (Stok, Stokk, Stokch) de Glogouia (Maiori) 1413 I 17; bacc. in decr. 1421 I 20 (als m. a.); 1422 I 25 lic. in decr. und dr. decr. (als precentor cripte Sancte Crucis in summo Wratislauiensis); 1422 II jur. Dekan. MUW 1, 1413 I H 33 (Nic. Stock de Maiori Glahouia); Uiblein, AFA I, 553 (Nic. Stok de [Maiori sive de Magna] Glogovia); Uiblein, Mittelalterliches Studium, 127

- Stubner (Stúbner, Stůbner ) canonicus Agriensis 1430 I 11; bacc. in decr. 1433 I 12; lic. in decr. 1435 I, 13; dr. decr. 1435 II 13. MUW 1, 1422 I H 7? (Nic. Stubner de Kremnicia)

- [Swarat] (erscheint in der Quelle als decanus Brixinensis; identifiziert nach Santifaller, Brixner Domkapitel, 484f., Nr. 338) 1420 I 21 bacc. in decr.; lic. in decr.; dr. decr. MUW 1 , 1395 I H 22 (Nyc. Swarat de Wratislauia)

- de Themezwar 1418 I 8 (als m. a.); bacc. in decr. 1421 I 17; lic. und dr. decr. 1422 I 26. MUW 1, 1411 II H 13 (Nic. de Temeschwar); Uiblein, AFA I, 553 (Nic. de Themesswär)

- canonicus ecclesie Transsiluane et plebanus in Tartala 1413 I 2 (als m. a.). MUW? Schrauf, NH, 508, 521 (Nic. Wydener, can. eccl. Transsilv., pleban. in Tartlaw, mag. et proc. $1414 \mathrm{I}$ )

- plebanus in Walthuden 1406 II 11. MUW 1, 1406 II H 4 (Nic. de Waltudia)

- de Waradino 1414 I 7; bacc. in decr. 1417 II 2; lic. in decr. 1419 II 23; dr. decr. 1421 I 19. MUW 1, 1411 II H 3

- Weinschenk de Rudlinga 1418 II 2 (als m. a.). MUW 1, 1412 II A 38 (Nic. Weinschenkch de Rudlinnga); Uiblein, AFA I, 552 (Nic. de Rüdlingen [Rütlinga])

- Wenke canonicus ecclesie Vratislauiensis, prepositus Othmuchouiensis: als bacc. in decr. imm. 1422 II 2; bacc. in decr. (?) und lic. in decr. 1423 II 9. MUW 1, 1422 II H 22 (Nic. Wenck, prep. eccl. collegiate Otmochinensis et can. Wratislauiensis)

- Wyel de Wlessna 1418 I 1. MUW 1, 1418 I H 1 (Nic. Wyel de Bleschna)

- Wolf de Wartemberg 1419 II 4; bacc. in decr. 1422 I 9 (siehe Conradus Wolff). MUW?

- Wolff de Wartemberg 1411 I 5. MUW?

- de Zagrabia 1433 I 9 (als m. a.). MUW 1, 1423 I H 1

- Zedlicz canonicus ecclesie Bregensis 1418 I 17. MUW 1, 1418 I H 37 (Nic. Zedlicz., can. eccl. Bregensis Wratislauiensis diocesis)

- Czink quondam prepositus in Varaw 1433 I 1. MUW 1, 1433 I A 11 (Nic. Czink, professus ord. s. Augustini canonicorum regularium monasterii in Voraw, olim prepositus ibidem) 
Oliverus

- de Ymmerzel 1438 I 5. MUW?

Oswaldus

- plebanus in Korkyngen 1402 I 11. MUW 1, 1402 II R 28 (Osw., rector eccl. parrochialis in Körkingen)

- Holer de Valle Eni 1421 II 10. MUW 1, 1417 II A 8

- de Rozgon prepositus Agriensis 1437 I 3. MUW 1, 1437 I H 33

- de Salina 1418 I 19. MUW 1, 1415 II R 44; Uiblein, AFA I, 554

(Osw. Rotel de Salina)
- canonicus Salzburgensis, comes de Artenberg 1421 I 1. MUW 1, 1421 I R 1 (Osw., comes de Artenberg, can. et professus eccl. metropolitane Salczburgensis)

- Strawss de Ratispona 1419 I 5. MUW 1, 1418 II $^{1}$ R 8 (Osw. Strawß de Ratispona)

Otto

- Seinreich 1411 II 14. MUW 1, 1409 I R 17 (Otto Seynrich de Monaco)
Pangracius

- Conradi de Sweinbart 1430 I 6. MUW 1, 1420 II A 5 (Pangr. Linifex de Sweynwart)

Paulus

- de Apoldia Inferiori presbyter 1435 II 5 (als b. a.). MUW?

- (de Sana) abbas de Bata 1414 I 16; bacc. in decr. 1417 II 3. MUW 1, 1414 I H 1 (Paul. abbas monasterii beati Michaelis archangeli de Bata OSB); Uiblein, AFA I, 555 (Paulus [de Sana] abbas de Watha [de Ungaria])

- de Gleybicz 1436 I 1 (als b. a. Krakau); bacc. in decr. 1438 II 20; lic. in decr. 1442 I 8. MUW 1, 1436 I H 35 (Paul. Petri de Gleybitz)

- Glen de Noua Ciuitate 1431 I 8. MUW 1, 1431 I R 68

- Mankelwicz de Legnicz 1430 II 2. MUW 1, 1430 I H 15 (Paul. Maukelwicz de Legnicz)

- Meck de Ornbawr 1439 I 7. MUW 1, 1435 I R 16 (Paul. Mechk de Orenbawr)

- archidiaconus Symigiensis et canonicus Wesprimiensis 1415 I 1.
MUW 1, 1412 II H 54 (Paul. archidiac. Simidiensis)

- Syndrami de Nissa 1420 II 17; bacc. in decr. 1424 II 11. MUW 1, 1414 II H 29 (Paul. Sindram de Nißa)

- Troppawer de Wienna 1419 II 7 (als m. a.). MUW 1, 1412 I A 43

- (Pãwrl) de Wienna (Wyenna) 1409 II 11 (als m. a.); bacc. in decr. 1419 II 19; lic. in decr. 1419 II 20; dr. decr. 1420 II 22; jur. Dekan 1420 II, 1425 II, 1428 II, 1431 II. MUW 1, 1399 I A 7 (Paul. Pawirll de Wyenna); Uiblein, AFA I, 555 (Paul. de Wienna); Uiblein, AFTh 2, 690 (Paul. [Paurl] de Wienna); Schrauf, AFM 1, 108 (Dr. Paul. de Wienna)

Perchtramus

- de Veltheim 1416 I 27. MUW 1, 1416 I S 7 (Bertram. Veltheim)

Petrus

- Absolonis de Herdlof 1419 II 2. MUW 1, 1419 II² S 3 (Petr. Absolonis Herdloff)

- Agmanni dr. decr. 1430 II 35. MUW?

- de Alba Transsiluana (archidiaconus Transsiluanensis) 1420 II 14; bacc. in decr. 1423 I 28; lic. in decr. 
1425 II 6. MUW 1, 1416 II H 26?

(Petr. de Septemcastris) oder 1420 I H 32? (Petr. de Castro Scheges)

- Amsteg de Selgenstad 1415 II 4. MUW 1, 1410 II R 7 (Petr. Ingradu de Seligenstat); Uiblein, AFA I, 559 (Petr. de Selligenstat)

- de Arnsdorf 1409 II 7 (als m. a.). MUW 1, 1399 I A 28 (Petr. Perigochsel de Arnsdorff); Uiblein, AFA I, 558 (Petr. Pergexel [Bergextel, Berigåxl]); Uiblein, Mittelalterliches Studium, 128

- de Augusta 1409 I 8. MUW 1, 1389 II R 28 oder 1390 II R 2 (Petr. Eberhart de Augusta); Uiblein, AFA I, 555 (Petr. de Augusta)

- Bartholomei de Gơrlitz 1437 I 10. MUW 1, 1434 I S 4

- de Caldario de Lynss 1416 I 11. MUW 1, 1416 I R 35 (Petr. de Kaldario de Lyns)

- Cerdonis presbyter vicarius in Melsak 1420 II 16. MUW 1, 1393 I S 3? (Petr. de Mellsak)

- Kieler plebanus in Campidona 1429 II 21. MUW 1, 1429 II R 51 (Petr. Kråler, plebanus in Campidona)

- Chotter de Patauia 1416 II 12. MUW 1, 1416 II R 17 (Petr. Chocter de Patauia)

- Chramer plebanus in Anaso 1431 I 2. MUW 1, 1403 I A 15 (Petr. Institoris de Anazo); Uiblein, AFA I, 555 (Petr. Chramer de Anazo); Uiblein, Mittelalterliches Studium, 128

- Crombach de Pruszella (als m. a.) 1412 I 8. MUW 1, 1412 I R 2 (Mag. Petr. de Bruchsella)

- Deckinger (Dekinger; Dekchinger) (de Wynna) 1402 I 12 (als m. a.); lic. in decr. und jur. Dekan 1412 II und 1418 II (als Kanoniker von St. Stephan und Vizekanzler der Univ. Wien); dr. decr. 1419 II 18. MUW 1, 1387 II A 20 (Petr. Dekchinger);
Rektor 1399 I, 1404 I, 1410 I, 1414 I; Uiblein, AFA I, 555f. (Petr. Deckinger [Dek(k)kinger, Dekingger, Dechinger, Deklinger, Tegginger, Tekinger, Tenginger]); Uiblein, Mittelalterliches Studium, 128; Uiblein, AFTh 2, 691 (Petr. Deckinger [Dekchinger]); Schrauf, AFM 1, 104 (Petr. Deckinger, decretorum doctor et decanus cap.)

- de Valle Sancte Marie 1412 I 14. MUW 1, 1412 I H 22 (Petr. plebanus de Valle Marie)

- Ettenhayn de Argentina 1424 II 4. MUW 1, 1420 II R 7? (Petr. de Argentina) oder 1422 II R 54? (Petr. de Ettenheim)

- Etter 1413 I 14 (als lic. a.). MUW 1, 1391 II A 22 (Petr. Echer); Uiblein, AFA I, 556 (Petr. Etter de Wyenna)

- Fabri de Ostrauia 1428 I 2. MUW 1, 1428 I H 7

- Fuchs professus monasterii in Schussenried OPraem 1431 II 10; bacc. in decr.1433 II 11. MUW 1, 1429 II R 38 (Petr. Füschs de Marchdorff, professus OPraem)

- de Hêdersdorff 1412 I 11. MUW?

- de Hamelburg 1426 II 15 (als b. a.). MUW 1, 1413 II R 64

- Hebringer plebanus ad Sanctum Andream in Goss 1430 I 10. MUW 1, 1430 I A 32 (Petr. Hebringer, plebanus in Gôss)

- Hecht de Künigsperg 1413 I 1; bacc. in decr. 1416 I 29. MUW 1, 1412 II S 7 (Petr. Hecht de Koningesberg)

- de Heympach 1422 II 4 (als b. a.). MUW 1, 1415 II R 35 (Petr. Heymbach)

- Hekch de Reyspach bedellus theologie et medicine 1423 I 16. MUW 1, 1422 II R 75 (Petr. Heck, pronunc pedellus dominorum theologorum et medicorum); Schrauf, AFM 1, 106 (Petr., pedellus fac. med. [Petr. Heck de Reyspach]) 
- de Heltaw 1415 I 7. MUW 1, 1416 II H 26? (Petr. de Septemcastris)

- Henckel 1416 II 15. MUW 1, 1416 II R 20 (Petr. Henckel de Francfordia)

- de Herczogburga 1420 I 14. MUW 1, 1399 I A 19 (Petr. Pranpeck de Herczogenburga); Uiblein, AFA I, 557 (Petr. de Herczogenburga); Uiblein, Mittelalterliches Studium, 128

- Hoszil canonicus ecclesie collegiate in Anolspach 1417 I 2. MUW?

- Hủmel de Petouia 1439 I 2. MUW 1, 1439 I A 1

- (Newsidler) de Laa 1426 II 6; lic. in decr. 1432 II 12. MUW 1, 1423 I A 4 (Petr. Newsidler de La)

- de Lantshuta 1426 II 3 (als m. a.). MUW 1, 1418 II $^{1}$ R 53? (Petr. de Lantzhut)

- Mürbacher de Lincz 1426 II 2 (als m. a.). MUW 1, 1418 I A 14 (Petr. Mùrbuczel de Lincza); Uiblein, AFTh, 693 (Petr. [Murbuczel, Murbacher] de Linz [Lyncz])

- plebanus de Můschna de Septemcastris 1434 I 4. MUW 1, 1422 I H 2? (Petr. de Muschna)

- Nabok (Nobag, Nowag) de Nissa 1420 II 18; bacc. in decr. 1423 I 30; lic. in decr. 1428 I 10; dr. decr. 1428 I 12; jur. Dekan 1432 II (als can. ecclesie Transiluane). MUW 1, 1415 I H 57? (Petr. de Nyssa); Uiblein, AFTh, 693 (Petr. Naboch)

- Naffczer (Nafczer) de Wisenstaig (Wysenstaig) 1438 II 4; 1440 II 5 (zweimal imm.?); bacc. in decr. 1441 I 12. MUW 1, 1438 II R 16 (Petr. Nafczer de Wisenstaig)

- O̊denfurter de Liechtenburg 1431 I 4. MUW 1, 1423 II R 70

- Pachmülner (Pachmulner, Pachmulnår) (de Eberspewnt) 1419 I 21 (als $\mathrm{m}$. a.); bacc. in decr. $1422 \mathrm{I} 3$; lic. in decr. 1424 I 22; dr. decr. 1430 I 25; jur. Dekan 1424 II, 1431 I, 1434 II, 1438 I, 1441 II. MUW 1, 1411 II R 70 (Petr. Pachmulnår de Eberspewnt); Uiblein, AFA I, 556 (Petr. Eberspånd [Erberspand]); Uiblein, Mittelalterliches Studium, 128

- de Palocz 1431 II 2 (als m. a.). MUW 1, 1426 II H 1 (Petr. filius dom. Mathyus de Paloth, pro se et duobus famulis)

- plebanus de Pamenczina 1418 II 23. MUW 1, 1418 II $^{1} \mathrm{H} 20$ (Petr., plebanus in Paymanczina)

- Pauli de Alba Regali 1416 I 20 (als m. a.). MUW 1, 1396 I H 22? (Petr. de Alba Regali)

- de Pemeshaim 1430 I 19. MUW 1, 1412 I R 103? (Petr. Hermellinus de Benshaim)

- Pherlini 1409 II 2. MUW?

- Polcz plebanus in Marẻusch 1417 I 19. MUW 1, 1417 II A 1 (Petr. Polcz, plebanus in Maraucz)

- de Poseda 1439 I 4. MUW 1, 1438 II H 38 (Petr. de Posega)

- presbyter bacc. in decr.1430 II 28. MUW?

- de Rauenspurg 1432 I 12 (als m. a.); bacc. in decr. 1434 I 15. MUW 1, 1423 II R 60 (Petr. Wirtt de Rauerspurga)

- plebanus Sancti Georii super Ybsueld 1434 I 12. MUW?

- de Sancto Ypolito canonicus regularis 1420 II 21. MUW 1, 1420 II A 18

- plebanus Sancti Petri prope Judenburgam 1402 I 9. MUW 1, 1402 I A 21

- Sartoris de Kirchhain 1437 I 11. MUW 1, 1441 I S 17? (Petr. de Kirchhain, notarius domini officialis curie Patauiensis)

- Schenkch de Weybstat 1414 II 8. MUW?

- Sculteti de Russia 1423 I 6. MUW 1, 1423 I H 68

- Sesselman 1415 I 21. MUW 1, 
1412 I R 84 (Petr. Sesselman de Culmach)

- Stang de Ersperg 1416 I 4. MUW 1, 1415 II H 34 (Petr. Stang de Hersperg, plebanus in Reichenaw)

- de Sulczpach canonicus ecclesie Wissegradensis 1426 I 1; bacc. in decr. 1428 I 15 (als can. Sagrabiensis). MUW 1, 1421 II R 24? (Petr. de Sulczpach)

- Tegerpek de Scheyrn 1413 I 27 (als m. a.). MUW 1, 1407 II R 2 (Petr. Tegelpech de Schår); Uiblein, AFA I 559 (Petr. Tegerpekch [de] Schei[e]rn [Scheyrn])

- Teschner de Wratislauia 1411 I 13. MUW 1, 1411 I H 37

- de Toran 1420 I 13. MUW?

- Warnaw (Barna) (de Prusia) 1414 I 19 (als m. a.); bacc. in decr. $1416 \mathrm{I}$ 31; lic. in decr. 1416 II 36; dr. decr. 1416 II 40. MUW 1, 1414 I S 16 (Mag. Petr. Barno)

- de Weydenpach 1433 II 17. MUW 1, 1429 I H 45

- Cziller 1429 I 14. MUW?

- Zirnperger 1423 II 6. MUW?
Philippus (Phylippus)

- de Cronemberg canonicus Maguntinensis et Treverensis 1414 I 2. MUW 1, 1410 I R 43 (Phil. de Kronberg, can. Moguntinensis)

- Gladiatoris 1433 II 2. MUW 1, 1430 I A 27 (Phil. Gladiatoris de Wienna)

- (Dino) de Smolna 1412 I 2; bacc. in decr. 1417 II 5. MUW 1, 1411 II H 23 (Phil. de Smolna)

- Speyser 1408 I 4. MUW?

- Swarat de Brixina 1433 I 7; bacc. in decr. 1436 II 18; lic. in decr. 1440 II 13. MUW 1, 1430 I A 2

Pilgrimus

- de Zegenberg 1413 I 26 (als m. a.); bacc. in decr. und lic. in decr. 1418 II 6. MUW?

Procopius

- Stampen de Wratislauia 1421 II 19. MUW 1, 1413 I H 6 (Procop. Stamper de Wratislauia); Schrauf, AFM 1, 107 (Mag. Procop. Stampin de Wratislauia); Schrauf, AFM 2, 248 (Mag. Procop. de Wratislavia, bacc. med.)
Rabanus

- de Lybenstain canonic[us] ecclesie Maguntinensis 1430 I 16. MUW 1, 1430 I R 3 (Raban. de Lebenstain, can. eccl. Maguntinensis)

Reinhardus

- de Helmstat canonicus ecclesie Spirensis 1412 I 7. MUW 1, 1412 I R 1

Richardus

- Schlupff 1409 I 2. MUW?

- de Superiori Lapide canonic[us] ecclesie Maguntinensis 1430 I 17. MUW 1, 1430 I R 4 (Rich. de La- pide, can. eccl. Maguntinensis)

Rudolfus

- Bellazon, scolasticus et canonicus Curiensis 1411 II 7; bacc. in decr. 1414 I 29. MUW 1, 1411 II A 1

- decanus ecclesie Sancti Petri Basiliensis 1418 I 16. MUW 1, 1418 I R 34? (Rud. de Basilea, plebanus in Polan)

- Westersteter canonicus Augustensis 1412 I 19. MUW 1, 1412 I R 4 (Rud. de Westersteten, can. eccl. Augustinensis) 
Servatius (Seruacius)

- de Corona 1426 II 7. MUW 1, 1420 II H 19

- de Mergenburg 1424 II 6 (als m. a.). MUW 1, 1418 I H 7 (Seru. de Mergenburg)

Sigismundus

- Aczo familiaris eius 1433 II 6 (siehe Nicolaus Bethneri). MUW 1, 1430 II H 13 (Sig. Arcze de Sweydnitz)

- Flemming de Lantzhuta 1414 I 1. MUW 1, 1414 I R 15

- de Styra monasterii de Gersten 1425 II 1. MUW 1, 1423 I A 37 (Syg., professus in Gresten)

- Walich de Aybling 1426 II 17. MUW 1, 1427 I R 36? (Sig. Walich de Eyblinga)

- (de Septemcastris) plebanus in Wirthelm 1409 II 10; bacc. in decr. 1412 II 14. MUW 1, 1410 I H 7 (Sig. plebanus in Wirtalham)

- Cisterdarffer 1430 II 7. MUW 1, 1423 I A 39 (Sygm. Cistersdorffer)

Silvester (Sylvester)

- bacc. in decr. 1415 II 12 (identisch mit Folgendem?)

- Flieger de Augusta 1411 II 12 (identisch mit Vorigem?) MUW 1, 1411 I R 89 (Silu. Feyger de Augusta)

- Wager de Patauia 1416 I 14. MUW 1, 1411 I R 110? (Silu. de Patauia); Uiblein, AFA I, 562 (Silv. de Patavia) ; Uiblein, Mittelalterliches Studium, 129

Simon (Symon)

- Alberti altarista ecclesie Olmoncensis 1413 I 3. MUW 1, 1412 II H 56 (Sym. Alberti de Olmucza)

- de Altemburg 1421 II 7. MUW 1, 1421 II A 1 (Sim., professus OSB in Altenburga)

- de Asparn 1422 I 19 (als m. a.); bacc. in decr. 1440 I 9; lic. in decr. 1440 II 10. MUW 1, 1417 I A 1
- de Bacia Agriensis et Quinqueecclesiensis ecclesiarum canonicus 1430 II 6. MUW 1, 1430 II H 21

- plebanus in Hanebach 1423 II 5. MUW?

- de Nidernaltach 1436 II 4; lic. in decr. 1442 I 9. MUW 1, 1434 II R 5 (Sym. de Altach inferiori, profess[us] in Altach inferiori)

- prepositus Cibiniensis de Rozgon 1413 I 4. MUW 1, 1412 II H 52

Stephanus

- de Abara canonicus Agriensis 1428 II 4. MUW 1, 1425 I H 17 (Steph. de Albara)

- de Ehingen 1422 II 12 (als m. a.). MUW 1, 1417 I A 16 (Steph. Seussår de Ehingen)

- plebanus in Falkenstain 1413 I 11. MUW?

- Feyr de Clawsemburg 1431 II 4. MUW 1, 1417 II H 7? (Steph. de Clusenwar) oder 1426 I H 16? (Steph. Leonhardi de Chlüsbar)

- Grêtzzer, bacc. in decr. 1410 I 19. MUW 1, 1401 I A 28 (Steph. Grè-czer de Harenberg); Uiblein, AFA I, 563 (Steph. de Grăcz)

- Johannis de Grểuendorff 1422 I 7. MUW 1, 1423 I A 22? (Steph. Reysner de Greuendorff)

- Mardax 1429 I 8. MUW 1, 1419 II $^{2}$ A 10 (Steph. Mordax)

- Neumann de Freinstat 1430 II 9. MUW 1, 1427 II H 4 (Steph. Neyman de Libera Ciuitate)

- Phister de Steyer 1419 II 14. MUW 1, 1418 I A 18 (Steph. Phister de Steyrekk)

- Purgfinger plebanus in Purchusen 1402 I 7. MUW 1, 1402 I R 26 (Steph. Pürfinger)

- de Walthausen 1427 I 9. MUW 1, 1419 I A 33 (Steph. Leisentrit de Walthausen) 
- Wyrsing 1406 II 8. MUW 1, 1403 I _ - de Zelesna 1418 II 27. MUW 1 , A 21 (Steph. Wirsing) 1418 II $^{1}$ H 19 (Steph. de Zelezna)

- de Wusla 1430 I 3. MUW?

Theobaldus (Deobaldus, Tieboldus)

- Aichperger canonicus ecclesie Frisingensis 1429 II 14. MUW 1, 1422 I A 8 (Tybold. Aychperger de Mairing)

- de Frisinga 1429 II 9. MUW 1, 1426 I R 29 (Tybold. de Freysing)

- Tưrndl canonicus Patauiensis 1438 II 10. MUW 1, 1438 II R 47

Theodericus (Theodricus)

- de Brunswyg 1411 I 9 (als m. a.). MUW 1, 1411 I S 2 (Mag. Theodric. de Brunswik); Uiblein, AFA I, 564 (Theoderic. [de] Brunswik [Brunswig])

- de Fridland 1429 II 17. MUW 1, 1418 II $^{1}$ S 1 (Theodric. Fridlant de Prussia); Schrauf, AFM 1, 104 (Theodric. de Fridlant, bacc. med.)

- Lobing de Erdfordia 1410 I 8 (als m. a.). MUW 1, 1410 I R 58 (Mag. Tyderic. Lobingen de Ertfordia)

- de Staffel canonicus Maguntinus 1416 II 14. MUW 1, 1416 II R 19 (Theodor. de Staffel, can. maioris eccl. Maguntinensis)

Theodorus

- de Gich canonicus et archidyaconus Herbipolensis 1415 I 5. MUW 1, 1415 I R 58 (Theodoric. de Sig, archidiac. et can. eccl. Herbipolensis)

Tilmannus (Tylmannus)

- Helwich plebanus in Weydembach 1421 II 14. MUW 1, 1410 I H 11 (Tylm. Helbich de Porcia)

- Joel de Lyris [sic !] 1413 II 8. MUW 1, 1413 I R 98 (Tylm. Joyl de Lincz); Uiblein, AFA I, 565 (Tilm. de Lints [Linz]); Uiblein, Mittelalterliches Studium, $129 \mathrm{f}$.
Thomas

- de Achspach (O̊der) 1419 I 6 (als m. a.); bacc. in decr. 1422 I 2; lic. in decr. 1426 I 9; jur. Dekan 1426 II. MUW 1, 1404 II A 8 (Thom. de Aspach); Uiblein, AFA I, 565 (Thom. Öder de Axpach [Aspach])

- Angelpekh procurator ecclesie ad Sanctum Leonardum in Foresta 1433 I 3. MUW?

- Braxatoris de Amsteten 1414 I 11. MUW 1, 1414 I A 35

- de Coritnicza 1416 II 21. MUW 1, 1416 II H 39 (Thom. de Coritnicza de Cracouia)

- de Creyuelt plebanus in Pilichtorf 1432 I 14. MUW 1, 1428 II R 25 (Thom. de Chreuelt)

- Do̊rndel de Inferiori Altach 1413 II 17. MUW 1, 1413 II R 76 (Thom. Dornel de Altach inferiori)

- Furenríder de Amberga 1418 II 21. MUW?

- de Gelesdorff (Gelestorf) 1423 I 5; bacc. in decr. 1424 II 12; lic. in decr. 1437 II 22. MUW 1, 1417 I A 5 (Thom. de Gelesdorff)

- Hym de Brente canonicus ecclesie Vespermiensis 1430 I 13. MUW 1, 1428 I H 9 (Thom. de Brente)

- Liechtenuelder 1410 I 1. MUW?

- Lurczer 1413 II 2 (als m. a.). MUW 1, 1407 II A 30 (Thom. Lurczer de Sancto Ypolito); Uiblein, AFA I, 565 (Thom. Lurczer [Lurtzer, Lürtzer] de Sancto Yppolito)

- Mẻrher de Rastenueld 1419 II 8. MUW 1, 1415 I A 35

- Matzseảr decanus Maticensis $1411 \mathrm{I}$ 3. MUW 1, 1411 I R 10 
- de Norap canonicus ecclesie Jauriensis 1431 II 11. MUW 1, $1431 \mathrm{II} \mathrm{H}$ 29

- de Septemcastris 1410 I 2. MUW?

- de Septemcastris plebanus in Muschna 1411 II 16; bacc. in decr. 1413 I 29. MUW 1395 II H 7? (Thom. Helwich de Septemcastris) oder 1396 I H 4? (Thom. de Septemcastris) oder 1396 I H 27? (Thom. plebanus de Villa Petri in Septemcastris)

- Sybart de Rückpass 1437 II 9. MUW 1, 1430 II H 30

- Strohofer 1437 I 5; bacc. in decr. 1439 II 5 (als Dekan von Ardag- ger). MUW 1, 1417 II A 4? (Thom. Strahofer)

- de Tulna 1434 II 6 (als m. a.). MUW 1, 1421 II A 9 (Thom. Schawr de Tulna)

- de Vttendorff (Vttendorf) 1427 I 8 (als m. a.); bacc. in decr. 1431 I 11. MUW?

- de Zakan 1415 I 4 (als m. a.). MUW 1, 1411 I H 25 (Thom. Cosme de Sakan); Uiblein, AFA I, 566 (Thom. de Czakka[n]); Uiblein, AFTh 2, 704 (Thom. de Za[ch]kan); Schrauf, AFM 1, 108 (Mag. Thom. [de] Sacka, bacc. theol.); Schrauf, NH, 511 (Thom. de Zakan, mag. et proc. 1416 II)
Ulricus (Vdalricus)

- comes de Artemberg canonicus ecclesie Patauiensis et Ratisponensis 1418 II 9. MUW 1, 1418 II $^{1}$ R 14 (Vlr. de Artemberg, can. Patauiensis et Ratisponensis ecclesiarum, nec non rector parrochialis in Lincz)

- de Augusta Ordinis Sancti Benedicti 1429 II 13; bacc. in decr. 1433 I 14. MUW 1, 1428 II R 41 (Vlr. de Augusta)

- Kaltemprunner de Tegernsee $1441 \mathrm{I}$ 2. MUW 1, 1436 I R 66 (Vlr. Chaltenprunner de Tegernsee)

- de Kremsmünster 1438 I 7; bacc. in decr. 1441 I 13. MUW 1, 1435 II A 3

- Eysemman de Oீttinga 1424 I 8. MUW 1, 1421 I R 54

- Ërsinger 1438 I 8. MUW 1, 1431 I R 16 (Vlr. Åresinger, can. eccl. Frisingensis)

- de Frankfordia pedagogus ipsius1424 I 20 (siehe Adolffus de Eppenstain). MUW?

- Gewalt de Newmburga 1421 II 12. MUW?
- Håntinger plebanus in Bischofst(orf) 1440 I 4. MUW 1, 1440 I A 35

- de Haydreichstain magister chori ad Sanctum Stephanum Wiennensem 1431 II 1. MUW?

- Hawsecker 1409 I 5. MUW 1, 1385 II R 36 (Vlr. Husekker de Nůrenberga); Uiblein, AFA I, 566 (Ulr. Hausecker); Uiblein, Mittelalterliches Studium, 130

-Hawsner 1402 I 6. MUW 1, 1401 II R 46 (Vlr. Hausner)

- Hoffner 1432 I 4 (als b. a.). MUW 1, 1423 I R 47 (Vlr. Villicus de Kircham)

- Horant de Sweinfordia 1439 I 6 (als m. a.); bacc. in decr. 1439 I 12. MUW 1, 1438 II R 63 (Mag. Vdalr. de Sweinfordia)

- Horlactawer de Monaco plebanus in Holczhausen 1411 II 1. MUW 1, 1411 II R 1 (Vdalr. Harlatauer de Monaco, plebanus in Holczhausen)

- Lachmair 1431 I 3. MUW?

- Lager de Menning rector parochialis ecclesie in Ebelspach 1421 II 6. MUW 1, 1419 II $^{2}$ R 27 (Vlr. Langer de Memmingen) 
- Lang de Augusta 1433 I 5 (als m. a.). MUW 1, 1426 II R 13 (Vlr. Langenmantel de Augusta)

- de Mềnsee 1420 II 6. MUW 1, 1417 I R 59 (Vlr. de monasterio in Månsee)

- Nernshaymer de Haydenhaym 1415 II 6. MUW 1, 1415 II R 42 (Vlr. Nernshaymer de Haydenhaym, OSB)

- Nůsdorffer Patauiensis et Ratisponensis ecclesiarum canonicus 1436 II 2. MUW 1, 1432 I R 74 (Vlr. Nusdorffer prope Salczburgam); Uiblein, AFTh 2, 704 (Udalr. episc. Pataviensis)

- comes de O̊tingen cum domino Fridrico et Georio etc. 1429 II 1. MUW 1, 1429 II R 12

- de Orengew (Sunperger) 1430 II 3 (als m. a.); bacc. in decr. 1434 II 10; lic. in decr. 1438 I 15. MUW 1, 1425 II R 45 (Vlr. Sunneberger de Óringew); Uiblein, AFTh 2, 705 (Udalr. Sunnenberger, presul eccl. Gurcensis); Schrauf, AFM 2, 250 ([Ulr. III. Hinenberg de Sonnenberg] episc. Gurcensis)

- Prannt canonicus in Sliersee 1440 I 1. MUW 1, 1440 I R 55 (Vlr. Prannt, can. in Sliers)

- Reysperger canonicus Patauiensis, plebanus ad Sanctum Nicolaum prope Villacum 1419 I 13. MUW 1, 1413 II A 6 (Vlr. Reysperger de Valle Lauentina)

- Ruch 1429 I 17. MUW 1, 1429 I R 71 (Vlr. Rauch, can. eccl. Sancti Mauritii in Augusta)
- Stamhawser de Noua Ciuitate 1424 I 14. MUW 1, 1423 II R 72 (Vlr. Stainhauser de Noua Ciuitate)

- Waltheri de Streifdorf 1435 I 5. MUW 1, 1438 I R 115 (Vlr. Waltheri de Strewdorf)

- de Weyssemburga (Czand) 1415 II 7 (als m. a.); bacc. in decr. 1418 II 7; lic. in decr. 1427 II 10; jur. Dekan 1428 I (als lic. in decr.). MUW 1, 1411 II R 54 (Vlr. Czand de Weyzzenburga); Uiblein, AFA I, 567 (Ulr. [de] Weissenburga)

- Werder de Papenheim 1413 II 16. MUW 1, 1412 I R 6

- Werthart de Ratispona 1421 II 2. MUW 1, 1414 I R 95

- Czap de Bechthem 1429 I 13 (als m. a.). MUW 1, 1429 I R 65 (Vlr. Zapp, mag. in artibus)

Urbanus

- de Emerstorf 1416 II 24 (als m. a.). Wohl MUW 1, 1407 II A 23? (Vrb. de Emmerstorff) oder MUW 1, 1409 I A 23? (Vrb. de Melk); Uiblein, AFA I, 567 (Urb. de Em[m] erstorf [Mellico?])

- de Jawr (Sculteti) 1438 I 3; bacc. in decr. 1442 I 14. MUW 1, 1438 I H 43 (Vrb. Sculteti de Jawr)

- de Melk 1413 II 12 (als m. a.). MUW 1, 1409 I A 23? (Vrb. de Melk) oder MUW 1, 1407 II A 23? (Vrb. Vrbani de Emmerstorff); Uiblein, AFA I, 567f. (Urb. de Melck [Mellico]); Uiblein, AFTh 2, 706 (Urb. de Mellico [Medlico]); Uiblein, Mittelalterliches Studium, 130
Waltherus

- Scho̊rling canonicus Sancti Germani (als m. a.) 1412 I 18. MUW?

Wenceslaus (Wenczeslaus, Wencesslaus)
- de Brunna 1409 I 10. MUW 1, 1409 I H 5

- de Montibus Kutnis 1424 I 17 (als m. a.). MUW? 
- de Osterhofen 1418 II 11. MUW 1, 1397 II R 12 (Wenczesl. Johannis de Asternhouen); Uiblein, AFA I, 568 (Wenczesl. de Asterhofen)

- Sewberleich de Deschna 1416 II 13. MUW 1, 1416 II H 23 (Vencelaus Sewberleich de Deschna)

- Syndel (Sündel) canonicus ecclesie Sancti Petri in Brunna 1414 II 9; bacc. in decr. 1419 I 1 (de Brunna). MUW 1, 1414 II H 8 (Wencesl. Smudel de Bruna)

- de Sitauia 1422 I 8 (als m. a.). MUW 1, 1416 I H 36? (Wenslaus Igla de Zittauia)

- de Wessele plebanus in Wlocznow 1424 I 1. MUW 1, 1424 I H 7 (Wenczesl. de Wesselle, plebanus in Wlcznaw)

Wernherus (Berngerus)

- professus monasterii in Elbang 1430 I 8; bacc. in decr. 1433 II 9. MUW 1, 1430 I R 36 (Wernher. professus monasterii in Ellwang OSB)

Wigandus

- Menkler de Hohemberg 1423 I 3. MUW 1, 1417 I R 18

Wilhelmus

- Kircher (de Constancia) 1412 II 15 (als bacc. in decr.); jur. Dekan 1413 I (als m. a. und dr. decr.). MUW 1, 1411 II R 2 (Wilh. Kyricher de Constancia, bacc. in decr.); Uiblein, AFA I, 569 (Wilh. baccalarius); Uiblein, Mittelalterliches Studium, 130

- Kollner (Kolner) (de Danczk) 1414 I 21; bacc. in decr. 1416 II 39. MUW 1, 1414 I S 18 (Wilh. Kollner)

- Kraft de Vlma 1440 I 2 (als b. a.). MUW 1, 1437 II R 21

- Erhardi (Gerardi) (de Leydis) 1418 II 25 ; bacc. in decr. 1420 I 20; lic. in decr. 1421 I 7; dr. decr. 1421 I 26 (als scolasticus et can. Traioctensis). MUW 1, 1418 II $^{1}$ R 43 (Wilh. Gerhardi de Leydis)

- Grayspach de Monaco 1428 II 6 (als m. a.). MUW? Vielleicht identisch mit Uiblein, AFA I, 569? (Wilh. de Monaco)

- de Hürnhayn canonicus Augustensis 1417 I 18. MUW? (identisch mit Folgendem?)

- de Hurnhain canonicus ecclesie Augustensis 1413 I 6. MUW 1, 1412 II R 27 (Wilh. de Hurrenhaym, can. Augustensis) (identisch mit Vorigem?)

- Preyzyger canonicus Frizyngensis 1402 I 1. MUW 1, 1402 I R 30 (Wilh. Breysinger, can. Frisingensis)

- Pressater de Amberg 1413 I 18. MUW 1, 1413 I R 61 (Wilh. Pressatter, plebanus in Putreichsperig)

- Reàntel de Awsse 1415 I 18. MUW 1, 1411 I A 39 (Wilh. Reutel de Ausse)

- Span de Weyssenhorn 1421 I 4 (als m. a.). MUW 1, 1409 II R 36 (Wilh. Spann de Reissenhorn); Uiblein, AFA I, 569 (Wilh. Fabri de Weyßenhorn)

- Steger de Mittenbald 1429 II 26. MUW 1, 1424 I R 54 (Wilh. Steger de Mittenwald)

- de Weynsberg 1418 II 19 (als b. a.). MUW?

Wilibaldus

- Mayenschein 1437 I 8. MUW 1, 1436 II R 46 (Willib. Menschein de Eystauia)

Wolfgangus (Wolfgannus)

- de Admont 1417 I 10. MUW?

- Kerspek canonicus ad Sanctum Florianum 1418 I 12; bacc. in decr. 1421 II 16; lic. in decr. 1423 I 25. MUW 1, 1418 I A 17 (Wolfg. Cherspekch, can. monasterii Sancti Floriani)

- de Knüttefeld 1438 II 12 (als m. a.). MUW?

- Freythơfl de Gmunden 1438 I 10. MUW?

- de Greàtz 1438 I 4. MUW 1, 1430 I A 5? (Wolfg., filius Sutoris de Grecz) oder MUW 1, 1430 I A 14? (Wolfg., filius Coci de Grecz) 
- plebanus in Hartperg 1416 II 2. MUW?

- Payer (Payr) de Hederstorff 1421 I 9; bacc. in decr. 1430 I 26 (als plebanus in Czisterstorf). MUW 1, 1416 I A 12 (Wulgangus de Hedersdorf)

- Sachs (de Almekch prope Lambach) 1434 I 13; bacc. in decr. 1437 II 14. MUW 1, 1429 II A 8 (Wolfg. Sachs de Albenekk)

- de Tẻmsweg 1441 II 2. MUW 1, 1437 I A 50 (Wolfg. Mautter de
Temsweg)

- Temler de Stira 1430 II 24. MUW 1, 1426 I A 14 (Bolfgangus de Stira)

- de Weissenkirchen 1412 II 16 (als m. a.). MUW 1, 1406 II A 11 (Wolfgandus de Weißenkirchen); Uiblein, AFA I, 570 (Wolfg. de Weissenkirhen)

- Wurm (Wurm) de Freynstat 1414 I 6; bacc. in decr. 1437 II 15. MUW 1, 1414 I A 10 (Wolfg. Wurm de Libera Ciuitate)

$-\mathrm{C}, \mathrm{Cz}, \mathrm{Z}-$

Zegotha

- archidiaconus de Borswa et canonicus Agriensis 1412 II 21. MUW 1,
1412 II H 55 (Zegotha archidiac. de Worswa et can. eccl. kathedralis Agriensis) 



\section{Register der $\mathrm{Zu}$ - und Ortsnamen}

$-\mathrm{A}-$

Aalen (Kr. Ostalbkreis, RB Stuttgart, Baden-Württemberg, Dtld.) Ala Johannes Hieler 1429 II 23

Abara (ehem. Kom. Zemplén, Ungarn, slowak. Oborín, Slowakei)

Stephanus 1428 II 4

Absolonis

Petrus de Herdlof 1419 II 2

Achspach s. Aggsbach

Admont (GB Liezen, Stmk.) Admontensis

Wolfgangus 1417 I 10; Vitus 1420 II 12

Adorján (mehrfach in Ungarn und Slowa-

kei) Adorian

Nicolaus Clericus 1416 I 21

Åczmanspach s. Atzmannsbach

Ersinger

Vlricus 1438 I 8

Arkenstainer

Johannes 1424 I 5

Aggsbach (GB Spitz od. GB Melk, NÖ)

Achspach, Aspach

Thomas (O̊der) 1419 I 6; 1422 I 2;

1426 I 9; 1426 II

Agmanni

Petrus 1430 II 35

Agram (ungar. Zágráb, kroat. Zagreb, Kro-

atien) Zagrabia, Zagrabiensis

Johannes 1426 II 14, 1432 I 17;

Johannes (de) Bachenstain (Pa-

chenstain) 1430 I 28, 1431 II 18 ,

1432 I 16; Johannes de Bossna

1428 II 11, 1431 II 19; Nicolaus

1433 I 9; Petrus de Sulczpach 1426 I 1,1428 I 15

Agria, Agriensis s. Erlau

Aybling s. Bad Aibling

Aich (mehrfach in Bayern und Baden-

Württemberg, Dtld.) Eych

Johannes 1423 I 20, 1435 I, 1437 II
Aichelperger, Aychelperger

Johannes de Nouoforo 1429 II 5, 1432 I 18, 1435 I 10

Aichperger

Tieboldus 1429 II 14

Ala s. Aalen

Alba Jula, Albagywla s. Karlsburg

Alba Regalis s. Stublweißenburg

Alba Transsiluana s. Karlsburg

Albeins (italien. Albes, KG, Fraktion der Gem. Brixen, Südtirol, Italien)

Fredericus 1403 I 3

Alberti

Symon 1413 I 3

Albner

Virgilius de Salzburga 1420 II 20

Albrand

Johannes de Bruchsella 1412 I 13

Albrechtsberg (GB Ottenschlag od. GB

Melk, NÖ) Albrechtzperg

Marcus Enenkel 1416 I 17

Albrechtzperger

Georius 1421 I 8

Aldenpolan viell. s. Pölla und Pöllau

Aldersbach (Kr. Passau (Land), RB Niederbayern, Bayern, Dtld.) Olderspach Johannes 1402 I 4

Almår

Clemens de Salzpurga 1415 I 8

Almegg (Gem. Scharnstein, GB Lambach, $O O ̈)$ Almekch prope Lambach

Wolfgangus Sachs 1434 I 13, 1437 II 14

Alpirsbach (Kr. Freudenstadt, Baden-

Württemberg, Dtld.) Alperspacensis

Hugo de Leynsteten 1420 II 11

Altach Inferior, Altach s. Niederalteich

Altamuta s. Hohenmauth

Altbunzlau (tschech. Stará Boleslav, GB

Brandýs nad Labem, Böhmen, Tsche-

chien) Boleslauiensis, Bolislauiensis 
Matheus Slick (Slikch) 1428 II 3, 1430 II 31

Altenburg (GB Horn, NÖ) Altemburg Johannes 1411 I 17; Symon 1421 II 7

Altenmuldarff (viell. s. Mühldorf a. Inn) Althaymer Franciscus 1423 II 8

Alzen (ungar. Alcina, ehem. Kom. Szeben, rumän. Alțina, Siebenbürgen, Rumänien) Altzuna

Bartholomeus 1406 II 14

Amberg (Kr., RB Oberpfalz, Bayern, Dtld.) Amberga

Thomas Furenrider 1418 II 21; Wilhelmus Pressater 1413 I 18

Ambrosii

Johannes de Gvnczpurg 1423 I 19, 1426 I 11

Amelprecht

Johannes de Nicolspurga 1417 II 10

Amselèr

Johannes 1410 I 12

Amsteg

Petrus de Selgenstad 1415 II 4

Amstetten (GB, NÖ) Amsteten

Thomas Braxatoris 1414 I 11

Anasum s. Enns

Andree

Gregorius de Molhausen 1442 I 7;

Matheus de Lyppa 1441 I 7; Nicolaus de Strềlen 1414 II 14

Angeli

Jacobus de Vlma 1417 II 12

Angelpekh

Thomas 1433 I 3

Ansbach (Kr., RB Mittelfranken, Bayern,

Dtld.) Anspach, Anolspach, Onal-

bach

Johannes Newsteter 1416 II 27;

Petrus Hoszil 1417 I 2, Jacobus

Hofeman 1405 II 1

Apoldia Inferior s. Klein-Apolden

Ardagger (GB Amstetten, NÖ) Ardacensis

Eglolfus 1406 II 6; Heinricus Fleckl 1438 II 14; Thomas (Strohofer) 1437 I 5; bacc. in decr. 1439 II 5
Argentina, Argentinensis s. Straßburg

Argyas (ehem. Kom. Kolozs, rumän.

Arghişu, Siebenbürgen, Rumänien)

Ardisch

Michael Ekhart 1434 I 10

Arnoldi

Conradus 1430 I 20

Arnolt

Cristofferus de Tullaco 1429 II 12; Johannes de Tullaco 1429 II 11

Arnsdorf, Mitter-od. Ober- (GB Spitz,

NÖ) oder (Arndorf, GB Pöggstall, NÖ)

Arnsdorf

Mag. Petrus 1409 II 7

Artenberg, Artemberg s. Ortenburg

Aspach s. Aggsbach

Asparn (a. d. Zaya, GB Mistelbach, NÖ

oder Gem. Langenrohr, GB Tulln,

NÖ) od. Aspern a. d. Donau, 22. Bez.,

Wien) Asparn, Asparn Mayor

Erhardus 1441 I 1; Nicolaus 1420 I 10; Symon 1422 I 19, 1440 I 9, 1440 II 10

Aspekch

Gundakerus 1410 II 3

Astauia s. Eichstätt

Astershofen s. Osterhofen

Atyser

Nicolaus de Villa Las 1415 II 3

Atzmannsbach (Gem. Leiben, GB Melk,

$N O ̈$ ?) Aczmanspach, Åczmanspach

Michael (Michel), 1402 I 8, 1407, 1408 II, 1410 II, 1414 II

Au oder Aub (mehrfach in Süddeutsch-

land) Aw

Andreas Putnyt 1418 I 11; Johannes Holczman 1416 II 23

Aug-Radisch (GB Feldbach, Stmk.) Rèdisch

Michel 1424 II 9

Augsburg (kreisfr. Stadt, RB Schwaben, Bayern, Dtld.) Augusta, Augustensis Gabriel 1427 I 2; Gabriel Lang 1435 I 6; Gotfridus Harscher 1411 II 19; Heinricus Burggrauius 1423 I 9; Johannes Pencznalber 1438 II 8; Mag. Petrus 
1409 I 8; Rudolfus Westersteter 1412 I 19; Siluester (Flieger) 1411 II 12; bacc. in decr. 1415 II 12?; Vdalricus Ordinis Sancti Benedicti 1429 II 13; bacc. in decr. 1433 I 14; Mag. Vdalricus Lang 1433 I 5; Wilhelmus de Hurnhain 1413 I 6; Wilhelmus Augustini de Hůrnhayn 1417 I 18

Johannes de Ratispona 1414 II 13 Aussee (entweder Altaussee, GB Bad Aussee,
Stmk. oder Bad Aussee, Stmk.) Awsse

Wilhelmus Rẽntel 1415 I 18 Außernzell (Kr. Deggendorf, RB Niederbayern, Bayern, Dtld.) Aussernczel Johannes Mulhaimer 1434 I 6 Aw s. $A u$

Awer

Johannes 1416 I 24

Awsse s. Aussee

Aczmanspach s. Atzmannsbach

Aczo

Sigismundus 1433 II 6
Bachenstain, Pachenstain

Johannes 1430 I 28, 1431 II 18, 1432 I 16

Bacia, Baciensis s. Waitzen

Back

Martinus de Gamundia 1410 II 4 Bad Aibling (Kr. Rosenheim (Land), RB Oberbayern, Bayern, Dtld.) Aybling Sigismundus Walich 1426 II 17

Bad Canstatt (Stadtteil von Stuttgart, Baden-Württemberg, Dtld.) Chonstatt Luthardus 1429 II 25

Bad Schussenried (Kr. Biberach, RB Tübingen, Baden-Württemberg, Dtld.) Schussenried Petrus Fuchs 1431 II 10, 1433 II 11

Bad Waldsee (Kr. Ravensburg, RB Tübingen, Baden-Württemberg, Dtld.)

Walsee

Johannes Sigel 1412 II 4

Baden (GB, NÖ oder Kanton Aargau, Schweiz)

Jacobus 1414 II 17

Beartel s. Pértl

Bayreuth (Kr., RB Oberfranken, Bayern, Dtld.) Perawt Jacobus Blassemberger 1419 I 15 Bamberg (kreisfr. Stadt, RB Oberfranken, Bayern, Dtld.) Bamberga, Bambergensis

Fridericus Schenkch de Lymburg
1411 II 8; Georgius Hohenloch 1435 I 1; Georius de Schawmberg 1415 II 9; Henricus 1415 II 10

Bano s. Wanow

Baradinum, Baradiensis s. Großwardein

Barenghůt s. Brekolten

Barna s. Bernau

Bartholomei

Petrus de Görlitz 1437 I 10

Basel (Schweiz) Basilea, Basiliensis

Bernhardus de Raczenhausen 1412 I

22; Johannes Czwinger 1424 I

13; Rudolfus 1418 I 16

Bátmonostor (Benediktinerabtei, Kom.

Bács-Bodrog, Bez. Bajai, Ungarn)

Bata, Batha

Paulus (de Sana) 1414 I 16, 1417 II 3

Bátor (mehrfach in Ungarn) Bathor

Benedictus 1418 II 15

Battaw s. Rothau

Baumburg (Gem. Altenmarkt a. d. Alz, Kr.

Traunstein, RB Oberbayern, Bayern,

Dtld.) Pawmburg, Påmburg, Pawburgensis

Caspar Ebenhawser 1428 II 10, 1431 II 23; Jeronimus 1416 II 18

Baustetter

Bernhardus de Maisemburg 1438 II 16 
Bautzen (wohl Kr., RB Dresden, Sachsen, Dtld.) Budesheim Heinricus Copericz 1417 I 20

Bawdewek Jacobus 1416 II 26

Bechlingen (Stadt Aßlar, Kr. Lahn-DillKreis, RB Gießen, Hessen, Dtld. oder Gem. Ruppichteroth, Kr. Rhein-SiegKreis, RB Köln, Nordrhein-Westfalen, Dtld. oder Stadt Tettnang, Kr. Bodenseekreis, RB Tübingen, Baden-Württemberg, Dtld.) Lechling Michael 1414 I 4

Bechtheim (Kr. Alzey-Worms, RB Rheinhessen-Pfalz, Rheinland Pfalz, Dtld.) Bechthem Johannes Czap 1429 I 12; Vlricus Czap 1429 I 13

Beckensloer

Jeronimus de Wratislauia 1439 I 5

Bellazon

Rudolfus 1411 II 7, 1414 I 29

Bensheim (wahrscheinl. Kr. Bergstraße, RB Darmstadt, Hessen, Dtld.) Pemeshaim Conradus 1430 I 18, Petrus 1430 I 19

Berching (Kr. Neumarkt i. d. Opf., RB Oberpfalz, Bayern, Dtld.) Perching Johannes Gartner 1419 I 14

Berchtoltstorf s. Perchtoldsdorf

Berlichingen (Gem. Schöntal, Kr. Hohenlohekreis, Baden-Württemberg, Deutschland)

Beringerus 1436 II 8, 1436 II 11

Bernau bei Berlin (Kr. Barnim, Brandenburg, Dtld.) Barna, Warnaw Petrus (de Prusia) 1414 I 19, 1416 I 31, 1416 II 36, 1416 II 40

Bernicz s. Pernicz

Bernstein (Mark Brandenburg, poln. Petczyce, Polen) Bernsteyn, Pernstaìn Hinricus 1402 I 2, 1406 Februar 4, 1405 II

Berwardi Johannes 1401 II

Beschorn Mathias de Noua Ciuitate 1421 II 8
Bethneri

Nicolaus 1433 II 5

Biberach (Baden-Württemberg, Dtld. mehrfach) Bybracum

Johannes 1418 II 3

Biburg (Bayern, Dtld. mehrfach) Piburga Caspar (Westerndorffer de Lantzhout) 1433 II 16, 1436 I 6

Byla s. Weil

Birchachhusen, Pirchachhausen (unbestimmt, $A$ )

Fredericus Sartoris alias Maler 1429 II 8, 1435 I 15, 1435 II 14

Birthälm, Birthelm (ungar. Berethalom, ehem. Kom. Nagy-Küküllo, rumän. Biertan, Siebenbürgen, Rumänien)

Wirthelm

Sigismundus (de Septemcastris) 1409 II 10, 1412 II 14

Bischofdorf (Bez. Cilli, slowen. Škofja Vas (priu Celju), Gem. Cilli/Celje, Slowenien) Bischofst(orf)

Vlricus Hăntinger 1440 I 4

Blabeuren s. Blaubeuren

Bladeck, Bladek

Conradus de Wienna 1420 I 3, 1424 II 10, 1426 I 10, 1427 I

Bladen (poln. Wtodzienin, Schlesien, Polen)

Johannes (Fůlstein) 1436 II 1, 1441 II 6

Blassemberger

Jacobus de Perawt 1419 I 15

Blaubeuren (Kr. Alb-Donau-Kreis, RB Tübingen, Baden-Württemberg, Dtld.)

Blabeuren

Heinricus Megenhart 1424 I 4

Bochdaschin (tschech. Bohdašin, GB Náchod, Böhmen, Tschechien oder Bohdašin, GB Nové Mešto nad Metuji, Böhmen, Tschechien oder Podieschin, tschech. Poděšin, Böhmen, Tschechien) Podatzschin Johannes Koslick 1430 I 1

Bodo

Nicolaus 1435 II 4 
Bodon oder Bodony (Ungarn mehrfach) Boden

Michael 1430 II 5

Boldmensis (unbestimmt, $S$ ) Johannes Ludberti 1415 II 8

Boleslauiensis, Boleislouiensis s. Altbunzlau

Boll (Baden-Württemberg, Dtld. mehrfach)

Johannes de Stukardia 1418 II 13

Borystawice (viell. Ort östl. von Konin, Polen) Borzislauicze

Albertus 1414 I 10

Borsova (wohl Kom. Bereg, Ungarn)

Borswa

Zegotha 1412 II 21

Boskowitz (viell. tschech. Boskovice, Mähren, Tschechien) Possilwicz

Johannes Jewkewicz 1422 II 5

Bosnonensis s. Preßburg

Bosnien (serbokroat. Bosna, Einzugsgebiet der Save innerhalb der Republik Bosnien-Herzegowina) Bossna, Bozna Johannes 1428 II 11, 1431 II 19

Bradislauia, Bratislauia, Bratislauiensis s. Breslau

Braunau am Inn (GB, OÖ) Prawnaw Georius Melchaymer 1408 II 1

Braunsberg (ehem. RGB Königsberg, Ostpreußen, poln. Braniewo, Polen) Brounsperg, Prawnsperg Johannes Turinge 1413 I 24; Nicolaus Martini de Prussia 1436 I 3

Braunschweig (kreisfr. Stadt, Niedersachsen, Dtld.) Brunswyg

Theodricus 1411 I 9

Braxatoris

Thomas de Amsteten 1414 I 11

Bregensis s. Brieg

Brekolten (ungar. Báránykút, ehem. Kom. Nagy-Külküllö, Bez. Nagysinki, rumän. Bărcut, Siebenbürgen, Rumänien) Barenghůt Georgius 1437 II 7

Brennberg (wahrscheinl. Kr. Regensburg (Land), RB Oberpfalz, Bayern, Dtld.) Preinberg
Kaspar 1440 II 9

Brente (unbestimmt, $H$ )

Thomas Hym 1430 I 13

Breslau (Niederschlesien, poln. Wroctaw,

Polen) Bradislauia, Bratislauia, Bratis-

lauiensis, Vratislauiensis, Wratislauia,

Wratislauiensis

Andreas Michaelis 1425 II 5; Cristoforus Piderman 1437 I 2; Vincencius de Jordansmül 1418 I 4; Gabriel 1442 I 1; Gregorius de Cosula 1416 I 12; Jeronimus Beckensloer 1439 I 5; Johannes Briger 1433 I 2; Johannes Fleckel 1438 II 15; Johannes Fülstein de Bladen 1436 II 1, 1441 II 6; Johannes Füssel 1411 II 9, 1416 I 28; 1420 I 16, 1420 II 24; Johannes Vey 1421 I 24; Johannes Gnechowicz 1411 I 2; Johannes Godke 1418 I 14; Johannes [de] Grecz Regine 1427 II 4; Johannes Yencz 1419 I 4; Johannes Rademan de Lukau 1430 I 5; Johannes Lamberg 1420 II 4; Johannes Scholym 1415 I 11; Johannes Sponsberg 1425 II 2; Johannes Sweydnitz 1410 I 13; Laurencius Kasla 1415 I 6; Martinus in Habelswerd 1420 II 13; Martinus Petirbicz 1412 I 10; Michael Stankonis 1426 I 7; Nicolaus Bethneri 1433 II 5; Nicolaus Vogel 1412 I 3; Nicolaus Gramus 1422 II 1; Nicolaus Mathie Goldperg 1410 I 14, 1416 II 38; Nicolaus amicus decani Patauiensis 1413 I 33; Nicolaus Reychel de Wratislauia 1433 II 1; Nicolaus Stok de Maiori Glogouia 1413 I 17, 1421 I 20, 1422 I 25; 1422 II; Nicolaus Wenke 1422 II 2, 1423 II 9; Petrus Teschner 1411 I 13; Procopius Stampen 1421 II 19

- Diözese Johannes de Goltperg 1435 II 9, 
1437 II 20; Johannes Jonsdorf 1412 I 25; Johannes Sutoris de Freynstat 1437 II 2

Brezewicz (unbestimmt)

Caspar Sepusiensis 1426 II 11

Brieg (Niederschlesien, poln. Brzeg, Polen)

Bregensis

Nicolaus Zedlicz 1418 I 17

Briger

Johannes 1433 I 2

Bringk in Ergaw s. Brugg

Brisach

Johannes de Constancia 1415 I 19

Briswicz (unbestimmt)

Georius Petri 1425 I 2

Brixen (italien. Bressanone, Südtirol, Ita-

lien) Brixina, Brixna, Brixenensis

Cristannus de Friberg 1440 II 4; Johannes 1427 I 11; Johannes

Schenkchenberger 1412 I 15;

Nicolaus Perold 1403 I 2, 1411 II

18?; [Nicolaus Swarat] 1420 I

21; Philippus Swarat 1433 I 7

1436 II 18,1440 II 13

Brounsperg s. Braunsberg

Brucenus Johannes 1415 II 5

Bruchenstain (unbestimmt)

Johannes 1427 I 3

Bruchsal (Kr. Karlsruhe [Land], RB Karlsruhe, Baden-Württemberg, Dtld.)

Bruchsella, Bruxella, Pruszella Johannes Albrand 1412 I 13; Johannes Traboldi 1411 II 3; Petrus Crombach 1412 I 8

Bruck an der Mur (GB, Stmk.) Prugk

Erhardus Kornmecz 1440 I 3

Brünn (tschech. Brno, Mähren, Tschechien)

Brunna, Brünna, Brunnensis

Cristannus de Brunna 1409 I 4; Johannes Hers (Hêz, Has) 1429 I 1, 1432 I 20, 1438 I 14; Johan- nes Nicolai 1412 II 7; Johannes Polzmacher (Poltzmacher, Polczmacher) 1428 I 4, 1430 I 34, 1432 II 8, 1434 II 13, 1436 I, 1439 I, 1441 I; Maternus Tewfel 1440 II 2; Wencesslaus 1409 I 10; Wenczeslaus Syndel (Sündel) 1414 II 9, 1419 I 1

Brugg (Kant. Aargau, Schweiz) Bringk in Ergaw

Eglolfus Etterli 1412 II 10

Brunn (NÖ mehrfach) Prün

Lucas Slitt 1426 II 12

Brunswyg s. Braunschweig

Bruxella s. Bruchsal

Budapest (Hauptstadt von Ungarn) Buda

Briccius 1417 I 3

Budesheim s. Bautzen

Buerser

Hermannus de Veltkilch 1409 I 3

Burggrauius

Heinricus 1423 I 9

Burghausen Kr. Altötting (Kr. Altötting, RB Oberbayern, Bayern, Dtld.)

Burghusen, Purkhawsen, Purchhawsen, Purkhusa

Johannes Lehener 1423 I 11; Nicolaus Erlacher 1431 II 5, 1435 I 8; Stephanus Purgfinger 1402 I 7

Burmeyster

Andreas de Gribiswaldis 1416 I 13

Burzenland (ungar. Barcaság, rumän. Țara Bîrsei, Bez. Kronstadt, Siebenbürgen,

Rumänien) Wurcia

Cristannus de Hiltenstorff 1429 II 20

Bušince (Slowakei, ungar. Bussa, ehem. Kom. Nógrád) Busa, Wusla Antonius 431 II 3; Stephanus 1430 I 3

Butzbach (Kr. Wetteraukreis, RB Darmstadt, Hessen, Dtld.) Butczpach Johannes 1410 I 17 
Kärnten (österr. Bundesland, Grenzgebiete heute bei Italien und Slowenien) Carinthia, Karinthia

Erasmus Teisser 1436 II 6; Johannes Kèser 1440 II 3

Kèser

Johannes 1440 II 3

Kalbskoph

Caspar 1425 I 1

Caldarium s. Kaltern an der Weinstraße

Kalman

Nicolaus de Hoczemplocz 1423 I 4

Kaltemprunner

Vlricus de Tegernsee 1441 I 2

Kaltenbrunn (tschech. Kaproun, GB NeuBistritz, Böhmen, Tschechien oder Studánky, GB Kdynè, Böhmen, Tschechien) Nicolaus Johannis 1418 I 6

Kaltern an der Weinstraße (italien. Caldaro sulla Strada del Vino, Südtirol, Italien) Caldarium

Petrus de Lynss 1416 I 11

Campa s.Zánka

Campidena, Campidona s. Kempten

Chanadiensis s. Csanád

Kanth (poln. Katy Wroctawskie, Niederschlesien, Polen) Kant

Nicolaus Paskowicz 1426 II 5

Kappel (Baden-Württemberg, Bayern, Hessen, Rheinland-Pfalz mehrfach)

Cappel, Cappell

Hartungus de Cappel 1432 I 7, 1433 I

Carinthia, Karinthia s. Kärnten

Karlowitz (entweder ungar. Karlóca-Srijemski Karlovci, ehem. Kom. Szerém, serb. Sremski Karlovci, Serbien oder Stadtteil von Breslau, poln. Wroctaw, Niederschlesien, Polen) Carlowicz Heinricus Roll 1414 II 12

Karlsburg (früher Ungar.-Weißenburg, ungar. Gyulaféhérvár, ehem. Kom. AlsóFehér, rumän. Alba-Julia, Siebenbürgen, Rumänien) Alba Jula, Albagywla, Alba Transsiluana
Johannes 1437 I 4, 1438 II 22;

Petrus 1420 II 14, 1423 I 28, 1425 II 6

Karpfen (ungar. Korpona, ehem. Kom.

Hont, slowak. Krupina, Slowakei)

Corpona

Laurencius Stephani 1420 I 7

Kasla

Laurencius de Wratislauia 1415 I 6

Chasma, Chasmensis, Casmensis $s$. Čazma

Cassela s. Kosel

Čazma (wohl ehem. Kom. Belovár-Körös, heute Kroatien) Chasma, Chasmensis, Casmensis

Johannes de Bozna (Bossna) 1428 II 11, 1431 II 19; Nicolaus 1416 II 19, 1420 I 17, 1422 I 24

Kecskemét (Verw. Sitz des Kom. BácsKiskun, Ungarn) Keczkemeth Albertus 1426 II 13

Kellner

Leonhardus de Wirgil 1435 I 4

Kemerêr

Erasmus 1412 I 12

Kempten (Allgäu) (kreisfr. Stadt, RB

Schwaben, Bayern, Dtld.) Campidena, Campidona

Conradus de Campidena 1422 I 22; Jodocus Maursteter 1438 II 9; Petrus Kieler 1429 II 21

Cerdonis

Petrus 1420 II 16

Kerkingen (Stadtteil von Bopfingen, Kr.

Ostalbkreis, RB Stuttgart, Baden-

Württemberg, Dtld.) Korkyngen

Oswaldus 1402 I 11

Kern

Johannes de Kůlsheim 1435 II 6

Kerot s. Csejd

Albertus 1419 I 10

Kerspek

Wolfgangus 1418 I 12, 1421 II 16, 1423 I 25

Kyczka s. Kitschka 
Kieler

Petrus 1429 II 21

Kykuw (unbestimmt, $H$ )

Demetrius Czuporis 1429 I 6

Kynsperger

Nicolaus de Chrewsen 1430 II 14

Kirchain s. Kirchhaim

Kirchdorff

Laurencius de Rota 1418 I 18

Kirchen

Johannes iunior 1414 II 7

Kircher

Wilhelmus (de Constancia) 1412 II 15, 1413 I

Kirchhaim oder Kirchheim oder Kirchham oder Kirchen (mehrfach in Dtld.)

Kirchain, Kirchhain

Johannes Hågg 1424 I 3; Petrus Sartoris 1437 I 11

Kirchhof

Erasmus de Goth 1442 I 5

Kitschka, Kitzka, Kyczka

Nicolaus 1411 II 17, 1413 I 30, 1419 II 15

Kitzbühel $(G B, T)$ Kitzpuhel, Kitzpůhel

Heinricus 1407 II, 1410 I

Kitzingen (Kr., RB Unterfranken, Bayern, Dtld.) Chiczing, Kyczing

Conradus Speng 1437 I 12; Johannes 1422 I 17

Klausenburg (ungar. Kolozsvár, rumän. Cluj, Siebenbürgen, Rumänien)

Chlausenburga, Clausenburk, Clawsemburg

Jacobus de Clausenburk 1428 I 8, 1430 II 29; Nicolaus Kôschel 1414 II 3; Stephanus Feyr 1431 II 4

Klein-Apolden (ungar. Kisapold, ehem. Kom. Szeben, Bez. Szerdahelyi, rumän. Poldá mika, Rumänien) Apoldia Inferior Paulus 1435 II 5

Clementis

Nicolaus 1418 II 28

Kleve Kr. Kleve, (Kr. Kleve, RB Düsseldorf, Nordrhein-Westfalen, Dtld.) Cliuis Johannes Preym 1416 I 7
Klingenstainer

Michael 1437 I 18

Cliuis s. Kleve

Clodebogk (Klodebokch, Klodebokch)

Heinricus 1420 I 12, 1423 II 11, 1430 I 27

Klodechk

Henricus 1427 II 3

Chlosner

Andreas de Geren 1415 II 1; Johannes 1419 I 7

Klosterneuburg (GB, NÖ) Newmburga

Claustralis, Newmburgensis, Newnburga, Newnburgensis, Neuburgensis, Neunburgensis

Andreas 1432 I 5; Bernardus 1432 I

6; Cholomanus (Knapp de Hyppleins) 1422 I 1, 1425 II 7 , 1428 II 12, 1430 II 33; Erhardus Herrant 1422 I 20, 1425 II 3, 1430 I 30, 1432 I 15, 1430 II, 1433 II; Georius 1406 II 3; Johannes de Perchtoltzdorff 1406 II 2, 1421 II 1, 1422 I

Cluting (Gloting)

Nicolaus de Traiecto 1419 I 22, 1423 I 31

Knapp

Cholomanus 1422 I 1, 1425 II 7, 1428 II 12, 1430 II 33

Knittelfeld (GB, Stmk.) Knüttefeld

Wolfgangus 1438 II 12

Knot

Cyriacus 1423 I 18

Coburg (kreisfr. Stadt, RB Oberfranken, Bayern, Deutschland) Choburg Heinricus 1422 I 16

Cochem (Kr. Cochem-Zell, RB Koblenz, Rheinland-Pfalz, Dtld.) Chochaim Johannes 1434 I 3

Köllner, Kolner Wilhelmus de Danczk 1414 I 21, 1416 II 39

Köln (kreisfr. Stadt, RB, Nordrhein-Westfalen, Dtld.) Colonensis Adolffus de Eppenstain 1424 I 19 
Königgrätz (tschech. Hradec Králové, Böhmen, Tschechien) Grecz Regine Johannes 1427 II 4

Königsberg (Ostpreußen, russ. Kaliningrad, Rußland) Kònigsperg, Künigsperg, Kunigsberg Jodocus Quedenow 1416 I 19; Petrus Hecht 1413 I 1, 1416 I 29; Nicolaus Satler 1441 I 6

Königshofen (Stadtteil von Lauda-Königshofen, Kr. Main-Tauberkreis, RB Stuttgarrt, Baden-Württemberg, Dtld.) Kunigshofen Johannes Lapicide 1437 II 5

Königstetten (GB Tulln, NÖ) Kunigsteten Caspar Kalbskoph 1425 I 1

Kòschel

Nicolaus de Clawsemburg 1414 II 3

Coloniensis s. Köln

Conradi

Pangracius de Sweinbart 1430 I 6

Konstanz (Kr., RB Freiburg i. Br., BadenWürttemberg, Dtld.) Constancia Fredericus Tifer 1420 II 2; Gebso $\mathrm{H}^{\mathrm{a}}$ ewler 1426 I 2; Johannes Brisach 1415 I 19; Wilhelmus Kircher 1412 II 15, 1413 I

Chonstatt s. Bad Canstatt

Copericz

Heinricus de Budesheim 1417 I 20

Cordobok

Mathias de Gora 1411 I 4

Coritnicza (unbestimmt, $H$ )

Thomas 1416 II 21

Korkyngen s. Kerkingen

Kornburg (kreisfr. Stadt Nürnberg, RB Mittelfranken, Bayern, Dtld.)

Fridericus Flokch 1440 II 7

Kornmecz

Erhardus de Prugk 1440 I 3

Corona s. Kronstadt

Corpona s. Karpfen

Kosel (Stadtteil von Breslau oder Cosel poln. Koźle, Oberschlesien, Polen) Cassela, Cosula

Gregorius 1416 I 12; Hermannus 1416 II 3
Koslick

Johannes de Podatzschin 1430 I 1

Chotter

Petrus de Patauia 1416 II 12

Krèt

Nicolaus 1410 I 11

Kraft (Krafft)

Fridricus 1406 II 15, 1417 II 6, 1417 II 7, 1418 I 3, 1418 I; Wilhelmus de Vlma 1440 I 2

Krakau (poln. Kraków, Galizien, Polen)

Cracouiensis

Albertus de Borzislauicze 1414 I 10; Jeroslaus de Sluskow 1426 II 1; Paulus de Gleybicz 1436 I 1, 1438 II 20, 1442 I 8

Chramer

Petrus 1431 I 2

Chranach s. Kronach

Kranpergher

Hinricus 1403 I 1

Krefeld (kreisfr. Stadt), RB Düsseldorf, Nordrhein-Westfalen, Dtld.) Creyuelt

Thomas 1432 I 14

Kreig (Kraig) (Kärntner Herrengeschlecht)

Kreyg, Chreig

Conradus 1431 II 15, 1432 I 1

Kremnitz (ungar. Körmöcbánya, ehem.

Kom. Bars, slowak. Kremnica, Slowa-

kei) Cremniczia

Johannes Telkener 1432 II 5

Krems (GB, NÖ) Chremsa

Johannes Ebner 1420 I 8

Kremsier (tschech. Kroméríž, Mähren,

Tschechien) Kremsirensis, Chremsi-

rensis

Heinricus Roll 1414 II 12; Johannes [de] Grecz Regine 1427 II 4

Kremsmünster (OÖ, Benediktinerstift)

Kremsmünster

Vlricus 1438 I 7, 1441 I 13

Kren

Bartholomeus de Erdpurg 1410 I 5

Creußen (Kr. Bayreuth (Land), RB Oberfranken, Bayern, Dtld.) Chrewsen

Nicolaus Kynsperger 1430 II 14 
Kreuzstetten (mehrfach in Ö) Grewibtzensteten

Martinus Czeller 1423 I 12, 1431 I 14

Cristanni

Nicolaus de Reddin 1420 I 2

Cristoferi

Johannes de Winczig 1413 II 15

Crombach

Petrus de Pruszella 1412 I 8

Kronach (Kr., RB Oberfranken, Bayern,

Dtld.) Chranach, Tranbach

Conradus Schürg 1413 I 23; Martinus 1412 II 19

Kronstadt (ungar. Brassó, rumän. Braşov,

Siebenbürgen, Rumänien) Corona

Georius (de Septemcastris) 1433 II

18, 1436 II 16, 1440 I 11; Jo-

hannes 1414 I 8; Johannes Gůt

1437 II 8, 1440 I 8; Johannes

(Råbel) 1424 II 7, 1427 II 13;

Johannes Wilhelmi 1424 II 2;

Laurencius 1429 I 10, 1430 II 32;

Seruacius 1426 II 7

Kronberg (Ministerialengeschlecht im Tau-

nus, Dtld.) Cronemberg

Franco 1414 I 3; Phylippus 1414 I 2

Crossen (Oder) (Mark Brandenburg, poln.

Krosno Odrzańskie, Polen) Crossna,

Chrussen

Johannes (Toliatoris) 1429 I 3, 1430 II 26

Krotendarffer

Nicolaus 1410 I 6

Csanád (Ungarn und Rumänien mehr-

fach) Chanadiensis

Blasius 1419 I 9; Ladislaus de Marcellis 1419 I 8

Császár (Kom. Komárom, Ungarn)

Czschach

Ladislaus 1431 I 13

Csejd (wohl ehem. Kom. Maros-Torda,

Ungarn, rumän. Cotuš, Rumänien)

Czerod

Demetrius 1412 II 17

Csütörtökhely (ehem. Kom. Szepes, slowak. Spišsy Štvrtok, Slowakei) Villa Las
Nicolaus Atyser 1415 II 3

Chuefstain s. Kufstein

Külsheim (wohl Külsheim Main-Tauber-

Kreis, Kr. Main-Tauber-Kreis, RB

Stuttgart, Baden-Württemberg, Dtld.)

Külsheim

Conradus Schenkch 1418 II 17; Johannes Kern 1435 II 6

Künigsperg s. Königsberg

Künlein, Kuenlein

Conradus de Dyppurg 1429 II 4, 1431 II 24

Kürsner, Kưrsnêr

Andreas de Villseck 1439 I 8, 1441 II 8

Kürtös (entweder ehem. Kom. Arad, rumän

Sânpaul, Rumänien oder Garamkür-

tös, ehem. Kom. Bars, slowak. Trubin,

Slowakei oder Kiskürtös, ehem. Kom.

Nógrád, slowak. Maly Krtiss, Slowakei

oder Nagy Kürtös, ehem. Kom. Nógrád, slowak. Velký Krtís, Slowakei) Gưr-

tesch

Johannes 1436 I 4

Kufstein $(G B, T)$ Kufstain, Chuefstain,

Chweffstain

Cristannus Mulpacher 1426 I 6; Jacobus 1426 II 9, 1426 II 22

Kugelreawter

Wernhardus 1418 II 29

Kulled

Matheus 1426 II 18

Kunigsberg s. Königsberg

Nicolaus Satler 1441 I 6

Kunigshofen s. Königshofen

Kunigsteten s. Königstetten

Chur (Kant. Graubünden, Schweiz) Curcensis, Curiensis

Rudolfus Bellazon 1411 II 7, 1414 I 29

Kuttenberg (tschech. Kutná Hora, Böhmen,

Tschechien) Montes Kutnae, Montes

Chutnae

Nicolaus Reiberstorffer 1412 II 9;

Wenczeslaus 1424 I 17

Chweffstain s. Kufstein 
Dachsawer

Cristoforus 1434 II 3

Dänemark Dacia

Nicolaus Johannis 1420 II 10

Dagersheim (Stadtteil von Böblingen, Kr., RB Stuttgart, Baden-Württemberg, Dtld.) Tagersheim, Tagersheym, Tagershaim, Tagerhaim

Heinricus Rawch 1417 I 17, 1421 I 16; Johannes 1411 I 11, 1413 I 12, 1414 I 30

Danzig (poln. Gdańsk, Polen) Danczk Johannes Fuchs 1425 I 4; Johannes Gödke 1412 I 4; Luwertus Lankow 1424 I 15; Wilhelmus Kolner 1414 I 21, 1416 II 39

Deckinger (Dekchinger, Dekinger)

Petrus 1402 I 12, 1412 II, 1418 II, 1419 II 18

Deys

Hermannus 1417 II 14; Johannes 1418 I 20

Deringaw

Heinricus 1413 I 10

Deschna (entweder tschech. Dešná-

Roubanina, GB Jevičko, Mähren, Tschechien oder tschech. Deštná, GB

Kamenice nad Lipou, Böhmen, Tschechien oder Gem. Brodek u Konice, GB Konice, Mähren, Tschechien oder tschech. Desná, GB Leitomischl, Böhmen, Tschechien oder tschech. Dešná, GB Vizovice, Mähren, Tschechien) Wenczeslaus Sewberleich 1416 II 13 Dieburg (Kr. Darmstadt-Dieburg, RB Darmstadt, Hessen, Dtld.) Dyppurg,
Dyeppurg

Conradus Künlein (Kuenlein) 1429 II 4, 1431 II 24

Diessenhofen (Kant. Thurgau, Schweiz)

Dyessenhofen

Johannes 1406 II 5

Dino

Philippus de Smolna 1412 I 2, 1417 II 5

Dobberkow

Ghiselerus 1401 II, 1403 I

Dobritza (ungar. Kevedobra, ehem. Kom.

Torontál, serb. Dobrica, Serbien) Do-

brica

Clemens 1431 II 13

Do̊rndel

Thomas de Inferiori Altach 1413 II 17

Doleatoris

Johannes 1423 I 22

Dorfen (wahrscheinl. Kr. Erding, RB Oberbayern, Bayern, Dtld.) Dorffen Hilprandus Oder 1429 II 10

Dorpat (russ. Tapty, estn. Tartu, Hauptstadt des Kr. Tartumaa, Estland) Tarbatensis

Bartholomeus Sauigerue 1423 I 23

Drüsselchind

Erasmus 1415 I 10

Drzewiczky

Clemens 1418 I 21

Dvorcze (wohl ungar. Szepesudvard, ehem. Kom. Szepes, slowak. Dvorce, Slowa-

kei) Dworcz

Blasius 1437 I 9
Ebelsbach (wahrscheinl., Kr. Haßberge, RB Unterfranken, Bayern, Dtld.) Ebelspach

Vlricus Lager de Menning 1421 II 6
Ebenhawser

Caspar 1428 II 10. 1431 II 23

Eberhardi

Conradus de Hallis 1410 I 10 
Ebersdorf (nö. Herrengeschlecht) Ebersdorff Johannes 1426 II 16

Eberspoint (Markt Velden, Kr. Landshut (Land), RB Niederbayern, Bayern, Dtld.) Eberspewnt

Heinricus Hůlger 1431 II 16; Petrus Pachmulner 1419 I 21, 1422 I 3, 1424 I 22, 1424 II, 1430 I 25, 1431 I, 1434 II, 1438 I, 1441 II

Ebner

Johannes de Chremsa 1420 I 8

Ebser

Jacobus 1439 I 11, 1439 I 13

Ecker

Nicolaus 1416 II 7

Ëkcherl

Georius 1421 I 3

Egerszeg (entweder Zalaegerszeg, Verwaltungssitz des Kom. Zala, Ungarn, oder Ungarn mehrfach oder Nyitraegerszeg, ehem. Kom. Nyitra, Ungarn, slowak. Jelsovce, Slowakei oder ehem. Kom. Maros-Torda,Ungarn, rumän. Cornățel, Rumänien) Egersek, Egerzeg Georius (Vngarus) 1424 I 10, 1427 II 14, 1430 I 31?, 1430 II 36

Eglinger

Heinricus de Waibling 1417 I 14

Ehingen (Bayern und Baden-Württemberg, Dtld. mehrfach)

Stephanus 1422 II 12

Ehinger

Johannes de Landshůta 1441 II 4

Eyb

Johannes 1423 I 1

Eych s. Aich

Eichstätt (Kr., RB Oberbayern, Bayern,

Dtld.) Astauia, Eystauia, Eystetensis

Conradus Pómer 1437 I 16; Cyriacus Knot 1423 I 18; Conradus Reykershouer (Reykershofer, Reikershofer, Reickershofer) 1430 II 22, 1434 I 19, 1435 I 11, 1435 II 11; Fridericus Warnhofer 1413 I 8; Jodocus Gesler 1421 II 5; Jo- hannes de Eyb 1423 I 1; Johannes de Leonrod 1423 I 17

Eisenach Kr. Eisenach (kreisfr. Stadt, Thüringen, Dtld.) Ysenach, Ysennach

Johannes Hess 1412 I 23, 1423 I 26, 1423 II

Eysenhusen, Ysenhusen (unbestimmt, R)

Johannes Gerhardi 1415 I 9, 1424 II 15

Eysenman

Vlricus de O̊ttinga 1424 I 8

Eystauia, Eistetensis s. Eichstätt

Ekhart

Michael de Ardisch de Septemcastris 1434 I 10

Ekkinger

Bertoldus de Walczhuett 1438 II 6 Elhart

Arbogastus 1433 II 3, 1434 I 16

Elrichshusen

Johannes 1423 II 3

Ellwangen (Jagst, Kr. Ostalbkreis, RB

Stuttgart, Baden-Württemberg, Dtld.)

Elbangen, Elbang, Elwangen

Conradus 1423 I 2, 1426 II 20;

Johannes Hertfelt 1437 II 1 ,

Wernherus (Berngerus) 1430 I 8 , 1433 II 9

Emmen

Conradus de Hildeshaym 1430 II 21

Emmersdorf (GB Spitz, NÖ) Emerstorf

Vrbanus 1416 II 24

Enczestorf Maior s. Großenzersdorf

Enenkel

Marcus de Albrechtzperg 1416 I 17

Engelfrid

Heinricus de Spira 1422 I 12

Enns (GB, OÖ) Anasum

Petrus Chramer 1431 I 2

Eppenstein (Familienname) Eppenstain

Adolffus 1424 I 19

Erbeiter

Johannes 1416 II 31

Erdburg (GB Poysdorf, NÖ) Erdpurg

Bartholomeus Kren 1410 I 5

Erfurt (kreisfr. Stadt, Landeshauptstadt,

Thüringen, Dtld.) Erfordia, Erdfordia 
Conradus Seydenliep 1430 I 2; Theodricus Lobing 1410 I 8

Erhardi (Gerardi)

Wilhelmus de Leydis 1418 II 25, 1420 I 20, 1421 I 7, 1421 I 26

Erlacher

Nicolaus de Purkhawsen 1431 II 5, 1435 I 8

Erlau (ungar. Eger, Verw.-Sitz des Kom.

Heves, Ungarn) Agria, Agriensis

Andreas 1419 II 6; 1423 I 29;

1427 II 11; Clemens 1411 II 15;

Johannes Zimischem 1413 I 25;

Nicolaus Stubner 1430 I 11; bacc. in decr. 1433 I 12; 1435 I 13;

1435 II 13; Oswaldus de Rozgon

1437 I 3; Simon de Bacia 1430 II 6; Stephanus de Abara 1428 II 4; Zegotha archidiaconus de Borswa 1412 II 21
Ermland (Ostpreußen) Warmiensis

Johannes de Hermansdorf 1419 II 11

Ersperg s. Hirschberg

Eschenbach (Bayern mehrfach oder Kr.

Göppingen, RB Stuttgart, Baden-Württemberg, Dtld.)

Hainricus Plesner 1430 I 14

Esslingen am Neckar (Kr., RB Stuttgart,

Baden-Württemberg, Dtld.) Esslinga

Jodocus 1437 II 13, 1440 II 17

Ettenhayn

Petrus de Argentina 1424 II 4

Etter

Petrus 1413 I 14

Etterli

Eglolfus de Bringk in Ergaw 1412 II 10

Ewart

Cristannus de Stolczemberg 1434 I 5

\section{$-\mathrm{F}, \mathrm{V}-$}

Fabri

Erhardus 1430 II 23; Johannes Nicolai de Olsna 1429 II 19; Petrus de Ostrauia 1428 I 2

Vahendorf (unbestimmt)

Fredericus Reschel 1423 I 32

Falkenberg (Burg, Gem. Obervellach, GB Spittal an der Drau, Ktn.)

Andreas 1413 I 5

Falkenstein (GB Langenlois, NÖ) Falkenstain, Falkenstayn

Fridricus Kraft (Krafft) 1406 II 15, 1417 II 6, 1417 II 7, 1418 I 3, 1418 I; Stephanus 1413 I 11

Falkonis

Michael 1417 II 13

Vallis Eni s. Inntal

Vallis Sancte Marie s. Marienthal

Vallis Sancti Georgii s. Gergesthal

Varaw s. Vorau

Varembach s. Formbach

Varichdarff s. Vorchdorf
Varsány (Kom. Nógrád, Ungarn, oder Kisvarsány, Kom. Szabolcs, Ungarn, oder Nagyvarsány, Kom. Szabolcs, Ungarn) Tyssawassan, Tysswassan, Tyzzawazzan

Barnabas 1418 I 9, 1421 I 21, 1422 I 28

Vedröd (ehem. Kom. Pozsony, Ungarn, slowak. Voderady, Slowakei) Vodwat Manussius 1423 I 13

Vey

Johannes de Wratislauia 1421 I 24

Feynockh

Andreas de Berchtoltstorf 1432 I 8

Feyr

Stephanus de Clawsemburg 1431 II 4

Veklaprugk s. Vöcklabruck

Velbêr

Johannes 1410 I 4

Felber

Johannes de Hallis 1413 I 9 
Velberger

Conradus de Hallis 1436 I 2

Feldheim (wohl Kr. Pottsdam-Mittelmark, Brandenburg, Dtld. oder Veltheim Kr.

Halberstadt, Sachsen-Anhalt, Dtld.)

Veltheim

Perchtramus 1416 I 27

Feldkirch (GB, Vorarlberg) Veltkilch

Hermannus Bürser 1409 I 3

Venedig (italien. Venezia, Italien) Veneciae Johannes 1402 I

Veszprim (ung. Veszprém, Ungarn) Vespermiensis, Vesprimiensis, Wesprimiensis

Ladislaus de Hederfar 1434 II 1; Paulus 1415 I 1; Thomas Hym de Brente 1430 I 13

Vetter

Johannes de Memmingen 1414 I 12

Feuchter, Fewchter

Heinricus 1419 II 3, 1430 II 27

Feuchtwangen (wahrscheinl. Kr. Ansbach (Land), RB Mittelfranken, Bayern,

Dtld.) Fewchtwang

Georius Stêninger 1431 II 8

Fëlstein

Johannes de Bladen 1436 II 1, 1441 II 6

Finsterwald(er)

Johannes de Sitauia (Zitauia) 1416 II 6, 1420 II 25, 1422 I 27

Villa Hainperti s. Hammersdorf

Villa Jacobi s. Jakobsdorf

Michael 1437 II 6

Villa Las s. Csütörtökhely oder Szent Laszló

Villa Thobie (unbestimmt) Michael 1437 II 19

Villach (GB, Ktn.) Villacum

Leonardus Rastaker 1421 I 15; Vlricus Reysperger 1419 I 13

Vilseck (Kr. Amberg-Sulzbach, RB Oberpfalz, Bayern, Dtld.) Villseck, Villsegk Andreas Kürsneàr 1439 I 8; 1441 II 8 Fyol

Johannes de Toran 1418 I 15
Virgen (GB Matrei in Osttirol, T)

Erasmus Teisser 1436 II 6

Vischel, Vischlein

Georius 1416 I 8, 1419 I 2

Vischpekch Vischpekh

Gerhardus Vischpekch 1405 Dezember 17, 1405 II 3, 1411 I, 1415 I, 1417 II

Fleckel

Johannes 1438 II 15

Fleckl

Heinricus 1438 II 14

Flemming

Sigismundus de Lantzhuta 1414 I 1

Flieger

Siluester de Augusta 1411 II 12, 1415 II 12 ?

Flokch

Fridericus de Kornburg 1440 II 7

Vodwat s. Vedröd

Vöcklabruck (GB, OÖ) Veklaprugk

Caspar 1434 I 11

Völkermarkt (GB, Ktn.) Volkenmarkt,

Volkenmarckt

Conradus de Chreig 1432 I 1, 1431 II 15

Vohburg a. d. Donau (Kr. Pfaffenhofen a.

d. Ilm, RB Oberbayern, Bayern, Dtld.)

Voburg

Johannes 1410 I 15

Vogel

Nicolaus de Wratislauia 1412 I 3

Vogelsang, Vogelsangk

Jeronimus 1437 I 19, 1439 II 3

Voitsberg (GB, Stmk.) Voytzperg

Leonhardus 1414 I 18

Volkenmarkt, Volkenmarckt s. Völkermarkt

Folczian

Johannes de Wienna 1414 II 1

Vorau (GB Hartberg, Stmk.) Varaw

Nicolaus Czink1433 I 1

Vorchdorf (wohl GB Gmunden, OÖ) Varichdarff

Georius Jågenrểwtter 1428 II 7, 1431 I 12; 1433 II 15

Forchheim entweder Forchheim (Oberfr) 
(Kr., RB Oberfranken, Bayern, Dtld. oder Forchbeim Kr. Emmendingen, Kr. Emmendingen, RB Freiburg i. Br., Baden-Württemberg, Dtld. oder Stadt Freystadt, Kr. Neumarkt i. d. Opf., RB Oberpfalz, Bayern, Dtld.) Folxhaim Johannes de Lapide 1415 I 16 Formbach (Kr. Passau (Land), RB Niederbayern, Bayern, Dtld.) Varembach Conradus 1413 I 22; Georius 1413 I 21

Forum Ruthenorum (unbestimmt, H) Johannes de Wincz 1431 II 12

Frềtinga (unbestimmt, $R$ )

Liebhardus de Ratispona 1416 II 32

Frankfurt am Main (kreisfr. Stadt, RB Darmstadt, Hessen, Dtld.) Francfordia, Franckfordia, Frankfordia, Frankenfordia

Heinricus Scriptoris 1418 II 8; Hermannus Wulco 1417 II 9; Johannes Prunger 1417 II 8, 1418 I 2, 1419 II 16, 1419 II 17; Johannes Rúel 1435 I 3, 1438 I 13, 1441 II 5; Johannes Scriptoris 1413 II 1; Vdalricus 1424 I 20

Frankfurt (Oder) (kreisfr. Stadt, Brandenburg, Dtld.) Frankfordia circa Oderam

Nicolaus Salczmesser 1413 II 18

Vratislauiensis s. Breslau

Fraustadt (poln. Wschowa, Niederschlesien, Polen) Frawnstat

Nicolaus Heroldi 1419 II 1

Fredeberg s. Friedberg

Freiberg (Markt Ebensfeld, RB Oberfranken, Bayern, Dtld.) oder Freiberg am Neckar, Kr. Ludwigsburg, RB Stuttgart, Baden-Württemberg, Dtld.) Friberg Cristannus 1440 II 4

Freyberger Erasmus 1422 II 6

Freiburg im Breisgau (kreisfr. Stadt, RB, Baden-Württemberg, Dtld.) Freyburg Conradus Ruyhing 1411 II 20, 1416 I 30, 1416 II 35, 1417 I
Freyleich

Michael 1438 I 1

Freinstat, Freynstat s. Freystadt

Freising (Kr., RB Oberbayern, Bayern,

$D t l d$.) Frisinga, Frisingensis, Frizyngensis

(Fridericus) de Waldegk 1416 I 2; Hermannus 1416 I 1; Johannes Grünbald 1411 II 13; Johannes Pencznalber 1438 II 8; Deobaldus 1429 II 9, Tieboldus Aichperger 1429 II 14; Wilhelmus Preyzyger 1402 I 1

Freistadt (GB, OÖ) Freynstat

Wolfgangus Wurm 1414 I 6, 1437 II 15

Freystadt i. Niederschles. (poln. Kożuchów, Niederschlesien, Polen, oder Freistadt tschech. Fryštát zu Karviná, GB, Schlesien, Tschechien, oder Freistadl ungar. Galgóc, ehem. Kom. Nyitra, slowak. Hlohovec, Slowakei) Freinstat, Freynstat

Johannes Sutoris 1437 II 2, Stephanus Neumann 1430 II 9

Freythơfl

Wolfgangus de Gmunden 1438 I 10

Friberg s. Freiberg

Fridwalt

Caspar de Nissa 1416 I 23

Friedberg (Hessen) (Kr. Wetteraukreis, RB Darmstadt, Hessen, Dtld. oder Friedberg Kr. Aichach-Friedberg, Kr. Aichach-Friedberg, RB Schwaben, Bayern, Dtld. oder Friedberg Stadtteil von Bad Saulgau, Kr. Sigmaringen, RB Tübingen, Baden-Württemberg, Dtld.) Fridwerkch, Fredeberg Conradus Reyndel 1422 II 9; Johannes Poter Sartoris 1429 I 15

Friedland (Brandenburg, Mecklenburg, Ostpreußen, Tschechien mehrfach)

Fridland, Fridlang

Nicolaus Hermanni 1424 I 16, Theodricus 1429 II 17

Frieshaymer

Jacobus de Schirling 1422 II 13 
Friesoythe (Kr. Cloppenburg, RB WeserEms, Niedersachsen, Dtld.) Oyta Gotfridus (Fryling) 1412 II 1, 1415 I 13, Heinricus 1414 I 5; Martinus 1412 II 2

Frigidus Fons s. Kaltenbrunn

Fryling

Gotfridus de Oyta 1412 II 1, 1415 I 13

Frisinga, Frisingensis, Frizyngensis $s$.

Fuchs Freising

Johannes de Danczk 1425 I 4; Petrus 1431 II 10,1433 II 11
Füchtensteiner

Antonius 1413 II 5

Fünfkirchen (ung. Pécs, Stadt im Komitatsrang und Verw.-Sitz des Komitats Baranya, Ungarn) Quinque Ecclesiae, Quinqueeclesiae, Quinqueecclesiensis Ciriacus 1429 I 7; Mathias 1423 I 15; Simon de Bacia 1430 II 6 Fưssel, Fưssil, Fưszel

Johannes de Bratislauia (Wratislauia) 1411 II 9, 1416 I 28, 1420 I 16, 1420 II 24

Furenrider

Thomas de Amberga 1418 II 21
Gamezii (Gomezii)

Alfonsus 1424 II 5, 1428 II 14, 1430 I 23, 1430 I 24

Gamundia s. Gmünd

Garsten (GB Steyr, OÖ) Gersten Sigismundus de Styra 1425 II 1

Gartner Johannes de Perching 1419 I 14

Gaspoltshofen (GB Haag am Hausruck, $O O ̈)$ Gastpoltshouen Johannes Schiltdorffer 1430 I 4

Gastmeister, Gastmeyster Heinricus de Gobin 1415 I 2, 1417 I 8

Gawen

Johannes de Lenicz 1412 I 16

Gebhardi Johannes 1415 II 2

Geysenuelder Leo de Ratispona 1420 II 19

Gelesdorff, Gelestorf, Gelestorff s. Göllersdorf

Gelprecht, Geltprecht Johannes de Lintz 1438 II 1, 1440 II 16

Gemundia s. Gmünd

Gerardi s. Erhardi

Gergesthal (ungar. Szent Györgyválya, ehem. Kom. Hunyad, rumän. Valea
Sângeorgiului, Rumänien) Vallis Sancti Georgii

Gregorius 1435 I 2, 1437 II 16, 1439 II 7,1439 II 9

Gerhardi

Johannes de Eysenhusen (Ysenhusen) 1415 I 9, 1424 II 15

Gern (Bayern, Dtld. mehrfach) Geren

Andreas Chlosner 1415 II 1

Gersten s. Garsten

Gesler

Jodocus 1421 II 5

Gewalt

Vlricus de Newmburga 1421 II 12

Gewitsch (tschech. Jevičko, Mähren, Tschechien) Gewicz

Johannes Ruschlini alias Judicis 1416 I 16

Giech (Stadt Scheßlitz, Kr. Bamberg (Land), RB Oberfranken, Bayern, Dtld.) Gich, Gich

Demetrius 1414 II 2; Theodorus 1415 I 5

Giesser

Johannes de Monaco 1440 II 6 Gilgenburg (poln. Dąbrówno, einst Kr. Osterode, Ostpreußen, Polen, viell. Gilgenberg Gem. Waldkirchen an der Thaya, GB Laa an der Thaya., NÖ 
oder Gilgenberg am Weilhart, GB

Braunau am Inn, OÖ)

Jacobus 1412 I 9

Girolt (ehem. Kom. Szolnok-Doboca,

Ungarn, rumän. Ghirolt, Rumänien)

Gyrold

David 1428 II 9

Gladiatoris

Philippus 1433 II 2

Glatz (poln. Ktodzko, Niederschlesien, Po-

len) Glocz

Nicolaus (Stůrpecher) 1434 I 7, 1440 I 5, 1440 II 12

Glawbis

Bartholomeus 1416 II 16

Glen

Paulus de Noua Ciuitate 1431 I 8

Gleiwitz (poln. Gliwice, Oberschlesien,

Polen) Gleybicz, Gleybitz

Paulus Cracouiensis 1436 I 1, 1438 II 20, 1442 I 8

Glocz s. Glatz

Glőkelherr

Johannes 1408 I 2

Glogau (Groß-) (poln. Gtgów, Niederschlesien, Polen) Glogouia, Glogouia Maior, Glogouiensis Maior

Georius Liechtenberg 1412 I 24; Marcus Lessna 1434 I 8; Nicolaus Heroldi de Frawnstat 1419 II 1; Nicolaus Stok (Stokch, Stock)

1413 I 17, 1421 I 20, 1422 I 25; 1422 II

Gloting

Nicolaus 1419 I 22

Gmünd (entweder Gmünd, mehrfach in Österreich od. Gmünd, mehrfach in Süddeutschland od. Schwäbisch Gmünd, Kr. Ostalbkreis, BadenWürttemberg, Dtld.) Gamundia, Gemundia

Johannes 1421 II 13; Martinus Back 1410 II 4

Gmunden (GB, OÖ) Gmvnden Johannes 1427 II 1; Wolfgangus Freytho̊fl 1438 I 10
Gnès

Jacobus de Sancto Vito 1414 I 23

Gnechowicz Johannes de Wratislauia 1411 I 2

Gnendorffer Johannes Gnendorffer 1422 I 13

Gnesen (poln. Gniezno, Bez. Posen, Polen)

Gnyznå, Gnesensis

Bernhardus Lang 1419 I 11; Mathias 1418 I 22

Gobin, Gobyn s. Guben

Goch (Kr. Kleve, RB Düsseldorf, Nordrhein-Westfalen, Dtld.) Goth

Erasmus Kirchhof 1442 I 5

Godke

Johannes de Wratislauia 1418 I 14

Gödke

Johannes de Danczk 1412 I 4

Göllersdorf (GB Hollabrunn, NÖ) Gelesdorff, Gelestorf, Gelestorff

Thomas 1423 I 5, 1424 II 12, 1437 II 22

Göppingen (Kr., RB Stuttgart, Baden-

Württemberg, Dtld.) Göpping

Michel 1424 II 1

Görlitz (kreisfr. Stadt, RB Dresden, Sachsen, Dtld.; östl. Stadtteile unter dem

Namen Zgorzelec, Polen) Gorlicz,

Gơrlit

Johannes Stange 1432 II 7; Petrus

Bartholomei 1437 I 10

Göß (Gem. Leoben, Stmk.) Goss

Petrus Hebringer 1430 I 10

Göttweig (GB Krems an der Donau, NÖ)

Gotwicum

Johannes 1442 I 2

Goldberg (poln. Ztotoryja, Niederschlesien, Polen) Goltperg

Johannes (Streit) 1435 II 9, 1437 II 20

Gollub (Stadt nordöstl. von Thorn, poln.

Golub-Dobrzyń, Polen) Golaw

Ambrosius Neczowencz 1417 I 16

Goltperg

Nicolaus (Mathie) de Wratislauia 1410 I 14, 1416 II 38

Gomecii s. Gamezii 
Gora (viell. ehem. Kom. Zágráb, Ungarn, heute Kroatien)

Mathias Cordobok 1411 I 4; Nicolaus Sartoris 1411 I 7

Gorlicz, Goorlit s. Görlitz

Goss s. Göß

Goth s. Goch

Gotwicum s. Göttweig

Gotz

Johannes de Studkardia 1429 II 24

Grafendorf (mehrfach in Ö) Greàendorff Stephanus Johannis 1422 I 7

Grafenwörth (GB Tulln, NÖ) Grauenwerd

Johannes de Rosenaw 1421 I 11

Grayspach

Wilhelmus de Monaco 1428 II 6

Gramus

Nicolaus 1422 II 1

Gran (ungar. Esztergom, Kom. Komorn,

Ungarn) Strigonia, Strigonium, Strigoniensis

Albertus de Waradino (de Baradino) 1428 I 3, 1430 II 30, 1432 II 11, 1434 II 16; Andreas de Labatlan (Labathlan) 1435 II 10, 1439 II 2, 1440 II 11; Antonius de Busa 1431 II 3; Caspar 1430 II 25; Gregorius de Valle Sancti Georgii 1435 I 2, 1437 II 16, 1439 II 7, 1439 II 9; Gregorius Grunt 1416 I 22; Jacobus 1432 II 10, 1434 II 8, 1434 II 12; Johannes 1427 II 2

Granatoris

Franciscus de O̊ttingen 1410 II 1

Grassau (Kr. Traunstein, RB Oberbayern, Bayern, Dtld.) Grassaw

Nicolaus Hêppel 1420 II 7

Grassertal s. Grossturwall

Graudencz

Nycolaus 1411 I 14

Grauenwerd s. Grafenwörth

Grauscharn (heute Pürgg-Trautenfels, GB Irdning, Stmk.) Grawscharn

Ernestus Mèssemperger 1421 II 11
Graw

Johannes 1432 II 2

Grawscharn s. Gratschach

Graz (Landeshauptstadt, Stmk.) Grẻcz, Grètz

Andreas 1438 I 9, Wolfgangus 1438 I 4

Grềtzer

Stephanus 1410 I 19

Grewätzensteten s. Kreuzstetten

Greifswald (kreisfr. Stadt, MecklenburgVorpommern, Dtld.) Gribiswalda,

Gribswalda

Andreas Burmeyster 1416 I 13; Erasmus Rubennaw 1418 II 20

Grenn

Fridericus de Rotemberga 1416 II 9

Grecz Regine s. Königgrätz

Gribiswalda, Gribswalda s. Greifswald

Griffen (GB Völkermarkt, Ktn.) Grifen,

Griuen

Georius de Zůmelsperg 1414 I 9; Hertwicus de Zumelsperg 1421 I 2

Großau (ungar.Keresténysziget, ehem. Kom. Szeben, rumän. Cristian, Siebenbürgen, Rumänien) Insula Christiana Michael (de Septemcastris) 1418 II 26, 1438 II 18

Großenzersdorf (GB, NÖ) Enczestorf Maior Johannes Gebhardi 1415 II 2

Großschenk (ungar. Nagysink, rumän. Cincu, Siebenbürgen, Rumänien) Schenck Maior Johannes 1421II 4

Groß-Schweinbart (GB Gänserndorf, NÖ) Sweinbart

Pangracius Conradi 1430 I 6 Grossturwall (viell. ungar. Törökbálint, Kom. Pest-Pillis-Solt-Kiskun, Ungarn) Grassertal Cristannus Murher 1412 I 5

Großwardein (ungar. Nagyvárad, rumän. Oradea, Rumänien) Waradinum, Waradiensis, Baradinum, Baradiensis Albertus 1428 I 3, 1430 II 30, 
1432 II 11, 1434 II 16; Benedictus 1422 II 11; Johannes de Tapolcza 1434 I 1, 1436 I 5 , 1438 I 12, 1438 I 17; Ladislaus de Schlak 1427 II 5; Nicolaus (de Ungaria) 1414 I 7, 1417 II 2, 1419 II 23, 1421 I 19

Gruber

Leonardus 1420 I 4

Grůen

Johannes de Hallis 1441 I 9

Grünbald

Johannes 1411 II 13

Grunt

Gregorius de Strigonia 1416 I 22

Guben (Kr. Spree-Neiße, Brandenburg,

Dtld.) Gobin, Gobyn

Heinricus Gastmeister (Gastmeyster) 1415 I 2, 1417 I 8

Günzburg (Kr., RB Schwaben, Bayern,

Dtld.) Gvnczpurg

Johannes Ambrosii 1423 I 19, 1426 I

11
Gưrtesch s. Kürtös

Gugk

Bertoldus 1438 II 21, 1442 I 12

Guldein

Cristoferus (de Rain) 1423 I 24, 1426 II 19

Gůt

Johannes de Corona 1437 II 8, 1440 I 8

Gurkfeld (ehem. Land Krain, Bez. Gurk-

feld, slowen. Krško, Slowenien) Gurkchueld

Georius Ëkcherl 1421 I 3

Gurnitz (GB Klagenfurt, Ktn.) Gurnocensis

Johannes Årkenstainer 1424 I 5

Gwerleich, Gwårleich

Johannes 1406 II 17, 1419 II 21, 1421 I 5, 1421 I,1426 I, 1430 I, 1432 I, 1435 II
Habelschwerdt (poln. Bystrzyca Ktodzka, Niederschlesien, Polen) Habelswerd Martinus 1420 II 13

Hadersdorf (mehrfach in Ö) Hederstorff,

Headersdorff

Wolfgangus Payer (Payr) 1421 I 9, 1430 I 26

Hågg

Johannes de Kirchain 1424 I 3

Håntinger

Vlricus plebanus in Bischofst(orf) 1440 I 4

Håppel

Cristannus 1420 I 1; Heinricus 1426 I 3, Nicolaus de Grassaw 1420 II 7

Johannes 1419 II 13

Haez s. Hers

Haidelshaim s. Heidelsheim

Haydendarff s. Heidendorf
Haydenhaym s. Heidenheim

Haydreichstain s. Heidenreichstein

Halberstadt (Kr, RB Magdeburg, SachsenAnhalt, Dtld.) Halberstat

Henningus 1414 I 22

Halla s. Schwäbisch Hall

Hallein (GB, Sbg.) Salina

Cristannus Wurm 1412 I 20; Oswaldus 1418 I 19

Hallstatt (GB Bad Ischl, OÖ) Halstat

Conradus 1414 II 10, 1419 I 3, 1422 I 23, 1423 I, 1427 II, 1429 II, 1434 I, 1436 II, 1440 II; Johannes 1433 I 6

Hamelburg s. Hammelburg

Hammersdorf (ungar. Szenterzsébet, ehem.

Kom. Szeben, rumän. Gussterita, Sie-

benbürgen, Rumänien) Villa Hainperti

Cristannus 1430 II 15, 1433 II 8, 1436 I 7, 1436 II 13 
Hamlesch (ungar. Omlás, ehem. Kom. Szeben, Ungarn, rumän. Ámláse, Siebenbürgen, Rumänien) Hamlach, Omlaschz

Antonius, Anthonius (de Septemcastris) 1430 II 16, 1432 II 9, 1436 II 9, 1436 II 12

Hammelburg (Kr. Bad Kissingen, RB Unterfranken, Bayern, Dtld.) Hamelburg

Heinricus Stoll 1414 I 15; Petrus 1426 II 15

Hannenbach (ungar. Glimboka, ehem. Kom. Szeben, Ungarn, rumän. Glâmboaca, Siebenbürgen, Rumänien oder ungar. Kakasfalva, ehem. Kom. Szeben, Ungarn, rumän. Hamba, Siebenbürgen, Rumänien) Hanebach Symon 1423 II 5

Harscher Gotfridus 1411 II 19

Harster Johannes de Lantzhuta 1416 I 18

Hartberg (GB, Stmk.) Hartperg Wolfgangus 1416 II 2

Has $s$. Hers

Haßfurt (Kr. Haßberge, RB Unterfranken, Bayern, Dtld.) Hasfordia Chonradus Schober 1416 II 34

Hau (viell. Nordrhein-Westfalen, mehrfach oder Gem. Landscheid, Kr. Bernkastel-Wittlich, RB Trier, Rheinland-Pfalz, Dtld. oder Stadt Calw, Kr., RB Karlsruhe, BadenWürttemberg, Dtld.) Hew Heinricus 1421 II 9

Haug (Stift in Würzburg, kreisfr. Stadt, RB Unterfranken, Bayern, Dtld.) Hawg, Håwg Bertoldus Tebershaymer 1425 I 5; Johannes Erbeiter 1416 II 31

Hausdorf (Österreich mehrfach) Jeronimus Posser (de Salczburga) 1436 II 3, 1439 II 4

Hawnsperger

Herdwicus 1410 I 16

Hawsecker Vlricus 1409 I 5
Hawsner

Jodocus (de Nouoforo) 1437 I 14, 1440 I 10

Vlricus 1402 I 6

Hềler

Gebso de Constancia 1426 I 2

Hewndel

Hebringer

Petrus 1430 I 10

Hecht

Petrus de Künigsperg 1413 I 1; 1416 I 29

Hèdersdorff, Hedersdorff s. Hadersdorf

Hedervár (Kom. Györ, Ungarn) Hederfar

Ladislaus 1434 II 1

Heidelsheim (Stadtteil von Bruchsal, Kr.

Karlsruhe (Land), RB Karlsruhe, Ba-

den-Württemberg, Dtld.) Haidelshaim

Hermannus 1413 I 16

Heidendorf (viell. ungar. Besenyö, ehem.

Kom. Beszterce-Naszód, rumän.

Viişoara, Rumänien oder ungar.

Székásbesenyö, ehem. Kom. Alsó-Feher, rumän. Secăşel, Rumänien) Hayden-

darff

Vicencius 1430 II 10

Heidenheim an der Brenz (Kr., RB Stuttgart, Baden-Württemberg, Dtld.) Haydenhaym

Vlricus Nernshaymer 1415 II 6

Heidenreichstein (GB Litschau, NÖ) Haydreichstain

Vdalricus 1431 II 1

Heylsmar

Michel de Stolczemburg 1418 II 16, 1422 I 29, 1435 I 12, 1435 II 16

Heimbach (Süddtld. mehrfach) Heympach Petrus 1422 II 4

Heinrici

Nicolaus de Praga 1424 II 8

Hekch

Petrus de Reyspach 1423 I 16

Hellenbach (wahrscheinl. ungar. Helemba, ehem. Kom. Hont, slowak. Chlaba, Slowakei) Helpach

Fridericus Purger 1419 I 20

Heller

Johannes de Monaco 1437 I 15 
Helman

Martinus 1416 II 29

Helmstadt Kr. Würzburg (Kr. Würzburg

(Land), RB Unterfranken, Bayern,

Dtld. oder Helmstadt-Bargen, Kr.

Rhein-Neckar-Kreis, RB Karlsruhe,

Baden-Württemberg, Dtld.) Helmstat

Chunradus 1409 I 1; Heinricus

1413 I 15; Reynhardus 1412 I 7

Helpach s. Hellenbach

Heltau (ungar. Nagydisznód, ehem. Kom.

Szeben, rumän. Cisnădie, Siebenbür-

gen, Rumänien) Helta, Heltaw

Bertoldus 1415 II 11; Nicolaus

1416 II 30; Petrus 1415 I 7

Heltzdorf (ungar. Höltövény, ehem. Kom.

Brassó, rumän. Hălchiu, Burzenland,

Rumänien) Hiltensdorff

Cristannus de Wurcia 1429 II 20

Helwich

Tylmannus 1421 II 14

Hemau (Kr. Regensburg (Land) RB Oberpfalz, Bayern, Dtld.) Ornbawr

Paulus Meck 1439 I 7

Henckel

Petrus 1416 II 15

Henrici

Conradus de Hildesheim (Hildensem) 1407 II 2, 1414 I 31

Herbelstat s. Herbstadt

Herbeyn

Conradus de Tywing 1426 I 5

Herbipolis, Herbipolensis s. Würzburg

Herbstadt (viell. Kr. Rhön-Grabfeld, RB

Unterfranken, Bayern, Dtld.)

Michel 1412 I 17

Hercz

Narciscus 1410 I 7

Herdlof (unbestimmt, S)

Petrus Absolonis 1419 II 2

Heresinger

Johannes de Strawbinga 1422 II 8

Heridensis s. Herrieden

Hermanni

Dominicus de Slesia 1437 II 4; Nicolaus de Fridlang 1424 I 16

Hermannstadt (ungar. Nagyszeben, rumän. Sibiu, Bez. Kronstadt, Sie-

benbürgen, Rumänien) Cibinium,

Cibiniensis

Johannes 1406 II 12, Symon de Rozgon 1413 I 4

Hermersdorf (viell. Ortsteil von Mün-

cheberg, Kr. Märkisch-Oderland,

Brandenburg, Dtld.) Hermansdorf,

Hermansdorff

Johannes 1419 II 11; Mathias

$$
1420 \text { II } 5
$$

Heroldi

Nicolaus de Frawnstat 1419 II 1

Herr

Kaspar de Vlma 1411 II 11

Herrant, Herrannt

Erhardus (de Newmburga Claustrali) 1422 I 20, 1425 II 3, 1430 I 30, 1430 II, 1432 I 15, 1433 II

Herrenberg (Kr. Böblingen, RB Stuttgart,

Baden-Württemberg, Dtld.) Herrem-

berg

Johannes 1414 II 6

Herrieden (Kr. Ansbach (Land), RB Mittelfranken, Bayern, Dtld.) Herriden, Heridensis

Erhardus Fabri 1430 II 23; Mathias

Regelshofer (Regelshoffer Regelshouer) 1408 I 3, 1410 I 18, 1419 I

Hers (Hẽz, Has)

Johannes (de Brunna) 1429 I 1, 1432 I 20, 1438 I 14

Hertfelt

Johannes de Elwangen 1437 II 1

Herzogenburg ( $G B, N O ̈)$ Herczogemburg,

Herczogburg, Herczogburga

Georgius 1435 II 1, 1440 II 18, 1435 II 1; Lodowicus 1423 I 8; Petrus 1420 I 14

Hess

Johannes de Ysennach 1412 I 23, 1423 I 26, 1423 I 27, 1423 II

Heympach s. Heimbach

Hieler

Johannes de Ala 1429 II 23

Hiersberg s. Hirschberg 
Hildesheim (Kr., RB Hannover, Niedersachsen, Dtld.) Hildeshaym, Hildensa Conradus Emmen 1430 II 21; Conradus (Henrici) 1407 II 2, 1414 I 31

Hylsmar s. Heylsmar

Hym

Thomas de Brente 1430 I 13

Himberg (wohl Teil von Wien 23) Hymperg

Bartholomeus Menseer 1423 I 7

Hymel

Johannes de Weytz 1411 I 8

Hiltenstorff s. Heltzdorf

Hindernpach

Mag. Johannes Hindernpach 1439 I 10

Hipples (Gem. Großrußbach, GB Korneuburg, NÖ) Hyppleins

Colomannus Knapp1422 I 1, 1425 II 7, 1428 II 12, 1430 II 33

Hirschberg (in Ostdtld. mehrfach; wahrscheinl. Hirschberg im Riesengebirge, poln. Jelenia Góra, Niederschlesien, Polen) Hiersberg, Ersperg

Bartholomeus Czedlicz 1423 II 7; Petrus Stang 1416 I 4

Höflein (NÖ, OÖ und Stmk. mehrfach)

Ho̊flein

Conradus Riedrer 1437 II 3

Hofeman

Jacobus de Onalbach 1405 II 1

Hoffkircher

Johannes de Wienna 1426 I 4

Hoffner

Vlricus 1432 I 4

Hofleich

Johannes (de Vlma) 1427 II 9, 1432 I 19

Hofrichter

Johannes de Hallis 1424 I 12

Hohenberg (Bayern und Baden-Württemberg, Dtld. mehrfach) Hohemberg

Wigandus Menkler 1423 I 3

Hohenloch

Georgius de Bamberga 1435 I 1
Hohenmauth (tschech. Vysoké Mýto, Böhmen, Tschechien) Altamuta

Johannes 1432 I 10

Hollabrunn (GB, NÖ) Holabrunna

Michael 1425 I 6

Hollenburg (wohl Krems an der Donau,

GB, NÖ oder Gem. Köttmannsdorf,

GB Klagenfurt, Ktn.) Holenburg

Vincencius Weinstok 1409 II 8

Holer

Oswaldus Holer de Valle Eni 2 gr. 1421 II 10

Holzhausen (Bayern, Baden-Württemberg,

Dtld. mehrfach) Holczhausen

Vlricus Horlactawer de Monaco 1411 II 1

Holczman

Johannes de Aw 1416 II 23

Horant

Vlricus Horant (de Sweinfordia)

1439 I 6; 1439 I 12

Horlactawer

Vlricus de Monaco 1411 II 1

Hoszil

Petrus 1417 I 2

Hotzenplotz (tschech. Osoblah, Schlesien,

Tschechien) Hoczemplocz

Nicolaus Kalman 1423 I 4

Hublinger

Martinus de Wels 1415 II 14

Hülger

Heinricus de Eberspewnt 1431 II 16 Hümel

Petrus de Petouia 1439 I 2

Hürnheim (Gem. Ederheim, Kr. Donau-

Ries, RB Schwaben, Bayern, Dtld.)

Hurnhain Hürnhayn

Wilhelmus 1413 I 6, 1417 I 18

Hunyad

Martinus 1428 II 8, 1430 I 32

Husel

Johannes 1411 II 4

Huxer

Hermannus de Pruscia 1430 II 1 
Jågenrewảtter, Jågenreiter, Jegenrewtter Georius de Patauia 1428 II 7, 1431 I 12, 1433 II 15

Jakobsdorf (entweder ungar. Gyákos, ehem. Kom. Nagy-Küküllö, rumän. Giacăş, Siebenbürgen, Rumänien oder ungar. Jakabfalva, ehem. Kom. Nagy-Küküllö, rumän. Iacobeni, Siebenbürgen, Rumänien oder ungar. Szászentjakab, ehem. Kom. Szolnok-Doboka, rumän. Sâniacob, Siebenbürgen, Rumänien) Villa Jacobi

Michael 1437 II 6

Jauer (poln. Jawor, Niederschlesien, Polen) Jawr, Jauriensis

Thomas de Norap 1431 II 11; VrbaJax nus (Sculteti) 1438 I 3, 1442 I 14

\section{Dyonisius 1414 I 24}

Idstein (Kr. Rheingau-Taunus-Kreis, RB Darmstadt, Hessen, Dtld.) Ytstein Bruno Trudelonis 1417 I 15 Jechnitz (tschech. Jehnice, GB Brünn-Umgebung, Mähren, Tschechien) Lenicz Johannes Gawen 1412 I 16 Jeger

Johannes 1429 I 16

Jenkebicz

Nicolaus de Woslawicz 1429 I 4 Yencz

Johannes de Wratislauia 1419 I 4

Jerxheim (viell. Kr. Helmstedt, RB Braunschweig, Niedersachsen, Dtld.) Jerucensis Johannes 1417 I 12, 1422 I 18

Jeskonis

Michel de Resinkirche (Rosenkirch) 1411 I 1, 1413 I 19

Jewkewicz

Johannes de Possilwicz 1422 II 5

Im Wald (unbestimmt, $A$ )

Florianus de Sancto Floriano 1435 II 8

Ymmerzel (unbestimmt)

Olyuerus 1438 I 5
Inferior Altach s. Niederaltaich

Inferior Stokchstal s. Unterstockstall

Ingolstadt (Kr., RB Oberbayern, Bayern,

Dtld.) Ingolstat

Michel Phêffel 1418 II 24

Innichen (italien. San Candido, Südtirol, Italien) Inticensis

Leonardus Rastaker de Villaco 1421 I 15

Innsbruck (Landeshauptstadt, T)Ynsprucka Jodocus Mêntelberger 1422 I 15

Inntal (Tirol, Österreich) Vallis Eni

Erasmus 1406 II 9; Heinricus 1406 II 10; Oswaldus Holer 1421 II 10

Institoris

Georius de Lobyn 1419 II 9, 1421 II 17

Insula Christiana s. Großau

Inticensis s. Innichen

Joel

Tilmannus de Lyris 1413 II 8

Johannis

Franciscus de Luko 1425 I 3; Jacobus de Mùsson 1422 I 5; Nicolaus de Dacia 1420 II 10; Nicolaus de Frigido Fonte 1418 I 6; Stephanus de Grẻuendorff 1422 I 7

Jonsdorf

Johannes 1412 I 25

Jordansmühl (poln. Jordanów Ślaski, Niederschlesien, Polen) Jordansmül

Vincencius 1418 I 4, 1420 I 19, 1421 I 6

Ysenach, Ysennach s. Eisenach

Ysenhütel

Nicolaus de Wienna 1413 II 11

Ysenhusen s. Eysenhusen

Ytstein s. Idstein

Judicis

Johannes Ruschlini de Gewicz 1416 I 16

Jukarim (unbestimmt, S.)

Item Conradus Phơrtner de Jukarim dt. 2 gr. 1412 I 6 
Jung

Dauid 1414 I 26

Laa an der Thaya (GB, NÖ) oder Oberbzw. Unterlaa, Wien 10

Petrus (Newsidler) 1426 II 6, 1432 II 12

Labod (Kom. Somogy, Ungarn) Labatlan, Labathlan

Andreas 1435 II 10, 1439 II 2, 1440 II 11

Lachmair

Vlricus 1431 I 3

Lager

Vlricus de Menning 1421 II 6

Laibach (slowen. Ljubljana, Slowenien)

Laybacum

Andreas 1431 I 5; Johannes Czepeck (Zepeck) 1413 I 13, 1431 II 25

Layminger

Georius 1406 II 7; Leonardus 1407 II 6

Lamberg

Johannes de Wratislauia 1420 II 4

Lampoltinger, Lampotinger

Hartwicus (Hertwicus) 1431 I 1, 1435 II 19, 1438 I 16, 1438 I 18

Lanczans s. Landshut in Mähren

Landestrost

Gregorius 1414 II 15

Landfoyd, Lantuoyt

Jeronimus de Olmüncz (Olmuncz) 1428 I 6, 1430 I 33, 1433 II 14

Landshut (Kr., RB Niederbayern, Bayern, $D$ tld.) Lantzhout, Landshůta, Lantshuta, Lantzhuta

Caspar (Westerndorffer) de Piburga 1433 II 16, 1436 I 6; Chunradus Wirsung 1411 I 10; Erasmus 1417 I 4; Johannes Ehinger 1441 II 4; Johannes Harster 1416 I 18; Johannes Vbelman 1416 I 3; Petrus 1426 II 3; Sigismundus Flemming 1414 I 1
Landshut in Mähren (viell. tschech. Lanžhot, GB Břeclav, Mähren, Tschechien) Lanczans Jacobus 1409 I 7

Lang Bernhardus 1419 I 11; Gabriel (de Augusta) 1435 I 6; Vdalricus de Augusta 1429 II 13, 1433 I 5

Langkhaim

Andreas de Prussia 1428 II 2

Langstadt (Stadtteil von Babenhausen [Hess], Kr. Darmstadt-Dieburg, RB Darmstadt, Hessen, Dtld.) Langstat Conradus 1422 II 3, 1424 II 14

Lankow Luwertus de Danczk 1424 I 15

Lantshuta, Lantzhout, Lantzhuta s. Landshut

Lapicide

Johannes de Kunigshofen 1437 II 5

Lapis s. Stein

Laskowa (Galizien, Polen) Lascho Bohoslaus 1421 I 13

Lauben Kr. Oberallgäu (Kr. Oberallgäu, RB Schwaben, Bayern, Dtld. oder Lauben Kr. Unterallgäu, Kr. Unterallgäu, RB Schwaben, Bayern, Dtld.) Lawben Conradus Scharb 1412 II 20

Lauffen (Gem. Bad Ischl, GB, OÖ) Lawffen

Hainricus 1408 II 3

Lautersheim (Kr. Donnersbergkreis, RB Rheinhessen-Pfalz, Rheinland-Pfalz, Dtld.) Lawtershaym Arnoldus 1415 I 15

Lawffen s. Lauffen

Lawtershaym s. Lautersheim Lechling s. Bechlingen

Ledenter Gregorius 1412 II 5 
Lehener

Johannes de Purchhawsen 1423 I 11

Leibitz (ungar. Leibic, slowak. L'ubica, Slowakei)

Martinus 1429 II 3

Leibnitz (GB, Stmk.)

Florianus 1430 I 12

Leiden (Niederlande) (Leyda)

Wilhelmus Erhardi (Gerardi) 1418 II 25, 1420 I 20, 1421 I 7, 1421 I 26

Leinstetten (Stadtteil von Dornhan, Kr. Rottweil, RB Freiburg im Breisgau, Baden-Wörttemberg, Dtld.) Leynsteten Hugo 1420 II 11

Leipa (tschech. Lipa, GB Königgrätz, Böhmen, Tschechien) Lyppa

Matheus Andree 1441 I 7

Lenicz s. Jechnitz

Leoben (GB, Stmk.) Lỏben, Lewben, Lobyn

Georius Institoris 1419 II 9, 1421 II

17; Johannes Wücherl 1418 II 22

Leobschütz (poln. Gtubczyce, Oberschlesien, Polen) Lủbschütz

Egidius Scherffemberg 1442 I 4

Leonrod (Markt Dietenhofen, Kr. Ansbach (Land), RB Mittelfranken, Bayern, Dtld.)

Johannes 1423 I 17

Lepes

Nicolaus 1434 II 2, 1437 II 17, 1439 II 6,1439 II 8

Lessna

Marcus de Maiori Glogouia 1434 I 8

Leupolt

Ludouicus de Monaco 1442 I 15

Lewben s. Leoben

Lewbner

Johannes de Nova Civitate 1420 I 6; Laurencius filius Nicolai de Nissa 1431 II 6

Lewprechtinger

Bernhardus 1437 I 17; 1440 II 14

Libnicensis s. Liegnitz

Lichtenberg (mehrfach in Bayern und Hessen, Dtld.) Liechtenburg
Petrus O̊denfurter 1431 I 4

Liebenstein (Stadt Kötzting, Kr. Cham, RB Oberpfalz, Bayern, Dtld. oder Markt Plößberg, Kr. Tischenreuth, RB Oberpfalz, Bayern, Dtld.) Lybenstain Rabanus 1430 I 16

Liebhardi

Michel 1419 I 23

Liechtenberg

Georius 1412 I 24

Liechtenburg s. Lichtenberg

Liechtenuelder

Thomas 1410 I 1

Liegnitz (poln. Legnica, Niederschlesien, Polen) Legnicz, Legnitz, Libnicensis

Franciscus (Woiczdorff) 1429 I 2, 1430 I 29, 1434 II 14, 1434 II

15; Paulus Mankelwicz 1430 II 2

Limpurg (schwäb. Adelsgeschlecht; Reichserbschenken von Limpurg) Lymburg

Fridericus Schenkch 1411 II 8

Lind (mehrfach in Bayern, Nordrhein-

Westfalen und Rheinland-Pfalz, Dtld.)

Lind

Johannes Wollflein 1410 I 9

Lynss s. Linz a. Rhein

Linz (Landeshauptstadt, OÖ), Lincz,

Lintz, Lynntz

Johannes Geltprecht (Gelprecht) 1438 II 1, 1440 II 16; Petrus Mürbacher 1426 II 2

Linz a. Rhein (Kr. Neuwied, RB Koblenz, Rheinland-Pfalz, Dtld.) Lynss, Lyris

Petrus de Caldario 1416 I 11; Tilmannus Joel 1413 II 8

Lipan (Böhmen, Tschechien mehrfach oder Lippa ungar. Lippa, ehem. Kom. Temes, Ungarn, rumän. Lipova, Rumänien) Lyppa s. auch Leipa

Matheus Andree 1441 I 7

Lyppa s. Leipa

Lyris (wohl Verschreibung aus Linz; s. Linz a. Rhein)

Lobing

Theodricus de Erdfordia 1410 I 8

Lobyn s. Leoben 
Loman

Johannes de Riga 1418 I 13

Ludberti

Johannes 1415 II 8

Lủbersdorp

Henningus 1414 I 13

Lủbschủtz s. Leobschütz

Lücko s. Eukow

Luf

Jacobus de Weyssemburga 1422 II 7

Łukow (Bez. Lublin, Polen oder Eukowa
Bez. Lublin, Polen) Luko, Lutko,

Lukau

Franciscus Johannis 1425 I 3; Johannes Rademan 1430 I 5, 1434 I 18; Johannes Tornaw 1411 II 10, 1420 II 26

Lurczer

Thomas 1413 II 2

Lusning

Cristoferus 1422 I 11

Lutko s. Eukow
Maastricht (Hauptstadt der Prov. Limburg, Niederlande) Traiectum, Traioctensis Nicolaus Cluting 1419 I 22, 1423 I 31; Wilhelmus Erhardi (Gerardi) de Leydis 1418 II 25, 1420 I 20, 1421 I 7, 1421 I 26

Macedónia ehem. Kom. (Kom. Torontál, Ungarn, rumän. Macedonia, Rumänien) Macedonia

Ladislaus 1418 II 14

Màgerl

[Johannes] 1409 II 3

Mährisch-Ostrau (tschech. Moravská Ostrava, Mähren, Tschechien) Ostrauia Petrus Fabri 1428 I 2

Magdeburg (kreisfr. Stadt, Landeshauptstadt, Sachsen-Anhalt, Dtld.) Maydburg

Mathias Pernicz (Bernicz) (Saxo) 1416 I 25, 1417 I 9

Mayenschein

Wilibaldus 1437 I 8

Maiersch (Marktgem. Gars am Kamp, GB Horn, NÖ) Måyrzz

Johannes 1418 I 5, 1420 II 27, 1422 II 10

Mainz (Kr., RB Rheinhessen-Pfalz, Rheinland-Pfalz, Dtld., Erzstift) Maguntinensis, Maguntinus

Conradus Arnoldi 1430 I 20; Phylippus de Cronemberg 1414 I 2; Rabanus de Lybenstain 1430 I
16; Richardus de Superiori Lapide 1430 I 17; Theodricus de Staffel 1416 II 14

Mayr

Nicolaus 1421 I 12

Mayselstain, Mayslstain

Caspar 1405 II 2, 1408 I, 1409 II, 1414 I, 1416 I, 1419 II, 1424 I

Maior Glogouia s. Glogau

Maisenberg (mehrfach in Bayern, Dtld. oder Meisenberg Stadt Marsberg, Kr. Hochsauerlandkreis, RB Arnsberg, Nordrhein-Westfalen, Dtld. oder Meisenburg Stadt Mettmann, Kr., RB Düsseldorf, Nordrhein-Westfalen, Dtld.)

Maisemburg

Bernhardus Baustetter 1438 II 16

Maler $s$. Sartoris (Fridericus)

Manderscheid (Kr. Bernkastel-Wittlich, RB Trier, Rheinland-Pfalz, Dtld. oder Manderscheid Kr. Bittburg-Prüm, RB Trier, Rheinland-Pfalz, Dtld.) Manderscheyt Johannes 1417 I 1

Mankelwicz Paulus de Legnicz 1430 II 2 Marburg (Lahn) (Kr. Marburg-Biedenkopf, RB Gießen, Hessen) Marichpurk Johannes 1410 I 3

Marburg an der Drau (ehem. Land Steiermark, Stadt mit eigenem Statut, slowen. Maribor, Slowenien) Martpurga 
Bartolomeus 1426 II 8

Marcali (Kom. Somogy, Ungarn) Marcellis

Ladislaus 1419 I 8

Mardax

Stephanus 1429 I 8

Marichpurk s. Marburg (Lahn)

Marienthal (Ungarn oder Schlesien mehrfach) Vallis Sancte Marie

Petrus 1412 I 14

Marèuusch s. Moräutsch

Marienburg (ungar. Földvár, ehem. Kom.

Brássó, Ungarn, rumän. Feldioara,

Siebenbürgen, Rumänien) Mergenburg

Seruacius 1424 II 6

Martini

Nicolaus de Brůnsperg de Prussia

1436 I 3

Martpurga s. Marburg an der Drau

Mathie Goldperg (Goltperg)

Nicolaus de Wratislauia 1410 I 14, 1416 II 38

Mattsee (GB Salzburg, Sbg.) Maticensis

Thomas Matzseêr 1411 I 3

Matzseèr

Thomas 1411 I 3

Maulperger

Johannes de Altenmuldarff 1430 II 4

Maursteter

Jodocus de Campidona 1438 II 9

Mautern an der Donau (GB, NÖ) Mawttarn

Johannes 1420 II 15

Mawser

Andreas 1416 II 10

Mawttarn s. Mautern an der Donau

Meànsee s. Mondsee

Mèntelberger

Jodocus de Ynsprucka 1422 I 15

Mèrher

Thomas de Rastenueld 1419 II 8

Mährisch Neustadt (tschech. Uničov, Mäh-

ren, Tschechien) Noua Ciuitas

Gregorius Molitoris 1429 II 16;

Mathias Beschorn 1421 II 8;

Nicolaus 1431 II 9; Paulus Glen

1431 I 8; Vlricus Stamhawser

1424 I 14
Mêssemperger (illegitimer Sohn Herzog

Ernsts des Eisernen)

Ernestus 1421 II 11

Meck

Paulus de Ornbawr 1439 I 7

Medling s. Mödling

Megenhart

Heinricus de Blabüren 1424 I 4

Mehlsack (poln. Pieniężno, Ostpreußen, Polen) Melsak

Petrus Cerdonis 1420 II 16

Meilsdorfer (nö. Rittergeschlecht) Meylestorffer

Leonhardus 1418 II 1

Meyrs, Måyrzz s. Maiersch

Johannes 1418 I 5, 1420 II 27, 1422 II 10

Meyssilting (unbestimmt, wohl R)

Nicolaus Speyser 1409 I 9, 1422 I 4

Melchaymer

Georius de Prawnaw 1408 II 1

$\operatorname{Melk}(G B, N O ̈)$

Vrbanus 1413 II 12

Melsak (s. Mehlsack)

Memmingen (Kr., RB Schwaben, Bayern, Dtld.) Menning

Conradus Seybolt 1418 I 23; Johannes Vetter 1414 I 12; Vlricus Lager 1421 II 6

Mendorfer

Caspar 1402 I 5

Mendscenth s. Mindszent

Menkler

Wigandus de Hohemberg 1423 I 3

Menning s. Memmingen

Menseer

Bartholomeus 1423 I 7

Méra (Kom. Borsod-Abaúj-Zemplén, Ungarn oder ehem. Kom. Kolozs, Ungarn, rumän. Mera, Siebenbürgen Rumä-

nien) Mera

Barnabas 1431 I 10

Mergenburg s. Marienburg

Merswein

Johannes de Argentina 1417 II 4, 1418 II 4

Meschen (ungar. Muzsna, ehem. Kom. 
Nagy-Küküllö, Ungarn, rumän. Moşna,

Siebenbürgen, Rumänien) Můschna,

Muschen, Muschna

Antonius 1427 II 6; Petrus de Septemcastris 1434 I 4; Thomas de Septemcastris 1411 II 16, 1413 I 29

Messenpach s. Miesenbach

Michaelis

Andreas de Zerblin 1421 I 23; Andreas de Wratislauia 1425 II 5

Micheler

Nicolaus 1419 II 10

Miesenbach (viell. GB Wiener Neustadt,

$N O ̈)$ oder Miesenbach bei Birkfeld ( $G B$

Birkfeld, Stmk.)

Marquardus 1416 II 11

Miloslaw (poln. Mitostaw, Stadt südl. von

Wreschen, Bez. Posen, Polen) Milas-

lauia

Christoforus 1420 II 3

Miskolc (Stadt im Komitatsrang und

Verwaltungssitz des Kom. Borsod-

Abaúj-Zemplén, Ungarn) Myskolcz,

Myschkolcz

Andreas 1434 II 5, 1438 I 11

Miskowicz

Valentinus 1423 I 14

Mindszent (Kom. Csongrád, Ungarn oder Ungarn, Slowakei und Siebenbürgen mehrfach) Mendscenth

Valentinus 1433 I 10

Mittenwald (Kr. Garmisch-Partenkirchen, RB Oberbayern, Bayern, Dtld.) Mittenbald

Wilhelmus Steger 1429 II 26

Mödling (GB, NÖ) Medling Johannes Wingler 1432 II 6

Möttnig (ehem. Land Krain, Bez. Stein, slowen. Motnik, Slowenien) oder Möttling (ehem. Land Krain, Bez. Tschernembl, slowen. Metlika, Slowenien) Mồtnicz

Leonhardus Selgerêt 1414 II 4

Molhausen s. Mühlhausen (Thüringen)

Molinpach s. Mühlbach
Molitoris

Eberhardus de Nuremberga 1415 I 12; Gregorius de Noua Ciuitate 1429 II 16

Molnári (Kom. Zala, Ungarn) Mòlnar, Meoner

Dominicus 1424 I 11, 1428 I 13

Molzbichl (viell. Stadt Spittal an der

Drau, GB Spittal an der Drau, Ktn.)

Moltzpüchel

Erasmus Drüsselchind 1415 I 10

Monacum s. München

Mondsee (OÖ, Benediktinerkloster) Mềnsee

Vlricus 1420 II 6

Mons Petri s. Petersberg

Mons Sancti Georii Vallis Eni (unbestimmt, A)

Gregorius 1413 II 3

Mons Sancti Viti (unbestimmt)

Conradus Reysperger 1421 II 3

Montes Kutnae, Montes Chutnae s. Kuttenberg

Moosburg a. d. Isar (Kr. Freising, RB Oberbayern, Bayern, Dtld.) Mospurg Castulus Nůsser 1438 II 3

Moräutsch (ehem. GB Egg, Krain, slowen. Moravče, Slowenien) Mareausch

Petrus Polcz 1417 I 19

Moschicz

Michel 1420 II 9

Mühlbach (ungar. Szászebes, rumän.

Sebeş, Siebenbürgen, Rumänien) Mùl-

bach, Müllenbach, Mưlnpach, Mu-

lenbach, Mulembach, Molinpach

Antonius (de Septemcastris) 1422 I

21, 1425 II 4, 1427 II 12, 1428 I

11; Cristannus 1432 II 4; Valentinus in Septemcastris 1437 II 11

Mübldorf a. Inn (Kr., RB Oberbayern, Bayern, Dtld.) Müldorf, Altenmuldarff

Heinricus 1407 II 4; Johannes Maulperger 1430 II 4

Münheym

Johannes de Praga 1428 I 1 
München (kreisfr. Stadt, RB Oberbayern, Bayern, Dtld.)

Chonradus Zeydlarear 1410 II 2; Johannes Giesser 1440 II 6; Johannes Heller 1437 I 15; Johannes Stainger 1431 I 9; Leonhardus Tenniger 1429 II 22; Ludouicus Leupolt 1442 I 15; Vlricus Horlactawer 1411 II 1; Wilhelmus Grayspach 1428 II 6

Münsterberg in Schlesien (poln. Ziębice, Niederschlesien, Polen) Münsterberg Johannes de Schram 1437 II 10 Mürbacher

Petrus de Lincz 1426 II 2

Mưrlein

Georius 1415 I 17
Mưyther Johannes 1411 II 6

Můschna, Muschen, Muschna s. Meschen Mühlhausen (Thüringen) (Kr. UnstrutHainich-Kreis, Thüringen, Dtld.) Molhausen Gregorius Andree 1442 I 7

Mulhaimer Johannes 1434 I 6

Mulpacher Cristannus de Kufstain 1426 I 6 Murher

Cristannus de Grassertal 1412 I 5 Musson (Belgien) (viell.) Mưsson Jacobus Johannis 1422 I 5
Nabburg (Kr. Schwandorf, RB Oberpfalz, Bayern, Dtld.) Napurga

Heinricus 1421 II 20

Nabok, Nobag, Nowag

Petrus de Nissa 1420 II 18, 1423 I 30, 1428 I 10, 1428 I 12, 1432 II

Nafczer, Naffczer

Petrus de Wysenstaig (Wisenstaig) 1438 II 4, 1440 II 5, 1441 I 12

Nappersdorf-Kammersdorf (GB Holla-

brunn, NÖ) Napelstorff

Michel 1421 I 10

Napurga s. Nabburg

Nas

Andreas 1412 II 13

Nebraw

Bernhardus 1414 I 25

Neczowencz

Ambrosius de Golaw 1417 I 16

Neisse (poln. Nysa, Oberschlesien, Polen)

Nissa, Nyssa

Caspar Fridwalt 1416 I 23; Jacobus Bawdewek 1416 II 26; Jacobus Pistoris 1416 I 5; Johannes Preylant 1416 I 6; Nicolai 1431 II; Paulus Syndrami 1420 II 17,
1424 II 11; Petrus Nabok (Nowag) 1420 II 18, 1423 I 30; Nicolaus Peck 1424 I 9

Neograd (ungar. Nógrád, Komitat, Ungarn, oder Nógrád, Kom. Nógrád, Ungarn) Newgradiensis Johannes 1427 II 2

Nernshaymer Vlricus de Haydenhaym 1415 II 6 Neuburg (unbestimmt) Newburga, Newmburga

Dyonisius 1426 II 4; Jacobus Rosenczweig 1436 II 5; Vlricus Gewalt 1421 II 12

Neuburgensis, Neunburgensis s. Klosterneuburg

Neumann

Stephanus de Freinstat 1430 II 9

Neumarkt (Neumarkt i. d. Opf., Kr., RB Oberpfalz, Bayern, Dtld. oder Neumarkt-Sankt-Veit, Kr. Mühldorf a. Inn, RB Oberbayern, Bayern, Dtld. oder Neumarkt am Wallersee, GB Neumarkt bei Salzburg, Sbg.) Nouum Forum Jodocus Hawsner 1437 I 14; Jo- 
hannes Aichelperger 1429 II 5, 1432 I 18

Neumarkt (poln. Środa Ślaska, Niederschlesien, Polen) Nouum Forum Nicolaus de Seydlicz 1411 II 5

Neumarkt (wahrscheinl. Neumarkt in Steiermark, GB, Stmk.) Nouum Forum prope Frisacum

Johannes (Czopot) 1429 I 5, 1431 II 22

Neustadt (ungar. Balmazújváros, Kom. Hajdú, Ungarn oder ungar. Keresztényfalva, ehem. Kom. Brássó, rumän. Cristian, Rumänien oder ungar. Nagybánya, ehem. Kom. Szatmár, rumän. Baia Mare, Rumänien oder ungar. Ujváros, ehem.Kom. Nagy-Küküllö, rumän. Noisțtat, Rumänien oder Neustadt an der Waag, ungar. Vágújhely, ehem. Kom. Nyitra, slowak. Nové Mesto nad Váhom, Slowakei) Nova Civitas Johannes Lewbner 1420 I 6

Newburga, Newmburga s. Neuburg Newgradiensis s. Neograd

Newmburga Claustralis, Newmburgensis, Newnburga, Newnburgensis $s$. Klosterneuburg

Newsidler Petrus de Laa 1426 II 6, 1432 II 12

Newsteter Johannes 1416 II 27

Nicolai Johannes de Brunna 1412 II 7

Nidda (Kr. Wetteraukreis, RB Darmstadt, Hessen, Dtld.) Nydden Johannes 1410 II 5

Niederaltaich (Kr. Deggendorf, RB Niederbayern, Bayern, Dtld., Benediktinerkloster) Nidernaltach, Nydernaltach, Altach, Inferior Altach Conradus de Salczburga 1411 II 2, Gothardus 1413 II 10; Heinri- cus 1412 II 8; Symon 1436 II 4, 1442 I 9; Thomas Do̊rndel 1413 II 17

Nikolsburg (tschech. Mikulov, Mähren, Tschechien) Nicolspurga Johannes Amelprecht 1417 II 10

Nissa, Nyssa s. Neisse

Nobag $s$. Nabok

Nördlingen (Kr. Donau-Ries, RB Schwaben, Bayern, Dtld.) Noordlinga, Noorlinga, Norrlingen Franciscus Althaymer 1423 II 8;

Franciscus Rewtter 1439 II 1; Johannes Ortlini 1440 II 1

Nóráp (Kom. Veszprém, Ungarn) Norap Thomas 1431 II 11

Nordhausen (Kr., Thüringen, Dtld.) Northusen

Henricus Slick 1432 I 11

Nosticz

Heinricus 1421 I 25

Noua Ciuitas s. Mährisch Neustadt

Nova Civitas s. Neustadt

Nouum Forum s. Neumarkt

Nowag s. Nobag

Nürnberg (Kr., RB Oberfranken, Bayern, Dtld.) Nuremberga, Nurmberga, Nưrmberga Eberhardus Molitoris 1415 I 12; Heinricus Pawr 1441 I 3; Heinricus Tandorffer 1413 II 9, 1417 II 1, 1418 II 5, 1419 I 17; Johannes Pusmer 1430 I 15

Egidienkirche Sanctus Egidius de Nürmberga

Erhardus 1437 II 12

Nüsser

Castulus de Mospurg 1438 II 3

Nůsdorffer

Vdalricus 1436 II 2

Nuremberga s. Nürnberg

Nurmberga. s. Nürnberg 
Obergurk (slowen. Krka, ehem. Land Krain, Bez. Littai, heute Gem. Grosuplje, Slowenien) oder Oberburg (slowen. Gornji Grad, ehem. Land Steiermark, Bez. Cilli, slowen. Gornji Grad, Slowenien) Obernburg, Obernburk, Oberngurkch

Laurencius 1431 I 7, 1433 II 13, 1436 II 10

Obernburg (wohl Obernburg a. Main, Kr. Miltenberg, RB Unterfranken, Bayern, Dtld.) Oberberga

Conradus Reintaler 1426 II 10

Oberstein (Bayern, Dtld. mehrfach) Superior Lapis

Oder

Richardus 1430 I 17

Hilprandus de Dorffen 1429 II 10

O̊berlinga s. Überlingen

O̊ch

Jacobus de Pappenhaim 1424 I 7

O̊denfurter

Petrus de Liechtenburg 1431 I 4

O̊czing s. Ötzing, Ötzingen

O̊der

Thomas de Achspach 1419 I 6, 1422 I 2, 1426 I 9, 1426 II

Öhringen (Kr. Hohenlohekreis, RB

Stuttgart, Baden-Württemberg, Dtld.)

O̊ringèw, O̊ringew, Orengew

Vlricus (Sunperger) 1430 II 3, 1434 II 10, 1438 I 15

Oels (viell. tschech. Olešnice, GB Kunštát,

Mähren, Tschechien) Olsna

Johannes Nicolai Fabri 1429 II 19;

Nicolaus Reyman 1429 II 18

Ötting (Alt) (Kr., RB Oberbayern, Bay-

ern, Dtld. oder (Neu)Ötting, Kr. Al-

tötting, RB Oberbayern, Bayern, Dtld. oder Oettingen i. Bay., Kr. Donau-

Ries, RB Schwaben, Bayern, Dtld.)

O̊tting, O̊ttinga, O̊ttingen;

O̊tinga

Franciscus Granatoris 1410 II 1; Fridericus Stawthaimer 1407 II 3;
Johannes Stawdhaymer 1430 II 8;

Vlricus Eysemman 1424 I 8

Oettingen (Grafengeschlecht im Riesgau)

O̊tingen

Albertus 1432 II 1; Vlricus 1429 II 1

Ötzing (Gem. Tiefenbach Kr. Passau, Kr.

Passau (Land), RB Niederbayern,

Bayern, Dtld. oder Ötzingen, Kr. Westerwaldkreis, RB Koblenz, Rheinland-

Pfalz, Dtld.) O̊czing

Georius Mưrlein 1415 I 17

Olderspach s. Aldersbach

Olmütz (tschech. Olomouc, Mähren, Tschechien) Olmutz, Olmuncz, Olmüncz,

Olomutz, Olomucz, Olomucensis, Olomuncensis, Olomoncensis, Olmoncensis

Andreas 1412 II 6; Jeronimus (Lantuoyt) 1428 I 6, 1430 I 33,

1433 II 14; Jeronimus 1429 II 8;

Jeronimus Vogelsang (Vogelsangk)

1437 I 19, 1439 II 3, Johannes

1416 II 5; Johannes Hers (Håz, Has) (de Brunna) 1429 I 1 ,

1432 I 20, 1438 I 14; Johannes

Pawswågl 1441 II 3; Nicolaus

Gramus 1422 II 1; Nicolaus

Schoonperger de 1428 I 7, 1431 II

21; Symon Alberti 1413 I 3

Olsna s. Oels

Omlaschz s. Hamlesch

Onalbach s. Ansbach

Opauia s. Troppau

Oppeln (poln. Opole, Oberschlesien, Polen) Opoliensis

Heinricus de Campa 1430 I 22

Orengew, Oringew s. Öhringen

Ornbawr (wahrscheinl. verschrieben aus Hembawrs. Hemau)

Ortenberg (entweder Ortenberg Ortenaukreis, Kr. Ortenaukreis, RB Freiburg $i$. Br., Baden-Württemberg, Dtld. oder Ortenberg Wetteraukreis, Kr. Wetteraukreis, RB Darmstadt, Hessen, Dtld.) Johannes Quentin 1433 I 4 
Ortenburg (niederbaier. Grafengeschlecht) Artenberg, Artemberg Oswaldus 1421 I 1; Vlricus 1418 II 9

Ortlini

Johannes de Nơrlinga. 1440 II 1

Osterhofen (Kr. Deggendorf, Niederbayern, Bayern, Dtld.) Astershofen, Osterhofen

Andreas 1413 I 7; Wenceslaus 1418 II 11
Osterode i. Ostpr. (poln. Ostróda, OstpreuBen, Polen) Osterror Johannes Turinge de Prawnsperg 1413 I 24

Ostrauia s. Mährisch-Ostrau

Ottmachau (poln. Otmuchów, Oberschlesien, Polen) Othmuchouiensis Nicolaus Wenke 1422 II 2, 1423 II 9 Oyta s. Friesoythe

Pachenstain

Conradus 1429 II 15

Pachmůlne̊r, Pachmůlner, Pachmulnể,

Pachmulner, Pachmúlner

Petrus (de Eberspewnt) 1419 I 21, 1422 I 3, 1424 I 22, 1424 II, 1430 I 25, 1431 I, 1434 II, 1438 I, 1441 II

Paderborn (Kr., RB Detmold, Nordrhein-

Westfalen, Dtld.) Paderburnensis,

Palborn

Hermannus Deys 1417 II 14; Johannes 1423 II 10

Påmburg s. Baumburg

Pểrtl (Bẻrtel)

Johannes de Salczbach (Sulczpach) 1438 II 5, 1441 I 11

Pềrl

Paulus (Pa[u]lus) de Wienna 1409 II 11, 1419 II 19, 1419 II 20, 1420 II, 1420 II 22, 1425 II, 1428 II, 1431 II

Payer, Payr

Wolfgangus de Hederstorff 1421 I 9, 1430 I 26

Palborn s. Paderborn

Palocz s. Pavlovce nad Uhom

Pamenczina (unbestimmt, $H$ )

Petrus 1418 II 23

Pankota (ungar. Pankota, ehem. Kom. Arad, Ungarn, rumän.Pâncota, Banat, Rumänien) Pankata, Pankotha

Johannes 1441 I 10, Johannes Zimischem 1413 I 25

Pappenheim (Kr. Weißenburg-Gunzhausen, RB Mittelfranken, Bayern, Dtld.) Papenheim, Pappenhaim

Jacobus O̊ch 1424 I 7; Johannes Spềt 1412 II 11, 1420 I 18; Vlricus Werder 1413 II 16

Pappenheim (mittelfränk. Adelsgeschlecht)

Poppenhaim, Poppenhaim

Conradus 1413 I 32, Georius 1413 I 31

Parkstainer

Emeramus de Weyden 1439 I 9

Parrewtter

Johannes 1433 II 4

Paskowicz

Nicolaus 1426 II 5

Passau (Kr., RB Niederbayern, Bayern,

Dtld.) Patauia, Patavia, Patauiensis,

Pataviensis

Caspar Czener 1431 I 6, 1434 II 11; Erhardus Herrant (de Newmburga Claustrali) 1422 I 20, 1425 II 3, 1430 I 30, 1430 II, 1432 I 15, 1433 II; Fridricus Kraft (Krafft) 1406 II 15, 1417 II 6, 1417 II 7, 1418 I, 1418 I 3; Georius de Battaw 1415 II 13; Georius Jågenrewảtter (Jågenreiter, Jegenrewtter) 1428 II 7, 1431 I 12, 1433 II 15; Hartungus de Cappel 1432 I 7, 1433 I; Johan- 
nes Chlosner 1419 I 7; Leonardus Layminger 1407 II 6; Marcus Enenkel de Albrechtzperg 1416 I 17; Marquardus de Messenpach 1416 II 11; Nicolaus 1413 I 33;

Petrus Chotter de Patauia 1416 II 12; Siluester Wager 1416 I 14; Theobaldus Türndl 1438 II 10; Vlricus de Artemberg 1418 II 9; Vdalricus Nůsdorffer 1436 II 2; Vlricus Reysperger 1419 I 13

Pauli

Petrus de Alba Regali 1416 I 20

Pavlovce nad Uhom (ungar. Pálóc, ehem. Kom. Ung, Slowakei) Palocz Petrus 1431 II 2

Pawmburg, Pawburgensis s. Baumburg Pawr

Heinricus de Nůrmberga 1441 I 3

Pawswägl

Johannes de Olomutz 1441 II 3

Pechstal s. Pöggstall

Peck

Nicolaus de Nyssa 1424 I 9

Pemeshaim s. Bensheim

Pencznalber

Johannes 1438 II 8

Peraewt s. Bayreuth

Perching s. Berching

Perchtoldsdorf (GB Mödling, NÖ)

Perchtoltzdorff, Berchtoltstorf

Andreas Feynockh 1432 I 8; Johannes 1406 II 2, 1421 II 1, 1422 I

Pernicz, Bernicz

Mathias (de Maydburg) (Saxo) 1416 I 25, 1417 I 9

Pernstayn, Pernstaìn s. Bernstein

Perold

Nicolaus 1403 I 2, 1411 II 18

Petersberg (Petersburg) (ungar. Barcaszentpéter, ehem. Kom. Brássó, Ungarn, rumän. Sânpertru, Rumänien) Mons

Petri

Martinus Helman 1416 II 29

Petirbicz

Martinus 1412 I 10
Petri

Georius de Briswicz 1425 I 2

Pettau (ehem. Land Steiermark, Stadt mit eigenem Statut, slowen. Ptuj, Slowenien) Petouia

Petrus Hümel 1439 I 2

Pfaffendorff (Familienname)

Andreas 1417 I 6

Pflancz

Johannes 1423 I 21

Phêffel

Michel de Ingolstat 1418 II 24

Phaffenhofer

Martinus de Sancto Floriano 1427 II 7

Pherlini

Petrus 1409 II 2

Phister

Stephanus de Steyer 1419 II 14

Phờrtner

Conradus de Jukarim 1412 I 6

Piburga s. Biburg

Piderman

Cristoforus de Wratislauia 1437 I 2

Pillichsdorf (GB Wolkersdorf, NÖ) Pi-

lichtorf

Thomas de Creyuelt 1432 I 14

Pirchachhausen $s$. Birchachhusen

Pistoris

Jacobus de Nissa 1416 I 5

Pleusnicz s. Pleisnitz

Pleyntinger

Johannes Pleyntinger 1417 I 11

Pleisnitz (Pleissnitz) (wohl ungar. Pelsöc,

ehem. Kom. Gömör és Kishont, slowak.

Plešivec, Slowakei) Pleusnicz

Nicolaus Kitzka (Kyczka, Kitschka) 1411 II 17, 1413 I 30, 1419 II 15

Plesner

Hainricus de Eschenbach 1430 I 14

Plo̊ntinger

Georius 1429 II 7

Plossenstauffen (unbestimmt, $R$ )

Johannes Stauffer 1438 II 17

Pluemenekcher, Plůmenegker

Johannes de Argentina 1434 I 14, 1436 II 15,1439 II 10 
Podatzschin s. Bochdaschin

Pöggstall (GB Melk, NÖ) Pechstal

Mathias Schrat 1430 I 21

Pölla (NÖ mehrfach) oder Pöllau (Stmk.

mehrfach) Pòlan, Aldenpolan

Johannes Stark, 1412 II 18; Martinus 1442 I 3

Pòmer

Conradus de Eystauia 1437 I 16

Polcz

Petrus 1417 I 19

Polzmacher, Polczmacher, Poltzmacher Johannes (de Brunna, Brünna) 1428 I

4, 1430 I 34, 1432 II 8, 1434 II 13, 1436 I, 1439 I, 1441 I

Poppenhaim s. Pappenheim (Adelsname)

Póráz (ungar., ehem. Kom. Zólyom, Ungarn, slowak. Povraznik, Slowakei oder ungar. Vereshegy, ehem. Kom. Szepes, slowak. Porác, Slowakei oder ungar. Boróc, ehem. Kom. Bács-Bodrog, serb. Obrovac, Serbien) Worach

Benedictus 1418 I 7

Poschega (kroat. Slavonska Požega, Slawonien, Kroatien) Poseda

Petrus 1439 I 4

Poslowitz (wohl tschech. Pozlovice, GB

Bojkovice, Mähren, Tschechien) Wos-

lawicz

Nicolaus Jenkebicz 1429 I 4

Posser

Jeronimus (de Salczburga) 1436 II 3, 1439 II 4, 1442 I 10

Possilwicz s. Boskowitz

Poter

Johannes Sartoris de Fridwerkch 1429 I 15

von Pottendorf (österr. Herrengeschlecht)

Potendorff

Albertus 1419 II 12

Poueda

Johannes 1416 I 10

Prag (Praha, Böhmen, Tschechien) Praga,

Pragensis

Johannes Münheym 1428 I 1; Michel de Rèdisch 1424 II 9; Nicolaus Heinrici 1424 II 8
Prannt

Vlricus 1440 I 1

Pratesser

Martinus 1430 II 18

Prawnaw s. Braunau am Inn

Prawnsperg s. Braunsberg

Preinberg s. Brennberg

Preller

Johannes 1423 II 2

Prenner

Fredericus 1414 I 27, 1414 I 28, 1414 II 16

Prepticz

Heinricus de Slesia 1427 I 6

Pressater

Wilhelmus de Amberg 1413 I 18

Preßburg (ungar. Pozsony, slowak. Bratislava, Slowakei) Bosnoniensis

Albertus de Borzislauicze 1414 I 10

Preußen Prussia, Prusia, Pruscia

Andreas Langkhaim 1428 II 2;

Franciscus Resil (Resel, Rossel) 1414 I 20, 1416 I 32, 1416 II 37, 1416 II 41; Hermannus Huxer 1430 II 1; Nicolaus 1409 II 1; Nicolaus Martini de Brůnsperg 1436 I 3; Petrus Warnaw (Barna) 1414 I 19, 1416 I 31, 1416 II 36, 1416 II 40

Preylant

Johannes de Nissa 1416 I 6

Preym

Johannes de Cliuis 1416 I 7

Preyzyger

Wilhelmus 1402 I 1

Priwitz (Pritz) (ungar. Privigye, ehem.

Kom. Nyitra, slowak. Previdza, Slowa-

kei) Priuidia

Michael 1430 II 11, 1434 I 17, 1435 II 17

Prost

Leonardus 1407 II 1

Prüm (Kr. Bitburg-Prüm, RB Trier, Rheinland-Pfalz, Dtld.) Průmea

Nicolaus 1413 II 7

Prün s. Brunn

Prugk s. Bruck an der Mur 
Pruglegker

Conradus de Welsa 1440 II 8

Prunger

Johannes de Frankfordia (Franckfordia) 1417 II 8, 1418 I 2, 1419 II 17,1419 II 16

Průmea s. Prüm

Pruscia, Prusia, Prussia s. Preußen

Pruszella s. Bruchsal

Puch (Bayern, Dtld. mehrfach) Puech

Cristannus Håppel 1420 I 1

Pudlein (ungar. Podolin, ehem. Kom.

Szepes, slowak. Podolinec, Slowakei)

Pudlinum
Johannes 1427 I 1, 1442 I 13

Puech s. Puch

Purkhawsen, Purchhawsen, Purkhusa,

Burghusen s. Burghausen

Purger

Fridericus de Helpach 1419 I 20

Purgfinger

Stephanus 1402 I 7

Pusmer

Johannes de Nurmberga 1430 I 15

Putnyt

Andreas de Aw 1418 I 11
Quedenow

Jodocus de Künigsperg 1416 I 19

Quentin

Johannes de Ortenberg 1433 I 4
Quinque Ecclesiae, Quinqueeclesiae,

Quinqueecclesiensis s. Fünfkirchen
Rademan

Johannes de Lukau 1430 I 5, 1434 I 18

Radostin oder Radostitz (Tschechien mehrfach) Radhost

Hodiko 1417 II 11

Råbel

Johannes de Corona 1424 II 7 , 1427 II 13

Rẽdisch s. Aug-Radisch

Rẻntel

Wilhelmus de Awsse 1415 I 18

Råtelchouer

Johannes 1430 II 17

Rätsch (Retsch) (ungar. Szebenrécse, ehem.

Kom. Szeben, rumän. Reciu, Sieben-

bürgen, Rumänien) Recz, Redsch

Andreas 1430 II 20, 1433 II 7

Råwching, Rewèhing, Rủhing Ruyhing

Conradus (de Freyburg) 1411 II 20, 1416 I 30, 1416 II 35, 1417 I
Rèwtemberger

Johannes 1420 II 1

Rain (mehrfach in Dtld.)

Cristoforus Guldein 1423 I 24, 1426 II 19

Rayner

Jacobus 1434 I 2

Raptoris

Johannes 1432 I 13

Rastaker

Leonardus de Villaco 1421 I 15

Rastenfeld (GB Krems an der Donau, NÖ)

Rastenueld

Thomas Mearher 1419 II 8

Rathsambausen (elsäss. niederadelige Familie, benannt nach Rathsamhausen, 4 km östl. von Schlettstatt, franz. Sélestat, Dep. Bas-Rhin, Frankreich) Raczenhausen, Raczenhawsen

Bernhardus 1412 I 22; Johannes 1421 I 14 
Ratibor (poln. Racibórz, Oberschlesien, Polen) Rathibornensis

Johannes 1438 II 11

Ratispona, Ratisponensis, Ratispanensis s. Regensburg

Rattenberg (GB, T oder Kr. Straubung-Bogen, RB Niederbayern, Bayern, Dtld.)

Rotemberga

Fridericus Grenn 1416 II 9

Ravensburg (Kr., RB Tübingen, BadenWürttemberg, Dtld.) Rauenspurg

Petrus 1432 I 12, 1434 I 15

Rawch

Heinricus de Tagersheim 1417 I 17, 1421 I 16

Rebiczer

Nicolaus 1432 I 9

Rédics (Kom. Zala, Ungarn) Redisch Johannes 1431 II 14

Redler

Johannes 1413 II 14

Regelshofer, Regelshoffer, Regelzhofer

Mathias (de Herriden) 1408 I 3, 1410 I 18, 1413 II 6, 1419 I

Regensburg (Kr., RB Oberpfalz, Bayern, Dtld.) Ratispona, Ratisponensis, Ratispanensis

Conradus Reykershouer (Reykershofer, Reikershofer, Reickershofer) 1430 II 22, 1434 I 19, 1435 I 11, 1435 II 11; Cristannus de Friberg 1440 II 4; Erhardus Satelboger 1414 II 5; Hinricus Kranpergher 1403 I 1; Heinricus Streytperger 1422 I 6; Johannes Augustini 1414 II 13; Johannes Awer 1416 I 24; Johannes Gnendorffer 1422 I 13; Johannes Streyperger 1403 I 5; Leo Geysenuelder 1420 II 19; Leonardus Prost 1407 II 1; Liebhardus 1416 II 32; Martinus 1416 II 33; Nicolaus Rebiczer 1432 I 9;

Oswaldus Strawss 1419 I 5; Vlricus comes de Artemberg 1418 II 9; Vdalricus Nůsdorffer 1436 II 2; Vlricus Werthart 1421 II 2

Regerteln (poln. Rogiedle, ehem. Landkreis
Heolsberg, poln. Lidzbark-Warminski,

Ostpreußen, Polen) Rogettel

Nicolaus 1409 II 5

Rehden (wohl poln. Radzyń Chetminski,

Westpreu(en, Polen) Reddin, Reddi-

num

Cristamus 1420 II 8; Nicolaus Cristanni 1420 I 2

Reiberstorffer

Nicolaus de Montibus Chutnis 1412 II 9

Reychel

Nicolaus de Wratislauia 1433 II 1

Reichersberg (GB Obernberg am Inn, OÖ)

Reichersperg

Erasmus 1437 I 1, 1440 I 7

Reykershouer, Reykershofer, Reikersho-

fer, Reickershofer

Conradus 1430 II 22, 1434 I 19, 1435 I 11,1435 II 11,1435 II 15

Reyman

Nicolaus de Olsna 1429 II 18

Reyndel

Conradus de Fredeberg 1422 II 9

Reintaler

Conradus de Oberberga 1426 II 10

Reyntaler

Erasmus 1408 I 7

Reynwalt

Heinricus 1437 I 7

Reisbach (Kr. Dingolfing-Landau, RB Niederbayern, Bayern, Dtld.) Reyspach,

Reysspach

Johannes Reysegk 1434 II 4; Petrus

Hekch 1423 I 16

Reisberg, von (sbg. Ministerialengeschlecht)

Reysperger

Conradus 1421 II 3; Vlricus 1419 I 13

Reysegk

Johannes de Reysspach 1434 II 4

Reysperger s. Reisberg, von

Resch

Johannes (de Swabach) 1428 II 1, 1433 II 10

Reschel

Fredericus 1423 I 32 
Resil, Resel, Rossel

Franciscus de Prusia 1414 I 20, 1416 I 32; 1416 II 37; 1416 II 41

Resinkirche, Rosenkirch (unbestimmt, $H$ )

Michel (Jeskonis) 1411 I 1, 1413 I 19

Reussen (ungar. Rüsz, ehem. Kom. Szeben, rumän. Ruşi, Siebenbürgen, Rumänien) Russia

Petrus Sculteti 1423 I 6

Reutlingen (Kr., RB Tübingen, BadenWürttemberg, Dtld.) Rütlinga, Rudlinga

Conradus Seczel 1416 II 25; Georius 1415 I 20; Jacobus Tols 1429 II 2; Nicolaus Weinschenk 1418 II 2

Rewtter

Franciscus de No̊rlingen 1439 II 1 Rex

Johannes 1409 II 6

Riedlingen (Stadtteil von Donauwörth, $K r$.

Donau-Ries, RB Schwaben, Bayern, Dtld. oder Kr. Biberach, RB Tübingen, Baden-Württemberg, Dtld. oder Stadtteil von Kandern, Kr. Lörrach, RB Freiburg, Baden-Württemberg, Dtld.) Rudlinga

Georius 1415 I 20; Jacobus Tols 1429 II 2; Nicolaus Weinschenk 1418 II 2

Riedrer

Conradus 1437 II 3

Riga (Hauptstadt von Lettland)

Johannes Loman 1418 I 13

Ringelstein (viell. Stadt Büren, Kr. Paderborn, RB Detmold, Nordrhein-Westfalen, Dtld.) Ringenstain Johannes 1442 I 6

Ro̊chel

Johannes 1408 I 1

Röchel

Martinus 1411 I 12

Ròtlini

Johannes de Opauia 1428 I 5 (viell. identisch mit Johannes Sews; siehe dort)
Rogettel s. Regerteln

Roggenburg (Kr. Neu-Ulm, RB Schwaben, Bayern, Dtld.) Roggenburgensis Johannes (de Rotemberg) 1423 I 10, 1426 I 12

Roll

Heinricus 1414 II 12

Roraw

Heinricus 1427 I 7

Rosemberg

Heinricus 1416 II 28

Rosenau (GB Zwettl, NÖ) Rosenaw

Johannes 1421 I 11

Rosenau (ungar. Rozsnyó, ehem. Kom.

Gömor és Kishont, Ungarn, slowak.

Rožñava, Slowakei oder ungar. Barcarozsnyó, ehem. Kom. Brássó, rumän.

Râşnov, Siebenbürgen, Rumänien oder

Rožnau am Radhost, tschech. Rožnov pod Radhoštěm, Mähren, Tschechien oder Schlesien mehrfach) Rosenaw Jacobus 1430 I 9

Rosenczweig Jacobus 1436 II 5

Rosenczwey Jacobus de Weyssemburga 1442 I 11

Rosenheim (Oberbayern, Kr., RB Oberbayern, Bayern, Dtld.)

Martinus Wanislant 1416 II 17

Rosenkirch $s$. Resinkirche

Rossel $s$. Resil

Rot (Baden-Württemberg, Dtld. mehrfach oder Roth, Dtld. mehrfach)

Jodocus (Judocus) (de O̊berlinga, Vberling) 1408 I 5, 1420 I 5, 1431 II 20

Rota

Laurencius 1418 I 18

Rotemberg (Verschreibung aus Roggenburg, s. dort)

Rotenberg (Stadtteil von Stuttgart, RB, Baden-Württemberg, Dtld. oder Stadtteil von Rauenberg, Kr. Rhein-Neckarkreis, RB Karlsruhe, Baden-Württemberg, $D$ tld. oder Rothenberg mehrfach in Bayern und Hessen, Dtld.) Rotemberga Fridericus Grenn 1416 II 9 
Rotemberga s. Rattenberg oder Rottenberg Rothenburg (Oder) (wohl poln.

Czerwiensk, Niederschlesien, Polen)

Rotemburg

Cristoforus 1438 I 2

Roter

Heinricus 1419 I 16

Rothau (Markt Tittling, Kr. Passau

(Land), RB Niederbayern, Bayern,

Dtld.) Battaw

Georius 1415 II 13

Rothberg (ungar. Veresmart, ehem. Kom.

Szeben, Ungarn, rumän. Roşia, Sie-

benbürgen, Rumänien) Ruffus Mons

Leonardus 1402 I 10

Rottenberg (Markt Hosbach, Kr. Aschaffenburg (Land), RB Unterfranken,

Bayern, Dtld.) Rotemberga

Fridericus Grenn 1416 II 9

Rozgony (ehem. Kom. Abaúj-Torna, Ungarn, slowak. Rozhanovce, Slowakei)

Rozgon

Oswaldus 1437 I 3; Symon 1413 I 4

Rubennaw

Erasmus de Gribswaldis 1418 II 20

Ruch

Vlricus 1429 I 17

Ruckendorf (nö. Rittergeschlecht) Rukhen- dorf

Lampertus 1441 II 1

Rudlinga s. Riedlingen, Rüdlingen oder

Reutlingen

Georius 1415 I 20; Jacobus Tols 1429 II 2; Nicolaus Weinschenk 1418 II 2

Rüdlingen (Kanton Schaffhausen, Schweiz)

Rudlinga

Georius 1415 I 20; Jacobus Tols 1429 II 2; Nicolaus Weinschenk 1418 II 2

Rủckpass (unbestimmt, $H$ )

Thomas Sybart 1437 II 9

Conradus 1416 II 35

Rủhing, Ruyhing s. Råwching

Rül, R'uel

Johannes de Francfordia (Franckfordia, Frankfordia) 1435 I 3, 1438 I 13, 1441 II 5

Růsdarf (unbestimmt)

Nicolaus 1409 II 9

Rütlinga s. Reutlingen

Ruffus Mons s. Rothberg

Ruschlini

Johannes alias Judicis de Gewicz 1416 I 16

Russia s. Reussen

\section{$-S-$}

Sachs

Wolfgangus de Almekch prope Lambach 1434 I 13; 1437 II 14

Sèld s. Seld

Sagrabiensis s. Agram

Salczbach s. Sulzbach

Salczmesser

Nicolaus de Frankfordia circa Oderam 1413 II 18

Salina s. Hallein

Samland (Halbinsel zwischen dem Frischen und dem Kurischen Haff, Ostpreußen)

Sambiensis

Andreas Pfaffendorff 1417 I 6

Sana s. Zsana
Salzburg (Stadt und Hochstift; österr. Bundesland) Salczburga, Salczpurga, Salzburga, Salczburgensis, Salzburgensis

Clemens Almår 1415 I 8; Conradus 1411 II 2; Erasmus Freyberger 1422 II 6; Virgilius Albner 1420 II 20; Fredericus 1414 II 11; Jeronimus Posser 1436 II 3, 1439 II 4, 1442 I 10; Leonardus Scho̊ntrit 1438 II 2; Oswaldus de Artenberg 1421 I 1

Sanctus Egidus de Nưrmberga s. Nürnberg Sankt Veit bei Laibach (ehem. Land Krain, slowen. Šentvid, Stadtgemeinde Ljubljana, Slowenien) Sanctus Vitus prope Laybacum 
Johannes Czepeck 1413 I 13, 1431 II 25

Sanctus Vitus (unbestimmt)

Jacobus 1414 I 23

Sankt Florian (GB Linz-Land, OÖ) Sanctus Florianus

Florianus Im Wald 1435 II 8; Martinus Phaffenhofer 1427 II 7; Wolfgangus Kerspek 1418 I 12, 1421 II 16, 1423 I 25

Sankt Georgen am Ybbsfelde (GB Amstetten, NÖ) Sanctus Georius super Ybsueld

Petrus 1434 I 12

Sanctus Germanus (unbestimmt) Waltherus Schơrling 1412 I 18

Sankt Johann (Gem. Grafenwörth, GB Kirchberg am Wagram, NÖ) Sanctus Johannes prope Grauenwerd Johannes de Rosenaw 1421 I 11

Sanctum Ypolitum, Sanctum Yppolitum s. Sankt Pölten

Sankt Lambrecht (GB Neumarkt in Steiermark, Stmk., Ö) Sanctus Lampertus Johannes Schachner 1441 I 8

Sankt Leonhard am Forst (GB Mank, NÖ) s. Leonardus in Foresta Thomas Angelpekh 121433 I 3

Sankt Marein (Stadtgem. Wolfsberg, GB, Ktn.) oder Maria Rojach (Stadtgem. St. Andrä, GB Wolfsberg, Ktn.) Sancta Maria Vallis Lauentine Johannes Stro̊mberger 1438 II 7

Sankt Niklas an der Drau (Stadtgem. Villach, Stadt mit eigenem Statut, Ktn.) s. Nicolaus prope Villacum Vlricus Reysperger 1419 I 13

Sankt Peter ob Judenburg (GB Judenburg, Stmk., Ö) Sanctus Petrus prope Judenburgam Petrus 1402 I 9

s. Petrus in Karinthia (unbestimmt, Ktn.) Johannes Kẻser 1440 II 3

Sankt Pölten (Landeshauptstadt, NÖ) Andreas 1416 II 4; Caspar 1432 I 2, 1435 I 9, 1437 II 18, 1437 II
21; Michahel 1408 II 2; Petrus 1420 II 21

Sanctus Zenon (unbestimmt)

Jacobus 1412 I 1

Santperg

Andreas 1428 II 5

Sartoris

Fridricus 1429 II 6, 1435 I 15, 1435 II 14; Johannes Poter de Fridwerkch 1429 I 15; Nicolaus Sartoris 1411 I 15; Nicolaus de Gora 1411 I 7; Petrus de Kirchhain 1437 I 11

Satelboger

Erhardus Satelboger 1414 II 5

Satler

Nicolaus de Kunigsberg 1441 I 6

Sauigerue

Bartholomeus 1423 I 23

Sawnsheim s. Seinsheim

Saxo

Mathias Bernicz 1416 I 25, 1417 I 9

Schäßburg (ungar. Segesvár, ehem. Kom.

Nagy-Küküllö, rumän. Sighişoara, Sie-

benbürgen, Rumänien) Segswar

Johannes 1406 II 13

Schachner

Johannes 1441 I 8

Schadschek Septemcastrensis s. Schelken

Schärding (GB, OÖ) Schårding

Georgius Schrekch 1441 I 5

Schaithaimer

Johannes 1439 I 1

Schallerman

Johannes de Westfalia (Westualia, Westualus) (de Suzato) 1411 I 16, 1412 I, 1416 II, 1421 II

Scharb

Conradus de Lawben 1412 II 20

Scharendorf

Gregorius de Stuckardia 1433 I 15

Schatschelkch s. Schelken

Schaunberg (oö. Grafengeschlecht)

Schawmberg

Georius 1415 II 9

Schawer

Leonardus (Lienhardus) 1405 II, 1406 II 
Schebler Johannes 1437 I 6

Schedlein

Conradus 1416 II 20

Scheibbs (GB, NÖ) Scheibs, Scheybs Heinricus 1421 II 18, 1429 II 27

Schelken (ungar. Zselyk, ehem. Kom. Beszterce-Naszód, rumän. Jeica, Siebenbürgen, Rumänien) Schadschek Septemcastrensis, Schatschelkch Georius 1430 II 19, 1433 II 12

Schenck Maior s. Großschenk

Schenkch

Conradus de Külsheim 1418 II 17

Fridericus de Lymburg 1411 II 8 s. Limpurg

Petrus de Weybstat 1414 II 8

Schenkchenberger Johannes 1412 I 15

Scherffemberg Egidius de Lůbschütz 1442 I 4 Scheyrn

Petrus Tegerpek 1413 I 27

Schierling (Kr. Regensburg-Land, RB Oberpfalz, Bayern, Dtld.) Schirling Jacobus Frieshaymer 1422 II 13

Schilher Johannes 1409 II 12

Schiltdorffer Johannes 1430 I 4

Schintau (ungar. Sempte, ehem. Kom. Nyitra, Ungarn, slowak. Šintava, Slowakei) Schnitauia, Schnittauia, Sintauia Anthonius 1430 I 7, 1433 I 13, 1435 I 14, 1435 II 18

Schirling s. Schierling Schlesien (poln. Ślask, tschech. Slezsko, Gebiet beiderseits der oberen und mittleren Oder) Slesia, Schlak

Dominicus Hermanni 1437 II 4; Heinricus Prepticz 1427 I 6; Ladislaus 1427 II 5; Leutherus 1427 I 5

Schliersee (Kr. Miesbach, RB Oberbayern, Bayern, Dtld.) Sliersee Vlricus Prannt 1440 I 1
Schlupff

Richardus 1409 I 2

Schnitauia, Schnittauia s. Schintau

Schober

Chonradus de Hasfordia 1416 II 34

Scho̊naw

Andreas 1424 I 2

Scho̊nberg, Scho̊nperger

Nicolaus (de Olmüncz) 1428 I 7, 1431 II 21

Schöntrit

Leonardus de Salczburga 1438 II 2

Schơrling

Waltherus 1412 I 18

Scholym

Johannes 1415 I 11

Scholmar (ungar. Solymár, Kom. PestPillis-Solt-Kiskun, Ungarn) Smolna Philippus (Dino) 1412 I 2, 1417 II 5

Schram (unbestimmt)

Johannes 1437 II 10

Schrat

Mathias 1430 I 21

Schrekch

Georgius de Schårding 1441 I 5

Schrick (Gem. Gaweinstal, GB Mistelbach, $N O ̈)$

Michel 1424 I 6

Schürg

Conradus de Tranbach 1413 I 23

Schussenried s. Bad Schussenried

Schwabach (kreisfr. Stadt, RB Mittelfranken, Bayern, Dtld.) Swabach Johannes Resch 1428 II 1, 1433 II 10

Schwäbisch Gmünds. Gmünd

Schwäbisch Hall (Kr., RB Stuttgart, Baden-Württemberg, Dtld.) Halla

Conradus Eberhardi 1410 I 10; Conradus Velberger 1436 I 2; Johannes Felber 1413 I 9; Johannes Grůen 1441 I 9; Johannes Hofrichter 1424 I 12

Schwarzach b. Schwabach (kreisfr. Stadt, RB Mittelfranken, Bayern, Dtld.)

Swarczach

Krafto 1412 II 3, 1415 I 14 
Schweinfurt (kreisfr. Stadt, RB Unterfranken, Bayern, Dtld.) Sweinfordia Johannes 1419 I 18; Vlricus Horant 1439 I 6,1439 I 12

Screyber

Johannes de Weyssenburga 1428 I 9

Scriptoris

Heinricus de Frankfordia 1418 II 8; Johannes de Frankfordia 1413 II 1

Sculteti

Petrus de Russia 1423 I 6; Vrbanus de Jawr 1438 I 3, 1442 I 14

Seckau (GB Knittelfeld, Stmk.) Seccouia Johannes 1415 I 3

Seczel

Conradus de Rütlinga 1416 II 25

Seglauer

Conradus 1402 I 3; 1413 II

Segswar s. Schäßburg

Seybolt

Conradus de Memmingen 1418 I 23

Seydenliep

Conradus de Erfordia 1430 I 2

Seydlicz (unbestimmt, $H$ )

Nicolaus 1411 II 5

Seinreich

Otto 1411 II 14

Seinsheim (fränk. Reichsrittergeschlecht)

Sawnsheim

Erkengerus 1418 I 10

Seld, Sald, Selde

Johannes (de Wienna) 1406 II 18, 1419 II 22, 1420 II 23, 1425 I, 1429 I, 1437 I, 1439 II

Seler

Jodocus 1437 II 13, 1440 II 17

Selgenstad s. Seligenstadt

Selgerêt

Leonhardus de Mỏtnicz 1414 II 4

Seligenstadt (Kr. Offenbach, RB Darmstadt, Hessen, Dtld.) Selgenstad Petrus Amsteg 1415 II 4

Semlin (ungar. Zimony, ehem. Kom. Szerém, serb. Zemun, heute Stadtteil von Belgrad, Serbien) Semelin Johannes de Vngaria 1422 II 14
Sensensmid

Johannes 1438 II 13

Septem castra s. Siebenbürgen

Sepusiensis s. Zips

Serben (ungar. Cserbia, ehem. Kom.

Hunyad, rumän. Cerbia, Rumänien)

Zerblin

Andreas Michaelis 1421 I 23

Sesselman

Bertoldus 1415 I 22; Petrus 1415 I

21

Sewberleich

Wenczeslaus de Deschna 1416 II 13

Sews

Johannes 1434 II 9

Sybart

Thomas de Rückpass 1437 II 9

Sybmer

Conradus 1435 I 7

Siebenbürgen (ungar. Erdély, rumän. Transilvania, Ardeal, Rumänien) Septem

castra, Transiluana, Transsiluana,

Transiluanensis, Transsiluanensis,

Transsiluaniensis

Andreas 1430 II 20 (?), 1433 II 7;

Anthonius 1432 II 9, 1436 II 9, 1436 II 12; Antonius plebanus in Múllenbach 1422 I 21, 1425 II 4, 1427 II 12, 1428 I 11; Christanus 1409 II 4, 1436 II 17; Cristannus 1430 II 15, 1433 II 8, 1436 I 7, 1436 II 13; Demetrius Czuporis 1429 I 6; Dyonisius Jax 1414 I 24; Valentinus de Mulembach 1437 II 11; Georgius 1437 II 7; Georgius de Corona 1433 II 18, 1436 II 16, 1440 I 11; Johannes 1427 II 2; Ladislaus de Strig (Strigh) 1426 I 8, 1428 I 14, 1431 II 17, 1436 II 14; Laurencius de Corona 1429 I 10, 1430 II 32; Martinus 1428 II 28, 1430 I 32; Michael de Boden 1430 II 5; Michael Ekhart de Ardisch 1434 I 10; Michael (Heylsmar, Hylsmar) de Stolczenberg 1418 II 16, 1422 I 29, 
1435 I 12, 1435 II 12, 1435 II 16; Michael 1437 II 6; Michael de Insula Christiana 1418 II 26, 1438 II 18; Nicolaus 1413 I 2;

Nicolaus Lepes 1434 II 2, 1437 II 17, 1439 II 6, 1439 II 8; Petrus 1434 I 4; Petrus de Alba Transsiluana 1420 II 14, 1423 I 28, 1425 II 6; Petrus Nabok (Nobag, Nowag) 1420 II 18, 1423 I 30, 1428 I 10, 1428 I 12, 1432 II; Sigismundus 140ß II 10, 1412 II 14; Thomas 1410 I 2; Thomas 1411 II 16, 1413 I 29

Sigel

Johannes de Walsee 1412 II 4

Sigersdorffer

Caspar 1424 II 3

Symigiensis s. Sümeg

Syndel, Sündel

Wenczeslaus (de Brunna) 1414 II 9, 1419 I 1

Sindelfingen (Kr. Böblingen, RB Stuttgart, Baden-Württemberg, Dtld.) Sindel-

fing, Sindolfing

Bernhardus 1417 I 7, Johannes 1430 II 12

Sindrami, Sinderami, Syndrami Johannes 1405 II 4, 1406 II, 1409 I, 1411 II, 1415 II, 1420 I; Paulus de Nissa 1420 II 17, 1424 II 11

Sintauia s. Schintau

Sindringen (Stadtteil von Forchtenberg, Kr. Hohenlohekreis, RB Stuttgart, BadenWürttemberg, Dtld.) Syndring

Heinricus Wlpis 1414 I 14

Sitauia, Sittauia s. Zwittau

Slesia s. Schlesien

Slewser Albertus 1429 I 11

Slick, Slikch

Henricus 1432 I 11, Matheus 1428 II 3, 1430 II 31

Sliersee s. Schliersee

Slitt

Lucas de Prün 1426 II 12

Sluskow s. Ztoczów
Smaichingen (unbestimmt)

Andreas de Altershofen 1413 I 7

Smolna s. Scholmar

Soest (Kr., RB Arnsberg,Nordrhein-Westfalen, Dtld.) Suzatum

Johannes de Westfalia (Westualus) (Schallermann) 1411 I 16, 1412 I, 1416 II, 1421 II

Soldin (ehem. RB Frankfurt/Oder, poln.

Myślibórz, Polen) Soldinum

Math[ia]s Stephani 1416 II 8

Sompol

Johannes de Thodia 1416 II 1

Spêt

Johannes de Papenheim 1412 II 11, 1420 I 18

Span

Wilhelmus de Weyssenhorn 1421 I 4 Speyer (kreisfr. Stadt, RB Rheinhessen-

Pfalz, Rheinland-Pfalz, Dtld.) Spira,

Spirensis

Heinricus Engelfrid 1422 I 12; Heinricus de Helmstat 1413 I 15; Reynhardus de Helmstat 1412 I 7

Speyser

Nicolaus 1409 I 9, 1422 I 4; Philippus 1408 I 4

Speng

Conradus de Chiczing 1437 I 12

Spira, Spirensis s. Speyer

Sponsberg

Johannes de Wratislauia 1425 II 2

Stadelmair

Johannes 1433 I 8

Staffel (mehrfach in Dtld.)

Theodricus 1416 II 14

Stayn s. Stein

Stain Carniole s. Stein in Krain

Stainger

Johannes de Monaco 1431 I 9

Stamhawser

Vlricus de Noua Ciuitate 1424 I 14

Stampen

Procopius de Wratislauia 1421 II 19 Stang

Petrus de Ersperg 1416 I 4 
Stange

Johannes de Gorlicz 1432 II 7

Stankonis

Michael de Wratislauia 1426 I 7

Stark

Johannes 1412 II 18

Stauffer

Johannes de Plossenstauffen 1438 II 17

Stawdhaymer

Johannes de O̊tting 1430 II 8

Stawthaimer

Fridericus de O̊tinga 1407 II 3

Stawthaymer

Heinricus 1409 I 6

Steger

Michael 1432 I 3, 1438 II 19; Wil-

helmus de Mittenbald 1429 II 26

Steyer s. Steyr

Stein (Süddtld. und Schweiz mehrfach)

Lapis

Johannes 1415 I 16

Stein in Krain (Bez. Stein, slowen. Kamnik, Slowenien) Stain Carniole

Leonhardus 1441 I 4

Stein (mehrfach in Österreich oder Stein, slowen. Kamnik, Slowenien) Stayn

Leonardus 1423 II 4

Stểninger

Georius 1431 II 8

Stephani

Laurencius de Corpona 1420 I 7; Math[ia]s de Soldino 1416 II 8

Steyr (GB, OÖ) Steyer, Stira, Styra

Sigismundus 1425 II 1; Stephanus

Phister 1419 II 14; Wolfgannus

Temler 1430 II 24

Stok, Stock, Stokch

Nicolaus de Maiori Glogouia (Glogouia Maiori) 1413 I 17, 1421 I 20, 1422 I 25, 1422 II

Stolzenburg (Stoltzenburg) (ungar. Sze-

lindek, ehem. Kom. Szeben, Ungarn, rumän. Slimnic, Siebenbürgen, Rumänien) Stolczemberg, Stolczenberg, Stolczemburg

Cristannus Ewart 1434 I 5; Michael Hylsmar (Heylsmar) 1418 II 16,
1422 I 29, 1435 I 12, 1435 II 12, 1435 II 16

Stoll

Heinricus de Hamelburg 1414 I 15

Strasgang

Cristoferus Lusning 1422 I 11

Straßburg (franz. Strasbourg, Hauptstadt des Dep. Bas-Rhin, Frankreich) Argen-

tina, Argentinensis

Heinricus de Hewen 1421 II 9;

Johannes Merswein 1417 II 4,

1418 II 4; Johannes Pluemenek-

cher (Plůmenegker) 1434 I 14, 1436 II 15, 1439 II 10; Petrus

Ettenhayn 1424 II 4

Straubing (kreisfr. Stadt, RB Niederbayern, Bayern, Dtld.) Strawbinga

Johannes Heresinger 1422 II 8

Strawss

Oswaldus de Ratispona 1419 I 5

Strẻlen s. Střelitz

Streifdorf s. Streufdorf

Streyperger

Johannes 1403 I 5

Streit

Johannes de Goltperg 1435 II 9, 1437 II 20

Streytperger

Heinricus 1422 I 6

Strelitz (entweder tschech. Střelice, $G B$

Brünn-Umgebung, Tschechien oder GB

Litovel, Mähren, Tschechien oder GB

Znaim, Mähren, Tschechien oder Gem.

Hradec, GB Staab, Böhmen, Tsche-

chien oder Strelno, ehem. RB Brom-

berg, poln. Strzelno, Polen) Streàlen

Nicolaus Andree 1414 II 14

Stremphel

Johannes de Weissemburga 1434 I 9

Strenberger

Allexius 1429 I 9

Streufdorf (entweder Stadt Gunzenhau-

sen, Kr. Weißenburg-Gunzenhausen,

RB Mittelfranken, Bayern, Dtld. oder

Gem. Straufhain, Kr. Hildburghausen,

Thüringen, Dtld.) Streifdorf

Vlricus Waltheri 1435 I 5 
Strig, Strigh (unbestimmt, $H$ )

Ladislaus 1426 I 8, 1428 I 14, 1431 II 17, 1436 II 14

Strigonia, Strigonium, Strigoniensis $s$. Gran

Stròmberger Johannes 1438 II 7

Strohofer Thomas 1437 I 5, 1439 II 5

Stubner, St'ubner, Stůbner

Nicolaus 1430 I 11, 1433 I 12, 1435 I 13, 1435 II 13

Stüblau (wohl ehem RB Danzig, Dtld., poln. Steblewo, Westpreußen, Polen) Stubelaw

Andreas Scho̊naw 1424 I 2

Stürpecher

Nicolaus de Glocz 1434 I 7, 1440 I 5, 1440 II 12

Stüxs

Johannes de Weyden 1438 I 6

Stuhlweißenburg (ung. Székesféhérvár, Ungarn) Alba Regalis

Petrus Pauli 1416 I 20

Stůbner s. Stubner

Stuttgart (kreisfr. Stadt, RB, Landeshauptstadt, Baden-Württemberg, Dtld.) Stukardia, Studkardia

Gregorius Scharendorf 1433 I 15; Johannes 1418 II 13; Johannes Gotz 1429 II 24

Sümeg (Kom. Zala, Ungarn) Symigiensis Paulus 1415 I 1

Sundel $s$. Syndel

Sulz (entweder Stadtteil von Lahr (Schwarzwald), Kr. Ortenaukreis, RB Freiburg, Baden-Württemberg, Dtld. oder Sulz am Neckar, Kr. Rottweil, RB Freiburg, Baden-Württemberg, Dtld. oder Gem. Bad Rippoldsau-Schapbach, Kr. Freudenstadt, RB Karlsruhe,
Baden-Württemberg, Dtld. oder Sulz am Eck, Stadt Wildberg Kr. Calw, Kr. Calw, RB Karlsruhe, Baden-Württemberg, Dtld. oder Stadt Rothenburg ob der Tauber, Kr. Ansbach (Land), RB Mittelfranken, Bayern, Dtld.) Sulcz Johannes Swerger 1413 I 28

Sulzbach (Süddtld. mehrfach) Sulczpach, Salczbach

Johannes Pềrtl (Bẻrtel) 1438 II 5, 1441 I 11; Petrus 1426 I 1; 1428 I 15

Sunperger

Vlricus de Orengew 1430 II 3, 1434 II 10, 1438 I 15

Superior Lapis s. Oberstein

Sutoris

Johannes de Freynstat 1437 II 2

Suzatum s. Soest

Swabach s. Schwabach

Swarat

[Nicolaus] 1420 I 21, Philippus (de Brixina) 1433 I 7, 1436 II 18, 1440 II 13

Swarczach s. Schwarzach b. Schwabach

Sweydnitz

Johannes 1410 I 13

Sweygkers s. Schweiggers

Leonardus Gruber 1420 I 4

Sweinbart s. Groß-Schweinbart

Sweinfordia s. Schweinfurt

Swentenkrieg

Georgius de Welss 1435 II 7

Swerger

Johannes de Sulcz 1413 I 28

Swolla (tschech. Zvole, GB Bystrice nad

Pernštejnem, Mähren, Tschechien)

Swola

Bohussius 1435 II 2, 1440 II 15

Szent Laszló (Ungarn mehrfach) Villa Las

Nicolaus Atyser 1415 II 3
Tabernatoris

Johannes 1416 I 26
Tagersheim, Tagersheym, Tagershaim, Tagerhaim s. Dagersheim 
Tamsweg (GB, Sbg.) Tềmsweg

Wolfgangus 1441 II 2

Tandorfer, Tandorffer

Heinricus (de Nuremberga) 1413 II 9, 1417 II 1, 1418 II 5, 1419 I 17

Tapolca (Kom.Zala, Ungarn) Tapolcza,

Tapoltza

Johannes 1434 I 1, 1436 I 5,1438 I

12, 1438 I 17

Tarbatensis s. Dorpat

Tardoskedd (ehem. Kom. Nyitra, Ungarn, slowak. Turdošovce, Slowakei) Tardaschedy Tardascheydn Tardeschedy Fabianus 1420 I 11, 1421 I 18, 1424 I 21, 1424 II 13

Tartlau (ung. Prázsmár, rumän. Prejmer,

Bez. Kronstadt) Tartala

Nicolaus 1413 I 2

Tebershaymer

Bertoldus 1425 I 5

Tegernsee (Kr. Miesbach, RB Oberbayern,

Bayern, Dtld.)

Vlricus Kaltemprunner 1441 I 2

Tegerpek

Petrus de Scheyrn 1413 I 27

Teisser

Erasmus 1436 II 6

Telkener

Johannes de Cremniczia 1432 II 5

Temeswar (ung. Temesvár, rumän.

Timişoara, Rumänien) Temeswar,

Temswar, Themezwar

Nicolaus 1418 I 8, 1421 I 17, 1422 I 26

Temler

Wolfgannus de Stira 1430 II 24

Tenniger

Leonhardus de Monaco 1429 II 22

Teschner

Petrus de Wratislauia 1411 I 13

Tewfel

Maternus de Brunna 1440 II 2

Thodia (unbestimmt)

Johannes Sompol 1416 II 1

Thorn (poln. Torún, Bez. Bromberg, Westpreußen, Polen) Toran
Johannes Fyol 1418 I 15; Petrus 1420 I 13

Tifer

Fredericus de Constancia 1420 II 2

Tirna (Wiener Ritterbürgerfamilie) Tyrna Achacius 1406 II 4

Tyssawassan, Tysswassan s. Varsány

Tittmoning (Kr. Traunstein, RB Oberbayern, Bayern, Dtld.) Tytmaning

Johannes Rèwtemberger 1420 II 1

Tyzzawazzan s. Varsány

Tywing s. Tübingen

Toblach (italien. Dobbiaco, Südtirol, Italien) Tullacum

Cristofferus Arnolt 1429 II 12; Johannes Arnolt 1429 II 11

Toledo (Hauptstadt der Prov. Toledo, Spanien) Toletanus, Toletanensis

Alfonsus Gamezii (Gomecii, Gomezii) 1424 II 5, 1428 II 14,1430 I 23, 1430 I 24

Toliatoris

Johannes de Crossna 2 gr. 1429 I 3, 1430 II 26

Tols

Jacobus de Rudlinga 1429 II 2

Toran s. Thorn

Tornaw

Johannes de Lutko 1411 II 10, 1420 II 26

Traboldi

Johannes de Bruxella 1411 II 3

Traföß (Gem. Pernegg, GB Bruck an der Mur, Stmk.) Trafess

Johannes Preller 1423 II 2

Traiectum, Traioctensis s. Maastricht

Tranbach s. Kronach

Transiluana, Transsiluana, Transiluanensis, Transsiluanensis, Transsiluaniensis

s. Siebenbürgen

von Traun (österr. Herrengeschlecht) Trawner

Conradus 1406 II 1

Trier (kreisfr. Stadt, RB, Rheinland-Pfalz, Dtld.) Treverensis, Treuerensis

Adolffus de Eppenstain 1424 I 19; Johannes de Manderscheyt 1417 I 1; Phylippus de Cronemberg 1414 I 2 
Troppau (tschech. Opava, Schlesien, Tschechien) Opauia Johannes Rötlini 1428 I 5

Troppawer

Paulus de Wienna 1419 II 7

Trudelonis

Bruno de Ytstein 1417 I 15

Tübingen (Kr. und RB, Baden Württem-

berg, Dtld.) Tywing

Conradus Herbeyn 1426 I 5
Tưrndl

Johannes 1407 II 5; Theobaldus 1438 II 10

Tullacum s. Toblach

Tullinger

Johannes 1418 II 12

Tulln (GB, NÖ) Tulna

Thomas 1434 II 6

Turinge

Johannes de Prawnsperg 1413 I 24

Vbelman

Johannes Vbelman 1416 I 3

Überlingen (Kr. Bodenseekreis, RB Tü-

bingen, Baden-Württemberg, Dtld.)

Vberling

Jodocus Rot 1420 I 5, 1431 II 20

Ulm (Kreisfr. Stadt, RB Tübingen, Baden-

Württemberg, Dtld.) Vlma

Kaspar Herr 1411 II 11; Conradus 1427 II 8, 1431 I 15, Jacobus Angeli 1417 II 12; Johannes Hofleich 1427 II 9, 1432 I 19; Johannes Wurcher 1421 II 15;

Wilhelmus Kraft 1440 I 2

Ungarn Ungaria, Vngaria

Franciscus Johannis de Luko 1425 I

3; Georius de Egerzeg 1424 I 10, 1427 II 14, 1430 I 31 (?), 1430 II 36; Georius Petri de Briswicz 1425 I 2, Johannes de Semelin

1422 II 14; Nicolaus de Waradino 1414 I 7, 1417 II 2, 1419 II 23, 1421 I 19

Vngarus

Georius de Egersek (Egerzeg) 1424 I

10, 1427 II 14, 1430 I 31?, 1430 II 36

Unterstockstall (Gem. Kirchberg am Wagram, GB Kirchberg am Wagram, NÖ) Inferior Stokchstal Jacobus 1416 I 15

Uttendorf (Gem. Prinzendorf, GB Neulengbach, NÖ oder OÖ mehrfach oder GB Mittersill, Sbg. oder Markt Gangkofen, Kr. Rottal-Inn, RB Niederbayern, Dtld. oder Gem. Haselbach Kr. Straubing-Bogen, Kr. Straubing-Bogen, RB Niederbayern, Bayern, Dtld.) Thomas de Vttendorf 1427 I 8, 1431 I 11

- V siehe F -

$-\mathrm{W}-$

Wacia, Waciensis s. Waitzen

Wager

Siluester Wager de Patauia 2 gr. 1416 I 14

Waiblingen (wohl Kr. Rems-Murr-Kreis,

RB Stuttgart, Baden-Württemberg,

Dtld.) Waibling
Heinricus Eglinger de Waibling 2 gr. 1417 I 14

Waitzen (ungar. Vác, Kom. Pest-Pilis-Solt-

Kiskun, Ungarn) Wacia, Waciensis,

Bacia, Baciensis

Emericus 1430 II 13; Georius 1431 II 7; Georgius 1440 I 6; 
Heinricus Rosemberg 1416 II 28; Simon 1430 II 6

Walczhuett s. Waldshut-Tiengen

Waldarn (unbestimmt)

Michel Liebhardi 1419 I 23

Waldbrunn Kr. Würzburg (Kr. Würzburg

Land), RB Unterfranken, Bayern,

Dtld.)

Johannes Woluisheim 1412 II 12

Waldeck oder Waldegg (mehrfach in Dtld.)

Waldegk

canonicus et custos ecclesie Frisingensis 1416 I 2

Waldhausen (GB Perg, OÖ) Walthausen,

Walthawsen, Walthusen

Martinus 1419 I 12, 1425 II 8, 1428 II 13, 1430 II 34, 1438 II, 1440 I, 1442 I

Stephanus 1427 I 9

Waldhütten (ungar. Váldhid,ehem. Kom.

Nagy Küküllö, Ungarn, rumän.Val-

chid, Siebenbürgen, Rumänien) Walthuden

Nicolaus 1406 II 11

Waldshut-Tiengen (Kr. Waldshut, RB

Freiburg i. Br., Baden-Württemberg,

Dtld.) Walczhuett

Bertoldus Ekkinger 2 gr. 1438 II 6

Walich

Sigismundus de Aybling 1426 II 17

Waller

Vincencius 1416 I 9

Walsee s. Bad Waldsee

Walthausen, Walthawsen $s$. Waldhausen

Waltheri

Vlricus de Streifdorf 1435 I 5

Walthusen s. Waldhausen

Wanislant

Martinus de Rosenheim 1416 II 17

Wanow (tschech. Vaňov, GB Aussig, Böh-

men, Tschechien) Bano

Bohoslaus 1423 II 1, 1426 II 21

Waradinum, Waradiensis s. Großwardein

Warmiensis s. Ermland

Warnaw s. Bernau bei Berlin

Warnhofer

Fridericus de Astauia 1413 I 8
Wartenberg (tschech. Stráž pod Ralskem, GB Niemes, Böhmen, Tschechien oder Ortsteil von Budikovice, GB Náměst' nad Oslavou, Mähren, Tschechien)

Wartemberg

Conradus Wolff 1419 II 5, 1422 I 10; Johannes Wolf 1411 I 6; NicolausWolff 1411 I 5; Nicolaus Wolf 1419 II 4, 1422 I 9

Wasserburg (wohl Wasserburg a. Inn, Kr. Rosenheim (Land), RB Oberbayern, Bayern, Dtld. oder Wasserburg (Bodensee), Kr. Lindau (Bodensee), RB Schwaben, Bayern, Dtld.)

Cristannus 1424 I 18

Weybstat (unbestimmt)

Petrus Schenkch 1414 II 8

Weiden (Bayern, Baden-Württemberg, Rheinland-Pfalz, Hessen mehrfach)

Weyden

Emeramus Parkstainer 1439 I 9; Johannes Stůxs 1438 I 6

Weidenbach (ungar. Vidombák, ehem. Kom. Brassó, Ungarn, rumän. Ghimbav, Siebenbürgen, Rumänien) Weydenpach, Weydembach Petrus 1433 II 17; Tylmannus Helwich 1421 II 14

Weiding (wohl Weiding Kr. Cham, Kr. Cham, RB Oberpfalz, Bayern, Dtld. oder Weiding Kr.Schwandorf, Kr. Schwandorf, RB Oberpfalz, Bayern, Dtld. oder Weiding mehrfach in Bayern, Dtld. oder Weidingen, Kr. Bittburg-Prüm, RB Trier, Rheinland-Pfalz, Dtld.) Weyding

Nicolaus Mayr 1421 I 12

Weil (mehrfach in Süddeutschland; oder Wil, Schweiz, Kanton St. Gallen oder

Kanton Zürich) Byla, Wyla, Wil

Albertus 1408 I 6; Heinricus 1436 II 7

Weinsberg (Kr. Heilbronn (Land), RB

Stuttgart, Baden-Württemberg, Dtld.)

Wymbergensis

Conradus 1420 I 15

Weinschenk

Nicolaus de Rudlinga 1418 II 2 
Weinstok

Vincencius de Holenburg 1409 II 8

Weißenburg i. Bay. (Kr. Weißenburg-Gunzenhausen, RB Mittelfranken, Bayern, Dtld. oder Weißenburg, Unterelsaß, franz. Wissembourg, Dep. Bas-Rhin,

Frankreich) Weissemburga, Weyssenburga, Weyssemburga

Jacobus Luf 1422 II 7; Jacobus Rosenczwey 1442 I 11; Johannes Screyber1428 I 9;

Johannes Stremphel 1434 I 9; Vlricus (Czand) 1415 II 7, 1418 II 7, 1427 II 10, 1428 I

Weißenhorn (Kr. Neu-Ulm, RB Schwaben, Bayern, Dtld.) Weyssenhorn

Wilhelmus Span 1421 I 4

Weissenkirchen (mehrfach in Ö)

Wolfgangus 1412 II 16

Weytensdorffer

Gregorius 1434 II 7

Weitra (GB Gmünd in Niederösterreich,

$N O ̈)$ Weytra

Jacobus 1413 I 20

Weiz (GB, Stmk.) Weytz

Johannes Hymel 1411 I 8

Welczer

Cristofferus 1420 I 9

Wels (GB, OÖ) Welss, Welsa

Conradus Pruglegker 1440 II 8; Georgius Swentenkrieg 1435 II 7;

Wenke Martinus Hublinger 1415 II 14

Nicolaus 1422 II 2, 1423 II 9

Werder

Vlricus de Papenheim 1413 II 16

Werthart

Vlricus de Ratispona 1421 II 2

Wesprimiensis s. Veszprim

Wessely (mehrfach in Böhmen und Mäh-

ren, Tschechien) Wessele

Wenczeslaus 1424 I 1

Westerndorffer

Caspar de Lantzhůt 1436 I 6

Westersteter

Rudolfus 1412 I 19
Westfalen, Dtld. Westfalia, Westualia,

Westualus

Johannes (Schallermann) (de Suzato)

(Westualus) 1411 I 16, 1412 I, 1416 II, 1421 II

Wichtorff

Hinricus 1403 I 4

Wyel

Nicolaus de Wlessna 1418 I 1

Wien (österr. Bundesland und Bun-

deshauptstadt) Wienna, Wynna,

Wiennensis, Wyenna, Wýnna

Conradus Bladeck (Bladek) 1420 I

3, 1424 II 10, 1426 I 10, 1427 I;

Johannes Folczian 1414 II 1;

Johannes Hoffkircher 1426 I

4; Johannes Seld (Saeld, Selde)

1406 II 18; 1419 II 22, 1420 II

23, 1425 I, 1429 I, 1437 I,

1439 II; Nicolaus Ysenhủtel

1413 II 11; Paulus (Påwrl)

1409 II 11, 1419 II 19, 1419 II

20, 1420 II 22, 1420 II, 1425 II,

1428 II, 1431 II; Paulus Troppa-

wer 1419 II 7, Petrus Dekinger

1412 II

- Sankt Stephan

Gerhardus Vischpekch 1411 I;

1415 I; Petrus Dekchinger

1418 II; Vdalricus de Haydreichstain 1431 II 1

Wieselburg (GB Scheibbs, NÖ) Wiselburg

Andreas de Falkenberg 1413 I 5

Wiesensteig (Kr. Göppingen, RB Stuttgart,

Baden-Württemberg, Dtld.) Wisen-

staig, Wysenstaig

Petrus Naffczer (Nafczer) 1438 II 4, 1440 II 5, 1441 I 12

Wil, Wyla $s$. Weil

Wilhelmi

Johannes de Corona 1424 II 2

Wymbergensis s. Weinsberg

Wingler

Johannes de Medling 1432 II 6

Winterburg

Johannes 1413 II 4 
Winz (ung. Alvinc, rumän. Vințu de Jos, Bez. Hunedoara, Rumänien) Wincz Johannes 1431 II 12

Winzig (poln. Wińsko, Stadt nördl. von

Wohlau, Niederschlesien, Polen) Winczig

Johannes Cristoferi 1413 II 15

Wirgil s. Wörgl

Wirnt

Johannes 1406 II 16

Wyrsing

Stephanus 1406 II 8

Wirsung

Chunradus de Lantzhuta 1411 I 10

Wirthelm s. Birthälm

Wyschehrad in Prag (tschech. Vyšsehrad, GB

Vnitřni Praha, Böhmen, Tschechien)

Wissegradensis

Andreas Nas 1412 II 13; Petrus de

Sulczpach 1426 I 1, 1428 I 15

Wiselburg s. Wieselburg

Wismar Kr. Wismar (kreisfr. Stadt,

Mecklenburg-Vorpommern, Dtld.)

Wismaria

Heinricus Wollemberg 1418 II 18

Wismayr

Hainricus 1433 I 11

Wispeck

Georius 1418 II 10

Wissegradensis s. Wyschehrad

Wittenberg (Kr., Sachsen-Anhalt, Dtld.)

Wittemberg

Andreas de Wittemberg 2 gr. 1416 II 22; 1421 I 22

Wlínow (tschech.Vlčnov, GB Chrudim,

Böhmen, Tschechien oder Wlčnow,

tschech. Vlčnow, GB Planitz, Böhmen,

Tschechien) Wlocznow

Wenczeslaus de Wessele 1424 I 1

Wlessna (unbestimmt)

Nicolaus Wyel 1418 I 1

Wlpis

Heinricus de Syndring 1414 I 14

Wólflein

Johannes 1410 I 9

Wörgl (GB Kufstein, T) Wirgil

Leonhardus Kellner 1435 I 4
Wogawer

Johannes 1408 I 8

Woiczdorff

Franciscus 1429 I 2, 1430 I 29, 1434 II 14,1434 II 15

Wolf (Wolff)

Conradus 1419 II 5, 1422 I 10; Johannes 1411 I 6; Nicolaus 1419 II 4, 1422 I 9 (Bruder des Conradus); Nicolaus 1411 I 5

Wolfach (Kr. Ortenaukreis, RB Freiburg i. Br., Baden-Württemberg, Dtld.)

Erhardus 1437 I 13, 1441 II 7

Wolfpach s. Wolfsbach

Wolfsbach (entweder GB St. Peter in $\operatorname{der} A u, N O ̈$ oder slowen. Vučja Ves, ehem. Gem. Uggowitz (slowen. Ukve), heute Valbruna, Gem. Tarvis, italien.

Commune di Tarvisio, Italien oder Wolfsbach, auch Wolfsbüchel, slowen. Volčji Potok, Gem. Stein, slowen.

Kamnik oder Gem. Domschale, slowen. Domžale, Bez. Stein, Krain) Wolfpach Martinus 1422 I 14

Wolfsberg (Ktn. oder Stmk. mehrfach)

Wolffsperg

Caspar 1427 II 15

Wolfsheim (Kr. Mainz-Bingen, RB Rheinhessen-Pfalz, Rheinland-Pfalz, Dtld.)

Wülfesheim

Johannes Raczenhawsen 1421 I 14

Wolkersdorf (GB, NÖ)

Martinus 1435 II 3

Wollemberg

Heinricus de Wismaria 1418 II 18

Woluisheim

Johannes de Waldbrunn 1412 II 12

Worach s. Póráz

Woslawicz s. Poslowitz

Wratislauia, Wratislauiensis s. Breslau

Würzburg (kreisfr. Stadt, RB Unterfran-

ken, Bayern, Dtld.) Herbipolis, Her-

bipolensis

Demetrius de Gich 1414 II 2; Erkengerus de Sawnsheim 1418 I 10; Fridericus Schenkch de Lymburg 1411 II 8; Gregorius Ledenter 
1412 II 5; Theodorus de Gich 1415 I 5

Wücherl

Johannes de Lewben 1418 II 22

Wulfesheim s. Wolfsheim

Wurcher

Wurrm

Johannes de Vlma 1421 II 15
$-\mathrm{C}, \mathrm{Cz}, \mathrm{Z}-$

Zagrabia, Zagrabiensis s. Agram

Zákány (Kom. Somogy, Ungarn) Zakan

Thomas 1415 I 4

Zammelsberg (Mgm. Weitensfeld im Gurk-

tal, GB St. Veit an der Glan, Ktn., Ö)

Zumelsperg, Zůmelsperg

Georius plebanus in Grifen 1414 I 9, Hertwicus 1421 I 2

Czand

Vlricus de Weyssemburga 1415 II 7 , 1418 II 7, 1427 II 10, 1428 I

Zánka (viell. Kom. Zala, Ungarn) Campa

Heinricus 1430 I 22

Czap

Johannesde Bechthem 1429 I 12; Vlricus de Bechthem 1429 I 13

Zedlicz

Nicolaus canonicus ecclesie Bregensis 1418 I 17

Czedlicz

Bartholomeus de Hiersberg 1423 II 7

Zegenberg s. Ziegenberg

Zeydlaraer

Chonradus de Monaco 1410 II 2

Zelesna (Železna, tschech. Železná, polit.

Bez. Kladno, GB Unhost, Böhmen,

Tschechien)

Stephanus 1418 II 27

Czeller

Johannes 1432 II 3; Martinus de

Grewàtzensteten 1423 I 12, 1431 I 14

Czener

Caspar de Patauia 1431 I 6, 1434 II 11
Cristannus de Salina 1412 I 20;

Wolfgangus de Freynstat 1414 I

6, 1437 II 15

Wulco

Hermannus de Frankenfordis 1417 II 9

Wurcia s. Burzenland

Wusla s. Bušince
Zepeck, Czepeck

Johannes de Laybaco (plebanus ad Sanctum Vitum prope Laybacum) 1413 I 13, 1431 II 25

Cepus, Czepusiensis s. Zips

Zerblin s. Serben

Czerod s. Csejd

Cibiniensis, Cibinium s. Hermannstadt

Ziegenberg (in Ostpreußen und Pommern mehrfach) Zegenberg

Pilgrimus 1413 I 26, 1418 II 6

Zyglawer s. Seglauer

Cziller

Petrus 1429 I 14

Zimischem

Johannes 1413 I 25

Czink, Zink

Johannes 1413 II 13; Nicolaus 1433 I 1

Zips (slowak. Spiš, früher ung. Kom. Szepes, jetzt Slowakei) Cepus, Sepusiensis, Czepusiensis

Caspar de Brezewicz 1426 II 11;

Georius 1412 I 21; Johannes

1439 I 3; Johannes de Pudlino 1427 I 1, 1442 I 13

Zirklach (ehem. Land Krain, Bez. Krainburg, slowen. Cerklje, Slowenien)

Cirkla Jacobus Rayner 1434 I 2

Zirnperger

Petrus 1423 II 6

Cisterdarffer

Sigismundus 1430 II 7 
Zistersdorf (GB, NÖ) Czisterstorf Wolfgangus Payer (Payr) 1421 I 9, 1430 I 26

Zittau (Kr. Löbau-Zittau, RB Dresden, Sachsen, Dtld.) Zitauia

Johannes Finsterwald(er) 1416 II 6 Ztoczów (Galizien, Polen) Sluskow Jeroslaus 1426 II 1

Znaim (tschech. Znojmo, polit. Bez. und GB, Mähren, Tschechien) Znoyma Johannes 1417 I 13

Czopot

Johannes1429 I 5, 1431 II 22
Zsana (Kom. Pest, Ungarn) Sana Paulus 1414 I 16, 1417 II 3

Czschach s. Császár

Zumelsperg, Zůmelsperg s. Zammelsberg Czuporis

Demetrius 1429 I 6

\section{Czwinger}

Johannes de Basilea. 1424 I 13

Zwittau (tschech. Svitavy, GB, Mähren,

Tschechien) Sitauia, Sittauia, Zwitauia

Andreas 1417 I 5; Johannes

(Finsterwald[er]) 1416 II 6, 1420 II 25, 1422 I 27; Wenczeslaus 1422 I 8 



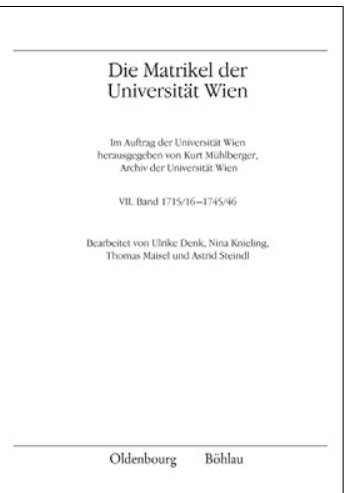

BEARBEITET VON ULRIKE DENK,

NINA KNIELING, THOMAS MAISEL

UND ASTRID STEINDL

IM AUFTRAG DER UNIVERSITÄT WIEN

HERAUSGEGEBEN VON KURT MÜHLBERGER,

ARCHIV DER UNIVERSITÄT WIEN

DIE MATRIKEL DER

UNIVERSITÄT WIEN

VII. BAND 1715/16-1745/46

VI. REIHE: QUELLEN ZUR GESCHICHTE DER

UNIVERSITÄT WIEN, 1. ABTEILUNG

Der vorliegende siebente Editionsband setzt die Reihe der Wiener Hauptmatrikeledition fort und erschließt 31 Studienjahre, nämlich den Zeitabschnitt $1715 / 16$ bis $1745 / 46$. Er enthält Eintragungen über insgesamt 6.764 Universitätsbesucher mit Herkunfts- und Standesangaben sowie jährlich die Ergebnisse der Rektorswahl. Matrikel- und Aktenteil werden durch die Liste der Rektoren sowie durch ein Namen- und ein Ortsregister erschlossen.

(PUBLIKATIONEN DES INSTITUTS FÜR ÖSTERREICHISCHE GESCHICHTSFORSCHUNG, VII. BAND, I. ABTEILUNG)

2011. XLIV, 500 S. BR. $203 \times 282$ MM.

ISBN 978-3-205-78655-9 [A]

ISBN 978-3-486-70459-4 [D]

BÖHLAU VERLAG, WIESINGERSTRASSE I, IOIO WIEN. T: + 43 (o) I 33024 27-O BOEHLAU@BOEHLAU.AT, WWW.BOEHLAU-VERLAG.COM | WIEN KÖLN WEIMAR 


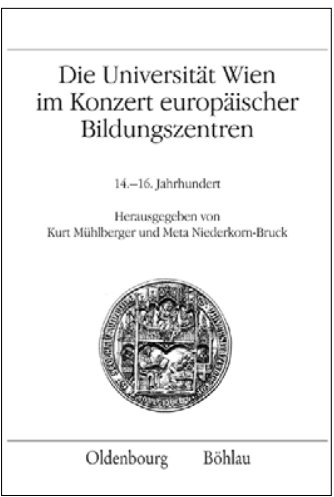

HG. VON KURT MÜHLBERGER UND

META NIEDERKORN-BRUCK

DIE UNIVERSITÄT WIEN IM

KONZERT EUROPÄISCHER

BILDUNGSZENTREN

14.-16. JAHRHUNDERT

VERÖFFENTLICHUNGEN DES INSTITUTS FÜR

ÖSTERREICHISCHE GESCHICHTSFORSCHUNG

BAND 56

Die von Herzog Rudolf IV. am 12. März 1365 gegründete Alma Mater Rudolphina hat das „Konzert europäischer Universitäten“ um eine wesentliche Stimme erweitert. Nach der Prager Karlsuniversität (1347/48), dem ersten beständigen Generalstudium nördlich der Alpen, hatte eine Gründungswelle eingesetzt, die die Bildungslandschaft des Reiches und darüber hinaus entscheidend prägte. Durch die Mobilität von Lehrenden und Lernenden war die Einbindung in das internationale Netzwerk des Wissens gewährleistet. Im Jahre 2007 wurde der Wiener Arbeitskreis für Universitätsgeschichte als eine Kooperation des Instituts für Österreichische Geschichtsforschung und des Archivs der Universität Wien ins Leben gerufen und dazu international renommierte Historikerinnen und Historiker eingeladen. Es wurde versucht, den Standort des Wiener Studiums im großen „europäischen Orchester der Wissenschaft“ zu bestimmen.

Beiträge von Harald Berger, Peter Csendes, Ulrike Denk, Christine Glassner, Helmuth Grössing, Gernot Heiss, Christian Hesse, Thomas Kühtreiber, Christian Lackner, Kurt Mühlberger, Meta Niederkorn-Bruck, Johannes Seidl, Karl Ubl, Marija Wakounig

2010. 278 S. BR. 26 S/W- U. 3 FARB. ABB. $170 \times 240$ MM.

ISBN 978-3-205-78490-6 [A], 978-3-486-59224-5 [D]

BÖHLAU VERLAG, WIESINGERSTRASSE I, IOIO WIEN. T : + 43(o) I 33024 27-O BOEHLAU@BOEHLAU.AT, WWW.BOEHLAU-VERLAG.AT | WIEN KÖLN WEIMAR 


\section{PUBLIKATIONEN}

\section{DES INSTITUTS FÜR ÖSTERREICHISCHE GESCHICHTSFORSCHUNG}

VI. Reihe: Quellen zur Geschichte der Universität Wien 3. Abteilung: Die Matrikel der Wiener Rechtswissenschaftlichen Fakultät

Band I

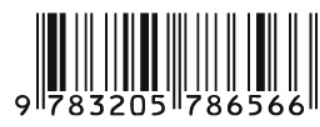

Böhlau Wien ISBN 978-3-205-78656-6

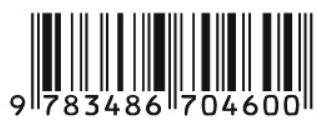

Oldenbourg München ISBN 978-3-486-70460-0 